\title{
Evaporation and dissolution of droplets in ternary systems
}

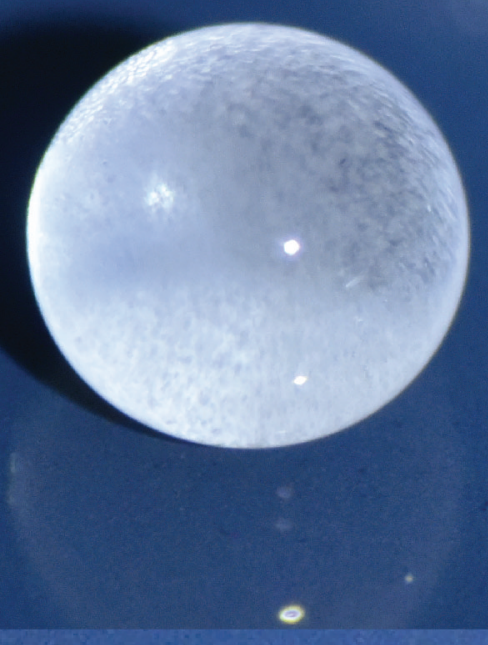




\section{EVAPORATION AND DISSOLUTION OF DROPLETS IN TERNARY SYSTEMS}

Huanshu Tan

(谭唤书) 


\section{Graduation Committee:}

Prof.dr.ir. J.W.M. Hilgenkamp (chairman) University of Twente

Prof.dr. D. Lohse (supervisor)

University of Twente

Prof.dr. X. Zhang (co-supervisor)

University of Alberta \& U. Twente

Prof.dr.ir. N.E. Benes

University of Twente

Prof.dr.ir. R.G.H. Lammertink

University of Twente

Dr. P.S. Clegg

Prof.dr.rer.nat. S. Hardt

University of Edinburgh

Technische Universität Darmstadt

The work in this thesis was carried out at the Physics of Fluids Group, MaxPlanck-Center Twente for complex fluid dynamics, Mesa+ Institute, and J. M. Burgers Centre for Fluid Dynamics, Faculty of Science and Technology, University of Twente. This thesis was financially supported by the China Scholarship Council and the Netherlands Organisation for Scientific Research.

Cover design: Huanshu Tan.

Publisher: Huanshu Tan,

Physics of Fluids Group, University of Twente,

P.O. Box 217, 7500 AE Enschede, The Netherlands

pof.tnw.utwente.nl

Copyright (C) Huanshu Tan, Enschede, The Netherlands 2018

All rights reserved. No part of this book may be reproduced, stored in a retrieval system, or transmitted in any form or by any means, without written permission of the author.

Print: Gilderprint, Enschede

ISBN: 978-90-365-4592-1

DOI: $10.3990 / 1.9789036545921$ 


\section{EVAPORATION AND DISSOLUTION OF DROPLETS IN TERNARY SYSTEMS}

DISSERTATION

to obtain

the degree of doctor at the University of Twente, on the authority of the rector magnificus,

Prof.dr. T.T.M. Palstra,

on account of the decision of the graduation committee to be publicly defended

on Friday the 24th of August 2018 at 16:45

by

Huanshu Tan

(谭唤书)

Born on $19^{\text {th }}$ of October 1987

in Jining, Shandong province, China 
This dissertation has been approved by the supervisors:

\author{
Prof. dr. Detlef Lohse \\ and
}

Prof. dr. Xuehua Zhang 


\section{Contents}

$\begin{array}{llr}1 & \text { Introduction } & 1\end{array}$

1.1 Motivations . . . . . . . . . . . . . . . . . . 1

1.2 Small-scale world of droplets $\ldots \ldots \ldots$. . . . . . . . 3

1.3 Evaporation dynamics of droplets . . . . . . . . . . . . . . 7

1.4 The "ouzo effect" and ternary phase diagram . . . . . . . . 9

1.5 A guide through this thesis $\ldots \ldots \ldots \ldots \ldots \ldots$

\begin{tabular}{lll}
\hline I Ouzo drops & 17
\end{tabular}

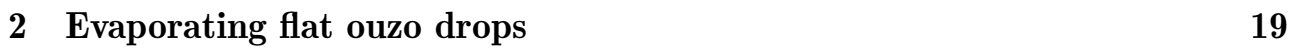

2.1 Introduction . . . . . . . . . . . . . . . . . . . . . . 19

2.2 Results and discussion $\ldots \ldots \ldots \ldots \ldots$

2.3 Methods . . . . . . . . . . . . . . . . . . . . . . . . . 29

2.4 Conclusions $\ldots \ldots \ldots \ldots \ldots$

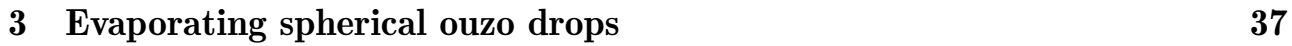

3.1 Introduction . . . . . . . . . . . . . . . . . . 38

3.2 Materials and methods $\ldots \ldots \ldots$. . . . . . . . . . 40

3.3 Experimental results . . . . . . . . . . . . . . . . . . . . . . . 42

3.4 FEM numerical modeling for evaporation $\ldots \ldots \ldots$. . . . . 47

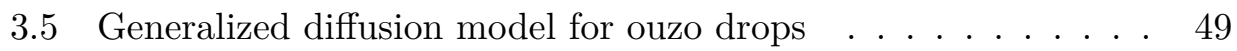

3.6 Conclusions . . . . . . . . . . . . . . . . . . . . 54

3.7 Supplementary material $\ldots \ldots \ldots \ldots \ldots \ldots$

\begin{tabular}{|lll}
\hline 4 & Dissolving ouzo drops & 57
\end{tabular}

4.1 Introduction . . . . . . . . . . . . . . . . . . . . 58

4.2 Experimental method $\ldots \ldots \ldots \ldots$

4.3 Dissolution process $\ldots \ldots \ldots \ldots$

4.4 Spontaneous emulsification $\ldots \ldots \ldots \ldots$

4.5 One-dimensional multicomponent diffusion model . . . . . . . . 69 
4.6 Model predictions . . . . . . . . . . . . . . . . . . 76

4.7 Discussion on the fluid dynamics of the system . . . . . . . . 82

4.8 Summary and conclusions . . . . . . . . . . . . . . 85

\begin{tabular}{|lll}
5 & Evaporating pure, binary \& ternary drops & 87
\end{tabular}

5.1 Introduction . . . . . . . . . . . . . . . 88

5.2 Experimental setup . . . . . . . . . . . . . . . . . . 90

5.3 Axisymmetric investigation $\ldots \ldots \ldots \ldots . \ldots . \ldots . \ldots 93$

5.4 Axial symmetry breaking . . . . . . . . . . . . . 105

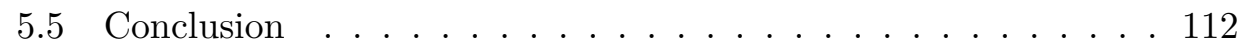

\begin{tabular}{lll}
\hline II Colloidal ouzo drops & 115
\end{tabular}

$\begin{array}{lll}6 & \text { Colloidal ouzo drops } & 117\end{array}$

6.1 Introduction . . . . . . . . . . . . . . . . . . . . . 118

6.2 Methods and results . . . . . . . . . . . . . . . . . . . . 119

6.3 Conclusion and outlook . . . . . . . . . . . . . 126

6.4 Method details and supplementary information . . . . . . . 127

\begin{tabular}{lll}
\hline III & Surface nanodroplets & 129
\end{tabular}

$\begin{array}{lll}7 & \text { Surface nanodroplets formation } & 131\end{array}$

7.1 Introduction . . . . . . . . . . . . . . . . . . . . . 131

7.2 Results and discussion . . . . . . . . . . . . . . . . . . 134

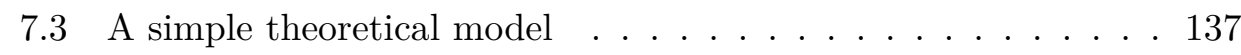

7.4 Buoyancy driven convection effects . . . . . . . . . . . . . . 139

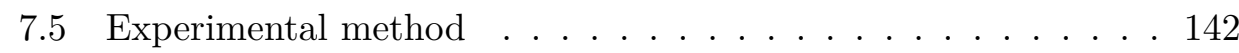

7.6 Conclusions . . . . . . . . . . . . . . . . . . . . . . 144

8 3D fitting procedure for nanodroplets $\quad 145$

8.1 Introduction . . . . . . . . . . . . . . . . . . 146

8.2 Detailed 3D fitting procedure . . . . . . . . . . . . . . 148

8.3 Evaluation of the effects from cut-off threshold . . . . . . . . . 150

8.4 3D-SCFP versus 2D fitting . . . . . . . . . . . . . 151

8.5 Conclusion $\ldots \ldots \ldots \ldots \ldots \ldots \ldots \ldots$

8.6 Appendixes . . . . . . . . . . . . . . . . 156

9 Conclusions and outlook $\quad 159$

$\begin{array}{ll}\text { References } & 165\end{array}$ 
Summary $\quad 185$

$\begin{array}{ll}\text { Samenvatting } & 187\end{array}$

$\begin{array}{ll}\text { Scientific output } & 189\end{array}$

\begin{tabular}{ll}
\hline Acknowledgements & 193
\end{tabular} 



\section{Introduction}

\subsection{Motivations}

We live in a multi-scale world. Dynamics of fluids on large scales affect our planet, while on small scales they are significantly related to our life as well. Droplets are one of the most common forms of fluids in small size. They have beautiful spherical profiles, which is so attractive that, in our daily life, there are innumerable beautiful pictures of them created by photographers. Droplets are essential in scientific research as well, as a significant amount of advanced technologies in our modern life relate to these small droplets (Fig.1.1): Inkjet technology, which helps us print newspapers, books, and posters we read every day and even the complicated electronics of the cell phones in our hands, is about creating small aqueous droplets at super high speeds and drying them into controlled patterns [1-4]. In cosmetic, food, and medicines industries, emulsion droplets are primary ingredients of the products. It is critically important to know how to create droplets on sub-micron or nano scales, how to make them stable, and how to have different materials capsuled inside [5, 6]. Also, by using droplets, scientists or engineers can achieve DNA mapping in biology and chemical extraction in chemistry [7 9]. Nevertheless, droplets can be dangerous as well, although they are small. When aircraft fly through clouds, the water droplets will impact the aircraft wings. If the droplets did not shed and stayed there, they would freeze with tragic consequences.

In the past decades, numerous efforts have been made by many scientists from different fields including physics, chemistry, biology, environmental sci- 


\subsection{MOTIVATIONS}

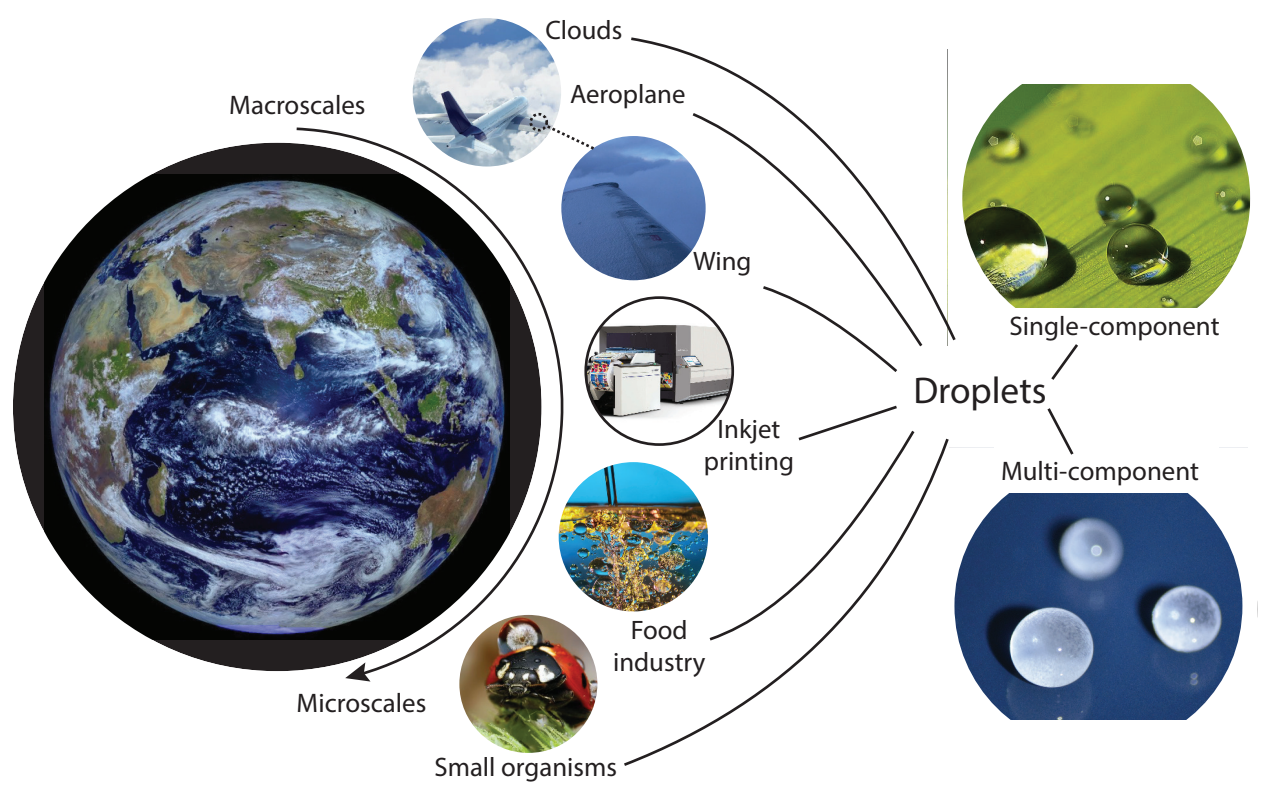

Figure 1.1: The ubiquity of small-scale droplets: For example, the formation of clouds, freezing issue of airplane wings, inkjet technology, food industry, the life of small organisms all relate to droplets, either single component droplets or multi-component droplets. (source for images: earth (NASA website); aeroplane (Shutterstock website); inkjet printer (Océ website); multi-component droplets image (took in lab); other images(Pinterest website)).

ence, material science, and engineerings, to understand droplets behaviours in different conditions for different purposes [9 13]. Up to now, many studies have been done on the physical behaviors of droplets of pure liquids, liquids with dispersed particles, or even binary liquid mixture, i.e. still in single liquid systems or binary liquid systems. The dynamical behaviors of evaporating and dissolving droplets in multicomponent (more than two components) systems, however, have much less been studied, although they are more frequently encountered in practice. We know that the fluid composition determines a number of its physical properties including surface tension, solubility, nonequilibrium and so on, and the flow of the fluid can vary its composition locally. In the evaporating or dissolving droplets, how the diffusion-deduced property variations interact with the hydrodynamics, how this two-way interactions affect the dynamical behaviors of the droplets, how the interactions are affected by the ambient conditions, and what the unexpected phenomena would appear, are still unknown. As the development of modern science and technology, to thoroughly understand these concepts in multicomponent droplets is becoming more and more critical, in particular to advanced manufacturing. Here, we will give our contributions to this issue by studying the evaporation and dissolution of droplets in ternary systems. 


\subsection{Small-scale world of droplets}

Droplets exist in a small-scale world. In this small-scale world, the small dimensions suppress some physical phenomena prevailing on large scales, while some other physical phenomena overlooked on large scales become pronounced [14. The competition relationships of small scale forces determine the features of small-scale droplets.

For instance, on small scales, pure droplets always have perfectly rounded profiles statically, because the excess pressure by surface tension (Laplace pressure) can dominate the reduced hydrostatic pressure on small scales. When the droplets are smaller than a critical length scale, the so-called capillary length $\ell_{c}=\sqrt{\gamma /(\rho g)}$ (surface tension $\gamma$, liquid density $\rho$, and earth's gravity $g$ ), the cohesive force of the surface tension pulls the droplets into spherical shapes. Whereas concerning the hydrodynamics inside the droplets, the inertial nonlinearity, which plays a critical role in enriching large-scale systems, is suppressed by the droplets dimensions. Usually, the typical Reynolds number $R e=U L / \nu$ for the evaporating or dissolving droplets is less than 10 , since the droplets have a characteristic length scale $L$ in the order of a millimeter or less, and a characteristic velocity scale $U$ inside in the order of up to $1 \mathrm{~mm} \mathrm{~s}^{-1}$.

The flow within the droplets can display quite rich behavior, because other physical phenomena, which can be ignored and less familiar on the macroscale, acquire prominence here. The dominance of these "unfamiliar" physical phenomena brings its distinguishing features on small scales. Here, we will give a brief discussion on those of them that are essential to the topic of this thesis.

\subsubsection{Marangoni flows}

In a variety of small-scale processes, surface tension dominates gravity. It can be explained by Bond number $B o$, which prescribes the relative importance of forces induced by gravity and surface tension $\gamma$, and has an expression as

$$
B o=\frac{\rho g L^{2}}{\gamma} .
$$

The above formula indicates that the surface tension becomes more dominant as the decrease in system size. Thus, the flows induced by (interfacial) surface tension gradients, i.e., Marangoni flows, become ubiquitous and vital on small scales.

Surface tension (also called interfacial tension) results from an imbalance of attractive intermolecular forces at the fluid-gas interface (or immiscible liquidliquid interface). So either locally changing temperature or fluid composition can lead to the change of surface tension. Once there is a temperature gradient along the surface, the resulting surface tension gradient would cause a 
(a) Thermal Marangoni flow
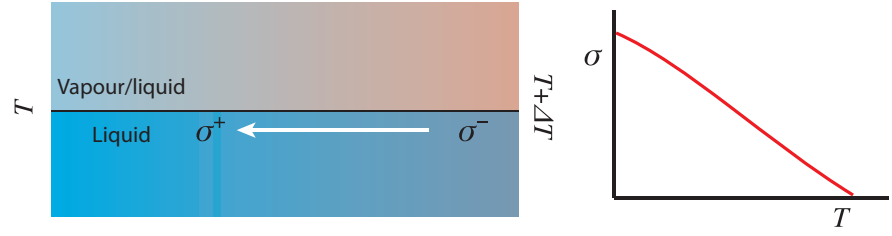

(b) Solutal Marangoni flow
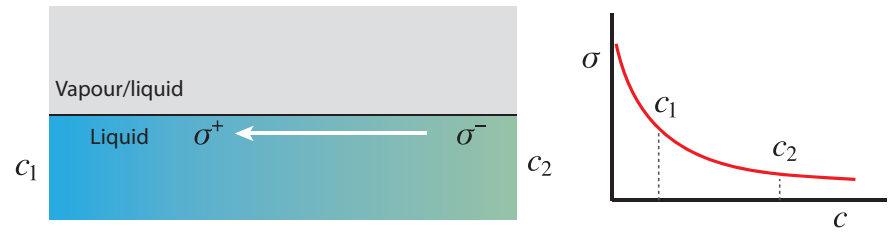

(c) Surfactant-induced Marangoni flow
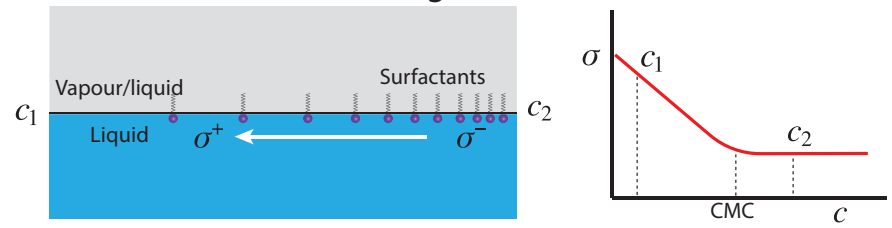

Figure 1.2: Illustrations of Marangoni flows driven by (interficial) surface tension difference: (a) Thermal Marangoni flow; (b) Solutal Marangoni flow; (c) Surfactant-induced Marangoni flow.

tangential flow along the surface, named thermal Marangoni flows (Fig. 1.2 a). In general, thermal Marangoni flows toward the low-temperature side, as a warmer gas phase with more liquid molecules reduces surface tension; different liquids have different surface tensions. So fluids with concentration gradients along the surface can generate surface tension gradients as well, and the induced flows are called solutal Marangoni flows (Fig.1.2b). Another type of Marangoni flow is surfactant-induced Marangoni flow. Surfactants are compounds with an unusual structure (often with a hydrophilic head/ and hydrophobic tail) that make them reside at the free surface or interface in an energetically favorable state. Thus, surfactants can lower the (interfacial) surface tension as well, leading to Marangoni flows (Fig.1.2k).

\subsubsection{Pronounced diffusion processes}

Another phenomenon which comes to prominence on small scales is the diffusion process. The dimensionless number Péclet number Pe expresses the relative importance of convection to diffusion and is governed by the Péclet

$$
P e=\frac{L U}{D},
$$


where $D$ is mass diffusion coefficient. This number expresses that the diffusion transport competes with or advective transport. On the macroscale the diffusive transport rate is minimal, implying $R e \gg 1$.

The relevance of diffusion implies the variation of concentration field. Once there is a concentration difference along the droplet surface or interface, solutal Marangoni flows will appear and therefore affect the diffusion process in turn. It implies that diffusion can induce complicated dynamics on small scales. A small droplet, for example, can be self-propelled by this Marangoni stress when the viscosity ratio of the droplet liquid to the surrounding fluid is lower than the length scale ratio of the droplet size to the solutal interactive length scale [15].

Another interesting phenomenon induced by diffusion is spontaneous emulsification. The diffusion causes local supersaturation of one species in a mixture, giving rise to nucleation and growth of microdroplets of this species (see sec.1.4). This mechanism is called "diffusion and stranding," which was discussed by Davies and Rideal 60 years ago [16]. These nucleated microdroplets can enrich the flow as well, causing some interesting and complicated dynamical behaviors. In the Part $[I$ of this thesis, we will explore how the diffusion processes, i.e., evaporation or dissolution, affect the hydrodynamics in the multicomponent droplets based on this "diffusion and stranding" mechanism.

For an immersed multicomponent droplet in a liquid, if all the components in the droplet are sparingly soluble in the surrounding fluid, then the diffusion process can be regarded as a one direction process, i.e., from the droplet inside towards the outside [17]. If one of the droplet components is miscible with the surrounding liquid, then this assumption is not applicable anymore and we have to consider it as a multi-diffusion process. Across the droplet interface, the miscible droplet component diffuses out, and simultaneously, the surrounding fluid diffuses into the droplet. For the diffusion processes at both sides of the interface, we can apply Fick's diffusion law, i.e.,

$$
\begin{aligned}
\frac{\partial m_{\alpha}^{\mathrm{d}}}{\partial t} & =D_{\alpha}^{\mathrm{d}} \frac{\partial^{2} m_{\alpha}^{\mathrm{d}}}{\partial x^{2}}, \\
\frac{\partial m_{\alpha}^{\mathrm{h}}}{\partial t} & =D_{\alpha}^{\mathrm{h}} \frac{\partial^{2} m_{\alpha}^{\mathrm{h}}}{\partial x^{2}},
\end{aligned}
$$

where the superscripts indicate the side, i.e., either drop side (d) or host liquid side (h), the subscript the component $\alpha, x$ position, $t$ time, $m_{\alpha}$ the mass fraction, and $D_{\alpha}$ diffusivity. To balance the mass transport at the moving interface $s(t)$, we have,

$$
\left(m_{\alpha}^{\mathrm{h}}-m_{\alpha}^{\mathrm{d}}\right) \frac{\mathrm{d} s}{\mathrm{~d} t}=D_{\alpha}^{\mathrm{d}} \frac{\partial m_{\alpha}^{\mathrm{d}}}{\partial x}-D_{\alpha}^{\mathrm{h}} \frac{\partial m_{\alpha}^{\mathrm{h}}}{\partial x}, \quad \text { when } x=s(t) .
$$


Therefore, the problem becomes a so-called Stefan problem, following the mathematical terminology of boundary value problems for a partial differential equation [18]. However, phase separation is still out of consideration in above equations. It is necessary to integrate the liquid-liquid equilibrium process at the interface into the equations. We will explore this issue in chapter 4

\subsubsection{Surface/interface-related phenomena}

Since droplets have rounded profiles and small volume sizes, their surface-tovolume ratios are relatively large. As a consequence, the surface/interface effects have great impact on the statics and the dynamics of the droplets. The essentials of most dynamical behaviors of droplets are related to the surfaces, such as wetting [19, 20], drop impact [21, 22], condensation [23, levitating droplets [24], droplet formation in a microchannels [25, 26], the Leidenfrost effect [27], the Marangoni effect (see sec.3.5.2), diffusiophoresis [28, 29], electrophoresis [30, 31], and so on. Here, we will focus on the primary surface effects that have influences on the evaporation and dissolution of droplets.

(a)

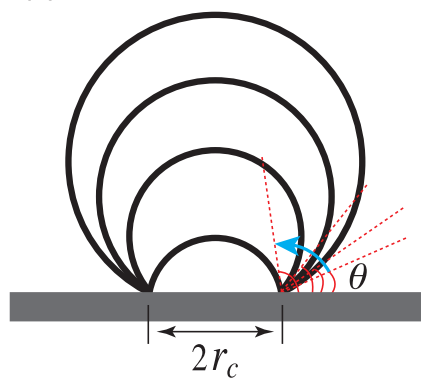

(b)

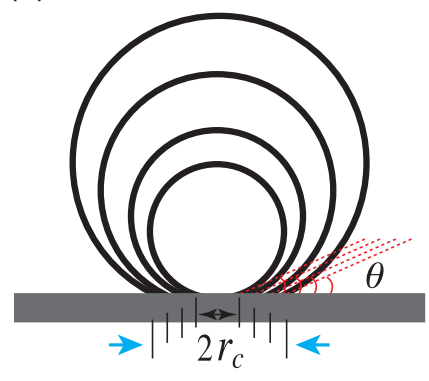

Figure 1.3: Scheme of a shrinking droplet in (a) constant contact area mode or (b) constant contact angle mode.

The surfaces/interfaces of the droplet system could be liquid-solid surface, liquid-vapor interface, or liquid-liquid interface. We first talk about the effects caused by the liquid-solid surface. When a droplet sits on solid surfaces, it can have different Young's contact angles for the different surface materials. For different contact angle values, the liquid-vapor surfaces/liquid-liquid interfaces have different geometries. The diffusion process across these surfaces/interfaces is confined by different boundary conditions, resulting in different evaporating/dissolving flux distributions along the surfaces/interfaces (see sec.1.3). Consequently, the droplet can have different mass loss rates and different inside flow pattern on different surfaces [32-35]. Beside, the defects (pinning points) on the solid surface can cause a sticky contact line of the 
droplet. Then the droplet evaporates or dissolves in a constant-contact-area mode (Fig. 1.3a) instead of constant-contact-angle mode (Fig. 1.3b) [36, 37]. The pinned contact line can further cause capillary flows inside the droplet, which play an essential role in the famous "coffee stain effect" [38, 39].

The liquid-vapor equilibria at the liquid-vapor surface and the liquid-liquid equilibria at the liquid-liquid interface govern the evaporation and dissolution processes. For multicomponent droplets, it is inevitable to consider the interactions between different species in the equilibria process. In liquid-vapor equilibrium, these interactions can be expressed by Raoult's law (see sec.1.3.2. Also, the condensation of the vapor at the surface can be neglected compared to the evaporation of the liquid. However, in the liquid-liquid equilibrium, diffusion processes towards both directions, i.e., multi-diffusion process, must be considered. The phase equilibria are achieved by applying the condition of equality of the chemical potentials with the adoption of some activity coefficient model (UNIQUAC, UNIFAC, et al.). The detailed discussions are given in chapter 4

\subsection{Evaporation dynamics of droplets}

\subsubsection{Single-component droplets}

(a)

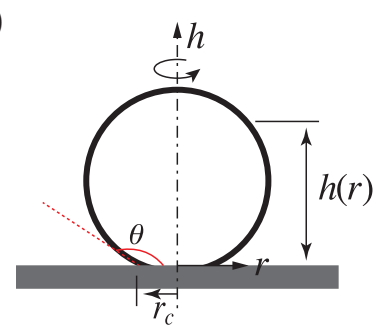

(b)

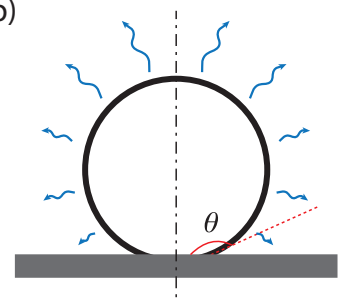

(c)

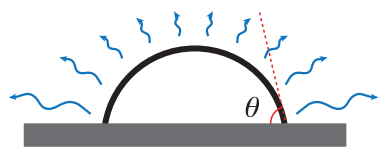

Figure 1.4: (a) Schematic illustration of the evaporation model. (b) respective (c) illustrate the distributions of evaporation flux along droplet surface for low wettability $\left(\theta>90^{\circ}\right)$ respective high wettability $\left(\theta<90^{\circ}\right)$.

There are several models proposed to describe the evaporation process of droplets [35, 40]. The classical theory for quasi-steady natural evaporation of single component droplets was proposed by Popov [32]. The model predicts the rate of mass loss in terms of the contact angle by solving the diffusion process in a nonuniform vapor concentration field around the droplet without consideration of thermal and free-convection effects. The evaporation flux $J(r)$ 
on the surface is given by 32 ]

$$
\begin{aligned}
& J(r)=\frac{D_{\mathrm{air}}^{\mathrm{vap}}\left(c_{\mathrm{sat}}-c_{\infty}\right)}{r_{c}}\left[\frac{1}{2} \sin \theta+\sqrt{2}(\cosh \alpha+\cos \theta)^{3 / 2}\right. \\
& \left.\times \int_{0}^{\infty} \frac{\cosh \theta \tau}{\cosh \pi \tau} \tanh [(\pi-\theta) \tau] P_{-1 / 2+i \tau}(\cosh \alpha) \tau \mathrm{d} \tau\right],
\end{aligned}
$$

where

$$
r=\frac{r_{c} \sinh \alpha}{\cosh \alpha+\cos \theta}
$$

is the radial coordinate at the surface of the droplet, $\alpha$ and $\beta$ are toroidal coordinates, $r_{c}$ is the contact radius of the droplet, $D_{\text {air }}^{\mathrm{vap}}$ is the coefficient of vapor diffusion, $c_{\mathrm{sat}}$ is the saturated vapor concentration on the droplet surface, $c_{\infty}$ is the concentration of vapor at infinity, and $\theta$ is the contact angle (Fig.1.4a). As a result of equation (1.6), there is a divergence of the local evaporation flux at the contact line when $\theta<90^{\circ}$, while the divergence is absent when $\theta>90^{\circ}$ (Fig. $1.4 \mathrm{p}$ respective c).

The evaporation rate $\dot{m}$ of the mass can be expressed as an integral of the evaporation flux over the droplet surface $-\int_{0}^{r_{c}} J(r) \sqrt{1+\left(\partial_{r} h(r)\right)^{2}} 2 \pi r \mathrm{~d} r$, i.e.,

$$
\dot{m}=-\pi r_{c} D_{\text {air }}^{\mathrm{vap}}\left(c_{\mathrm{sat}}-c_{\infty}\right) f(\theta),
$$

where

$$
f(\theta)=\frac{\sin \theta}{1+\cos \theta}+4 \int_{0}^{\infty} \frac{1+\cosh 2 \theta \tau}{\sinh 2 \pi \tau} \tanh [(\pi-\theta) \tau] \mathrm{d} \tau .
$$

\subsubsection{Multi-component droplets}

The fundamental difference between a pure liquid and a mixture is the vaporliquid equilibrium. Whereas in the case of a pure fluid $i$ the vapor concentration $c_{i}$ directly above the liquid-air interface is saturated (i.e., $c_{i}=c_{\text {sat }}$ ), it is lower for the case of mixtures. At the liquid-gas interface, the vapor-liquid equilibrium according to Raoult's law obeys

$$
c_{i, \mathrm{VLE}}=\Gamma_{i} x_{i} c_{i, \mathrm{sat}} .
$$

By virtue of the above equation, the evaporation rates are coupled with the local droplet composition at the interface via the liquid mole fraction $x_{i}$ and the activity coefficient $\Gamma_{i}$. Therefore, the mass loss rate of each component $i$ in an multicomponent droplet (well mixed) can be expressed as

$$
\dot{m}_{i}=-\pi r_{c} D_{i, \text { air }}^{\text {vap }}\left(c_{i, \mathrm{VLE}}-c_{i, \infty}\right) f(\theta),
$$


and the evaporation rate of the droplet mass $\dot{m}$ is given by

$$
\dot{m}=\sum \dot{m}_{i},
$$

where the subindex $i$ stands for the $i$ component.

\subsection{The "ouzo effect" and ternary phase diagram}

Liquid-liquid phase equilibria are essential dynamical behaviors existing in the multicomponent fluids system. For instance, the "ouzo effect", a well-known daily life phenomenon, enables spontaneous emulsification without the use of surfactants or the need for external energy input. In this thesis, by studying the ouzo droplets, we will demonstrate that liquid-liquid phase equilibria are worthy of attention in the small droplet system. Here, we will have a brief discussion on the "ouzo effect."

\subsubsection{The "ouzo effect"}

Ouzo is an anise-flavored aperitif, primarily consisting of water, ethanol and a small amount of anise oil. When water is added to ouzo, the dissolved anise oil spontaneously nucleates into many microdroplets. The solution instantaneously appears milkily white since the nucleated microdroplets scatter light. Vitale and Katz [41, 42] termed this physical phenomenon the "ouzo effect." This phenomenon has triggered much interest in scientific \& engineering community due to its potential applications in food industry, cosmetics, and chemical industry [43,46].

Ethanol, which is not only soluble in water but also in oil, plays a role as co-solvent in the ouzo solution. Ethanol molecules tend to surround the water-insoluble oil, and ethanol molecules themselves are miscible with water. The ouzo solution (water, ethanol, and oil) as a whole can be miscible, thus appearing transparent. However, once the co-solvent fraction in the solution is decreased, for example, by adding water, there will not be enough excess ethanol molecules surrounding oil, leading to the separating-out of oil. Under some conditions (see below), the oil separates out into many emulsion droplets, i.e., the so-called the "ouzo effect." However, up to now, there is still not a complete understanding on the detailed mechanisms and dynamics of this process, although there are some proposed mechanisms responsible for the spontaneous emulsification, like interfacial turbulence or "diffusion and stranding" [47, 48].

We can obtain insight into this spontaneous emulsification in the perspective of thermodynamic equilibrium by studying the process when two immiscible liquids contact to have an emulsion state. There is no external energy 
input during the process. According to the first law of thermodynamics, the infinitesimal change in the Gibbs energy $(G=U+P V-T S)$ of the system $d G$ has an expression as

$$
d G=\gamma d A-T d S .
$$

The first term in the right-hand $\gamma d A$ is the energy change due to interface expansion ( $\gamma$ interfacial tension, $d A$ interface variation), while the second term $T d S$ comes from the increment of entropy $S$ at constant temperature and pressure. The second law of thermodynamics states that: $d G>0$ means the system is in equilibrium, whereas $d G \leq 0$ indicates that the system is not in equilibrium and spontaneous emulsification is possible. Whether the system is in equilibrium or not depends on how the composition of the solution is varied. People usually use a ternary phase diagram to depict the composition variation of the ternary system.

\subsubsection{Ternary phase diagram}

In this section, we will give a brief introduction to the ternary phase diagram. The ternary phase diagram is a convenient tool to depict the composition of mixtures containing three different liquids in a equilateral triangular diagram.
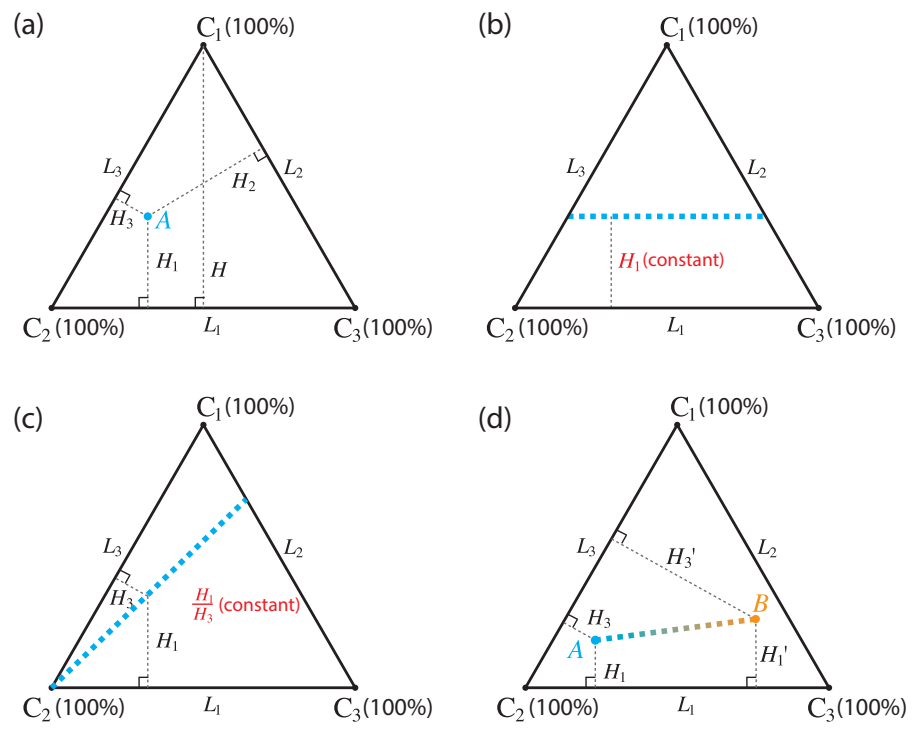

Figure 1.5: Properties of equilateral triangular diagram.

In the equilateral triangular diagram (Figure 1.5), the three corners $C_{1}$, $C_{2}$ and $C_{3}$ stand for three different species $i(i=1,2,3)$. The ratios of three species in the mixture are mapped to different positions on this tri- 
angle (Fig.1.5 ). For each species $i$, its mass (or volume, or mole) fraction $x_{i}$ is determined by the ratio of the perpendicular distances from the opposite line $H_{i}$ to the length of the triangle altitude $H$, i.e., $x_{i}=\frac{H_{i}}{H}$. Thus, its fraction is $100 \%$ at a corner $C_{i}$ and $0 \%$ on the opposite line $L_{i}$. According to Viviani's theorem, i.e., $H=H_{1}+H_{2}+H_{3}$, for each position $A$ (Fig.1.5a) on the ternary phase diagram, the proportions of the three species sum to $100 \%$. Besides, several other useful properties of the equilateral triangle diagrams can be deduced and are given below [49],

(i) On any line (blue dashed line in Fig. $1.5 \mathrm{~b}$ ) parallel to line $L_{i}$, the concentration of the species of the opposite corner $C_{i}$ is constant;

(ii) On any line (blue dashed line in Fig.1.5k) connecting a corner $C_{i}$ of the species $i$ with a point $P_{i}$ on the opposite line $L_{i}$, the ratio of the other two components (not $i$ ) is constant;

(iii) When two solutions with compositions $\mathrm{A}$ and $\mathrm{B}$ are mixed with any ratios, the compositions of the mixtures lie on the straight line $\mathrm{AB}$ in the equilateral triangle diagram (Fig. $1.5 \mathrm{~d}$ ).

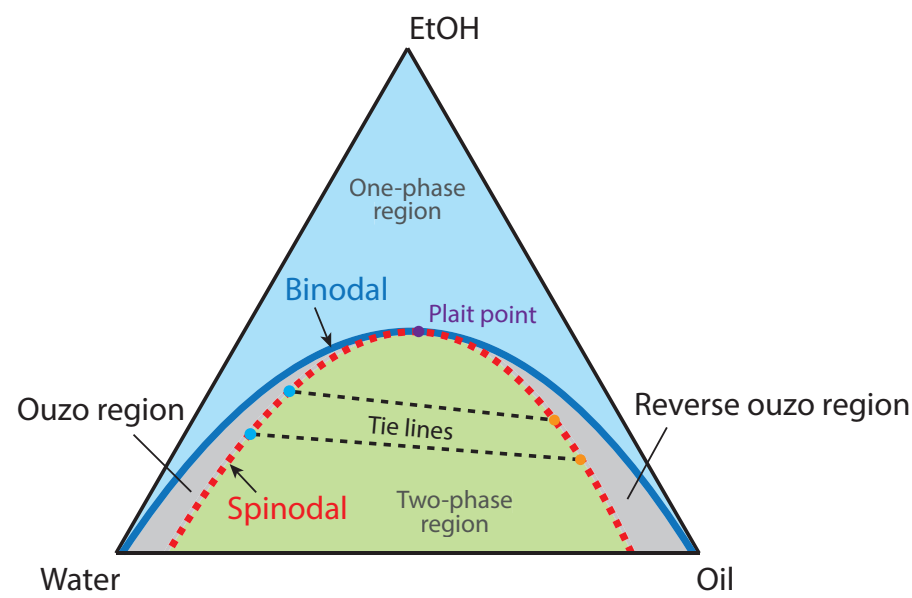

Figure 1.6: The typical ternary phase diagram of water-ethanol-oil system.

Above the equilateral triangle, phase curves (binodal curve and spinodal curve) determine the ternary phase diagram for a certain ternary system (Figure 1.6). The curves have a dependence on temperature and pressure. Above the binodal curve, it is an one-phase region (a stable region). Once mixtures have overall compositions enter inside the binodal curve, they will split into oil-riched phase and water-riched phase. Tie lines connect composition positions of the oil-riched phase and water-riched phase in the triangle. Any 
mixture with an overall composition along a tie line gives the same oil-riched phase and water-riched phase compositions. The multiphase region consists of a metastable region and an unstable region (two-phase region), which are separated out by the spinodal curve. The "ouzo effect" takes place in the metastable region, which includes the standard ouzo region in water-rich side, generating oil emulsions, and the reverse ouzo region in oil-rich side, leading to water emulsification. The binodal curve and the spinodal curve intersect at a plait point.

\subsection{A guide through this thesis}

\subsubsection{Introducing the "ouzo effect" into droplets}

The "ouzo effect" has been brought into small-scale devices in the solvent exchange process, through which we can generate a large number of nanodroplets on a surface. In this process, the mixture of oil and oil-soluble solvent first covers a hydrophobic substrate in a narrow channel, and then the mixture is replaced by an oil-insoluble solution. When these two solutions meet, the oil solubility reduction leads to the nucleation of nanodroplets not only in the bulk liquid (the standard "ouzo effect") but also on the substrate. The flow conditions during the solvent exchange affect the formation of surface nanodroplets (part III).

The evaporating/dissolving droplets are very suitable systems to exploit the consequent physical phenomena induced by the "ouzo effect". In these systems, diffusion process can act as a trigger of the"ouzo effect." The absence of the inertial nonlinearity reduces the complexity of the flow structures, providing an opportunity to study the hydrodynamics caused by the "ouzo effect." The competition between small-scale phenomena keeps the richness in the system. The influence of the surface effects on the droplet evaporation and dissolution provides potentials to manipulate the dynamics in the system, such as to vary the preferred location of the "ouzo effect," to alter the flow patterns, or to have

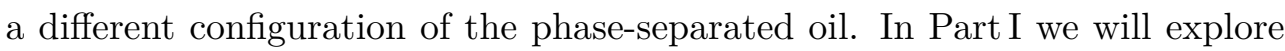
this subject. 


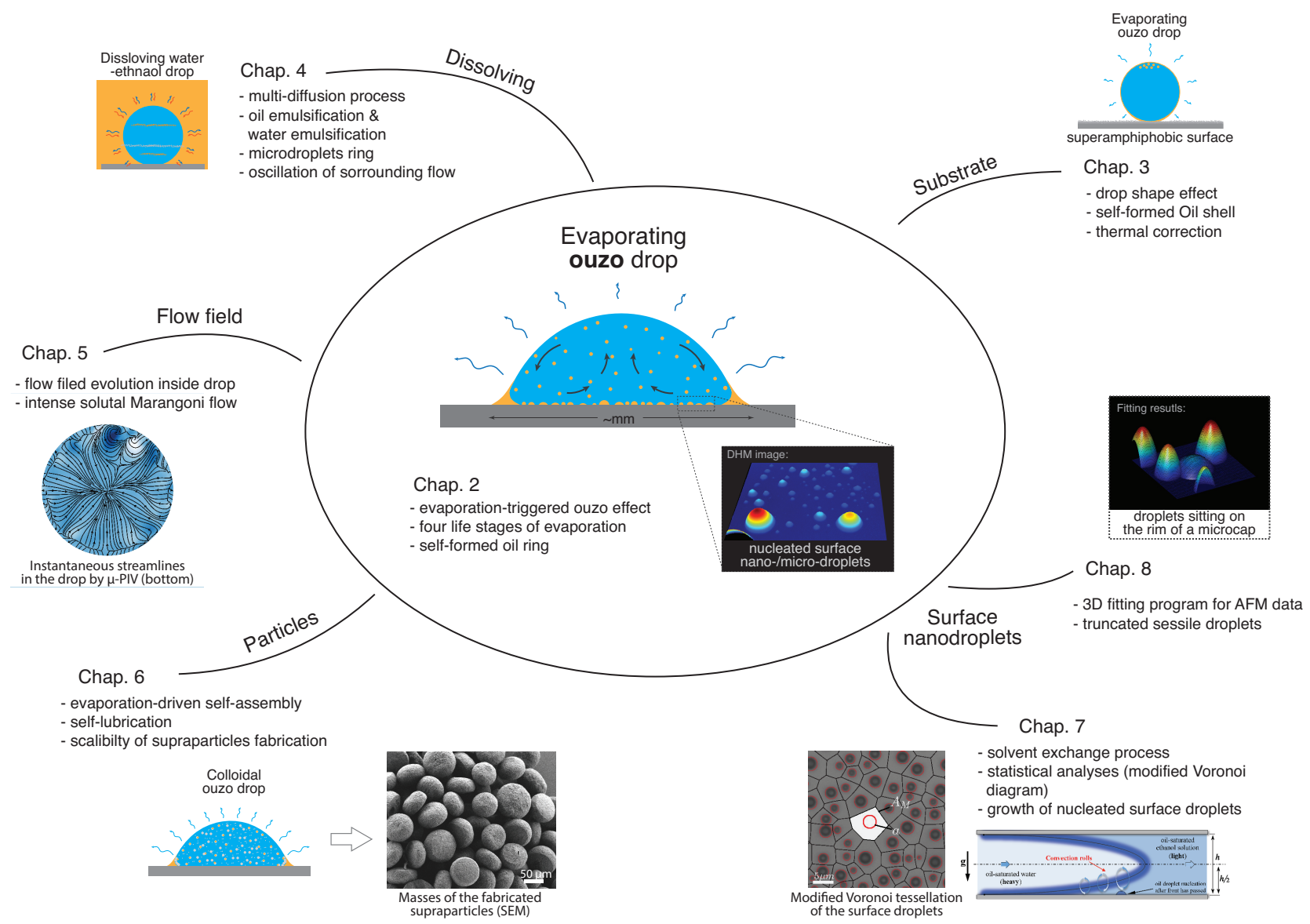

Figure 1.7: The diagram illustrating the relationship among different chapters, together with the listed highlights of each chapter for this 


\subsubsection{Outline of the thesis}

This thesis is organized as follows. We first open our story in Part] by answering a simple and interesting question: What happens when an ouzo droplet is evaporating? We will study the evaporating sessile ouzo droplets on a hydrophobic surface in $\S 2$ and a spherical ouzo drop on a superamphiphobic surface in $\S 3$. In these two chapters, we will demonstrate how the "ouzo effect" is triggered by the evaporation process on the surface of droplets. Through the combination of experimental and numerical methods, we will gain more quantitative insight into the whole evaporating process. Based on our understanding acquired from the experiments and simulations, we will further propose a generalized diffusion model to predict the evaporative mass loss rate of the evaporating multicomponent droplets. The comparison of the results in these two chapters will reveal that changing the substrate can cause different phenomena in the evaporating ouzo drops. In $\S 4$, we will investigate the dissolution of an immersed ouzo drop and highlight the importance of multi-diffusion processes. By integrating multi-diffusion process theory and liquid-liquid equilibrium theory, we will propose a one-dimensional model to explain the observed experimental phenomena. In the last chapter of this part (§5), we will have a systematic investigation on the evaporating processes of pure, binary, and ternary sessile droplets, as well as the complicated flow fields in the evaporating multicomponent droplet, numerically and experimentally. Besides, there will be many further interesting phenomena presented in this part.

With the deep understanding of the physical phenomena from Part] in Part III we will give a practical application of evaporating ouzo droplets. By adding nanoparticles into the ouzo solutions, we will create colloidal ouzo droplets for supraparticle synthesis. In $\S 6$, we will propose a facile and robust approach, through which the commonly-used hydrophobic surfaces are applicable for evaporation-driven particles assembly. Moreover, the fabrication of high-porosity supraparticles with controllable shapes is feasible by changing the initial ratio of oil to nanoparticles in the colloidal drops. We expect this application will give a demonstration for the application of the ouzo droplets studies in Part!

In Part III, we will focus on the nucleated surface nano-/micro-droplets by the solvent exchange method. Here, the "ouzo effect" is induced in a narrow channel under well-controlled flow conditions. Thereafter, the droplet nuclei on the surface are exposed to an oil oversaturated environment, which drives the formation of surface nanodroplets, i.e., by a non-standard "ouzo effect." In $\S 7$, we will statistically analyze the volume of the nucleated nanodroplets, and propose a theoretical model to explain the statistical relationship between the 
volume of the nucleated nanodroplets and the Péclet number of the flow. In $\S 8$, we will develop a comprehensive $3 \mathrm{D}$ spherical cap fitting procedure for the accurate extraction of the morphologic characteristics of nanodroplets from atomic force microscopy (AFM) topographic images, which makes it possible to perform a statistical analysis of the mass distribution of nanodroplets. The thesis is concluded in $\S 9$. 



\section{Part I}

\section{Ouzo drops}





\section{2}

\section{Evaporating flat ouzo drops*}

The evaporation of an Ouzo droplet is a daily-life phenomenon, but the outcome is amazingly rich and unexpected: Here we reveal the four different phases of its life with phase transitions in between, and the physics which governs this phenomenon. The Ouzo droplet may be seen as model system for any ternary mixture of liquids with different volatilities and mutual solubilities. Our work may open up numerous applications in (medical) diagnostics and in technology, such as coating or for the controlled deposition of tiny amounts of liquids, printing of LED or OLED devices, or phase separation on a sub-micron scale.

\subsection{Introduction}

Evaporating liquid droplets are omnipresent in nature and technology, such as in inkjet printing, coating, deposition of materials, medical diagnostics, agriculture, food industry, cosmetics, or spills of liquids. While the evaporation of pure liquids, liquids with dispersed particles, or even liquid mixtures has intensively been studied over the last two decades, the evaporation of ternary mixtures of liquids with different volatilities and mutual solubilities has not yet been explored. Here we show that the evaporation of such ternary mixtures can trigger a phase transition and the nucleation of microdroplets of one of

${ }^{*}$ Based on: H. Tan, C. Diddens, P. Lv, J.G.M. Kuerten, X. Zhang, and D. Lohse, Evaporation-triggered microdroplet nucleation and the four life phases of an evaporating Ouzo drop, Proceedings of the National Academy of Sciences 113, 8642-8647 (2016).

${ }^{\dagger}$ Numerical work performed by C. Diddens; Confocal images took by P. Lv \& H. Tan. 
the components of the mixture. As model system we pick a sessile Ouzo droplet (as known from daily life - a transparent mixture of water, ethanol, and anise oil) and reveal and theoretically explain its four life phases: In phase I, the spherical cap-shaped droplet remains transparent, while the more volatile ethanol is evaporating, preferentially at the rim of the drop due to the singularity there. This leads to a local ethanol concentration reduction and correspondingly to oil droplet nucleation there. This is the beginning of phase II, in which oil microdroplets quickly nucleate in the whole drop, leading to its milky color which typifies the so-called 'Ouzo-effect'. Once all ethanol has evaporated, the drop, which now has a characteristic non-spherical-cap shape, has become clear again, with a water drop sitting on an oil-ring (phase III), finalizing the phase inversion. Finally, in phase IV, also all water has evaporated, leaving behind a tiny spherical cap-shaped oil drop.

A coffee drop evaporating on a surface leaves behind a roundish stain [38]. The reason lies in the pinning of the drop on the surface, together with the singularity of the evaporation rate at the edge of the drop, towards where the colloidal particles of the drop are thus transported. This so-called 'coffeestain-effect' has become paradigmatic for a whole class of problems, and nearly 20 years after Deegan et al. [38 presented it to the scientific community, still various questions are open and the problem and its variations keep inspiring the community [7, 32, 33, 35, 38, 50,62],

What happens when an Ouzo drop is evaporating? The Greek drink Ouzo (or the French Pastis or the Turkish Raki) consists of an optically transparent ternary mixture of water, ethanol, and anise oil. When served, water is often added, leading to the nucleation of many tiny oil droplets, which give the drink its milky appearance. This is the so-called Ouzo-effect [41]. As we will see in this paper, also this problem can become paradigmatic, due to its extremely rich behavior, now for the evaporation-triggered phase separation of ternary liquids and droplet nucleation therein.

The reason for the Ouzo effect lies in the varying solubility of oil in ethanolwater mixtures: With increasing water concentration during the solvent exchange (i.e., water being added), the oil solubility decreases, leading to droplet nucleation in the bulk and - if present - also on hydrophobic surfaces (so-called surface nanodroplets) [37, 63. 

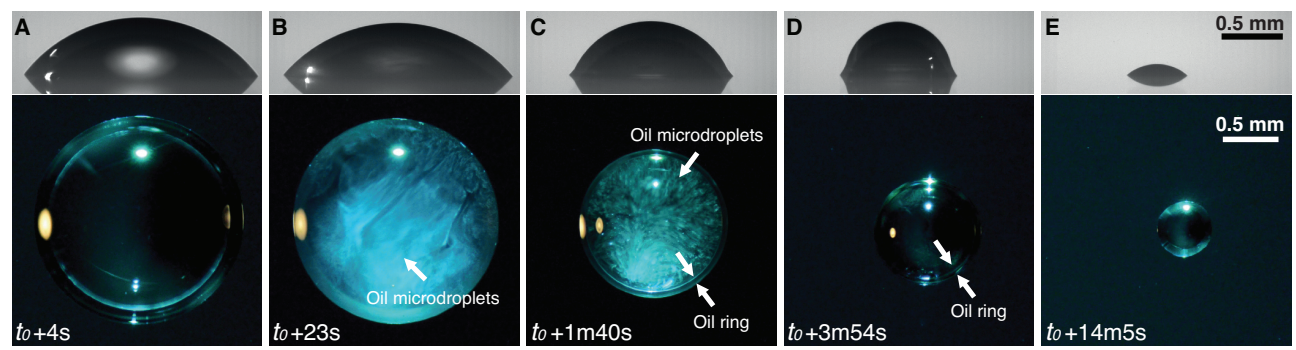

Figure 2.1: Experimental snapshots during the evaporation of an 'Ouzo' drop on a flat surface. The initial volume of the drop is $0.7 \mu \mathrm{L}$ with an initial composition of $37.24 \%$ water, $61.06 \%$ ethanol and $1.70 \%$ anise oil (a mixture we refer to as 'Ouzo') in terms of weight fractions. The time $t_{0}$ is defined as the moment the needle was pulled out of the drop. A time series of the evaporation process can be seen in Videos S1 and S2. (A) At early times, the Ouzo drop is transparent and has a spherical-cap shape. The light ring and spots in the top view image are caused by reflection and refraction of the light source. (B) A color transition arises as a result of the Ouzo effect, i.e. the nucleation of nano- to micro-sized oil droplets, which are convected by the flow inside the Ouzo drop. The scattering of light at the nucleated microdroplets leads to the milky coloring of the drop. (C) The Ouzo drop loses its spherical cap shape due to the appearance of an oil ring. The complex transitions from (A) to (C) happen within two and a half minutes, a short time compared to the whole process. (D) The Ouzo drop is transparent again. Oil microdroplets in the bulk grow big enough to sit on the surface or directly merge with the oil ring by convection. (E) After around 14 minutes of evaporation, only anise oil is left, now in a spherical cap shape again.

\subsection{Results and discussion}

\subsubsection{Series of events during evaporation of a sessile Ouzo drop and their interpretation}

When an Ouzo drop is evaporating, this Ouzo effect is locally triggered by the preferred evaporation of the more volatile ethanol as compared to the less volatile water and the even less volatile oil. As the evaporation rate is highest at the rim of the drop [35, we expect the oil microdroplets to nucleate there first. Indeed, this is what we see in our experiments, in which we have deposited a $\mu L$ Ouzo drop on a transparent hydrophobic octadecyltrichlorosilane (OTS)-glass surface, monitoring its evaporation under ambient conditions with optical imaging synchronized from the top and side (Fig. 2.1 experimental setup sketch see Fig. 2.8 ), from the bottom (Fig. 2.2 ) and confocally (Fig. 2.3). For an illustration of the evaporation process see Figure 2.4. At early times, the Ouzo drop is transparent and has a spherical cap shape (Fig. 2.1A). This is phase I of the evaporation process. After about 20 $\mathrm{s}$, indeed microdroplets nucleate at the rim of the drop, as seen in Figure $2.2 \mathrm{~B}$ or Figure 2.3B. This signals the onset of phase II, sketched in Figure 2.4A: The microdroplets are convected throughout the whole Ouzo drop, giving it 
its 'milky' appearance (Fig. 2.1B). Due to the declining ethanol concentration, the liquid becomes oil-oversaturated (cf. Materials and Methods section and Fig. 2.7). This oil-oversaturation leads to further oil droplet growth [36] and coalescence (Fig. 2.2 C). Finally, an oil ring appears, caused by the deposition of coalesced oil microdroplets on the surface (sketch in Fig. 2.4 $\mathrm{B}$ and Figs. 2.1 C, 2.2 $\mathrm{D}$ and 2.3A). The zoomed-in graph in Figure 2.2 $\mathrm{D}$ and Figure 2.3A reveal the presence of three contact lines (CL) near the oil ring: CL-1, where mixture, surface and oil meet, CL-2, where mixture, oil and air meet, and CL-3, where oil, substrate and air meet. The drop is still opaque due to the presence of the numerous oil microdroplets in the bulk. However, after about four minutes all ethanol has evaporated. In this phase III, most of the oil droplets have coalesced to an oil ring at the rim of the drop, which now is transparent again (Figs. 2.1D, 2.2 E, and 2.3 C and sketch in Fig. 2.4 C). In this now phase-inverted phase the drop has a very characteristic non-spherical cap-shape, with a water drop sitting on an oil ring. Subsequently, the water drop evaporates more and more. The last traces of water are seen as water microdroplets in the bulk of the remaining spherical-cap shaped sessile oil drop (Fig. 2.2F, phase IV), which now again has a single contact line. After around 14 minutes of evaporation, only a tiny sessile oil droplet is left (with $1 / 70$ th of the original drop volume), now in spherical cap shape again (Fig. $2.1 \mathrm{E}$ and sketch Fig. 2.4D).

The four life phases of the evaporating Ouzo drop are not only seen visually, but also reflect in various quantitative measures of the drop geometry, as extracted from the images of Figures 2.1 and 2.2, according to the procedure described in Supporting Information and Fig. 2.9. In Figure 2.5A-D we show the measured drop volume $V(t)$, its contact diameter $L(t)$ and the diameter $L^{*}(t)$ of the water drop sitting on the oil ring, the corresponding contact angles $\theta(t)$ and $\theta^{*}(t)$, and the radius of curvature $R(t)$ of the drop. The four characteristic phases are separated by three black vertical dashed lines: Phase I, before the Ouzo effect starts, i.e. before the microdroplets are optically observed at the rim of the drop; phase II, before all ethanol in the drop has evaporated, which is determined from a force balance analysis at CL-2 as detailed in Materials and Methods section; phase III, before the water in the drop has evaporated, i.e. before $\theta(t)$ approaches the contact angle of pure anise oil; and phase IV, when the drop consists of oil only.

After approximately $60 \mathrm{~s}$, the oil ring appeared which is indicated in Figure 2.5 as a green vertical solid line. From that moment, the evolution of the two additional geometrical parameters $L^{*}$ and $\theta^{*}$ is shown. In phases I and II, $V(t)$ and $L^{(*)}(t)$ decrease very fast, due to the high evaporation rate of ethanol. Once all ethanol has evaporated, at the transition from phase II to phase 

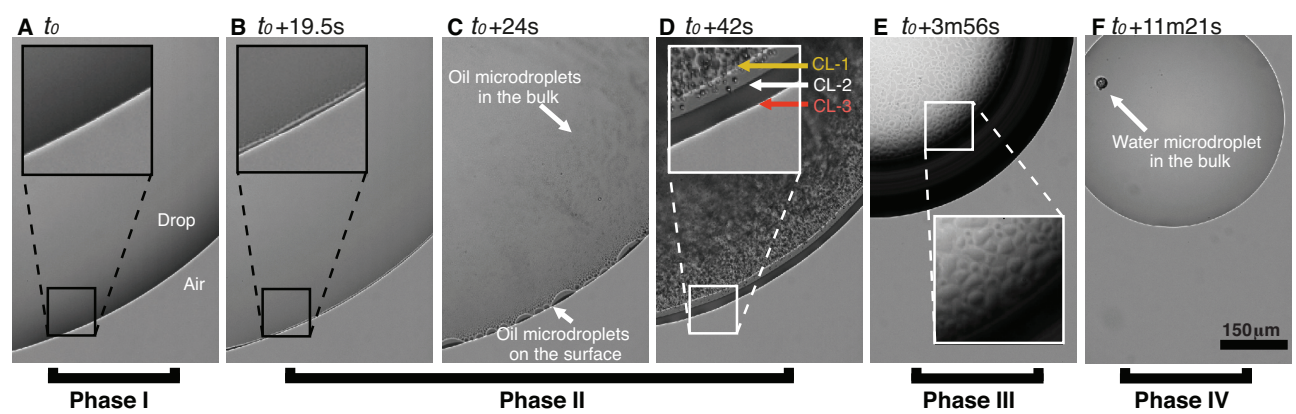

Figure 2.2: Bottom-view snapshots of the contact region of an evaporating $0.7 \mu \mathrm{L}$ Ouzo drop of the same composition as in Figure 2.1. (A), Phase I: The Ouzo drop is totally transparent with a clearly defined contact line (CL). (B) Phase II: After around $20 \mathrm{~s}$, the contact line is thickened due to the nucleation of oil microdroplets at the rim as shown in the zoomed-in graph. (C) Oil microdroplets nucleated near the contact line are convected throughout the entire drop. Meanwhile, the oil microdroplets at the contact line grow and coalesce. (D) An oil ring has appeared, caused by the deposition of coalesced oil microdroplets on the surface. The zoomed-in graph reveals the presence of three contact lines CL-1, CL-2, and CL-3 near the oil ring, as explained in the main text. The drop is opaque by the presence of numerous oil microdroplets in the bulk. (E) Phase III: The outer diameter of the oil ring is smaller, while the thickness is much larger. The drop has become transparent again and many merged oil microdroplets on the surface can be observed. (F) The drop is transparent with a single contact line CL-3. A water microdroplet has been produced as residual of the contracting line CL-2. Finally, this remaining water dissolves into the oil and disappears, leaving a homogeneous oil drop (Phase IV).

III, there is a sharp reduction in the slope of $V(t), L^{(*)}(t)$, and $R(t)$, which in phase-inverted phase III decrease more slowly due to the lower evaporation rate of water. In this regime, a force balance holding at CL-2 reaches its steady state (Fig. 2.10). In the final phase, $V(t)$ converges to the initial volume of the anise oil (zoomed-in graph in Fig. 2.5A) and $\theta(t)$ approaches the contact angle of pure anise oil (Fig. $2.5 \mathrm{C}$ ).

\subsubsection{Numerical modelling of the evaporation process and its quanti- tative understanding}

More quantitative insight is gained from numerically modelling the evaporation process of the Ouzo drop (Video S6). Our numerical model is based on an axisymmetric lubrication approximation in the spirit of the evaporating coffee-stain lubrication models of refs. [32, 33, 38, 39, but now for a multicomponent liquid. The relative mass fractions are governed by a convectiondiffusion equation, with a sink-term at the air-drop interface, reflecting evaporation, and ethanol-concentration-dependent material parameters such as density, diffusivity, viscosity, surface tension, and activity coefficients (quantifying the evaporation rate). These composition-dependent properties are depicted 

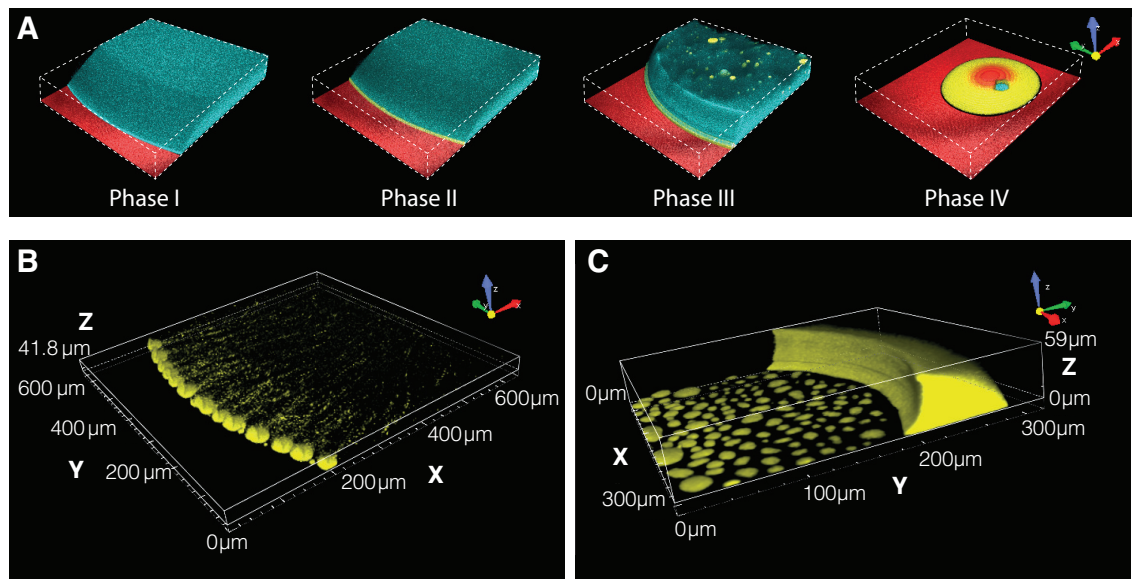

Figure 2.3: Confocal images of the Ouzo drop in different phases. Water/ethanol solution (blue) and oil (yellow) were labeled with different dyes in the confocal experiment. (A) Morphology of the evaporating Ouzo drop corresponding to four different life phases, taken from a confocal view (Video S4). The scan volume of the confocal microscope is $560 \mathrm{\mu m}$ $560 \mu \mathrm{m} 90 \mu \mathrm{m}$. (B) The coalesed oil microdroplets on the surface and fresh nucleated oil microdroplets in the bulk were presented in $3 \mathrm{D}$ at $t_{0}+26 \mathrm{~s}$ (early in phase II). For the appropriate spatial resolution the 3D images had to be taken over a period of $0.9 \mathrm{~s}$, leading to motion blur of the moving oil microdroplets. (C) As the oil ring shrinks over time, surface oil microdroplets are destined to be absorbed as shown at $t_{0}+374 \mathrm{~s}$ (early in phase III). A confocal movie of the early nucleation process is supplied as Video S5.

in Fig. 2.12. The Ouzo drop is described assuming axial symmetry, with the liquid-air interface given by the height function $h(r, t)$ and the fluid velocity $\vec{v}=(u, w)$ (cf. Fig. 2.11). Details of the model are given in Supporting Information.

The fundamental difference between the evaporation of a pure liquid 39] and that of a mixture is the vapor-liquid equilibrium. While in the case of a pure liquid $\alpha$ the vapor concentration $c_{\alpha}$ (mass per volume) directly above the liquid-air interface is saturated, i.e. $c_{\alpha}=c_{\alpha \text {,sat }}$, it is lower for the case of mixtures. The relation between liquid composition and vapor composition is expressed by Raoult's law. As in the evaporation model for a pure liquid [39], the evaporation rate $J_{\alpha}$ is obtained by solving the quasi-steady vapor-diffusion $\nabla^{2} c_{\alpha}=0$ in the gas phase with the boundary conditions given by Raoult's law above the drop, by the no-flux condition $\left.\partial_{z} c_{\alpha}\right|_{r>L / 2, z=0}=0$ at the drop-free substrate, and far away from the drop by the given vapor concentrations $c_{\alpha}=0$ for ethanol and $c_{\alpha}=c_{\alpha, \infty}=R H_{\alpha} c_{\alpha, \text { sat }}$ for water, where $R H_{\alpha}$ is the relative humidity. The relative humidity can be measured to some limited precision, but here had to be corrected for to better describe the experimental data, as detailed in Materials and Methods section. Finally, the evaporation rates are given by $J_{\alpha}=-D_{\alpha, \text { air }} \partial_{n} c_{\alpha}$ with the vapor diffusion coefficients $D_{\alpha, \text { air }}$ of $\alpha$ in 
A The beginning of phase II:
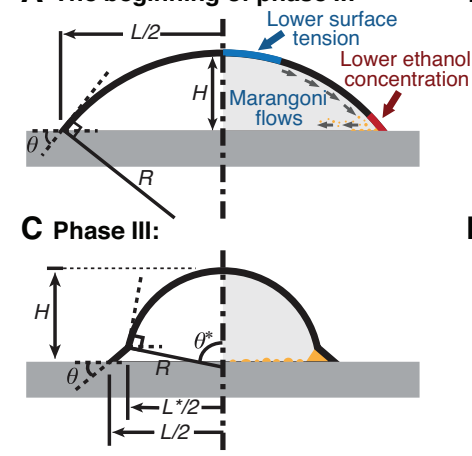

B The later regime of phase II:

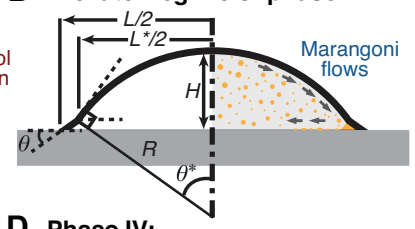

D Phase IV:

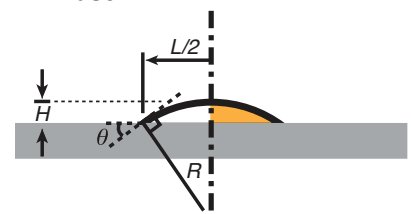

Figure 2.4: Schematics of the Ouzo drop with the definitions of the geometrical parameters at four particular moments. (A) Due to the preferential evaporation of ethanol near the contact line, the nucleation of oil microdroplets starts in this region. The surface tension gradient drives a Marangoni flow that leads to a convection of the oil microdroplets. Despite the nonuniform surface tension, the contour of the drop is well described by a spherical cap with radius $R$. (B) At later times of regime phase II, the oil microdroplets are present in the entire drop and also cover the surface. Meanwhile, the oil ring (indicated by the orange triangular region) has appeared, which allows for the definition of two new geometrical parameters $L^{*}$ and $\theta^{*}$. (C) After the ethanol content has completely evaporated, the main part of the drop consists of water only. The oil microdroplets in the bulk have coalesced and form a thicker oil ring and larger oil microdroplets on the substrate. Due to the relatively slow evaporation rate of water as compared to ethanol, this stage lasts much longer than phase II. (D) Finally, only the non-volatile oil remains after both ethanol and water have evaporated. The sessile drop now again has a spherical-cap shape.

air. In contrast to the evaporation of a pure fluid, the evaporation rate of a mixture component does not only depend on the geometric shape of the drop, but also on the entire composition along the liquid-air interface. The resulting $r$-dependent height loss due to evaporation is given in Supporting Information.

In the simulations, the fitted experimental data $\theta^{*}$ (shown in Fig. 2.5G) were used as the time-dependent contact angle. The quantitative measures of the drop geometry resulting from the numerical simulations are shown in Figures $2.5 \mathrm{E}, 2.5 \mathrm{~F}$, and $2.5 \mathrm{H}$, together with the experimental data, showing excellent quantitative agreement. From Figure 2.5E, which next to the total volume $V(t)$ also shows the partial volumes of the three components water, ethanol, and oil, we can reconfirm that the volume loss is initially mainly due to the evaporation of ethanol (phase I and II), followed by a slower evaporation of the remaining water (phase III). Finally, only the tiny non-volatile oil droplet remains (phase IV).

Our numerical simulations of the process allow us to deduce the fully spatially resolved mass fraction and velocity fields, $y_{\alpha}(r, z, t)$ and $\vec{v}(r, z, t)$, respectively. In Figures 2.6 and 2.6 $\mathrm{b}$ we show the ethanol mass fraction $y_{\mathrm{e}}(r, z, t)$ and the velocity field $\vec{v}(r, z, t)$ for two different times $t=20 \mathrm{~s}$ and $t=180 \mathrm{~s}$. It 

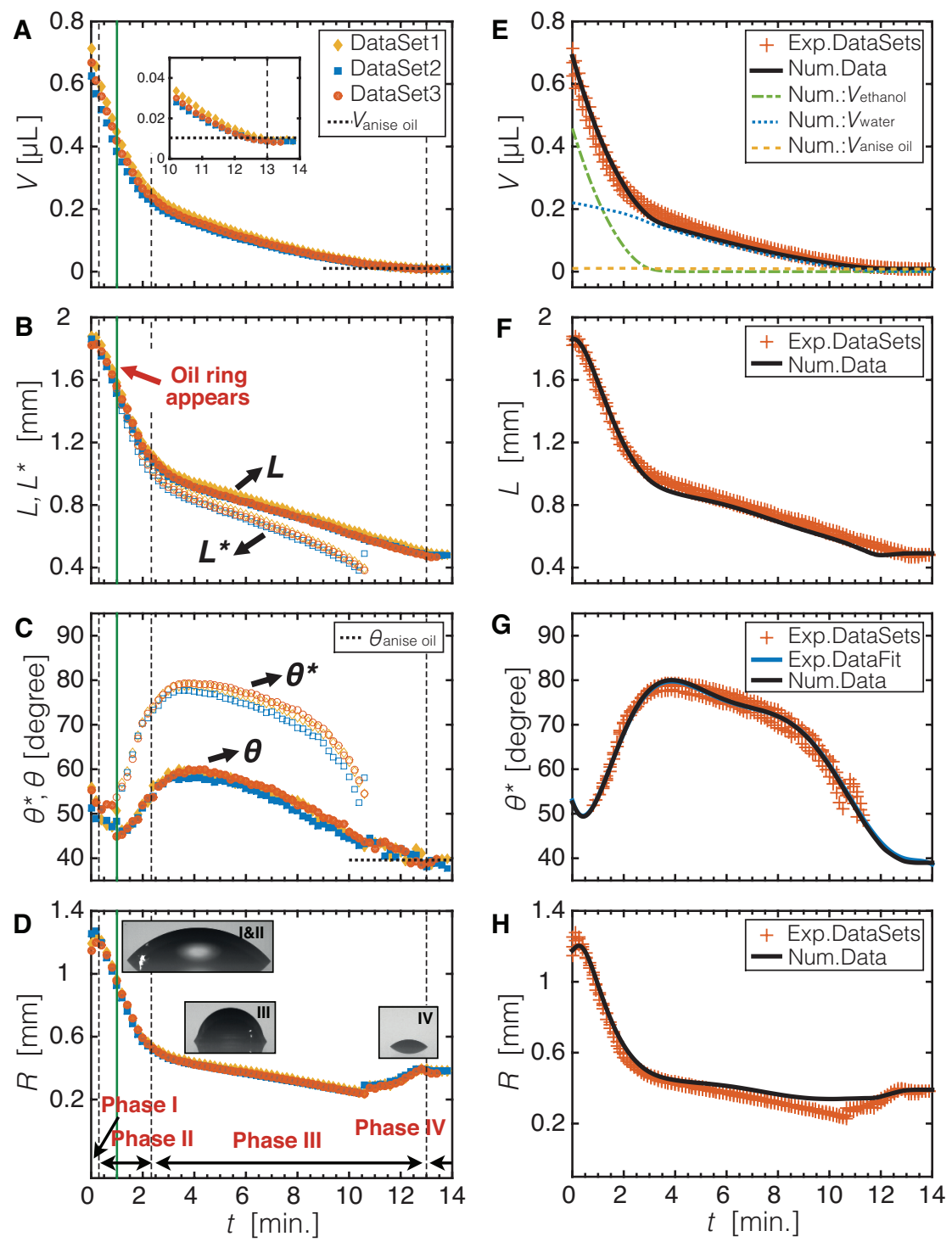

Figure 2.5: Experimental (A-D) and numerical (E-H) results for the temporal evolution of the geometrical parameters: Volume $V(\mathbf{A}, \mathbf{E})$, lateral sizes $L$ and $L^{*}(\mathbf{B}, \mathbf{F})$, contact angles $\theta$ and $\theta^{*}(\mathbf{C}, \mathbf{G})$, and radius of curvature $R(\mathbf{D}, \mathbf{H})$. The vertical dashed lines mark the transition from one phase to another.

is clearly visible how the preferential evaporation of ethanol near the contact line, which leads to a larger surface tension there, drives a fast Marangoni flow. As a consequence, ethanol is quickly replenished at the liquid-air interface and can completely evaporate. We note that the direction of the convection roll inside the drop is opposite to the case of a pure liquid, where the flow goes 


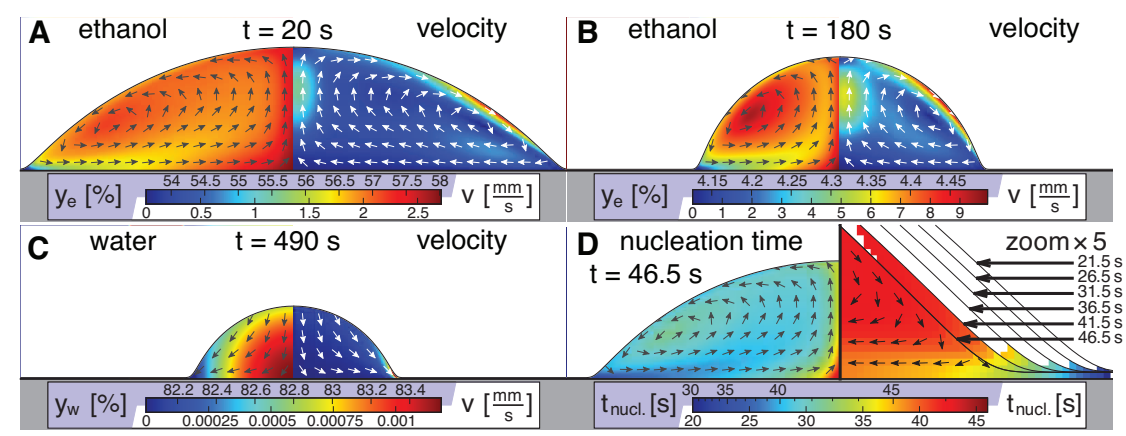

Figure 2.6: Snapshots of the numerical results at three different times $t=20 \mathrm{~s}(\mathrm{~A}), \mathrm{t}=180$ $\mathrm{s}(\mathrm{B})$ and $\mathrm{t}=490 \mathrm{~s}(\mathrm{C}) .(\mathbf{A}, \mathbf{B})$ Mass fraction of ethanol $y_{\mathrm{e}}(r, z, t)$ and fluid velocity field $\vec{v}(r, z, t)$, whose direction is indicated by the arrows and whose modulus by the color-code. At the later time $\mathrm{t}=490 \mathrm{~s}$ in $(\mathbf{C})$, the water concentration is plotted instead of the ethanol concentration (which then is close to zero), again together with the velocity field. (D) Oil droplet nucleation time $t_{\text {nucl. }}$. The right side shows a zoom-in of the region around the rim. A movie of the numerical simulation is supplied as Video S6.

outwards at the bottom of the drop and inwards at the liquid-gas interface [33, 38, 39. We also note that the ethanol concentration differences are relatively small - in the beginning about $3 \%$ and later not more than $0.5 \%$ - but nonetheless sufficient to drive a strong Marangoni flow with velocities up to the order of $10 \mathrm{~mm} / \mathrm{s}$. Due to the high contact angle during phases II and III, the lubrication approximation predicts the precise values of the velocity only to a limited accuracy. The qualitative flow field and the order of magnitude, however, have been validated by a comparison with the corresponding non-approximated Stokes flow at individual time steps. Figure 2.6 $\mathrm{C}$ shows the water mass fraction $y_{\mathrm{w}}(r, z, t)$ for $t=46.5 \mathrm{~s}$, since at these later times ethanol is virtually not present anymore, again together with the velocity field, which is now again outwards directly above the substrate.

Finally, in Figure 2.6D we show the oil droplet nucleation time $t_{\text {nucl. }}$, which is defined as the moment when the local composition crosses the phase separation curve and enters the Ouzo region (see Fig. 2.7A). According to the numerical results, the oil droplet nucleation starts at $20 \mathrm{~s}$ near the contact line, in perfect agreement with our experimental findings, and nucleation is possible in the entire droplet at $t=46.5 \mathrm{~s}$. 
Table 2.1: Data of the ternary diagram of ethanol-anise-water.

\begin{tabular}{|c|c|c|c|c|c|c|}
\hline \multirow{2}{*}{$\begin{array}{l}\text { Titration }^{1} \\
\text { No. }\end{array}$} & \multicolumn{2}{|c|}{ Titrant (ethanol-oil mixture) } & \multirow{2}{*}{$\begin{array}{c}\text { Titrate }^{2} \\
\text { Water }(\mathrm{ml})\end{array}$} & \multicolumn{3}{|c|}{ Weight ratios ${ }^{3}$} \\
\hline & Ethanol $(\mathrm{ml})$ & $\begin{array}{c}\text { Anise } \\
\text { oil }(\mathrm{ml})\end{array}$ & & $y_{\mathrm{w}}(\%)$ & $y_{\mathrm{e}}(\%)$ & $y_{\mathrm{a}}(\%)$ \\
\hline 1 & 0 & 0.001 & 6 & 99.98 & 0 & 0.02 \\
\hline $2(\mathrm{a})^{4}$ & 1 & 0.001 & 2.7724 & 73.47 & 26.50 & 0.03 \\
\hline $3(\mathrm{~b})$ & 1 & 0.002 & 2.2186 & 68.89 & 31.05 & 0.06 \\
\hline 4 & 1 & 0.01 & 1.0658 & 51.34 & 48.17 & 0.48 \\
\hline $5(\mathrm{c})$ & 1.2 & 0.02 & 1.1491 & 48.50 & 50.65 & 0.84 \\
\hline 6 & 1 & 0.03 & 0.7671 & 42.69 & 55.56 & 1.67 \\
\hline $7(\mathrm{~d})$ & 1 & 0.04 & 0.6785 & 39.48 & 58.19 & 2.33 \\
\hline 8 & 1 & 0.05 & 0.5821 & 35.67 & 61.27 & 3.06 \\
\hline $9(\mathrm{e})$ & 1.7 & 0.1 & 0.9211 & 33.85 & 62.47 & 3.67 \\
\hline 10 & 1.5 & 0.1 & 0.7154 & 30.90 & 64.78 & 4.32 \\
\hline $11(\mathrm{f})$ & 1.2 & 0.1 & 0.5014 & 27.83 & 66.61 & 5.55 \\
\hline $12(\mathrm{~g})$ & 0.7 & 0.1 & 0.2261 & 22.03 & 68.22 & 9.75 \\
\hline $13(\mathrm{~h})$ & 1 & 0.2 & 0.2563 & 17.60 & 68.67 & 13.73 \\
\hline $14(\mathrm{i})$ & 0.8 & 0.2 & 0.1727 & 14.73 & 68.22 & 17.05 \\
\hline 15 & 0.7 & 0.3 & 0.1173 & 10.50 & 62.65 & 26.85 \\
\hline 16 & 0.6 & 0.4 & 0.0842 & 7.77 & 55.34 & 36.89 \\
\hline 17 & 0.5 & 0.5 & 0.0635 & 5.97 & 47.01 & 47.01 \\
\hline 18 & 0.4 & 0.6 & 0.0476 & 4.54 & 38.18 & 57.27 \\
\hline 19 & 0.3 & 0.7 & 0.0404 & 3.88 & 28.84 & 67.28 \\
\hline 20 & 0.2 & 0.8 & 0.0351 & 3.39 & 19.32 & 77.29 \\
\hline 21 & 0 & 1 & 0.0041 & 0.41 & 0 & 99.59 \\
\hline
\end{tabular}

${ }^{1}$ The titration was conducted at a temperature of around $22^{\circ} \mathrm{C}$.

${ }^{2}$ Aliquot was $0.0015 \mathrm{ml}$, which was the minimum volume of the water droplet created by the pipette needle during titration.

${ }^{3}$ Density of anise oil at $22{ }^{\circ} \mathrm{C}$ was measured as $0.989 \mathrm{~g} \mathrm{ml}^{-1}$. Water and ethanol density at $22^{\circ} \mathrm{C}$ was obtained from a handbook [64 by linear interpolation.

${ }^{4}$ Corresponding to the labels in the ternary diagram (cf. Fig. 2.7A). 


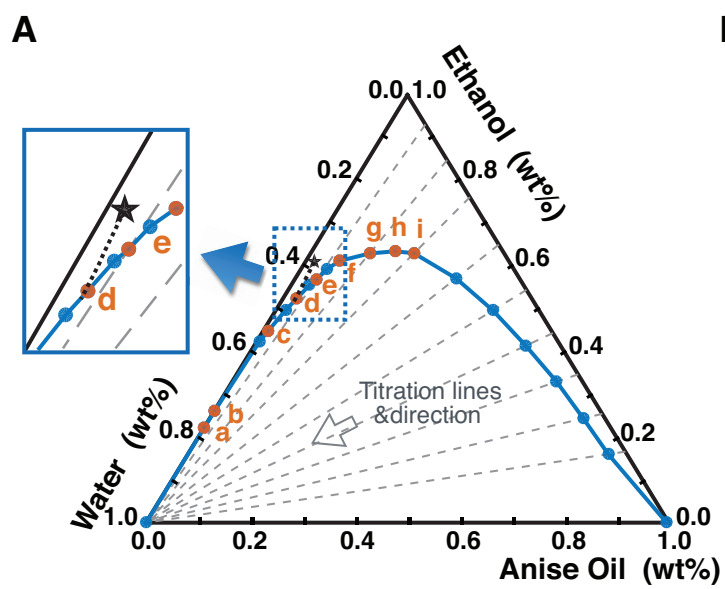

B Shake them well with a vortex mixer:

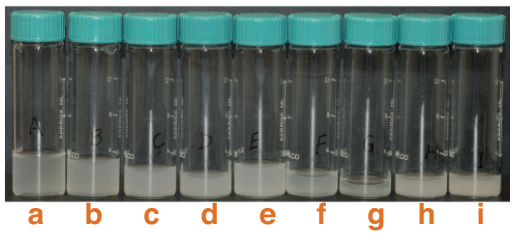

15 min later after shaking well:

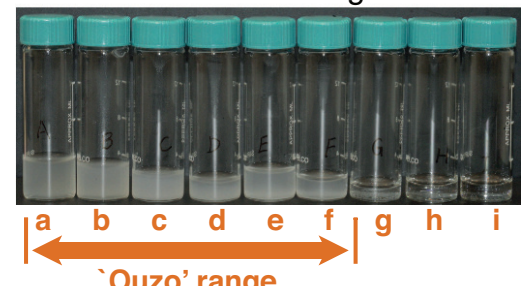

Figure 2.7: (A) shows the ternary diagram of water, ethanol and anise oil. The blue solid line is the measured phase separation curve. The black star and the black dotted line in the magnified figure indicate the initial composition of the Ouzo drop and its path in time according to the numerical simulation. The gray dashed lines show paths of some composition coordinates from the titration experiments; (B) The stability of the macrosuspension for the compositions a-i in the ternary graph were compared. The comparison reveals that the curve along the dots a to $\mathrm{f}$ is the boundary of the Ouzo region, i.e. the critical composition at which the Ouzo effect sets in.

\subsection{Methods}

\subsubsection{Ternary diagram and initial composition of the Ouzo drop}

The ternary liquid of the Ouzo drop in this study was the mixture of Milli-Q water (produced by a Reference A+ system, Merck Millipore, at $18.2 \mathrm{M} \Omega \mathrm{cm}$ ), ethanol (EMD Millipore, Ethanol absolute for analysis) and anise oil (Aldrich, Anise oil). The ternary diagram of the mixture was titrated at a temperature of $22{ }^{\circ} \mathrm{C}$, which is similar to the environmental temperature during the evaporation experiment. 21 groups of ethanol and anise oil mixtures with different component weight ratios were properly prepared to be used as titrants (see Table 2.1). The volume of water (titrate) was precisely measured by a motorised syringe pump (Harvard, PHD 2000). For each ethanol and anise oil mixture, a phase-separation point was determined as shown in Fig. 2.7A. Photographs of the macrosuspensions corresponding to the different phase-separation points were taken. Thereby, the stability of the macrosuspension along the phase separation curve was determined (Fig. 2.7B). Starting with point g, the homogeneous macrosuspension is not stable anymore. The part of the curve with a stable macrosuspension was identified as the boundary of the Ouzo region in the ternary diagram, which is labeled Ouzo range. According to the ternary 
diagram, the initial composition of the Ouzo drop was chosen as $37.24 \%$ water, $61.06 \%$ ethanol and $1.70 \%$ anise oil in terms of weight fractions, which is indicated by the black star in Fig. 2.7A. Starting from this initial point, the drop composition is guaranteed to cross the phase separation curve and enter the Ouzo region during the evaporation process. A black dotted line in the magnified subfigure of Fig. 2.7A shows the numerically obtained temporal evolution of the composition near the contact line of the Ouzo drop.

\subsubsection{Experimental setups}

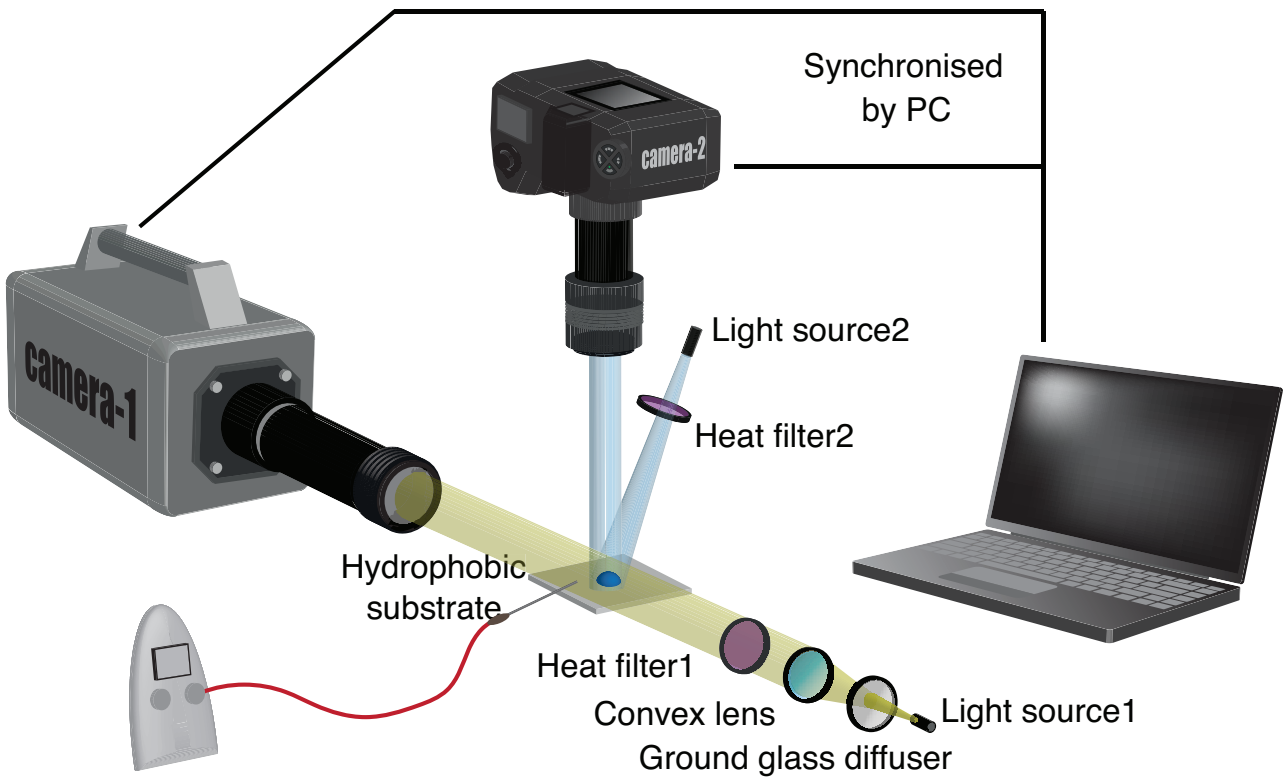

Thermo hygrometer

Figure 2.8: Experimental setup showing the evaporation of an Ouzo drop being recorded by two synchronised cameras. A fine needle (not shown here) was used to produce and place the drop on the hydrophobic substrate and then gently moved far away from the experimental region. Heat filter1, a convex lens and a ground glass diffuser were placed in front of light source1 (Schott ACE I) to create a collimated light beam without infrared light. Another heat filter was inserted in the light path of light source 2 (Olympus ILP-1). The ambient temperature and the relative humidity were determined by a thermo-hygrometer.

A $0.7 \mu \mathrm{L}$ Ouzo drop $(37.24 \%$ water, $61.06 \%$ ethanol and $1.70 \%$ anise oil in terms of weight fractions) was produced through a custom needle (Hamilton, O.D. $\times$ I.D. $(\mathrm{mm}): 0.21 \times 0.11)$ by a motorised syringe pump (Harvard, PHD 2000). The whole evolution of the Ouzo drop was observed by two synchronised cameras, one (Photron Fastcam SA-X2 64GB, 50 fps at 1,024 × 1,024 pixel resolution) affixed with a high-magnification zoom lens system (Thor- 
labs, MVL12X3Z) for side-view recordings and another (Nikon D800E, 25fps at 1,920 $\times 1,080$ pixel resolution) affixed with an identical lens system for top-view recordings (Figure 2.1). The temperature around the evaporating drop was measured using a thermometer sensor. The relative humidity in the lab was measured with a standard hygrometer $( \pm 3 \% \mathrm{RH}$ for $35 \% \sim 70 \% \mathrm{RH}$ at $20^{\circ} \mathrm{C}$ ) The temperature of the three experimental datasets in Figure 2.5 was between $21^{\circ} \mathrm{C}$ and $22.5^{\circ} \mathrm{C}$. The relative humidity was around $40 \%$. The image analysis was performed by custom-made MATLAB codes. In order to have a detailed observation of the evolutionary process at the rim of the Ouzo drop, an inverted microscope (Olympus GX51) was used to focus on the contact region. A fast speed camera (Photron Fastcam SA-X2 64GB, 50 fps at 1,024 $\times$ 1,024 pixel resolution) was connected to the microscope with an intermediate tube. Figure 2.2 was taken with a $20 \times$ long working m-plan fluorite objective (Olympus MPLFLN20XBD, Wd $=3.0 \mathrm{~mm}, \mathrm{NA}=0.45$ ). Besides 2D imaging, we also took advantage of a confocal microscope (Nikon Confocal Microscopes A1 system) in stereo-imaging. A real-time observation was carried out to monitor the movement of the oil droplets due to the convective flow and the formation of oil ring in a $3 \mathrm{D}$ view. A $20 \times$ air objective (CFI Plan Apochromat $\mathrm{VC} 20 \times / 0.75 \mathrm{DIC}, \mathrm{NA}=0.75, \mathrm{WD}=1.0 \mathrm{~mm})$ and a $40 \times$ air objective (CFI Plan Fluor $40 \times / 0.75 \mathrm{DIC}, \mathrm{NA}=0.75, \mathrm{WD}=0.66 \mathrm{~mm}$ ) were employed to take Figures 2.3ABC, respectively. In Figures $2.3 \mathrm{~B}$ and $\mathrm{C}$, anise oil was labeled by Nile Red (Microscopy grade, Sigma-Aldrich, Netherlands). In Figure 2.3A, in order to simultaneously label oil and solution with different color dyes during the whole evaporating process, anise oil was replaced by trans-Anethole oil (99\%, Sigma-Aldrich, Netherlands) labeled by perylene (sublimed grade, $\geq 99.5 \%$, Sigma-Aldrich, Netherlands) in yellow color. Water/ethanol mixture was labeled by fluorescein 5(6)-isothiocyanate (High performance liquid chromatography, Sigma-Aldrich, Netherlands) in blue color.

\subsubsection{Image analysis and data calculation}

The image analysis was performed by custom-made MATLAB codes, through which all the geometric parameters at every frame were successfully determined, such as drop volume $V$, contact angles $\theta$ and $\theta^{*}$, lateral sizes $L$ and $L^{*}$ and droplet height $H$ (cf. Fig. 2.9A). The drop volume was calculated by adding the volumes of horizontal disk layers, assuming rotational symmetry of each layer with respect to the vertical axis. The contact angle $\theta$, between the blue and green lines in Fig. 2.9A, was estimated from the profile at the contact region by polynomial fits, while $\theta^{*}$, between the red and yellow lines, was calculated by a spherical cap approximation (purple circle). The drop contour above the oil ring was also fitted by elliptical fits. Since the drop size 
is smaller than the capillary length $\kappa^{-1}=\sqrt{\frac{\gamma}{\rho g}}(2.7 \mathrm{~mm}$ for water, $1.7 \mathrm{~mm}$ for ethanol), the ellipticity of the top cap, defined as the ratio between the difference of the two semi-axes and the radius, was always below $10 \%$ during phase II (Fig. 2.9B). After around 11 minutes, both the spherical cap approximation and the elliptical fittings for the water contour above the oil ring were not sufficiently accurate. The water drop diameter $L^{*}$ was too small (less than $0.4 \mathrm{~mm}$ ) and there were not enough pixels to perform the contour fits. Therefore, we stopped calculating $\theta^{*}$ from a spherical cap approximation at around 11 minutes, when the ellipticity exceeds $10 \%$.

A

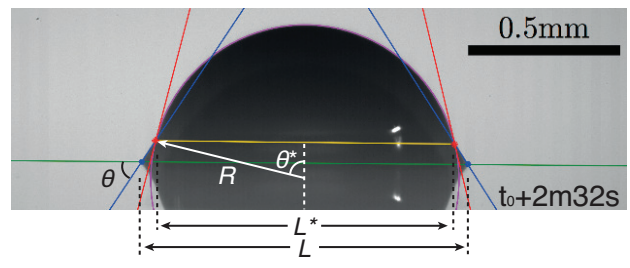

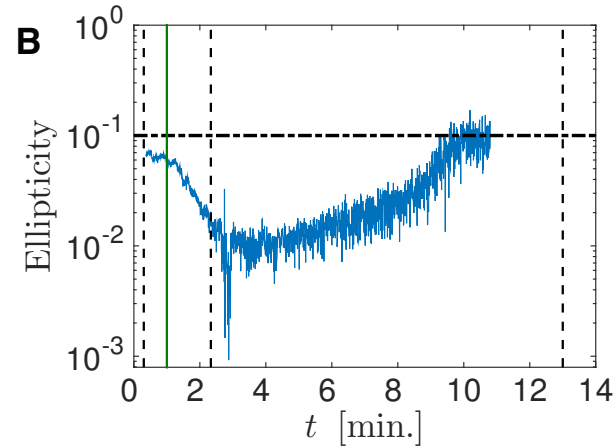

Figure 2.9: Details of the experimental image analysis: (A) A representative raw image displayed with the corresponding results of the image analysis. $\theta$ was estimated by a polynomial fitting; $\theta^{*}$ was calculated by a spherical cap approximation. (B) The ellipticity, defined as the ratio between the difference between the lengths of the two semi-axes and the radius, is depicted. $\theta^{*}$ was only calculated for spherical cap approximations with ellipticities less than $10 \%$. Three black vertical lines are four phases separation moments. Green vertical line indicates the appearance of the oil ring.

\subsubsection{Definitions of the four life phases of an evaporating Ouzo drop}

We divided the Ouzo drop evaporation process into four phases: Phase I is defined as the initial regime, before the critical phase separation composition is attained at the contact line. Phase II is the time from the initial occurrence of the oil nucleation until the complete evaporation of the ethanol component. Phase III is the regime when the remaining water amount in the drop evaporates. The final phase IV is the period after the the remaining water has evaporated. The first black vertical dashed lines (separation between phases I and II) and the third one (separation of phases III and IV) in Figure 2.5 were able to be optically determined from the top or bottom view video recordings. However, the transition between phase II and phase III cannot be detected from the video recordings. Instead, the second black vertical dashed line in 
Figure 2.5 was determined from an equilibrium analysis as a simplified model (cf. Fig. 2.10A): at the air-mixture-oil contact line (CL-2 in Figs. 2.2B, $2.3 \mathrm{~A}$ and $2.3 \mathrm{C}$ ), a force balance holds. The influence of the line tension on the balance can be neglected [65]. Each variation of the composition in the drop alters the equilibrium of this balance 65, 66]. At the moment when ethanol has completely evaporated, this equilibrium attains its steady state. From that moment, the three phases which meet at the contact line CL-2 are water from the liquid of the drop, anise oil from the oil ring and air from the surroundings. The composition of the air phase near the contact line CL-2 is assumed to be constant. Hence, the angle between the mixture-air interface and the oil-air interface has to be constant. Mathematically speaking, this means that $\Delta \theta$ has to be a constant. The quantity $\Delta \theta$ was estimated by the subtraction $\theta^{*}-\theta$, since the dimension of the oil-air interface is small in the initial part of phase III. In Fig. $2.10 \mathrm{~B}$, the evolution of $\Delta \theta$ as a function of time is shown. It is clearly visible that after a rapid increase $\Delta \theta$ remains constant for a very long time. Therefore, we fitted $\Delta \theta$ from time $t_{a}$ to time $t_{z}=480 \mathrm{~s}$ by a constant $c$. The inserted graph in fig. $2.10 \mathrm{~B}$ shows the relation between $c\left(t_{a}\right)$ and $t_{a}$. We selected the time $t_{a}=140 \mathrm{~s}$ as the separation moment between phase II and phase III.

A

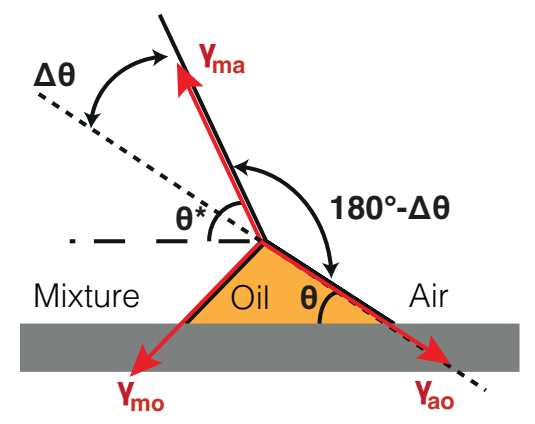

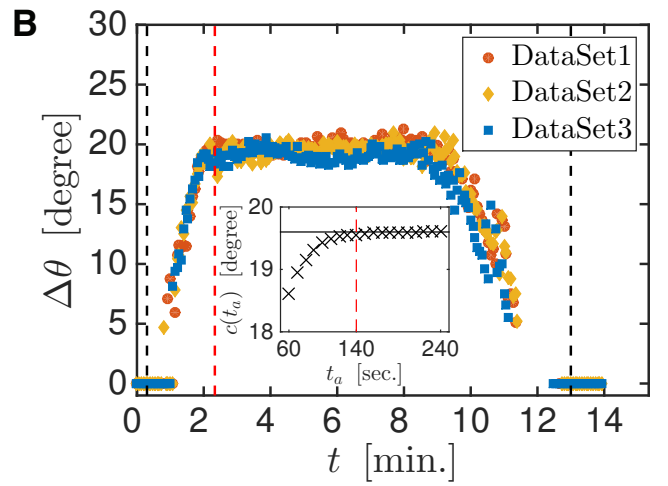

Figure 2.10: (A) Cross-sectional sketch of the oil ring and the equilibrium of the air-mixtureoil contact line. When the mixture predominantly consists of water, the equilibrium is steady and $\Delta \theta$ is constant. (B) shows experimental data of the temporal evolution of $\Delta \theta$. The red vertical dashed line is the separation moment between phases II and III. It is defined as the moment when $\Delta \theta$ starts to be constant. The inserted figure depicts the value $c\left(t_{a}\right)$ fitted over the range $\left(t_{a}, 480 \mathrm{~s}\right)$ with a constant $c$. 


\subsubsection{Numerical model}

The evolution of the drop shape $h(r, t)$ (cf. Fig. 2.11) is solved by a diagonallyimplicit Runge-Kutta method, the vapor diffusion-limited evaporation rates are calculated by a boundary element method and the convection-diffusion equations for the composition are treated with an upwind finite differences scheme. For the composition-dependency of the mass density, the surface tension, the diffusion coefficient, the viscosity and the activity coefficients, we fitted experimental data of water-ethanol mixtures or used appropriate models (cf. Fig. 2.12). Details can be found in the Supporting Information and in supplementary material. Our model was validated for the case of pure water by comparison with the experimental data of Gelderblom et al. [33.

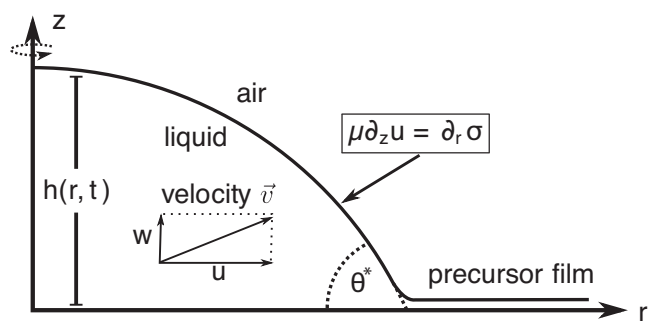

Figure 2.11: Schematic illustration of the model. The shape $h(r, t)$ of the drop is described in axisymmetric cylinder coordinates. Due to different volatilities of the components, a surface tension gradient is induced that drives a Marangoni flow. The moving contact line with contact angle $\theta^{*}$ is realised by a precursor film.

\subsubsection{Determination of the relative humidity}

For the numerical simulation, we have assumed a temperature of $T=21{ }^{\circ} \mathrm{C}$ and a relative humidity of $R H_{\mathrm{e}}=0$ for ethanol. Since the experimental determination of the relative humidity $R H_{\mathrm{w}}$ of water is error-prone, we have determined it as follows: At the beginning of phase III, the drop consists almost entirely of water, since the ethanol content has already evaporated and the amount of oil is still small in comparison to the remaining water volume. Therefore, we used our numerical model to fit $R H_{\mathrm{w}}$ based on the experimental data for the volume evolution $V(t)$ during the time from $t=140 \mathrm{~s}$ to $t=300 \mathrm{~s}$. The resulting water humidity reads $R H_{\mathrm{w}}=63 \%$.

\subsection{Conclusions}

In summary, we have experimentally and numerically studied the evaporation of a millimetre sized sessile Ouzo drop on a hydrophobic substrate. How stim- 

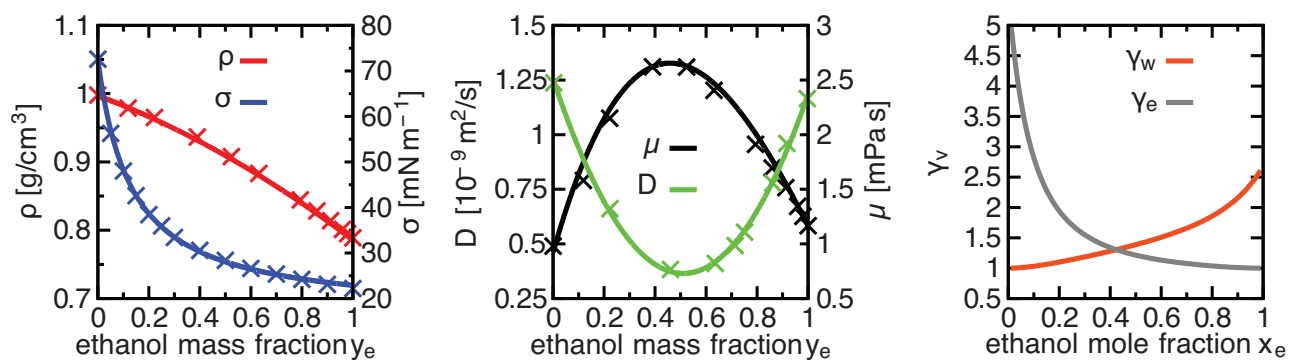

Figure 2.12: Composition-dependence of the physical liquid properties: We have fitted the following experimental data of water-ethanol mixtures for the incorporation into our model: the mass density $\rho$ and the viscosity $\mu$ [67, the surface tension $\sigma$ 68] and the mutual diffusion coefficient $D$ [69]. The activity coefficients $\gamma_{\alpha}$ for the evaporation rate were calculated by AIOMFAC [70, 71] (http://www.aiomfac.caltech.edu).

ulating it can be to study the evaporation of alcoholic drinks has interestingly also been shown in a very recent parallel but independent work by Kim et al. [72, who studied the drying of whisky droplets, which give a uniform deposition pattern. For that system suspended material and surface-absorbed macromoleculars play a major role and offer a physicochemical avenue for the control of coatings. From our point of view, just as the evaporating whisky droplet, also the evaporating ouzo droplet can advance our scientific understanding of complex flow phenomena and phase transitions and their interaction. In this paper we have observed evaporation-triggered phase transitions and the nucleation of oil microdroplets, first at the edge of the Ouzo drop and then allover, followed by a phase inversion, and altogether four different life phases of the ouzo drop, which serves as paradigmatic model system for ternary mixtures of liquids with different volatilities and mutual solubilities. Here, water as the second but most volatile liquid (after the very quickly evaporating ethanol) also evaporates in about ten minutes, leaving behind a tiny drop of anise oil. For other ternary mixtures only one liquid may be volatile, implying phase III with a binary mixture and nucleated microdroplets of one liquid and its peculiar optical properties would be the final state.

Tuning and optimizing the material and chemical properties of the individual liquids in the ternary mixture such as volatilities and mutual solubilities and polymerisibility (e.g. under UV exposure such as in ref. [73]) offers a plethora of applications for medical diagnostics, the controlled deposition of complex liquids in the food and cosmetic industry and for coating applications [74-78, in agriculture, or the food or cosmetics industry, for inkjet printing of LED or OLED devices and solar cells [1,4, 79], and for rapid manufacturing. Here we studied the deposition on smooth surfaces, but pre-patterning the surface with hydrophobic patches [80] offers even further opportunities, 
by directing the nucleation of nano- or microdroplets at will, allowing for the self-organised bottom-up construction of structures. 


\section{3 \\ Evaporating spherical ouzo drops ${ }^{m}$ 闰}

Evaporation of multi-component drops is crucial to various technologies and has numerous potential applications because of its ubiquity in nature. Superamphiphobic surfaces, which are both superhydrophobic and superoleophobic, can give a low wettability not only for water drops but also for oil drops. In this paper, we experimentally, numerically and theoretically investigate the evaporation process of millimetric sessile ouzo drops (a transparent mixture of water, ethanol, and trans-anethole) with low wettability on a superamphiphobic surface. The evaporation-triggered Ouzo effect, i.e. the spontaneous emulsification of oil microdroplets below a specific ethanol concentration, preferentially occurs at the apex of the drop due to the evaporation flux distribution and volatility difference between water and ethanol. This observation is also reproduced by numerical simulations. The volume decrease of the ouzo drop is characterized by two distinct slopes. The initial steep slope is dominantly caused by the evaporation of ethanol, followed by the slower evaporation of water. At later stages, thanks to Marangoni forces the oil wraps around the drop and an oil shell forms. We propose an approximate diffusion model for the drying characteristics, which predicts the evaporation of the drops in agreement with experiment and numerical simulation results. This work provides an advanced understanding of the evaporation process of ouzo (multi-component)

${ }^{*}$ Based on: H. Tan, C. Diddens, M. Versluis, H.J. Butt, D. Lohse, and X. Zhang, Selfwrapping of an Ouzo drop induced by evaporation on a superamphiphobic surface, Soft Matter 13(15), 2749-2759 (2017).

${ }^{\dagger}$ Numerical work performed by C. Diddens. 
drops.

\subsection{Introduction}

Drop evaporation is an omnipresent phenomenon in daily life. During this process, the liquid at the drop surface changes its phase and escapes as vapor into the ambient air. Dating back to Maxwell, the evaporation process of a drop in an ambient gas has been explored and considered mainly as a diffusioncontrolled process [81. The study of the evaporation process of sessile drops is important because of its crucial role in numerous technologies and applications, such as inkjet printing, coatings, patternings, deposition of materials, or DNA mapping [1, 7, 32, 34, 35, 38, 50, 82, 89]. In the last two decades, numerous studies have focused on understanding the evaporation process of sessile drops on solid substrates experimentally, numerically and theoretically [35, 88]. Surface properties [90 93], thermal effects [94, 95], dispersed particles in the liquid [38, 96], surfactants at the liquid-gas interface [97 99], and the liquid composition [56, 57, 59] were all found to have a contribution to the drop evaporation characteristics.

The evaporation of multi-component drops draws special interest because of its ubiquity in practice. The physicochemical properties of the drop solution dramatically enrich the system and give rise to an unexpected outcome: the different volatilities of the components lead to distinct evaporation stages with different evaporation rates and various types of wetting behavior [56, 57]. In a hydrodynamic context, flow transitions inside an evaporating binary mixture drop have been revealed, which are a result of the intense and complicated coupling of flow and the spatio-temporal concentration field [59, 100]. By controlling these mechanisms, binary drop evaporation can offer a new physicochemical way for surface coatings [72].

Recently, we used an ouzo drop as a model for a ternary liquid mixture and investigated its evaporation process on a hydrophobic surface [101]. The Greek drink Ouzo (or the French Pastis or the Turkish Raki) is a miscible solution and primarily consists of water, ethanol and anise oil. When the water concentration is increased by adding water or by reducing ethanol, the solution becomes opaque due to the "ouzo effect", i.e. the spontaneous nucleation of oil microdroplets [41-43]. We discovered how the preferential evaporation of ethanol triggers the "ouzo effect" in an evaporating ouzo drop. Four life phases can be distinguished during its drying. As a remarkable phenomenon, we found that the evaporation-triggered nucleation starts at the rim of the ouzo drops. It can be attributed to the drop geometry, namely the singularity at the rim. On the hydrophobic substrate, the ouzo drops maintain a contact 
angle $\theta$ smaller than $90^{\circ}$ (flat droplets) because of the low surface energy of ethanol in the ouzo solution. The singularity of the evaporation rate at the contact line of flat drops [32, 102] and the higher volatility of ethanol induce a maximum in the local water concentration and thereby trigger the onset of the "ouzo effect" at this position. At a later stage, the microdroplets coalesce and form an oil ring at the rim of the drop, with a water drop sitting on it. Once also the water has evaporated, only an oil drop remains.

Based on the diffusion model by Popov [32], changing the geometric configuration is a simple and direct way to change the evaporation profile and hence to induce a different concentration distribution along the liquid-air interface. In particular, a maximum evaporation rate should be found at the top of the drop when the drop has a large contact angle $\left(\theta>90^{\circ}\right)$. Thus, ouzo drops on substrates with low wettability should have the highest water concentration at the top of the drop rather than at the rim, and therefore the onset of nucleation should take place right there. However, a comprehensive numerical model by Pan et al., who took into account the evaporative cooling effect and the buoyancy-driven convective flow in the drop and vapor domains, shows that the maximum evaporation rate of drops with low wettability is still located at the contact line due to temperature effects [95]. Both of these models are only applicable to single-component drops, but hitherto it little is known about the evaporation process of multi-component sessile drops with low wettability $\left(\theta>90^{\circ}\right)$. Here we explore where the evaporationtriggered nucleation process starts for evaporating ouzo drops with $\theta>90^{\circ}$, and find out the evaporation dynamics.

First, we present an investigation of the evaporation characteristics of millimetric ouzo drops on a flat surface with a large contact angle. We performed evaporation experiments on a superamphiphobic surface, which is both superhydrophobic and superoleophobic, to achieve low wettability for the ouzo drops $\left(\theta>150^{\circ}\right.$ to start). We found that the "ouzo effect" induced by evaporation indeed preferentially takes place at the top of the drop, and two distinct stages with different evaporation rates exist. Moreover, a new remarkable phenomenon appears: part of the nucleated oil microdroplets form an oil shell wrapping up the ouzo drop, instead of forming a persistent oil ring at the contact line. Then a numerical model based on a finite element method presents additional insight into the process. Finally, we propose an approximate diffusion model for the evaporation characteristics of ouzo (multi-component) drops, and furthermore highlight and discuss the influences of Marangoni flow and the evaporative cooling effect. In summary, we provide a quantitive understanding of the evaporation process of ouzo drops experimentally, numerically and theoretically. 


\subsection{Materials and methods}

\subsubsection{Ouzo Droplet Solution and Superamphiphobic Substrate}

The ouzo drop solution was prepared with an initial composition of $32.02 \%$ (vol/vol) Milli-Q water [produced by a Reference A+ system (Merck Millipore) at $18.2 \mathrm{M} \Omega \cdot \mathrm{cm}\left(\right.$ at $\left.\left.25^{\circ} \mathrm{C}\right)\right], 66.5 \%$ ( $\mathrm{vol} / \mathrm{vol}$ ) ethanol (SIGMA-ALDRICH; $\geq$ $99.8 \%$ ) and $1.48 \%$ (vol/vol) trans-anethole (SIGMA-ALDRICH; $99 \%$ ). In this work, we used pure trans-anethole, which is the main component of anise oil, as the oil phase in ouzo drop to rule out any influence from the other components in anise oil. Experiments were carried out on a soot-templated superamphiphobic glass substrate [103, 104]. These soot-templates surfaces are formed by collecting a fractal-like network of self-assembled nearly spherical carbon particles (diameter about $40 \mathrm{~nm}$ ). The network is roughly $30 \mu \mathrm{m}$ thick and homogeneous on length scales above $5 \mu \mathrm{m}$. The soot particles are loosely connected by van der Waals forces. The network was stabilized by depositing roughly $30 \mathrm{~nm}$ of $\mathrm{SiO} 2$ using chemical vapor deposition (CVD) of tetraethyl orthosilicate (TEOS) for $24 \mathrm{~h}$. The final porosity was $90 \%$ [104]. The soot-templated superamphiphobic surface is optically transparent, so that the bottom side of the ouzo drop can be imaged while it evaporates. The static contact angles of Milli-Q water and trans-anethole oil on the substrate are $160^{\circ}$ $\pm 1^{\circ}$ and $157^{\circ} \pm 0.5^{\circ}$, respectively. Thus the ouzo drop with more than $60 \%$ ethanol can still initially hold $\sim 150^{\circ}$ static contact angle.

\subsubsection{Experimental Setup}

The evaporation experiments were performed in an empty room without any person in the room during data recording. In this sense, the entire lab can be considered as closed cell. An ouzo drop was deposited on a superamphiphobic surface through a teflonized needle (HAMILTON; 8646-02) by a motorized syringe pump (HARVARD; PHD 2000). Experiment recording started when the needle departed from the drop (defined as $t_{0}$ ). In practice there was a time delay $(\sim 22 \mathrm{~s}$ ) between starting to pump liquid out of the needle and taking the first snapshot of the drop $\left(t_{0}\right)$, leading to premature evaporation. The time delay was caused by the difficulty of depositing a sessile ouzo drop on a superamphiphobic surface. Therefore, the initial ratios of ethanol and water of the recording data should be corrected. The correction for the ethanol composition can be estimated by extrapolating the ethanol composition of the prepared solution $(66.5 \% \mathrm{vol} / \mathrm{vol})$ with respect to the delayed time and the initial volume loss rate (ref to Fig. 3.5A). With this method, a $-7.4 \%$ correction is applied to the initial ethanol composition ( $7.4 \%$ to water) for the data used in sections 3.4 and 3.5 . The entire evaporation process of the ouzo drop 

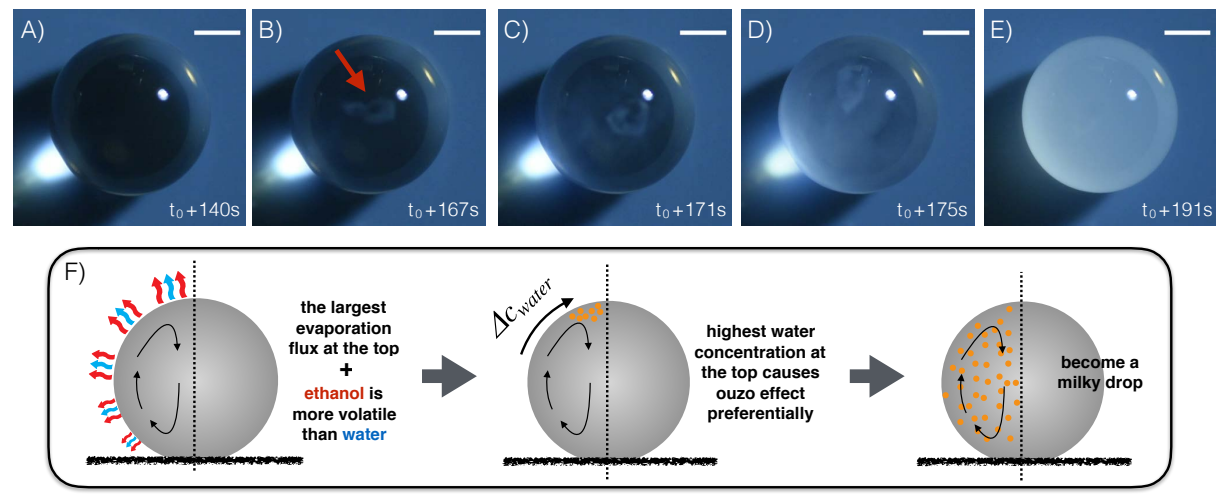

Figure 3.1: A-E) Experimental top view snapshots during the evaporation-triggered nucleation at the top of an evaporating ouzo drop on a flat superamphiphobic surface. The scale bar is $0.5 \mathrm{~mm}$. Originally, the ouzo drop is transparent in A. The light point is the focal point. At some point later in B, a region of cloudy white emulsions (see red arrow) appears at the drop top due to evaporation-triggered nucleation. The light scattering by the nucleated microdroplets leads to the white color of the emulsions. In C and D, the white emulsions are more and more visible and start to spread around the entire drop. Finally, the entire drop is opaque in E. F) Explanation diagram of experimental snapshots A-E. The distribution of the evaporation flux and the different volatilities of water and ethanol codetermine that "ouzo effect " preferentially happens at the drop apex. Marangoni flow spreads the nucleated oil microdroplet through the entire drop.

was recorded by a CCD camera [XIMEA; MD061MU-SY, 3 frames per second (fps) at $1,372 \times 1,100$ pixel resolution] equipped with a high-magnification zoom lens system (THORLABS; MVL12X3Z) for side-view recordings and a CMOS [NIKON; D750, 24 frames per seconds (fps) at 1,920 $\times 1,080$ pixels resolution] attached to an identical lens system for top-view recordings. We used a self-built collimated LED source system to illuminate the side-view recording. A powerful Hella LED light source was used for the top-view illumination to show the top of the drop (Figs. 3.1A-E). The relative humidity and temperature in the laboratory were monitored at a sampling rate of one per second with a universal handheld test instrument (OMEGA; HH-USD-RP1, accuracy relative humidity is $\pm 2 \%$ over 10 to $90 \% @ 25^{\circ} \mathrm{C}$ and a temperature accuracy of $\pm 0.3 \mathrm{~K} @ 25^{\circ} \mathrm{C}$ ). The location of the probe was around $10 \mathrm{~cm}$ away from the droplet. A similar sketch of the setup is described in detail in reference [101]. Stereo-imaging was performed by a confocal microscope (Nikon Confocal Microscopes A1 system) with a $20 \times$ air objective (CFI Plan Apochromat VC $20 \times / 0.75$ DIC, NA $=0.75, \mathrm{WD}=1.0 \mathrm{~mm}$ ). Perylene (SIGMA-ALDRICH; sublimed grade, $\geq 99.5 \%$ ) was used to label trans-anethole. The presented $3 \mathrm{D}$ images have a resolution of $1.2 \mu \mathrm{m}$ in the horizontal plane and a vertical resolution of $700 \mathrm{~nm}$. We applied the pendant drop method to measure the interfacial surface tension between different two 
phases (air, water or trans-anethole) with a video-based optical contact angle measuring system (DataPhysics OCA15 Pro).

\subsubsection{Image Analysis and Data Calculation}

Images were analyzed by a custom made MATLAB program. The initial contact line position of the drop was used as input for each data group and automatically adjusted during the subsequent frames. The program fits an ellipse to the contour of the drop in side view. The contact angle $\theta$ was calculated based on position of the intersection of the base line and the fitted ellipse for each frame. The volume $V$ of the drop was obtained by integrating the areas of horizontal disk layers with the assumption that each horizontal layer fulfills rotational symmetry.

\subsection{Experimental results}

\subsubsection{Evaporation-triggered Nucleation at the Top of an Evaporating Ouzo Droplet}

Figures 3.1A-E display an evaporating ouzo drop with low wettability on a superamphiphobic substrate under ambient conditions. Initially, cf. Figure 3.1A, the ouzo drop is transparent and concentrates illuminating light on the substrate. Around $t=t_{0}+167 \mathrm{~s}$, the "ouzo effect" sets in at the top of the drop and a region with a cloudy white emulsion appears (Fig. 3.1B). The nucleated microdroplets scatter the light, giving them "milky" appearance. In Figures $3.1 \mathrm{CD}$, the emulsion is more and more evident and spreading around the drop. Finally, the entire drop is opaque, and the bright spot on the substrate disappears completely (Fig. 3.1E). Experimental movies (SV1 and SV2) and numerical movie (SV3) are available as supplementary material.

The physical origin of the phenomenon has two aspects. One is the maximum local evaporation rate at the top of the drop. On a superamphiphobic substrate, the ouzo drop maintains its high contact angle $\left(\sim 150^{\circ}\right)$ during the evaporation process. Under the assumption of a small temperature gradient along the liquid-air interface, this geometric configuration gives the highest local evaporation flux at the top of the drop [32, 35, 102] (Fig. 3.1F). The thermal gradient along the liquid-air interface is reduced by the strong solutal and thermal Marangoni convection, as discussed in detail in section 3.5.2. The average temperature at the interface is lower than ambient temperature due to evaporative cooling (section 3.5.3).

The second aspect is that the component ethanol in the ouzo drop has a higher volatility than water, while trans-anethole is non-volatile. As the drop 
evaporates, the highest water concentration initially appears at the drop top with the highest local evaporation flux. Hence, the evaporation-triggered ouzo effect [101] starts at the apex of the ouzo drop. The nonuniform concentration field induces surface tension gradient along the interface towards the top, which leads to intense solutal Marangoni convection. The flow drives nucleated oil microdroplets around the drop. After some time, the oil microdroplets nucleate in the whole drop. In the end, numerous oil microdroplets fill up the ouzo drop and scattering the light, creating the milky appearance of the drop.

\subsubsection{Evaporation Phases}

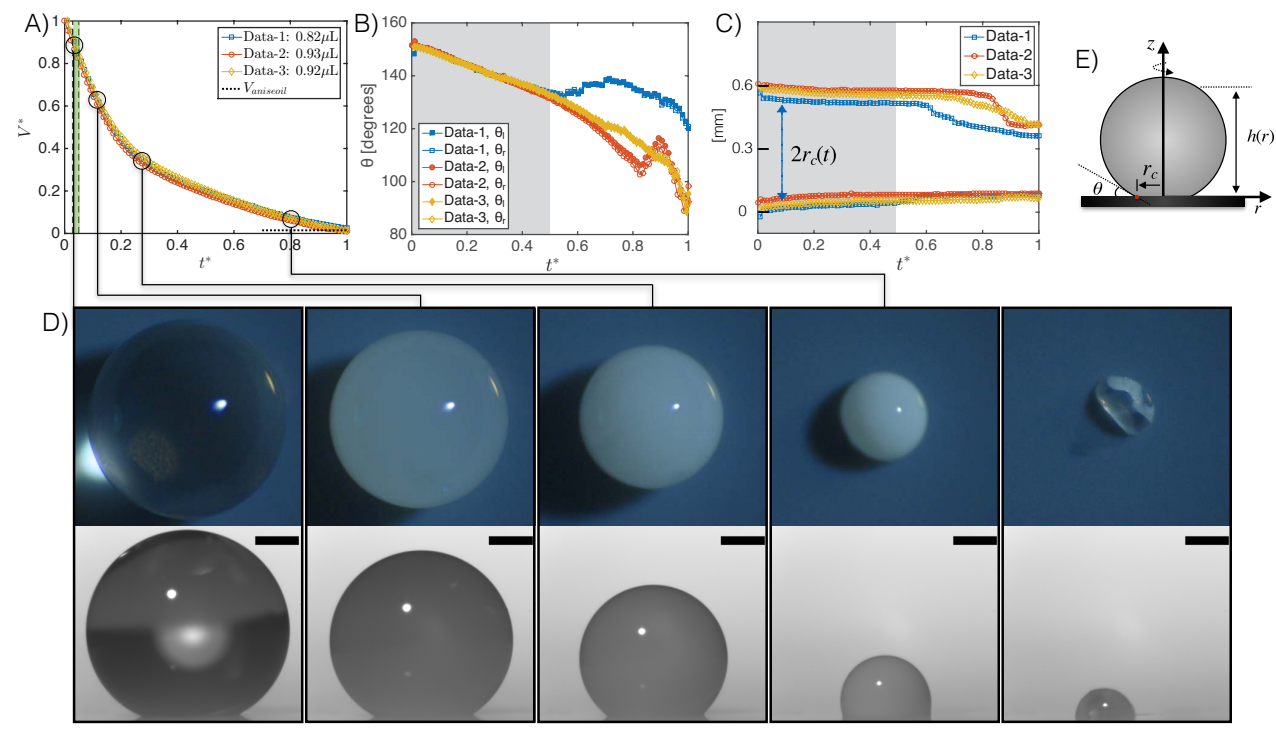

Figure 3.2: A) Temporal evolution of the ouzo drop volume during the evaporation on the surperamphiphobic surface for different initial drop sizes. The drop volumes are nondimensionalized with respect to the initial volume, while the time is nondimensionalized with respect to the total time of water and ethanol evaporation. Two distinct volume evolution slopes are evident. The time interval marked green indicates approximately the transition of the ouzo drops from transparent to opaque. B) Temporal evolution of the contact angle. For each data group, left and right contact angles are in good agreement, i.e. no bias, at an early stage (grey region), while different evolutions appear afterwards. C) Temporal evolution of the size of the contact area, characterized by $2 r_{c}(t)$. For the ouzo drops in our case the CR-model is roughly applicable at the early stage (grey region). D) Experimental snapshots (up: top view; bottom: side view) ${ }^{\S}$ at different moments related to panel A. The scale bar is $\mid 0.25 \mathrm{~mm}$. The last group snapshot shows the residual drop after evaporation recording. Only oil is left. E) The sketch of an ouzo drop with annotations.

${ }^{\dagger} \S$ With respect to the top view snapshots in Fig.2D, the side-view recording was recorded 

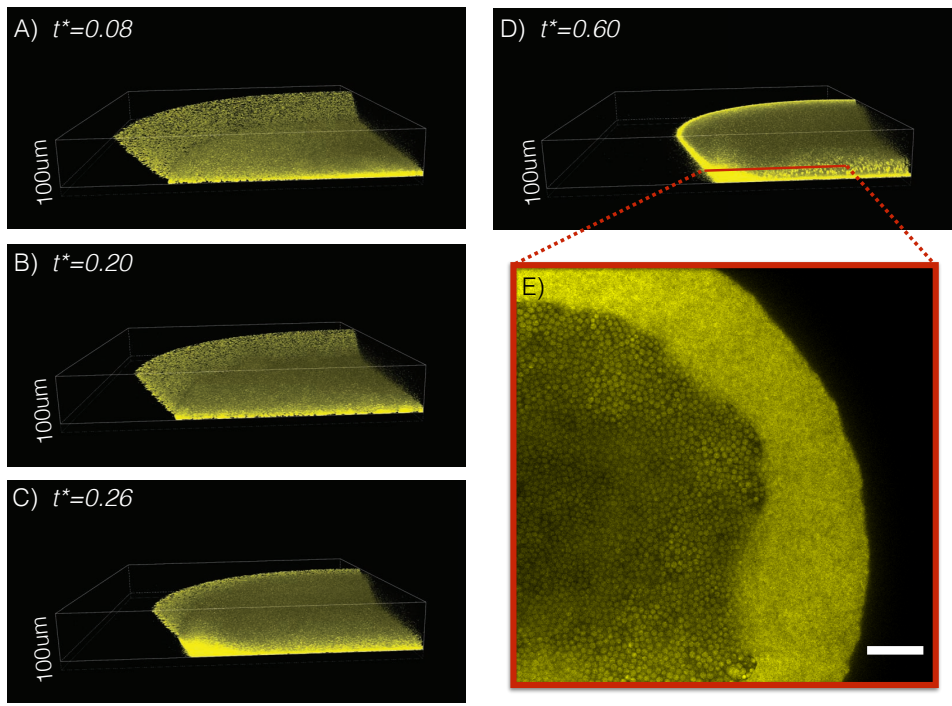

Figure 3.3: Wrapping of nucleated oil in the evaporating ouzo drop. A-D) Three-dimensional confocal images of the contact region of the ouzo drop at different moments with $20 \times$ air objective. After oil nucleation, nucleated oil microdroplets (yellow) fill up the ouzo drop. The microdroplets on the bottom and near the surface are much more visible than the ones inside because of the direct laser excitation without light refraction. In panels A and B, as the ouzo drop evaporates, the contact angle decreases with apparent movement of the contact line. In $\mathrm{C}$, the accumulated oil microdroplets fuse into a temporary oil ring at the rim. After a while $\left(t^{*}=0.6\right.$ and ethanol has almost entirely evaporated), an oil shell wraps up the ouzo drop, as shown in D. E) A high resolution two-dimensional confocal snapshot inside the drop with a $60 \times$ oil immersion objective. Numerous nucleated oil microdroplets in water phase surroundings are capsuled by the oil shell. The scale bar is $20 \mu \mathrm{m}$. The microdroplets have a diameter size around $\sim 2 \mu \mathrm{m}$.

We monitored evaporating ouzo drops with initial volumes $V_{\text {init }}$ of 0.82 , 0.93 or $0.92 \mu \mathrm{L}$ to study its evaporation characteristics, including the transient drop volume $V$, the contact angle $\theta$, and the radius of the contact line $r_{c}$ (as annotated in Figure $3.2 \mathrm{E}$ ). Here, we nondimensionalized the temporal evolution of the drop volume as follows: we define a nondimensional volume $V^{*}$ by dividing the drop transient volume $V$ by the original amount $V_{\text {init }}$. A nondimensional time $t^{*}$ for the first three phases is determined by dividing time $t$ by the total time of the water/ethanol evaporation time $(\sim 660 \mathrm{~s}, \sim$ $727 \mathrm{~s}$ and $\sim 736 \mathrm{~s}$ for Data-1, Data-2, and Data-3, respectively). After the nondimensionalization, the three temporal evolution curves overlap, as shown in Figure 3.2 A.

Like on flat hydrophobic surfaces [101] two distinct time regimes could be

from the right side by the camera placed upside down. This was due to the equipment installation and the setup arrangement in our experiments. 
distinguished for drops evaporating from superamphiphobic surfaces (Fig. 3.2 A). The similar feature also exists for an evaporating water-ethanol drop in gas [56, 57, 59] and a dissolving sessile drop in an ouzo system [105]. The difference of evaporation/diffusion rates of the components in the drop determines this feature. In the first period $\left(t^{*}<0.2\right)$ of the volume evolution curves in Figure $3.2 \mathrm{~A}$, the ouzo drop undergoes a transition from phase I to phase II, i.e. from transparent (first snapshot column in Fig. 3.2D) to opaque (second snapshot column in Fig. 3.2D). For the ouzo drops in Data-1, Data-2 and Data-3, the onset of the transition is at $\sim 21 \mathrm{~s}, \sim 21 \mathrm{~s}$ and $\sim 23 \mathrm{~s}$ after being deposited on the surface, respectively. The transitions take $\sim 12 \mathrm{~s}, \sim 12 \mathrm{~s}$, and $\sim 17 \mathrm{~s}$ accordingly to finish the transition. The transition interval (green region in Fig. 3.2 A) is short compared to the whole evaporation process. The steep decrease of the initial volume results from the high volatililty of ethanol. During this period, the contact angle smoothly drops from $\sim 150^{\circ}$ to $\sim 140^{\circ}$ (Fig. 3.2 B), whereas the contact radius $r_{c}$ has a subtle shift (Fig. $3.2 \mathrm{C}$ ), i.e. the drop is roughly in the Constant Radius (CR)-mode [37. We note that not only the left and right contact angles of a single drop coincide, but also the contact angles of different drops. Furthermore, three-phase intersection points in the side-view contour, which were reported in our previous study on flat evaporating ouzo drops [101, are now absent.

When most of the ethanol has evaporated, the evaporation of water dominates and determines the less steep slope of phase III. The ouzo drop is milky during the whole phase III (the third and fourth snapshot columns in Fig. 3.2 D). There is no phase inversion from oil droplets in water to water droplets in oil as was reported for flat evaporating ouzo drops [101]. The nucleated oil emulsion microdroplets fill up the ouzo drop and remain stable. After some time, the contact angle increases and the contact area simultaneously shrinks to a smaller base radius (Figs. $3.2 \mathrm{BC}$ ), i.e. the CR-mode ceases. As the water is evaporating, its concentration in the drop continues to decrease until there is not enough water to maintain the stability of oil emulsion microdroplets in the bulk. The microdroplets then start to coalesce (last snapshot column in Fig. 3.2D), leaving behind a small trans-anethole drop. The capillary force at the contact line on the surface can damage the soot coating layer. The residual trans-anethole drop rolls up the damaged layer, resulting in a non-spherical-cap shape in the end.

\subsubsection{Wrapping of Nucleated Oil}

As discussed before, the drop initially is in CR-mode [37] in the early stages of the evolution (for example, $t^{*}<0.5$ for Data- 1 ). To further investigate this behavior, we used a confocal microscope to image the contact region of an 
evaporating ouzo drop. Figures 3.3 AB show the nucleated oil microdroplets (yellow) in the bulk or on the surface. The presence of numerous oil microdroplet leads to a high light absorption, which shields the oil microdroplets inside the ouzo drop. Thanks to the visibility of oil microdroplets along the drop surface, the behavior of the drop surface in the contact region is detectable. The confocal images confirm that at early time $\left(t^{*} \leq 0.2\right)$ the contact angle of the drop decreases and the contact line is fixed. At some point later, inward movement of the contact line facilitates the coalescence of the nucleated oil microdroplet on the surface, forming a temporary oil ring as displayed in Figure $3.3 \mathrm{C}$.

The oil ring does not persist for a long time. It starts to climb along the liquid-air interface and wraps up the ouzo drop at some point when the drop has a small ethanol concentration and a high surface energy. Smith et al. [106] and Schellenberger et al. [107] have reported the same phenomenon before. The spreading coefficient gives a criterion for the occurrence of the wrapping process [106. It is defined as $S_{\mathrm{ta}, \mathrm{l}} \equiv \gamma_{1, \mathrm{a}}-\gamma_{\mathrm{ta}, \mathrm{a}}-\gamma_{1, \mathrm{ta}}$, where $\gamma$ is the interfacial tension between different phases, 1 is short for the ouzo drop liquid, a is for air, and ta is for trans-anethole [108]. If $S_{\mathrm{ta}, \mathrm{w}}$ is positive, the trans-anethole-air interface and trans-anethole-liquid together have a lower energy than the liquid-air interface and thus trans-anethole can wrap up the drop [109]. Table 3.1 lists the interfacial tension between water and air $\left(\gamma_{\mathrm{w}, \mathrm{a}}\right)$, trans-anethole and air $\left(\gamma_{\mathrm{ta}, \mathrm{a}}\right)$, and water and trans-anethole $\left(\gamma_{\mathrm{w}, \mathrm{ta}}\right)$. If the drop liquid is water, the spreading coefficient $S_{\mathrm{ta}, \mathrm{w}}$ is positive. Consequently, the spreading parameter predicts total wetting and the trans-anethole wraps up the ouzo drop completely. As shown in Figure 3.3D, a bright oil shell appears. An experimental movie (SV4) created by confocal microscope is available as supplementary material. To have an observation inside the ouzo drop at high resolution, we performed a $2 \mathrm{D}$ scan with an $60 \times$ oil immersion objective in the confocal microscope system. Figure $3.3 \mathrm{E}$ shows a snapshot, where we find numerous nucleated oil microdroplets with $\sim 2 \mu \mathrm{m}$ diameter size by the continuous oil shell. Here, only fluorescent dye was added for trans-anethole.

Table 3.1: Interfacial surface tension between different two phases: a is short for air, w for water, and ta for trans-anethole.

\begin{tabular}{cccc}
\hline$\gamma_{\mathrm{w}, \mathrm{a}}[\mathrm{mN} / \mathrm{m}]$ & $\gamma_{\mathrm{ta}, \mathrm{a}}[\mathrm{mN} / \mathrm{m}]$ & $\gamma_{\mathrm{w}, \mathrm{ta}}[\mathrm{mN} / \mathrm{m}]$ & $S_{\mathrm{ta}, \mathrm{w}}[\mathrm{mN} / \mathrm{m}]$ \\
\hline 72 & 35.5 & 24.2 & $>0$ \\
\hline
\end{tabular}




\subsection{FEM numerical modeling for evaporation}

Additional insight in the entire process can be obtained by numerical modeling. Since the initial contact angle is higher than $90^{\circ}$, the lubrication theory model of references [101, 110] cannot be used. To overcome this limitation and to provide an accurate prediction of the flow velocity even at high contact angles, a new finite element method (FEM) model has been developed. Here, we only give an outline of this model, and details can be found in reference [111. In order to allow for acceptable calculation times, the model assumes axisymmetry. Furthermore, it is assumed that the drop is always in a spherical-cap shape and consists of a miscible liquid mixture. When the ouzo effect occurs, the latter assumption is still valid as long as the oil microdroplets are small compared to the entire drop. In the simulations, the ouzo effect is defined to happen when the local composition is in the experimentally determined ouzo effect regime of the ternary phase diagram of reference [101].
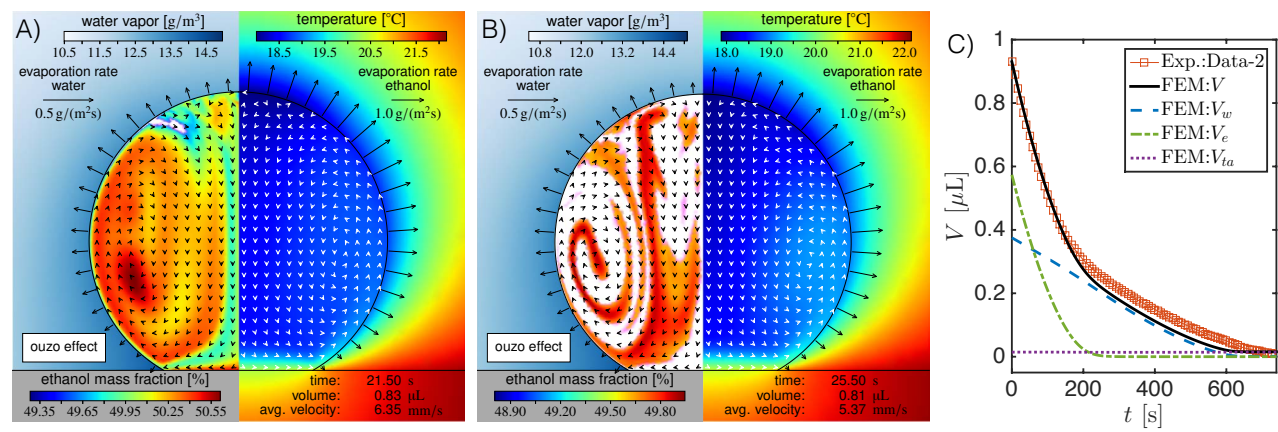

Figure 3.4: Snapshots of the simulation at different times, $t=21.5 \mathrm{~s}(\mathrm{~A})$ and $t=25.5 \mathrm{~s}(\mathrm{~B})$, and good agreement between volume evolutions from experiments and numerical simulations (C). Snapshots A-B) The left side shows water vapor concentration $c_{\mathrm{w}}$ in the gas phase and ethanol mass fraction $y_{\mathrm{e}}$ in the droplet and the right side presents temperature field. The arrows in the drop indicate the flow direction and the evaporation rates $j_{\mathrm{w}}$ and $j_{\mathrm{e}}$ of water and ethanol are indicated by the interface arrows on the left and right side, respectively. At about $t=21.5 \mathrm{~s}$, the ouzo effect occurs at the apex of the drop (indicated by white regions on the left side). At about $t=27.2 \mathrm{~s}$, the phase separation is occurring in the entire drop. C) shows good agreement of volume evolutions of experimental data (square symbols) and simulations (black solid line). During the first 200 seconds, the volume loss is predominantly constituted by the evaporation of ethanol (green dash-dotted line), while in the following, the evaporation of water (blue dashed line) determines the volume loss rate.

The model solves the coupled processes of multi-component evaporation, Stokes flow in the droplet driven by solutal and thermal Marangoni flow and convection-diffusion equations for the spatio-temporal liquid composition and the temperature. The composition-dependence of the liquid properties, i.e. mass density, viscosity, surface tension, diffusivity and thermodynamic activ- 
ities, are taken into account. However, since these relations are not available for the ternary mixture, experimental data of binary water-ethanol mixtures [67-69] have been fitted to model the composition-dependence. The thermodynamic activities of water and ethanol were calculated by AIOMFAC [70, 71]. Plots of the fitted relations and description of all used parameters can be found in supplementary material.

The evaporation rate for component $\nu(\nu=\mathrm{w}, \mathrm{e}$ for water and ethanol, respectively) are calculated by solving the vapor diffusion equation $\partial_{t} c_{\nu}=$ $D_{\nu, \text { air }}^{\text {vap }} \nabla^{2} c_{\nu}$ in the gas phase, where $D_{\nu, \text { air }}^{\text {vap }}$ is the vapor diffusivity of $\nu$ in air and $c_{\nu}$ is the vapor concentration, i.e. the partial density. With the ideal gas law, one can express the ambient water vapor concentration by

$$
c_{\mathrm{w}, \infty}=H \frac{M_{\mathrm{w}} p_{\mathrm{w}, \mathrm{sat}}\left(T_{\infty}\right)}{R T_{\infty}},
$$

where $H$ is the relative humidity, $R$ is the ideal gas constant, $T_{\infty}$ is the room temperature and $p_{\mathrm{w} \text {,sat }}$ is the saturation pressure, which temperaturedependence given by the Antoine equation. There is no ambient ethanol vapor present, i.e. $c_{\mathrm{e}, \infty}=0$. At the liquid-gas interface, the vapor-liquid equilibrium according to Raoult's law has to hold,

$$
c_{\nu, \mathrm{VLE}}=\gamma_{\nu} x_{\nu} \frac{M_{\nu} p_{\nu, \mathrm{sat}}(T)}{R T} .
$$

By virtue of equation $(3.2)$, the evaporation rates are coupled with the local drop composition at the interface via the liquid mole fraction $x_{\nu}$ and the activity coefficient $\gamma_{\nu}$ and furthermore with the local temperature $T$. From the diffusive vapor fluxes $J_{\nu}^{\text {gas }}=-D_{\nu \text {,air }}^{\text {vap }} \partial_{n} c_{\nu}$ at the interface, the mass transfer rates $j_{\mathrm{w}}$ and $j_{\mathrm{e}}$ can be determined from the coupled mass transfer jump conditions

$$
j_{\nu}-J_{\nu}^{\text {gas }}-\frac{c_{\nu}}{\rho_{\text {air }}}\left(j_{\mathrm{e}}+j_{\mathrm{w}}\right)=0 .
$$

Here, the mass density $\rho_{\text {air }}$ of the gas phase is assumed to be constant and given by the value of humid air.

The drop is assumed to be in a spherical-cap shape, where the base contact radius $r_{\mathrm{c}}$ was adjusted according to the experimental data (Data-2), i.e. by fitting $r_{\mathrm{c}}(V)$. This allows to calculate the normal interface velocity from the evaporative volume loss via the kinematic boundary condition. Together with the tangential Marangoni shear stress boundary condition, the Stokes flow can be solved in the drop. The obtained velocity $\vec{u}$ is entering the convectiondiffusion equations for the composition, expressed in terms of mass fractions $y_{\nu}$, i.e.

$$
\rho\left(\partial_{t} y_{\nu}+\vec{u} \cdot \nabla y_{\nu}\right)=\nabla \cdot\left(\rho D \nabla y_{\nu}\right)-J_{\nu} \delta_{\Gamma},
$$


with the mixture diffusivity $D$ and the mass transfer source/sink term imposed at the liquid-gas interface $\Gamma$. The source/sink term is given by

$$
J_{\nu}=j_{\nu}-y_{\nu}\left(j_{\mathrm{e}}+j_{\mathrm{w}}\right) .
$$

The mass fraction $y_{\mathrm{ta}}$ of trans-anethole oil is obtained by $y_{\mathrm{ta}}=1-y_{\mathrm{w}}-y_{\mathrm{e}}$.

Finally, the following temperature equation has to be solved:

$$
\rho c_{p}\left(\partial_{t} T+\vec{u} \cdot \nabla T\right)=\nabla \cdot(\lambda \nabla T)-\left(\Lambda_{\mathrm{w}} j_{\mathrm{w}}+\Lambda_{\mathrm{e}} j_{\mathrm{e}}\right) \delta_{\Gamma} .
$$

Here, $\Lambda_{\nu}$ is the latent heat of evaporation, $\rho$ is the mass density, $c_{p}$ the specific heat capacity and $\lambda$ is the thermal conductivity. These quantities are different in the different domains, i.e. gas phase, drop and substrate. In order to accurately reproduce the thermal conduction of the experimental setup, a fused quartz substrate with a finite thickness of $1.17 \mathrm{~mm}$ and air below is considered.

The simulation results confirm the interpretation discussed in section 3.3 .1 on the phenomenon that the evaporation-triggered nucleation starts to occur at the top of an evaporating ouzo drop. The snapshots of the simulation (Figs. 3.4 AB) shows that the ouzo effect indeed sets in close to the apex at about $t=20 \mathrm{~s}$ (white regions in Fig. 3.4 A). At $t=26.3 \mathrm{~s}$, phase separation is occurring in the entire drop. Figure $3.4 \mathrm{C}$ presents the temporal evolution of the drop volume and the partial volume of each component. The two distinct slopes in the drop volume curve in the early stage and at later stages correspond to the curve slopes of ethanol and water, respectively. Furthermore, the FEM simulation provides more information about the evaporating ouzo drop. Due to the enhanced evaporation at the top of the drop, both the temperature and the ethanol concentration have their minimum close to the apex. Since both aspects increase the local surface tension, a strong Marangoni flow is induced from the contact line along the interface towards the top. As a result of the solutal Marangoni instability, however, the flow in the drop is, in general, not regular, but exhibits chaotic behavior until almost all ethanol has evaporated. Thus, one also has to expect axial symmetry breaking, which is discussed in more detail in a forthcoming three-dimensional investigation of the evaporating ouzo drop [112].

\subsection{Generalized diffusion model for ouzo drops}

As stated above, an analytical diffusion model for quasi-steady natural evaporation of one-component drops was proposed by Popov [32]. To generalize Popov's diffusion model for the evaporation process of ouzo drops with more than one component, we take account of Raoult's law, which is necessary for 
building up the vapor-liquid equilibrium at the drop interface [101, 110]. We assume the liquid solution in the drop to be well-mixed as a result of the existence of the strong Marangoni flow [101, 112. The mixed-convection flow gives rise to both a uniform concentration field and a uniform thermal field inside the drop, which is discussed in detail in section 3.5.2. In the generalized model, the temperature dependence of the vapor concentration of each component is also considered, because evaporative cooling effects at the drop interface is obvious for an evaporating drop at a high contact angle [34. Detailed discussion is presented in section 3.5 .3 .
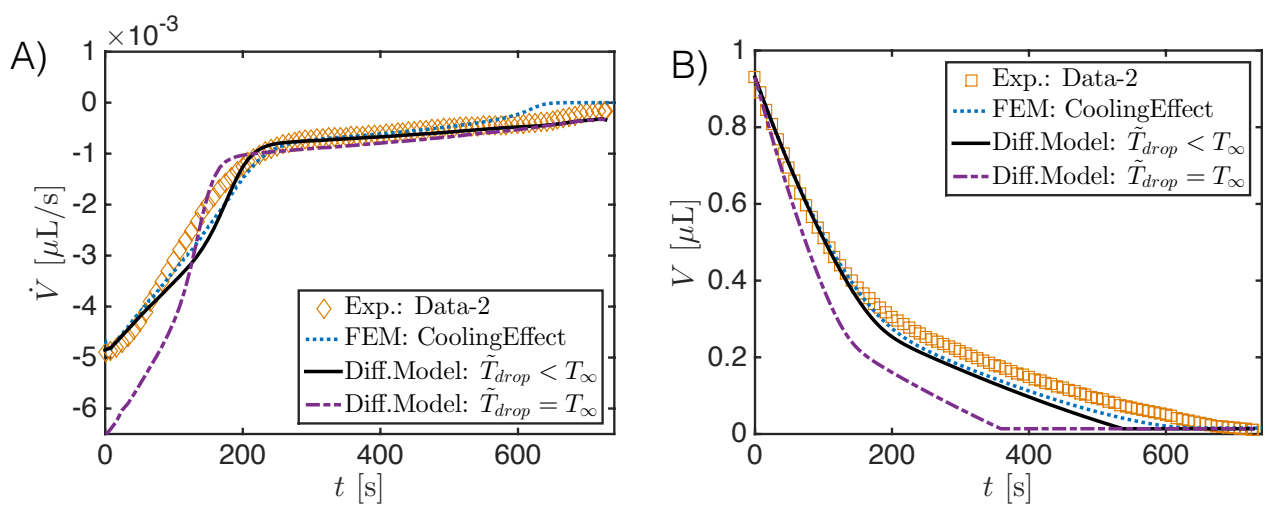

Figure 3.5: Evolutions of volume loss rate (panel A) and drop size (panel B) calculated from the approximate diffusion model $\left(\tilde{T}_{\text {drop }}<T_{\infty}\right)$, displayed in black solid lines, and the model without cooling-effect consideration $\left(\tilde{T}_{\text {drop }}=T_{\infty}\right)$, presented in purple dash-dotted lines. The blue dotted lines are the results from the FEM simulation (cooling effect included). The model results are in a good accordance with experimental data and FEM simulation results. When the approximate diffusion model excludes the evaporative cooling effect, the satisfaction to the experimental data and simulation results lose, and the calculated volume loss rates are higher, especially in the early stage where ethanol evaporation dominates.

\subsubsection{Generalized Diffusion Model}

In Popov's model, the evaporation flux $J(r)$ on the surface of a pure fluid drop is given by 32 .

$$
\begin{aligned}
& J(r)=\frac{D_{\text {air }}^{\mathrm{vap}}\left(c_{\mathrm{sat}}-c_{\infty}\right)}{r_{c}}\left[\frac{1}{2} \sin \theta+\sqrt{2}(\cosh \alpha+\cos \theta)^{3 / 2}\right. \\
& \left.\times \int_{0}^{\infty} \frac{\cosh \theta \tau}{\cosh \pi \tau} \tanh [(\pi-\theta) \tau] P_{-1 / 2+i \tau}(\cosh \alpha) \tau \mathrm{d} \tau\right],
\end{aligned}
$$


where $r=\frac{r_{c} \sinh \alpha}{\cosh \alpha+\cos \theta}$ is the radial coordinate at the surface of the drop, $r_{c}$ is the contact radius of the drop, $D_{\text {air }}^{\text {vap }}$ is the coefficient of vapor diffusion, $c_{\mathrm{s}}$ is the saturated vapor concentration on the drop surface, $c_{\infty}$ is the concentration of vapor at infinity, and $\theta$ is contact angle (cf. sketch in Fig. $3.2 \mathrm{E}$ ).

To generalize the model for ouzo drops, the evaporation flux along the vapor-liquid interface of each component $\nu$ can be expressed as

$$
\begin{gathered}
J_{\nu}(r)=\frac{D_{\nu, \mathrm{air}}^{\mathrm{vap}}\left(c_{\nu, \mathrm{VLE}}-c_{\nu, \infty}\right)}{r_{c}}\left[\frac{1}{2} \sin \theta+\sqrt{2}(\cosh \alpha+\cos \theta)^{3 / 2}\right. \\
\left.\quad \times \int_{0}^{\infty} \frac{\cosh \theta \tau}{\cosh \pi \tau} \tanh [(\pi-\theta) \tau] P_{-1 / 2+i \tau}(\cosh \alpha) \tau \mathrm{d} \tau\right]
\end{gathered}
$$

where the concentration of each component at the vapor-liquid interface is determined by Raoult's law, i.e. equation (2), $c_{\nu, \mathrm{VLE}}=\gamma_{\nu} x_{\nu} c_{\nu, \mathrm{sat}}$.

The evaporation rate of the mass of each component $\dot{m}_{\nu}$ is expressed as an integral of the evaporation flux over the drop surface, i.e.,

$$
\dot{m}_{\nu}=-\int_{0}^{r_{c}} J_{\nu}(r) \sqrt{1+\left(\partial_{r} h(r)\right)^{2}} 2 \pi r \mathrm{~d} r .
$$

By virtue of the assumption of uniform concentration and homogeneous temperature along the interface, both vapor concentration $c_{\nu, \mathrm{VLE}}$ at the drop surface and vapor diffusivity $D_{\nu \text {,air }}^{\text {vap }}$ are independent of position coordinate $r$. The concentration $c_{\nu, \mathrm{VLE}}$ depends on the averaged surface temperature $\tilde{T}_{\text {drop }}$ of the drop. Both the diffusivity $D_{\nu \text {,air }}^{\text {vap }}$ and the ambient vapor concentration $c_{\nu, \infty}$ are determined by room temperature $T_{\infty}$. Therefore, the evaporation rate of drop mass $\dot{m}$ can be expressed as follows:

$$
\begin{gathered}
\dot{m}_{\nu}=-\pi r_{c} D_{\nu, \text { air }}^{\text {vap }}\left(c_{\nu, \mathrm{VLE}}\left(\tilde{T}_{\text {drop }}\right)-c_{\nu, \infty}\left(T_{\infty}\right)\right) f(\theta), \\
c_{\nu, \mathrm{VLE}}\left(\tilde{T}_{\mathrm{drop}}\right)=\gamma_{\nu}\left(\tilde{T}_{\mathrm{drop}}\right) x_{\nu} \frac{M_{\nu} P_{\nu, \mathrm{sat}}\left(\tilde{T}_{\mathrm{drop}}\right)}{R \tilde{T}_{\text {drop }}}, \\
f(\theta)=\frac{\sin \theta}{1+\cos \theta}+4 \int_{0}^{\infty} \frac{1+\cosh 2 \theta \tau}{\sinh 2 \pi \tau} \tanh [(\pi-\theta) \tau] \mathrm{d} \tau .
\end{gathered}
$$

The evaporation rate of the drop mass $\dot{m}$ is given by $\dot{m}=\sum \dot{m}_{\nu}=\sum \rho_{\nu} \dot{V}_{\nu}$, where $\dot{V}_{\nu}$ is the evaporation rate of each component volume. When the small volume-change caused by the mixture of miscible liquids is ignored, the evaporation rate of the drop volume $\dot{V}$ can be expressed as,

$$
\dot{V}=\sum \frac{1}{\rho_{\nu}} \dot{m}_{\nu}
$$


In the application of the generalized diffusion model for the evaporation process of an ouzo drop, we assume that the trans-anethole $(\nu=$ ta) is nonvolatile and has no influence on the evaporation of water $(\nu=\mathrm{w})$ and ethanol $(\nu=\mathrm{e})$. For water $(\nu=\mathrm{w})$, the ambient vapor concentration $c_{\mathrm{w}, \infty}$ is given by $H c_{\mathrm{w}, \mathrm{sat}}\left(T_{\infty}\right)$, i.e. equation (3.1). For ethanol $(\nu=\mathrm{e})$, we assume that the ambient vapor concentration $c_{\mathrm{e}, \infty}$ is zero, thus its evaporation rate is independent of the $H$ factor. The saturation pressure $P_{\mathrm{w}, \text { sat }}$ and $P_{\mathrm{e}, \text { sat }}$ in equations (3.1) and (3.2) at different temperatures are calculated from the Antoine equation. The activity coefficients $\gamma_{\nu}$ are calculated from the Dortmund Data Bank with the lastest available parameters [113. Vapor diffusivity $D_{\mathrm{w} \text {, air }}^{\mathrm{vap}}$ at different temperatures is obtained from reference [64] by cubic spline interpolation. The vapor diffusivity $D_{\mathrm{e}, \text { air }}^{\mathrm{vap}}$ is calculated based on the equation in reference [114]. We use $\rho_{w}=997.773 \mathrm{~kg} \mathrm{~m}^{-3}, \rho_{e}=786.907 \mathrm{~kg} \mathrm{~m}^{-3}, M_{w}=0.018 \mathrm{~kg} \mathrm{~mol}^{-1}$ and $M_{e}=0.046 \mathrm{~kg} \mathrm{~mol}^{-1}$. The contact radius of the drop $r_{c}$, the contact angle $\theta$, the ambient temperature $T_{\infty}$ and the relative humidity $H$ at each moment were measured in the experiments. The decreased drop temperature $\tilde{T}_{\text {drop }}$ is substituted by the interface-averaged temperature from the FEM simulation in section 3.4. The initial mole fractions of water $x_{\mathrm{w}}$ and ethanol $x_{\mathrm{e}}$ are calculated based on the initial water and ethanol composition of our ouzo liquid. Then $x_{\mathrm{w}}$ and $x_{\mathrm{e}}$ are recalculated in each step base on volume loss rate $\dot{V}_{i}$ and their values in the previous step. The calculation was performed in MATLAB with homemade codes.

Figure 3.5. A shows the evaporation rate of the drop volume predicted by the generalized diffusion model (black solid line) has a good agreement both with the measured experiment data (diamond points) and with FEM simulation results (blue dotted line). However, when the evaporative cooling effect is ignored in the model (purple dash-dotted line), i.e. $\tilde{T}_{\text {drop }}=T_{\infty}$, a clear deviation of the evaporation rates appears, especially at early stage where the ethanol evaporation dominates. This is a result of the high volatility of ethanol which enhances the evaporative cooling effect. At later stages, the deviation disappears caused by two factors. The first factor is the low volatility of water, which reduces the evaporative cooling effect. The second factor is the decreased contact angle of the evaporating drop, as shown in figure $3.2 \mathrm{~B}$. A smaller contact angle gives a smaller temperature reduction at the drop top [34] and also weakens the cooling effect. An integral of the evaporation rate from initial time $t_{0}$ gives the temporal evolution of the drop volume, that is $V(t)=\int_{t_{0}}^{t} \dot{V} \mathrm{~d} t$. As displayed in figure $3.5 \mathrm{~B}$, the diffusion model provides a comparable evolution curve (black solid line) to the one (square points) from experimental data and the one (blue dotted line) from FEM simulation result. There is slight deviation in the later stage, although the slopes of evolutionary 
curves are almost the same. There are two potential reasons for this deviation: (i) The isothermal assumption doesn't satisfy strictly because of the reduced intensity of the Marangoni flow. (ii) The existence of the nucleated oil microdroplet in the drop plays a role. For the case without considering the cooling effect, the temporal evolution curve (purple dash-dotted line) obviously deviates from the other curves. The curve still develops with two different slopes, but the entire evaporation is predicted too fast. It indicates that the evaporative cooling effect is a very important aspect in the evaporation process of the droplet with a high contact angle.

\subsubsection{Strong Marangoni flow}

For an evaporating multi-component drop, the surface tension gradient along the drop surface may be very small, whereas the Marangoni flow can be very strong [101, 110]. For a millimetre water-ethanol drop, the averaged flow velocity inside is of order of $\mathrm{mm} / \mathrm{s}$ [110, 111]. Its Péclet numbers $P e$ for mass transfer and heat transfer are $10^{5}$ and $10^{3}$, respectively, given its mass diffusion coefficient $\left(\sim 10^{-9} \mathrm{~m}^{2} \mathrm{~s}^{-1}\right)$ and thermal diffusivity $\left(\sim 10^{-7} \mathrm{~m}^{2} \mathrm{~s}^{-1}\right)$. Although the inconsistent evaporation flux along the drop surface and the evaporative cooling effect causing the concentration gradient and thermal gradient, respectively, the strong Marangoni flow can dramatically uniformize both the concentration and thermal distribution in the drop. Here we assume that the drop has both a uniform concentration field and a uniform thermal field inside. Then both the vapor concentration $c_{\nu, \mathrm{VLE}}$ at the drop surface and vapor diffusivity $D_{\nu \text {,air }}^{\text {vap }}$ are independent of position coordinate $r$. The entire drop is characterized by an identical temperature value $\tilde{T}_{\text {drop }}$. The concentration $c_{\nu, \mathrm{VLE}}$ only depends on the drop (surface) temperature $\tilde{T}_{\text {drop }}$.

\subsubsection{Evaporative cooling effect at the drop surface}

Evaporative cooling effect is a very important aspect for the evaporation process of sessile drops [34, 95, 115, 117]. During the evaporation, the phase change at the liquid-air interface consumes energy and results in temperature reduction. Meanwhile heat is replenished from the substrate by heat conduction. These two main factors lead to a non-isothermal field in the drop [116]. There is a temperature distribution along the liquid-air interface. The temperature difference within an evaporating drop on hydrophilic surfaces is minimal [115], whereas there is a relative large temperature reduction across the drop on hydrophobic surfaces [34, 95, 117]. All the literature mentioned above are for a single-component drop. 
In our case, the ouzo drop has a large contact angle. It is vital to take account of the temperature reduction caused by evaporative cooling effects. But something different happens here. As discussed in section 3.5.2, the appearance of the strong Marangoni flow uniformizes the temperature field. It is possible to have a thermal boundary layer along the substrate surface. The estimation of its thickness is $\sim 100 \mu \mathrm{m}$, given by $P e=1$. And as a result there is no distinct temperature difference in most parts of the drop, as displayed in Figures 3.4AB. Therefore it is reasonable to assume an isothermal drop with a reduced temperature value.

\subsection{Conclusions}

The evaporation of an ouzo drop on a superamphiphobic surface is characterized by three features: (i) Nucleation of oil microdroplets triggered at the top of the drop. (ii) Two distinct regimes in the evaporation rates can be distinguished, with the formed oil wrapping around the ouzo drop. (iii) In the final stage of evaporation a continuous oil phase cloaks the drop. Quantitative results for the temporal evolution of the drop volume, contact angle and the size of contact area are presented. A numerical simulation with a new FEM method [111] verifies the evaporation-triggered nucleation at the drop top and provides additional insight into the entire physical process. Although the inconsistent evaporation flux along the drop surface and the evaporative cooling effect cause the concentration gradient and thermal gradient, respectively, the Marangoni flow dramatically equalizes both the concentration and thermal gradients in the drop. Taking advantage of the uniformity inside the drop, we propose a generalized diffusion model for the evaporation process of an ouzo drop based on Popov's theory. We generated a model by integrating Raoult's law and the evaporative cooling effect and then simplified the model with the uniformity assumption. The proposed model provides a simplified way to analyze the evaporation process of multi-component drops. With the experimental data and FEM simulation results the predicted instantaneous volume of the ouzo drop shows good agreement. It is appropriate to build up a proper model for the ouzo drop temperature distribution to complete the model. This work highlights the influence of substrate on the evaporation process of ouzo drops. A better understanding of the dynamics of an evaporating ouzo drop may provide valuable information for the investigation of the evaporation process of multi-component mixture drops. 


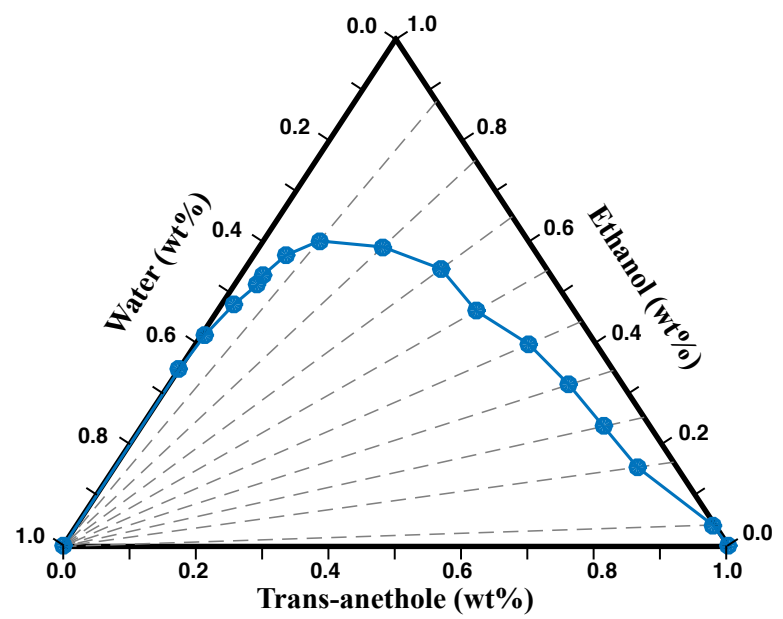

Figure 3.6: Phase diagram for the trans-anethole-ethanol-water system. The blue dots present the measured miscibility limit.

\subsection{Supplementary material}

\subsubsection{Phase diagram of the trans-anethole-ethanol-water system}

Figure 3.6 is the ternary diagram of the trans-anethole-ethanol-water system. The blue solid line is the measured phase-separation curve. The gray dashed lines indicate the composition paths of the titration experiments. The titration was conducted at a temperature of around $22^{\circ} \mathrm{C}$.
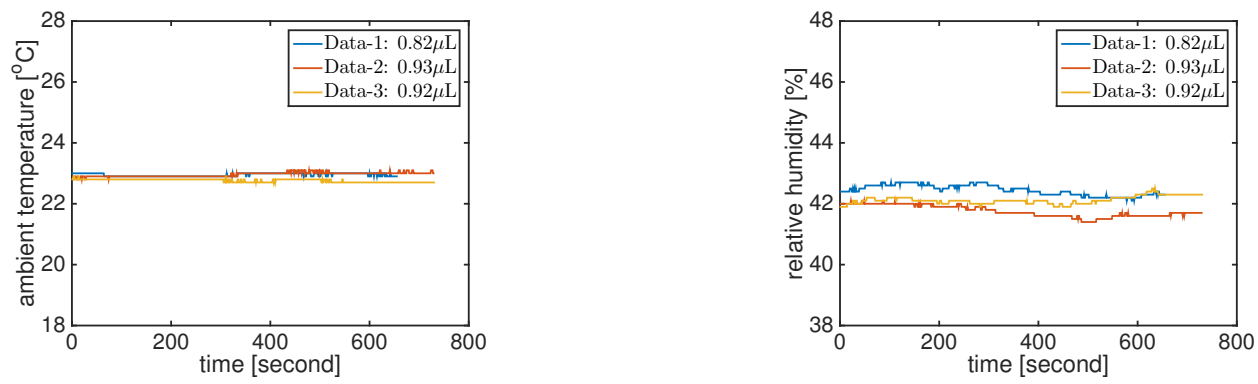

Figure 3.7: Measured ambient temperature $T_{\infty}$ and relative humidity $H$ during the evaporation experiments.

\subsubsection{Temperature and relative humidity}

Figure 3.7 presents the temperature $T_{\infty}$ and relative humidity $H$ in the laboratory during the experiments. The sampling rate is one per second with a 
relative humidity accuracy of $\pm 2 \%$ over 10 to $90 \% @ 25^{\circ} \mathrm{C}$ and a temperature accuracy of $\pm 0.3 \mathrm{~K} @ 25^{\circ} \mathrm{C}$ ). 


\section{4 \\ Dissolving ouzo drops $\overbrace{}^{\circledast}$}

Multicomponent liquid drops in a host liquid are very relevant in various technological applications. Their dissolution or growth dynamics is complex. Difference in solubility between the drop components combined with the solutal Marangoni effect and natural convections contribute to this complexity, which can be even further increased in combination with the ouzo effect, i.e. the spontaneous nucleation of microdroplets due to composition-dependent miscibilities in a ternary system. The quantitive understanding of this combined process is important for applications in industry, particularly for modern liquid-liquid microextraction processes. In this work, as a model system, we experimentally and theoretically explore water/ethanol drops dissolving in anethole oil. During the dissolution, we observed two types of microdroplets nucleation, namely water microdroplet nucleation in the surrounding oil at drop midheight and oil microdroplet nucleation in the aqueous drop, again at midheight. The nucleated oil microdroplets are driven by Marangoni flows inside the aqueous drop and evolve into microdroplets rings. A one-dimensional multiphase and multicomponent diffusion model in combination with thermodynamical equilibrium theory is proposed to predict the behaviour of spontaneous emulsification, i.e. the microdroplet nucleation, that is triggered by diffusion. A scale analysis

*Based on: H. Tan, C. Diddens, A. Mohammed, J. Li, M. Versluis, X. Zhang, and D. Lohse, Microdroplets nucleation by dissolution of a multicomponent drop in a host liquid, Submitted.

${ }^{\dagger}$ Liquid-liquid equilibrium simulation by C. Diddens; A. Mohammed \& J. Li participated in experimental work. 
together with experimental investigations of the fluid dynamics of the system reveals that both the solutal Marangoni flow inside the drop and the buoyancydriven flow in the host liquid influence the diffusion-triggered emulsification process. Our work provides a physical understanding of the microdroplet nucleation by dissolution of a multicomponent drop in a host liquid.

\subsection{Introduction}

Multicomponent drops immersed in another liquid occur in a widespread range of engineering applications, such as chemical waste treatments, the separation of heavy metals, food processing, diagnostics and so on [118 121]. In recent years, the interest in the diffusive dynamics of multiphase fluid systems has surged, as the quantitative understanding of the process is crucial not only for fundamental studies of multiphase systems, but also for its common applications in the chemical industry, particularly for modern liquid-liquid microextraction processes [46, 122].

Diffusion processes, i.e. the movement of species down a concentration gradient, can induce a mass transfer between different phases. A classical theory about a single component bubble dissolving into a surrounding liquid was established by Epstein and Plesset [123] and later extended to drops [124] 125. It can be derived that the radial movement of the bubble surface is proportional to the square root of the time, which agrees with experimental measurements. For multicomponent drops, the situation is very different, as the consideration of the mutual interaction of the species is necessary. A consistent theory for the dissolution or growth of multicomponent drops in a host liquid was proposed by Chu and Prosperetti [17. They employed thermodynamic equilibrium constraints at the interface with the adoption of the UNIQUAC model, which is frequently applied in the description of phase equilibria. Molecular dynamics simulations were performed by Maheshwari et al. [126] with the conclusion of the importance of the interaction between the drop constituents and the host liquid during multicomponent drop dissolution. All these studies focus on the investigation of pure diffusion processes. In practice, however, the flow motion induced by the diffusion processes cannot be neglected, as the flow is able to affect the diffusion processes in turn. A small droplet, for example, can be self-propelled by Marangoni stress when the viscosity ratio of the droplet liquid to the surrounding liquid is smaller than the length scale ratio of the droplet size to the solutal interactive length scale [15]. Dietrich et al. [127] experimentally demonstrated the existence of a transition Rayleigh number for the dissolution of a sessile multicomponent drop, above which the buoyancy-driven convection in the host liquid prevails 
over the diffusion. Additionally, Dietrich et al. [128] found that diffusion is able to induce a local concentration difference and thereby cause the segregation of the components inside the drop.

For a specific category of multiphase systems with a metastable phase regime, the diffusion phenomena are more interesting and complex, as the phase equilibrium can be altered by the diffusion process, leading to the occurrence of metastable dispersions in the bulk [129]. Ouzo, an alcoholic beverage from Greece, is a typical example of this kind of solution. It mainly consists of ethanol, water and (anise) oil, and it is well mixed when the oil concentration in the solution is lower than the oil solubility of the water-ethanol solvent (aqueous phase). Spontaneous emulsification, the process of creating metastable liquid-liquid dispersions (nano- or micro-droplets), can be achieved by increasing the water concentration and thereby reducing the oil solubility without an external energy input. This is the well-known ouzo effect, which can either be triggered by simply adding water to the system or, alternatively, by the reduction of the ethanol amount by a preferential evaporation. The latter process, i.e. the evaporation of an ouzo drop, is extremely rich and exhibits multiple phase transitions during the drying, as recently discovered by Tan et al. [101].

Due to the non-uniform evaporation rates along the liquid-gas interface and the different volatilities of water and ethanol, regions of oil supersaturation, i.e. regions where the emulsification takes place, are generated locally in the evaporating ouzo drop. The same principle is expected to apply to a dissolving multicomponent drop, since it obeys the same dynamical equations and similar boundary conditions as the evaporating ouzo drop.

In this paper, we explore the dissolution of a multicomponent drop in a host liquid (Fig. 4.1), with a particular focus on systems which have the capacity of undergoing the spontaneous emulsification (ouzo effect). Experimental steps and methods are discussed in Section 4.2. The sessile drop picked here consists of the two miscible components water and ethanol with different initial ratios, and anethole oil acts as host liquid, in which ethanol is miscible and water is immiscible. The general observations and descriptions about the dissolution phenomena are given in Section 4.3. During the dissolution, we observed both spontaneous emulsifications in the region of the host oil (oil-rich phase) and in the sessile drop region (aquous phase), i.e. water-in-oil (w-in-o) microdroplets and oil-in-water (o-in-w) microdroplets correspondingly, which are presented in Section 4.4. In Section 4.5. we develop a one-dimensional diffusion model with the adoption of the so-called diffusion path theory [130] and thermodynamical equilibrium theory to provide insight to the occurrence of the spontaneous emulsification and its evolution. The so-called UNIFAC 

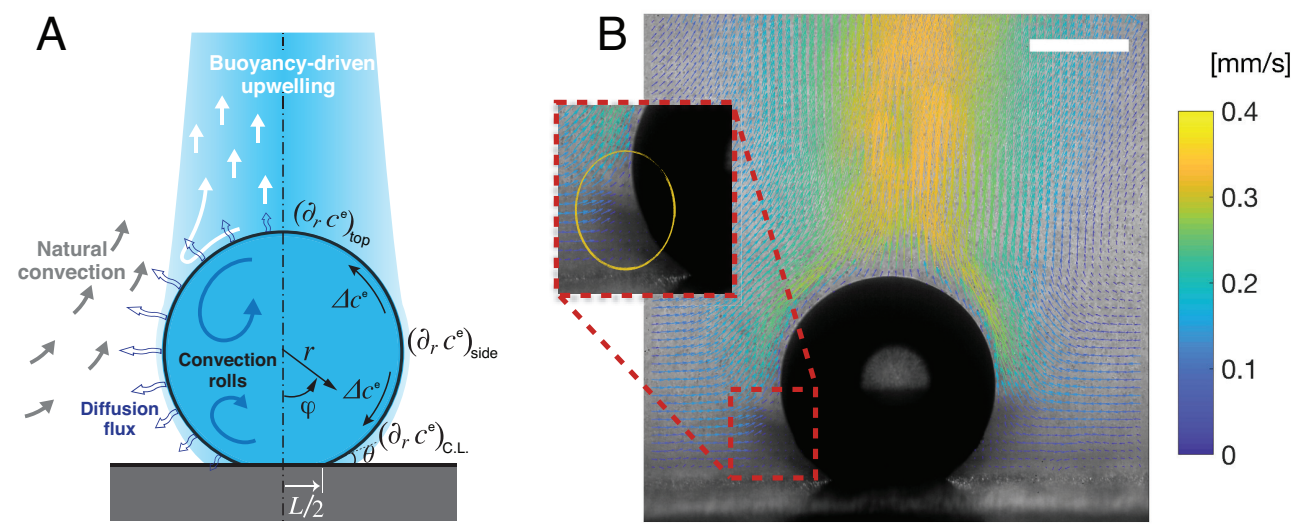

Figure 4.1: (A) Hydrodynamic sketch of a dissolving sessile drop. The left side presents the flow directions and diffusion fluxes along the drop surface. Geometrical and physical quantities are defined on the right side. (B) Experimental snapshot of the drop overlaid with the external flow field obtained by PIV-measurement. The scale bar is $0.45 \mathrm{~mm}$.

model was applied to describe phase equilibria with the consideration of liquid activity coefficients. An alternative model would be the so called UNIQUAC model used by Chu and Prosperetti [17], but the parameters of our mixture are not available for that model. Through this model, we gain insight into the emulsification process (diffusion-induced microdroplet nucleation), as well as the mass transport caused by the pure diffusion process, which are presented and evaluated in Section 4.6. To figure out the influence of flow motions on the emulsification process, a scaling analysis and micro-PIV measurements were performed in Section 4.7. The scaling analysis reveals that the Marangoni effect dominates the flow motion inside the drop, while natural convection is dominant in the host liquid (Fig. 4.1A), which was confirmed by side recording movies and micro-PIV measurement results (Fig. 4.1B). Having obtained a good understanding of the fluid dynamics in the system, we finally acquire a more systematic understanding about the dissolution process and the preferred position where the diffusion-triggered emulsification takes place.

\subsection{Experimental method}

\subsubsection{Solution and substrate}

The system we investigate here consists of Milli-Q water [obtained from a Reference A+ system (Merck Millipore) at $18.2 \mathrm{M} \Omega$ at $25^{\circ} \mathrm{C}$ ], ethanol (SigmaAldrich; $\geqslant 98 \%$ ), and anethole oil (Sigma-Aldrich; trans-anethole, $\geqslant 99.8 \%$ ). We performed dissolution experiments in a cuvette (Hellma; Inner dimensions $30 \mathrm{~mm} \times 30 \mathrm{~mm} \times 30 \mathrm{~mm}$ ), on the bottom of which a hydrophobized glass slide 
$(\approx 20 \mathrm{~mm} \times 20 \mathrm{~mm})$ was placed. A certain amount of water-saturated anethole was added into the cuvette, performing the host liquid. The depth of the liquid was $7.5 \mathrm{~mm}$. Water-ethanol binary drops with different volumetric concentrations of ethanol $(30 \%, 40 \mathrm{vol} \%, 50 \mathrm{vol} \%, 60 \mathrm{vol} \%, 70 \mathrm{vol} \%)$ were produced in the oil through a custom needle (Hamilton; outer diameter/inner diameter $0.21 \mathrm{~mm} / 0.11 \mathrm{~mm}$ ) by a motorized syringe pump (Harvard; PHD 2000), and then directly deposited on the centre of the hydrophobized glass surface.

\subsubsection{Set-up and image analysis}

The experiments were performed in a lab without people around during the data recording. The temperature of the host liquid in the cuvette was around $22^{\circ} \mathrm{C}$. The dissolution processes of the drops were observed by three synchronized cameras, one monochrome CCD camera (Ximea; MD061MU-SY) attached to a long-distance microscope system (Infinity; Model K2 DistaMax) for side view recordings, which was used for the drop profile detection, one digital SLR camera (Nikon; D750) equipped with a CMOS sensor attached to a high-magnification zoom lens system (Thorlabs; MVL12X3Z) for the other side view recordings, which was used for the side-view observation of the emulsification process, and another digital SLR camera (Nikon; D5100) equipped with a CMOS sensor attached to an identical high-magnification zoom lens system (Thorlabs; MVL12X3Z) for top view recordings, used for top-view observation of the emulsification process. A cold light source (Olympus; ILP-1) was positioned at the same side as the SLR camera to illuminate the emulsion.

We performed image analyses with a custom-made MATLAB program. The monochrome image series from the side view recordings were utilized in obtaining the temporal evolution of the dissolving characteristics of the drops. For each image, we first pre-processed the data to increase the image contrast and then calculated the profile of the drop using the Canny method [131]. The data points of the detected profile were fitted by part of a circle, i.e. assuming that the droplet is in a spherical cap shape. The diameter of drop contact area $L$ and the contact angle $\theta$ were calculated based on the geometrical relationship between the base line of the surface and the fitted spherical cap. The drop volume $V$ was calculated by integrating the volumes of the horizontal disk layers.

\subsubsection{Emulsion/microdroplets recognition}

The emulsions (nucleated microdroplets) were recognised visually. Both the water-in-oil (w-in-o) emulsion and the oil-in-water (o-in-w) emulsion had a recognisable cloudy-white appearance because of the microscopic size of the 
nucleated microdroplets, which enable them to scatter all the colours equally. The recognition of the emulsions was processed by watching the recorded videos frame by frame. Although dissolving drops were in millimeter-scale, their spherical shapes with high curvature unavoidably caused reflected light spots, which increased the difficulty of the recognition of the presence of the microdroplets inside the drop. Therefore, the presence or absence of emulsions were carefully determined by detecting the liquid colour variation and their movement from the recorded videos (both top views and synchronised side views). No fluorescence technique were used for the microdroplet detection, in order to avoid any influence of the added fluorescent materials on the spontaneous emulsification.

\subsubsection{Micro-PIV}

To investigate the flow field around the dissolving drop, we added tracking particles (Dantec dynamics; PSP-5, diam. $5 \mu \mathrm{m}$, made by nylon-12) in the host liquid at a seeding density of $0.2 \mathrm{mg} \mathrm{mL}^{-1}$ to perform micro-PIV measurements. Thanks to the low flow rate in our study, a continuous LED light source (Thorlabs; MCWHL5) was able to provide enough volume illumination for the measurements. The light source and the camera were placed at two opposite sides of the cuvette. The light passed through convex lenses before illuminating the cuvette to form a parallel light beam to increase the image contrast. At the other side, we positioned a high-speed camera [Photron Fastcam SA2 32GB, 50 frames per second(fps) at 2,048 $\times 2,048$ pixel resolution] attached with the microscope system (Infinity; Model K2 DistaMax) to perform high-speed imaging. The position of the recording system was adjusted to have a focal plane crossing the droplet centre. The thickness of the focal plane is $0.02 \mathrm{~mm}$. Thereby, we could obtain sharp images of the tracking particles within a cross-sectional plane of the drop. We took image pairs with an inter-framing time of $20 \mathrm{~ms}$ every two seconds. The obtained image pairs were first processed to reduce the noise, and then imported into PIVlab software [132, 133] to calculate the flow field. The size of interrogation window was taken as a $128 \times 128$ pixel matrix, corresponding to $142 \mu \mathrm{m} \times 142 \mu \mathrm{m}$. The interrogation window overlap was set as $75 \%$, leading to a $35.5 \mu \mathrm{m}$ vector spacing in the calculated velocity matrix.

We qualified the confidence level of the tracking particles following the flow by calculating the Stokes number and the ratio of the Stokes number to a buoyancy-corrected Froude number [134]. The relaxation time of the particle was estimated by

$$
t_{0} \equiv\left(1+\rho_{f} /\left(2 \rho_{p}\right)\right) \frac{\rho_{p} d_{p}^{2}}{18 \mu_{f}}
$$


with the consideration of added mass force [135], where $\rho_{f}=0.988 \mathrm{~g} / \mathrm{cm}^{3}$ and $\mu_{f}=4.2 \mathrm{mPa} \cdot \mathrm{s}$ are the density and the dynamic viscosity of the host liquid (trans-anethole), $\rho_{p}=1.03 \mathrm{~g} / \mathrm{cm}^{3}$ and $d_{p}=5 \mu \mathrm{m}$ the density and the diameter of the tracking particles. The Stokes number $S t \equiv t_{0} u_{\max } / R$ can be calculated as

$$
S t=\left(1+\rho_{f} /\left(2 \rho_{p}\right)\right) \frac{\rho_{p} u_{\max } d_{p}^{2}}{18 \mu_{f} R} \sim 10^{-5} \ll 1,
$$

where $u_{\max }$ is the maximum fluid velocity $\left(\sim 10 \mathrm{mms}^{-1}\right), R$ the initial radius of the drop $(\sim 0.5 \mathrm{~mm})$. The buoyancy-corrected Froude number was defined as

$$
F r \equiv \frac{u_{\max }^{2} / R}{\left(1-\rho_{f} / \rho_{p}\right) g},
$$

taking the particle density, through $\left(1-\rho_{f} / \rho_{p}\right)$, into account. With eqn. 4.2 and eqn. (4.3) we have

$$
S t / F r \sim 0.3<1,
$$

which combined with eqn. (4.2) reveals that the tracking particles exactly follow the host liquid flow surrounding the dissolving drop [134].

\subsection{Dissolution process}

\subsubsection{Characteristic states of dissolving drops}

A dissolving water-ethanol (aqueous) sessile drop in anethole oil (host liquid) is displayed in Figure 4.2. The initial ethanol concentration is $60 \mathrm{vol} \%$ and the initial drop volume is around $0.5 \mu \mathrm{L}$. The experimental snapshots (the first row are the top views and the second row are the corresponding side views) present several interesting phenomena occurring during the drop dissolution process, including an upwards rising solute plume, detaching from the top of the drop (Fig. 4.2A), spontaneous emulsifications inside and outside the drop (Fig. 4.2 BCD), and double oil-microdroplets rings forming by convection rolls and suspended in the drop (Fig. 4.2 E).

At the beginning when the drop was deposited on the hydrophobic substrate in the oil host liquid, both the drop and the surrounding oil are transparent, except for the shadows above the drop, as displayed in Figure 4.2A A. The shadow, indicated by the arrow in the figure, is the solute plume, i.e. the ethanol-rich oil mixture as ethanol diffuses into the oil surrounding the drop. They are visible thanks to the variation in light transmission when oil is mixed with ethanol. The transmission has a negative correlation with refractive index according to Beer-Lambert law. The refractive index of ethanol, 1.361, is smaller than that of trans-anethole, 1.561, and accounts for a better 

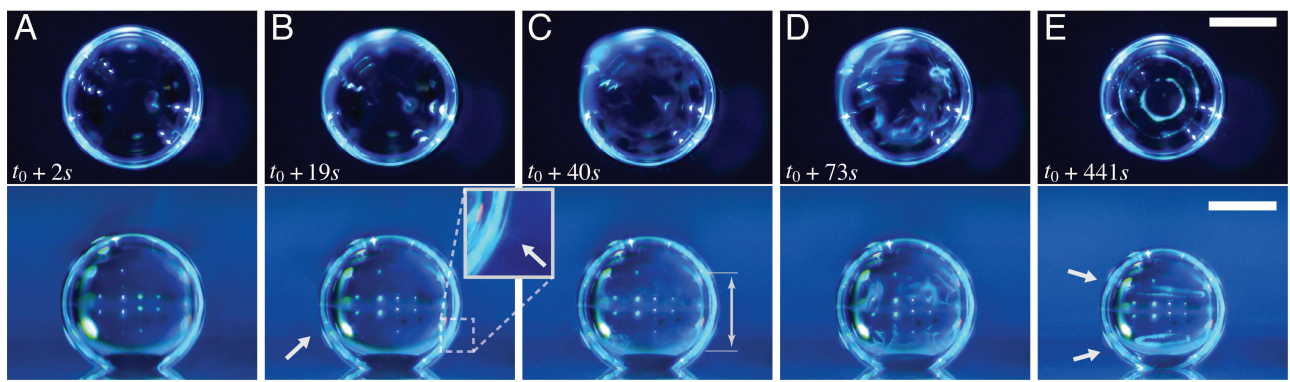

Figure 4.2: Experimental snapshots of a dissolving water/ethanol (v/v 40:60) drop in anethole oil experiencing different characteristic states during the dissolution. The first row of photos are top views and the second row are the corresponding side views. The initial drop size is around $0.5 \mu \mathrm{L}$ and $t_{0}$ denotes the drop deposition time. (A) The drop begins with a transparent appearance surrounded by a clean host liquid. The arrow indicates solute plume above the drop. (B) W-in-o emulsion (water microdroplets) suspends outside the drop. The arrows and the inserted zoom-in panel highlights the location of the microdroplets. (C) Oin-w emulsion (oil microdroplets) appears inside the drop with a preferential location around the equator of the drop (limited by the arrow). (D) More oil microdroplets are formed and concentrate at the two sides of the equator. The w-in-o emulsion disappears. (E) In around 7 minutes, the two concentrated clusters of microdroplets evolve into two rings. The scale bar is $0.5 \mathrm{~mm}$.

light transmission. In our setup, the cold light source and side-view colour camera for the emulsion observations were positioned on the same side, which indicates that the material with a better light transmission is dimming. Thus, it verifies that the solute plume is indeed an ethanol-rich oil mixture. The variation in the refractive index also causes the distortion of the light path and leads to a locally hazy scene in the top views (Fig. 4.2 ABCD).

In our system, there are two kinds of self-emulsifications: water-in-oil emulsification and oil-in-water emulsification. The former one creates water microdroplets in the oil host liquid (w-in-o emulsion) and the latter one generates oil microdroplets in the aqueous drop (o-in-w emulsion). The first appearing microdroplets are the w-in-o emulsion. They nucleate at a certain location in the surrounding oil, comparable to the position of the earth's tropic of capricorn. The emulsification normally starts within less than $5 \mathrm{~s}$ after drop deposition. Figure $4.2 \mathrm{~B}$ is a snapshot taken at $19 \mathrm{~s}$ to provide a clear visualisation of the emulsion and the inserted zoom-in panel highlights their position, indicated by arrows. The microdroplets suspend in oil for a while and then disappear. Around half a minute later, o-in-w emulsification sets in inside the drop, preferentially in the middle of the drop, concentrating in the region limited by the arrow in Figure $4.2 \mathrm{C}$. Notable, the preferential location is different from that reported in our previous work on evaporating drops, in which droplet nucleation either occurred at the contact line region for flat evaporating ouzo 
drops [101], or at the top of the drop for spherical evaporating ouzo drops [136]. More detailed discussion about those spontaneous emulsifications will be given in Section 4.4

Yet another remarkable phenomenon is that the generated o-in-w emulsions gradually form two rings of microdroplets in the drop. The generated microdroplets firstly split into two groups, one above and one below the equator of the drop, which is shown in Figure 4.2 D. The mechanism of the migration comes from two Marangoni convection rolls located separately above and below the equatorial plane. The Marangoni flow motion is induced by surface tension gradients due to concentration variations along the interface, i.e. solutal Marangoni flow. The convection rolls drive the nucleated oil microdroplets and lead to an accumulation of these in the centre of each vortex-roll, resulting in the formation of two rings of oil microdroplets as shown in Figure $4.2 \mathrm{E}$. More detailed discussion on the dynamics will be given in Section 4.7. When the ethanol in the drop has dissolved, the solute Marangoni effect stops and the rings disintegrate.

All these interesting phenomena happen during the first stage, which is mainly driven by the dissolution of ethanol from the drop. This stage takes around $30 \mathrm{~min}$. In the second stage, the remaining water diffuses with an extremely slow speed. During that stage, no further unexpected phenomena occur. Therefore, in this paper we only investigate the first stage of the process, up to the time the alcohol has fully dissolved. It is important to point out that, during the whole dissolution process, there is always a distinguishable interface between the oil host medium and the drop medium for all experiments (drops with different water-ethanol ratios) we performed. The sharp boundary of the drop corresponds to near-discontinuities in the gradient of the concentrationdistance curve [137], which reflects that the drop solution and host solution are macroscopically phase-separated at the drop-oil interface.

\subsubsection{Dissolution of drops with different initial ethanol concentrations}

To quantitively investigate the phenomena, we repeated the dissolution experiment with the drops of different initial water-ethanol ratios, from $30 \mathrm{vol} \%$ to $70 \mathrm{vol} \%$ ethanol. Figure 4.3 shows the dissolution characteristics of the drops, including the temporal evolution of the drop volume, the variations of the contact angle $\theta$ and the footprint diameter $L$. The annotations of the geometrical variables are available in a raw picture from the experiment (Fig. $4.3 \mathrm{E}$ ).

The volume evolutions of the drops are nondimensionalized by the initial drop size $V_{0}$ to demonstrate a declining trend of the residual water volume with an increasing initial ethanol concentration, as apparent from Figure $4.3 \mathrm{~A}$. The figure also reveals that indeed all drops experience two stages with two dis- 
tinguished dissolving rates, as discussed above: These two stages correspond successively to the initial stage dominated by the dissolution of the ethanol and the subsequent slow dissolution of the remaining water. This is supported by the consistence between the initial water ratios and the drop residual volume percentage after the stage transition. The same behaviour also exists in the evaporation process of multicomponent drops [57, 101]. Water has extremely small solubility (immiscible), therefore, the second stage takes an incomparably longer time than the first one (Fig. 4.3D).

The evolutions of the contact angle (Fig. $4.3 \mathrm{~B}$ ) and the footprint diameter (Fig. $4.3 \mathrm{C}$ ) also reveal the variation of the ethanol content in the drop. In first 10 to $15 \mathrm{~min}$, the contact angle increases by around 20 degrees, accompanied by a receding of the contact line. The increase of the contact angle is a result of the rising water concentration in the drop as ethanol is dissolving much faster than water. In this period, neither the constant contact radius (CR)mode nor the constant angle (CA)-mode applies [37]. In the second stage, the drop evolves nearly in CR-mode - there is a slightly decreasing contact angle with a stabilized contact area. During the whole process, the drop has a very high contact angle, more than $150^{\circ}$, because of the higher interfacial energy between the substrate and anethole compared to the energy between the substrate and the aqueous solution.

It is worth noting that, at the very beginning $(\sim 2 \mathrm{~min})$, the contact angle falls down and the footprint diameter increases by a small amount. To our best knowledge, this is the first observation of this phenomenon in dissolving multicomponent drops, although there are a few reports on this phenomenon in evaporating multicomponent drops [56, 57]. A plausible explanation is that after deposition of the drop, the ethanol molecules in the drop tend to move towards the surface because of the lower interfacial energy between the hydrophobic substrate and ethanol compared to water, which results in the decreasing contact angle and the increasing contact area size [57].

\subsection{Spontaneous emulsification}

As stated above, during the dissolution, spontaneous emulsification happens both in the host oil and in the drop, forming w-in-o emulsions and o-in-w emulsions successively. The former ones are nucleated water microdroplets suspending in anethole oil, whereas the latter ones are anethole oil microdroplets nucleated in the aqueous phase. We performed 24 groups of dissolution experiments with five different water-to-ethanol ratios as the drop initial composition to study the impact of the composition on the emulsification phenomena. In Table 4.1, the observed emulsification behaviours for the different 

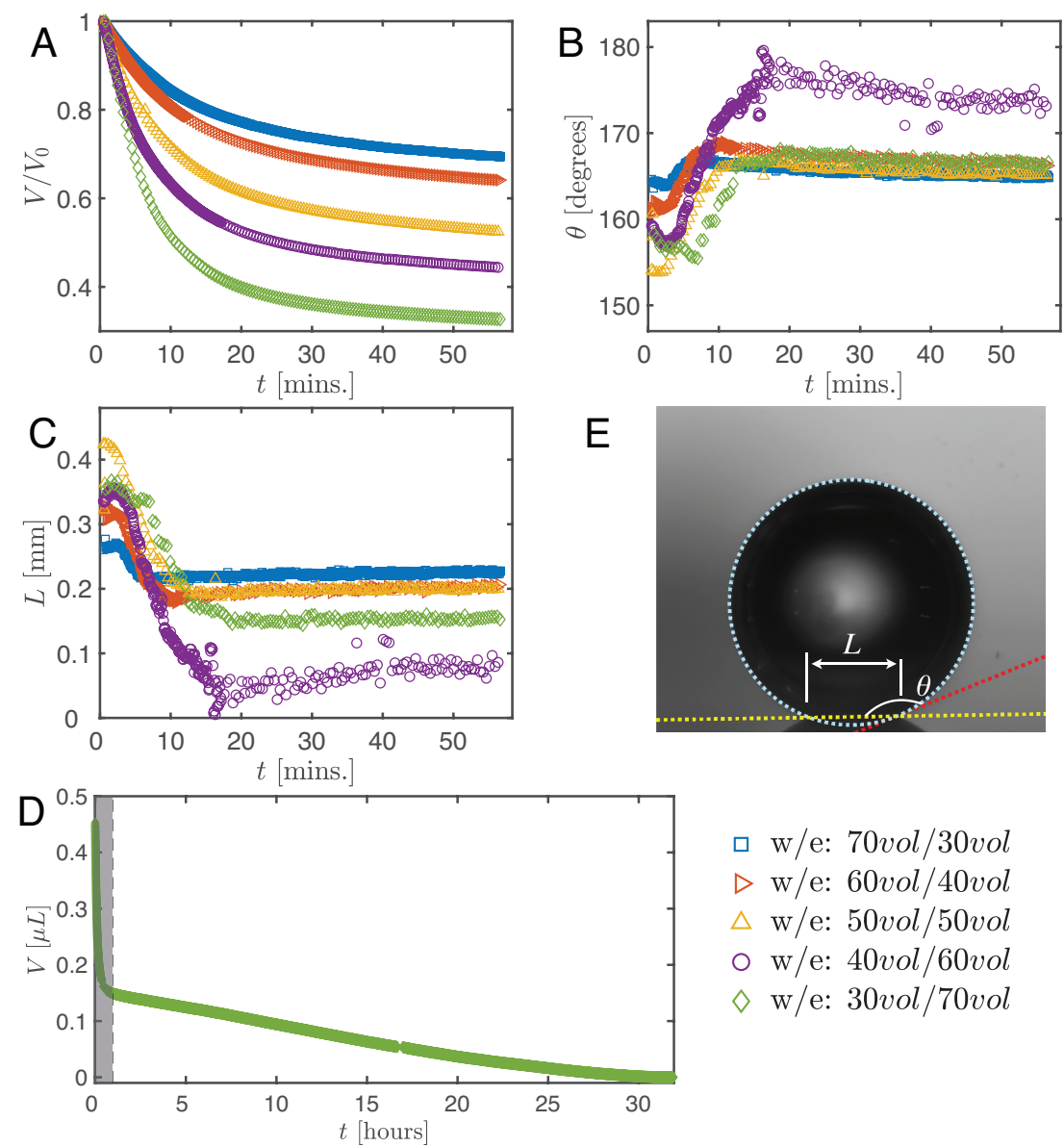

$\square \mathrm{w} / \mathrm{e}: 70 \mathrm{vol} / 30 \mathrm{vol}$

$\triangleright \mathrm{w} / \mathrm{e}: 60 \mathrm{vol} / 40 \mathrm{vol}$

$\triangle \mathrm{w} / \mathrm{e}: 50 \mathrm{vol} / 50 \mathrm{vol}$

○ w/e: $40 \mathrm{vol} / 60 \mathrm{vol}$

$\diamond \mathrm{w} / \mathrm{e}: 30 \mathrm{vol} / 70 \mathrm{vol}$

Figure 4.3: Morphology evolution of dissolving drops created with five different initial waterto-ethanol ratios (v/v 30:70, 40:60, 50:50, 60:40, 70:30). (A-C) present temporal evolutions of the nondimensionalized volume $V / V_{0}$ by initial drop size $V_{0}$, contact angle $\theta$, and footprint diameter $L$, respectively, during the dissolution. For one case (v/v 30:70) the volume evolution of the whole dissolution process is displayed in (D). The sheded area on the left is shown in (A). In (E), a recording image of a dissolving drop is shown with annotations of the geometrical parameters. 
A water microdroplets nucleation outside drop (w-in-o):

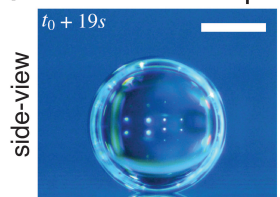

(30 vol\% EtOH)

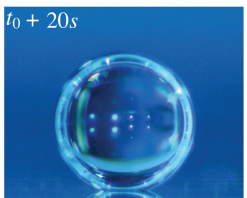

(40 vol\% EtOH)

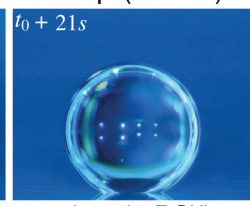

(50 vol\% EtOH)

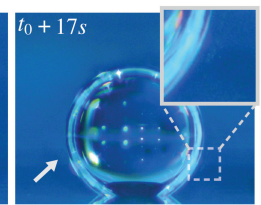

(60 vol\% EtOH)

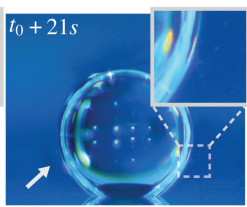

(70 vol\% EtOH)

B oil microdroplets nucleation inside drop (o-in-w):
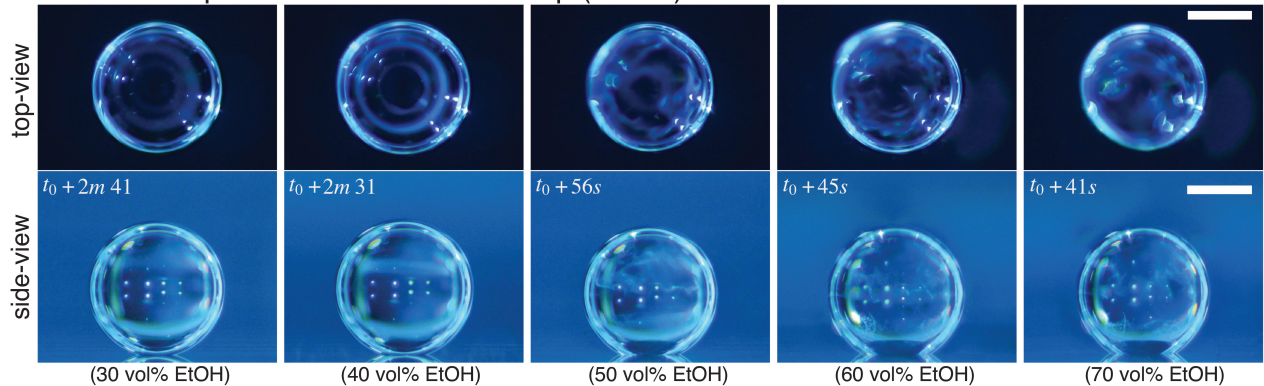

(70 vol\% EtOH)

Figure 4.4: Experimental snapshots of the w-in-o emulsification (A) and the o-in-w emulsification (B) of five different dissolving water/ethanol drops with different initial water-toethanol ratios (v/v 30:70, 40:60, 50:50, 60:40, 70:30). The side-view photographs in A show that water-in-oil emulsification only happens for the high initial ethanol concentration cases ( $60 \mathrm{vol} \%$ and $70 \mathrm{vol} \%$ ). The synchronized side- and top-view photographs in B display o-in-w emulsification occurring in all the cases. The scale bars are $0.5 \mathrm{~mm}$.

initial drop compositions are listed. $\mathrm{Y} / \mathrm{N}$ stands for presence/absence of the emulsification. The waiting time for the onset of emulsification, measured with respect to the moment of needle detachment from the drop, are given in parentheses. W-in-o emulsification occurs only when initial ethanol content in drop is high $(\geq 50 \mathrm{vol} \%)$ and its onset time is very short, within seconds. On the contrary, o-in-w emulsification occurs for all the cases, independent of the ethanol content, but more than half a minute later. The corresponding onset time has a negative correlation with the initial ethanol content in the drop.

Figure 4.4 shows photographs of the emulsion detection experiments. In Figure 4.4, cloudy-white w-in-o emulsions (nucleated water microdroplets) appear and suspend outside the drop, when the drop is created with $60 \%$ and $70 \%$ volume percentage ethanol. While for the $30 \mathrm{vol} \%$ and $40 \mathrm{vol} \%$ cases, the surrounding anethole oil remains clean - there is no w-in-o emulsions. $50 \mathrm{vol} \%$ is apparently the transition point, as for this case, in some experiments the win-o emulsions appear and in others they do not. As stated above, in all of our experiments, we found that the emulsions only appear at a certain location, close to the tropic of capricorn of the drop and in the vicinity of the drop interface, as pointed out by arrows in the inserted pictures. The appeared emulsion move up and down at this location and some microdroplets are driven away by natural convection in the host liquid (cf. section 4.7). After around half 
a minute, the w-in-o emulsion disappears and the surrounding liquid becomes transparent again.

The o-in-w emulsion (nucleated oil microdroplets) in the drop shows up independently of the initial ethanol concentration of the drop. The cloudywhite o-in-w emulsions inside the drop are visible in all cases, as displayed in Figure $4.4 \mathrm{~B}$. The oil microdroplets emerge near the equator of the drop and then follow the Marangoni flow. Gradually, the oil microdroplets concentrate at the centre of the convection rolls and form two microdroplets rings. We provide top view photographs (second row of Fig. 4.4 B) for each case, from which the rings are clearly visible. The first two columns of photographs show two already formed rings of oil microdroplets, whereas the last three columns show the early chaotic arrangement of the microdroplets before the rings formed.

\subsection{One-dimensional multicomponent diffusion model}

More quantitative insight into the spontaneous emulsification is gained by theoretically analysing the multi-diffusion process of the water-ethanol drop dissolving in the host liquid. A pure diffusion model is developed for the early stage of the diffusion process, which, together with the so-called diffusion path method [130, 138], is used to predict the absence or appearance of emulsification. Our model consists of two parts, namely one modelling the mass transport in the two multicomponent fluids in contact (Sec. 4.5.1) and the other one calculating the liquid-liquid equilibrium at the interface of the two regions (Sec. 4.5.2). The mass transport is modelled as a one-dimensional problem with a moving interface separating the aqueous phase and the oilrich phase, known as Stefan problem [18. The liquid-liquid equilibrium of the ternary mixture is calculated by applying the condition of equal chemical potentials (fundamental thermodynamic relation) in combination with the UNIFAC model to quantify the nonideality of the mixture.

Mass transport in liquids happens on a large time scale compared to the macroscopic phase separation at the interface, and therefore these two processes are decoupled in our model. To decouple those two processes, the following assumptions were made: We assume that the equilibrium at the interface is instantaneously achieved and remains stable during the mass transport across the interface. To further simplify the model, we also assume zero boundary thickness, i.e. disregarding the microscopic details of the thermodynamic equilibrium process. With these assumptions, the interfacial composition is directly given by the liquid-liquid equilibrium calculation and thereby determines the mass transport model. 


\begin{tabular}{|c|c|c|c|c|c|}
\hline \multirow{2}{*}{$\begin{array}{c}\text { drop } \\
\text { solution } \\
\text { w:e } \\
\end{array}$} & \multirow[b]{2}{*}{ trial } & \multicolumn{4}{|c|}{ spontaneous emulsification } \\
\hline & & w-in-o & $\begin{array}{c}\text { onset } \\
\text { time }\end{array}$ & o-in-w & $\begin{array}{c}\text { onset } \\
\text { time }\end{array}$ \\
\hline \multirow{4}{*}{$\begin{array}{c}7: 3 \\
(\mathrm{v} / \mathrm{v})\end{array}$} & 1 & $\mathrm{~N}$ & - & Y & $69.0 \mathrm{~s}$ \\
\hline & 2 & $\mathrm{~N}$ & - & Y & $81.5 \mathrm{~s}$ \\
\hline & 3 & $\mathrm{~N}$ & - & $\mathrm{Y}$ & $62.0 \mathrm{~s}$ \\
\hline & 4 & $\mathrm{~N}$ & - & Y & $69.5 \mathrm{~s}$ \\
\hline \multirow{4}{*}{$\begin{array}{c}6: 4 \\
(\mathrm{v} / \mathrm{v})\end{array}$} & 1 & $\mathrm{~N}$ & - & Y & $57.5 \mathrm{~s}$ \\
\hline & 2 & $\mathrm{~N}$ & - & $\mathrm{Y}$ & $70.0 \mathrm{~s}$ \\
\hline & 3 & $\mathrm{~N}$ & - & Y & $61.0 \mathrm{~s}$ \\
\hline & 4 & $\mathrm{~N}$ & - & $\mathrm{Y}$ & $62.5 \mathrm{~s}$ \\
\hline \multirow{5}{*}{$\begin{array}{c}5: 5 \\
(\mathrm{v} / \mathrm{v})\end{array}$} & 1 & Y & $5.0 \mathrm{~s}$ & Y & $27.0 \mathrm{~s}$ \\
\hline & 2 & $\mathrm{~N}$ & - & Y & $33.0 \mathrm{~s}$ \\
\hline & 3 & Y & $5.0 \mathrm{~s}$ & Y & $32.5 \mathrm{~s}$ \\
\hline & 4 & $\mathrm{Y}$ & $3.0 \mathrm{~s}$ & $\mathrm{Y}$ & $36.0 \mathrm{~s}$ \\
\hline & 5 & Y & $2.0 \mathrm{~s}$ & $\mathrm{Y}$ & $28.0 \mathrm{~s}$ \\
\hline \multirow{4}{*}{$\begin{array}{c}4: 6 \\
(\mathrm{v} / \mathrm{v})\end{array}$} & 1 & Y & $5.0 \mathrm{~s}$ & Y & $26.0 \mathrm{~s}$ \\
\hline & 2 & $\mathrm{Y}$ & $3.5 \mathrm{~s}$ & Y & $29.0 \mathrm{~s}$ \\
\hline & 3 & $\mathrm{Y}$ & $1.5 \mathrm{~s}$ & Y & $29.5 \mathrm{~s}$ \\
\hline & 4 & Y & $3.0 \mathrm{~s}$ & Y & $39.5 \mathrm{~s}$ \\
\hline \multirow{7}{*}{$\begin{array}{c}3: 7 \\
(\mathrm{v} / \mathrm{v})\end{array}$} & 1 & $\mathrm{Y}$ & $4.0 \mathrm{~s}$ & Y & $28.0 \mathrm{~s}$ \\
\hline & 2 & Y & $3.0 \mathrm{~s}$ & Y & $30.0 \mathrm{~s}$ \\
\hline & 3 & $\mathrm{Y}$ & $3.5 \mathrm{~s}$ & $\mathrm{Y}$ & $30.0 \mathrm{~s}$ \\
\hline & 4 & $\mathrm{Y}$ & $5.0 \mathrm{~s}$ & $\mathrm{Y}$ & $29.0 \mathrm{~s}$ \\
\hline & 5 & $\mathrm{Y}$ & $2.0 \mathrm{~s}$ & $\mathrm{Y}$ & $25.5 \mathrm{~s}$ \\
\hline & 6 & $\mathrm{Y}$ & $3.0 \mathrm{~s}$ & $\mathrm{Y}$ & $29.5 \mathrm{~s}$ \\
\hline & 7 & $\mathrm{Y}$ & $1.0 \mathrm{~s}$ & Y & $34.5 \mathrm{~s}$ \\
\hline
\end{tabular}

Table 4.1: Spontaneous emulsifications by dissolution of multicomponent drops with different initial compositions in the host liquid. The subindices e, w, and o denote components ethanol, water, and anethole oil, respectively. $\mathrm{Y}$ and $\mathrm{N}$ stands for presence and absence of the emulsification, respectively. The time values are onset time of emulsification. 
Stefan problems commonly exists in many studies involving diffusion, such as heat transfer with a phase transition (thawing, freezing, melting), moisture transport of swelling grains or polymers [139]. Classic solutions to Stefan problems are given by Crank [18]. In subsection 4.5.1, we present definitions and the derivation of the equations with respect to our problem.

The establishment of the liquid-liquid equilibria between two phases happens on a short time scale compared to the diffusion process in the adjacent bulk regions. Once the aqueous medium and the host liquid are in contact, thermodynamic equilibrium favours an oil-rich phase on one side coexisting with a water-rich phase on the other side, i.e. a macroscopic phase separation, which is observed as the sharp boundary of the water-ethanol drop in oil. The composition of these two phases are situated on the binodal curve (or coexistence curve) in the ternary phase diagram of the system and are connected by a tie line. A brief description of the applied equilibrium theory is given in subsection 4.5.2,

\subsubsection{Mass transport}

Our system is composed of three species in two different phases, i.e. it is a multicomponent and multiphase diffusion system. The diffusion process is modelled in a one-dimensional infinite space, as illustrated in Figure 4.5. The diffusion process of each individual constituent is assumed to depend only on its own concentration gradient in radial direction (Fick's diffusion laws). $m_{\alpha}$ denotes the mass fraction of the species water $(\alpha=\mathrm{w})$, ethanol $(\alpha=\mathrm{e})$ and anethole oil $(\alpha=0)$ as function of time $t$ and position $x$. The superscripts indicate the region, i.e. either drop region (d) or host liquid region (h). The two regions are separated by the moving interface at position $s(t)$. The initial position of the interface is set to the origin, i.e. $s(0)=0$. The mass transport of each component $\alpha$ is formulated by a system of equations

$$
\begin{aligned}
\frac{\partial m_{\alpha}^{\mathrm{d}}}{\partial t} & =D_{\alpha}^{\mathrm{d}} \frac{\partial^{2} m_{\alpha}^{\mathrm{d}}}{\partial x^{2}} & & -\infty<x \leq s(t), \\
\frac{\partial m_{\alpha}^{\mathrm{h}}}{\partial t} & =D_{\alpha}^{\mathrm{h}} \frac{\partial^{2} m_{\alpha}^{\mathrm{h}}}{\partial x^{2}} & & s(t) \leq x<+\infty, \\
\left(m_{\alpha}^{\mathrm{h}}-m_{\alpha}^{\mathrm{d}}\right) \frac{\mathrm{d} s}{\mathrm{~d} t} & =D_{\alpha}^{\mathrm{d}} \frac{\partial m_{\alpha}^{\mathrm{d}}}{\partial x}-D_{\alpha}^{\mathrm{h}} \frac{\partial m_{\alpha}^{\mathrm{h}}}{\partial x} & & \text { when } \quad x=s(t),
\end{aligned}
$$

where the first equation governs the diffusion process in the drop region with a corresponding diffusivity $D_{\alpha}^{\mathrm{d}}$, and the second one for the host liquid with diffusivity $D_{\alpha}^{\mathrm{h}}$. The Stefan condition is considered in the third equation, implying mass balances at the interface between the change rate of local mass due to the interface movement (lhs) and the combined diffusive mass flux of 


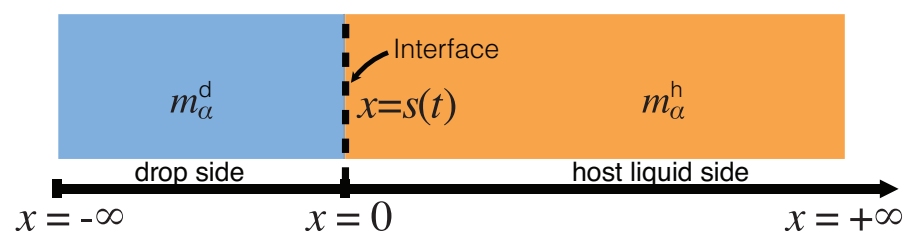

Figure 4.5: Sketch of the one-dimensional multiphase and multicomponent diffusion model with a moving interface at $x=s(t)$ separating the two regions of drop (d) and host liquid (h). The subscript $\alpha$ stands for the species water (w) and anethole oil (o). The mass fractions of the different species in the two different regions $m_{\alpha}^{\mathrm{d}}$ and $m_{\alpha}^{\mathrm{h}}$ are function of time $t$ and position $x$. The initial position of interface $s(0)$ is defined to be at the origin.

the species at the interface (rhs). The composition in both phases is subject to the constraints

$$
\begin{aligned}
& m_{\mathrm{w}}^{\mathrm{d}}+m_{\mathrm{e}}^{\mathrm{d}}+m_{\mathrm{o}}^{\mathrm{d}}=1, \\
& m_{\mathrm{w}}^{\mathrm{h}}+m_{\mathrm{e}}^{\mathrm{h}}+m_{\mathrm{o}}^{\mathrm{h}}=1 .
\end{aligned}
$$

Therefore, only two of three species, for instance water $(\alpha=\mathrm{w})$ and anethole oil $(\alpha=\mathrm{o})$, are solved by equations (4.5), and the third one, ethanol $(\alpha=\mathrm{e})$, is calculated by this relationship.

As we only focus on the early stage of the diffusion process, the boundaries at $\pm \infty$ do not influence the dynamics in the vicinity of the interface. Therefore, the initial condition and the far field boundary condtitions are given by

$$
\begin{array}{lr}
m_{\alpha}^{\mathrm{d}}=m_{\alpha 0}^{\mathrm{d}}, & -\infty<x<0, t=0, \\
m_{\alpha}^{\mathrm{h}}=m_{\alpha 0}^{\mathrm{h}}, & 0<x<\infty, t=0, \\
m_{\alpha}^{\mathrm{d}}=m_{\alpha 0}^{\mathrm{d}}, & x=-\infty, t>0, \\
m_{\alpha}^{\mathrm{h}}=m_{\alpha 0}^{\mathrm{h}}, & x=\infty, t>0 .
\end{array}
$$

The initial values are input from experimental conditions, as we know the initial composition of the drop mixture and the host liquid. Denoting the interfacial compositions at the drop and the host liquid side of the interface as $m_{\alpha s}^{\mathrm{d}}$ and $m_{\alpha s}^{\mathrm{h}}$, respectively, we have

$$
\begin{array}{ll}
m_{\alpha}^{\mathrm{d}}=m_{\alpha s}^{\mathrm{d}}, & x=s(t), t>0 \\
m_{\alpha}^{\mathrm{h}}=m_{\alpha s}^{\mathrm{h}}, & x=s(t), t>0 .
\end{array}
$$

The interfacial composition on the two sides of the interface are calculated by applying the liquid-liquid equilibrium constraint at the interface condition, cf. Sec. 4.5.2. In a ternary system, the interfacial composition has four degrees of freedom by virtue of mass conservation of the mixture. The composition on 
two sides of the interface are situated on the binodal and are connected by a tie line in the phase diagram of the ternary system. Therefore, in accordance with Gibbs' phase rule, the number of degrees of freedom for the interfacial composition reduces to one (for instance $m_{\mathrm{ws}}^{\mathrm{d}}$ ).

The solution of the governing diffusion equations 4.5 with the given conditions (4.7) reads

$$
\begin{aligned}
& m_{\alpha}^{\mathrm{d}}=\mathcal{B}_{\alpha}^{1}+\mathcal{B}_{\alpha}^{2} \operatorname{erf} \frac{x}{2 \sqrt{D_{\alpha}^{\mathrm{d}} t}}, \\
& m_{\alpha}^{\mathrm{h}}=\mathcal{B}_{\alpha}^{3}+\mathcal{B}_{\alpha}^{4} \operatorname{erf} \frac{x}{2 \sqrt{D_{\alpha}^{\mathrm{h}} t}}
\end{aligned}
$$

where $\mathcal{B}_{\alpha}^{1}, \mathcal{B}_{\alpha}^{2}, \mathcal{B}_{\alpha}^{3}$ and $\mathcal{B}_{\alpha}^{4}$ are coefficients to be determined. By applying the additional conditions (4.8) to the solution form, we obtain the coefficients

$$
\begin{aligned}
\mathcal{B}_{\alpha}^{1}\left(\frac{s}{\sqrt{t}}\right) & =\frac{m_{\alpha 0}^{\mathrm{d}} \operatorname{erf} \frac{s}{2 \sqrt{D_{\alpha}^{\mathrm{d}} t}}+m_{\alpha s}^{\mathrm{d}}}{\operatorname{erf} \frac{s}{2 \sqrt{D_{\alpha}^{\mathrm{d}} t}}+1} \\
\mathcal{B}_{\alpha}^{2}\left(\frac{s}{\sqrt{t}}\right) & =\frac{m_{\alpha s}^{\mathrm{d}}-m_{\alpha 0}^{\mathrm{d}}}{\operatorname{erf} \frac{s}{2 \sqrt{D_{\alpha}^{\mathrm{d}} t}}+1}, \\
\mathcal{B}_{\alpha}^{3}\left(\frac{s}{\sqrt{t}}\right) & =\frac{m_{\alpha 0}^{\mathrm{h}} \operatorname{erf} \frac{s}{2 \sqrt{D_{\alpha}^{\mathrm{h}} t}}-m_{\alpha s}^{\mathrm{h}}}{\operatorname{erf} \frac{s}{2 \sqrt{D_{\alpha}^{\mathrm{h}} t}}-1} \\
\mathcal{B}_{\alpha}^{4}\left(\frac{s}{\sqrt{t}}\right) & =\frac{m_{\alpha s}^{\mathrm{h}}-m_{\alpha 0}^{\mathrm{h}}}{\operatorname{erf} \frac{s}{2 \sqrt{D_{\alpha}^{\mathrm{h}} t}}-1}
\end{aligned}
$$

which are function of $s / \sqrt{t}$. Following the way successfully used by previous researchers [130, 138, 140], we assume the movement to be proportional to $\sqrt{t}$, which is consistent with the fact that the interface movement is driven by diffusion, i.e.

$$
s(t)=\lambda \sqrt{t}, \quad \text { and } \sqrt{D_{s}}=|\lambda|,
$$

where the sign of the constant $\lambda$ gives the direction of the interface movement, and $D_{s}$ can be regarded as the "diffusion coefficient" of the moving interface. Thus, the undetermined coefficients are function of $\lambda$.

To simplify the expression of the equations, we introduce the definitions

$$
\mathcal{K}_{\alpha}=\frac{\lambda}{2 \sqrt{D_{\alpha}^{\mathrm{h}}}}, \quad \mathcal{R}_{\alpha}=\sqrt{\frac{D_{\alpha}^{\mathrm{h}}}{D_{\alpha}^{\mathrm{d}}}}, \quad \chi_{\mathrm{o}}^{\mathrm{w}}=\sqrt{\frac{D_{\mathrm{o}}^{\mathrm{h}}}{D_{\mathrm{w}}^{\mathrm{h}}}},
$$


which together with equation 4.11) are substituted into the coefficients 4.10) and the Stefan condition (4.5). Thereby, we obtain the coefficient constants

$$
\begin{aligned}
\mathcal{B}_{\alpha}^{1} & =\frac{m_{\alpha 0}^{\mathrm{d}} \operatorname{erf}\left(\mathcal{R}_{\alpha} \mathcal{K}_{\alpha}\right)+m_{\alpha s}^{\mathrm{d}}}{\operatorname{erf}\left(\mathcal{R}_{\alpha} \mathcal{K}_{\alpha}\right)+1}, \\
\mathcal{B}_{\alpha}^{2} & =\frac{m_{\alpha s}^{\mathrm{d}}-m_{\alpha 0}^{\mathrm{d}}}{\operatorname{erf}\left(\mathcal{R}_{\alpha} \mathcal{K}_{\alpha}\right)+1}, \\
\mathcal{B}_{\alpha}^{3} & =\frac{m_{\alpha 0}^{\mathrm{h}} \operatorname{erf} \mathcal{K}_{\alpha}-m_{\alpha s}^{\mathrm{h}}}{\operatorname{erf} \mathcal{K}_{\alpha}-1}, \\
\mathcal{B}_{\alpha}^{4} & =\frac{m_{\alpha s}^{\mathrm{h}}-m_{\alpha 0}^{\mathrm{h}}}{\operatorname{erf} \mathcal{K}_{\alpha}-1},
\end{aligned}
$$

as well as the new expression of mass conservation 4.5c)

$$
\sqrt{\pi} \mathcal{K}_{\alpha}\left(m_{\alpha s}^{\mathrm{h}}-m_{\alpha s}^{\mathrm{d}}\right)=4 e^{-\mathcal{K}_{\alpha}^{2}}\left[-\mathcal{B}_{\alpha}^{4}+e^{\mathcal{K}_{\alpha}^{2}\left(1-\mathcal{R}_{\alpha}^{2}\right)} \mathcal{B}_{\alpha}^{2} / \mathcal{R}_{\alpha}\right] .
$$

We thus have a closed-form solution for the problem, given by the mass balance equation (4.14) above in adjunction with thermodynamic equilibria theory. The number of the mass balance equations is two, one for water $(\alpha=\mathrm{w})$ and the other for anethole oil $(\alpha=\mathrm{o})$ and the five unknown variables are $m_{\mathrm{w} s}^{\mathrm{d}}, \mathrm{d} m_{\mathrm{o} s}^{\mathrm{d}}, m_{\mathrm{w} s}^{\mathrm{h}}, m_{\mathrm{os}}^{\mathrm{h}}$, and $\lambda$. As discussed above, the composition on two sides of the interface are situated on the binodal and are connected by a tie line in the phase diagram. Due to these equilibrium constraints, the number of the freedom degrees of interfacial composition is one (for instance $m_{\mathrm{w} s}^{\mathrm{d}}$ ). Hence, there are in total two unknown variables, namely $m_{\mathrm{w} s}^{\mathrm{d}}$ and $\lambda$, in the equation. By applying Newton's method with a given initial guess, we can find roots of equations (4.14) and the whole problem is solved. All quantities defined in the model are list in Table 4.2

\subsubsection{Liquid-liquid equilibrium at interface}

The liquid-liquid equilibrium is achieved when the chemical potentials $\mu_{\alpha}$ are the same in both phases for each species $\alpha$. The chemical potential is function of pressure, temperature, and mole fractions $x_{\alpha}$ of the liquid constituents. At fixed temperature and pressure, a three component liquid-liquid equilibrium system only has a single degree of freedom [17]. The condition of equality of the chemical potentials then reduces to

$$
x_{\alpha}^{\mathrm{d}} \gamma_{\alpha}^{\mathrm{d}}=x_{\alpha}^{\mathrm{h}} \gamma_{\alpha}^{\mathrm{h}},
$$

where $\gamma$ is the liquid activity coefficient, a correction factor accounting for the nonideality of the mixture. Subscripts and superscripts here have the same meaning as in the previous section. 

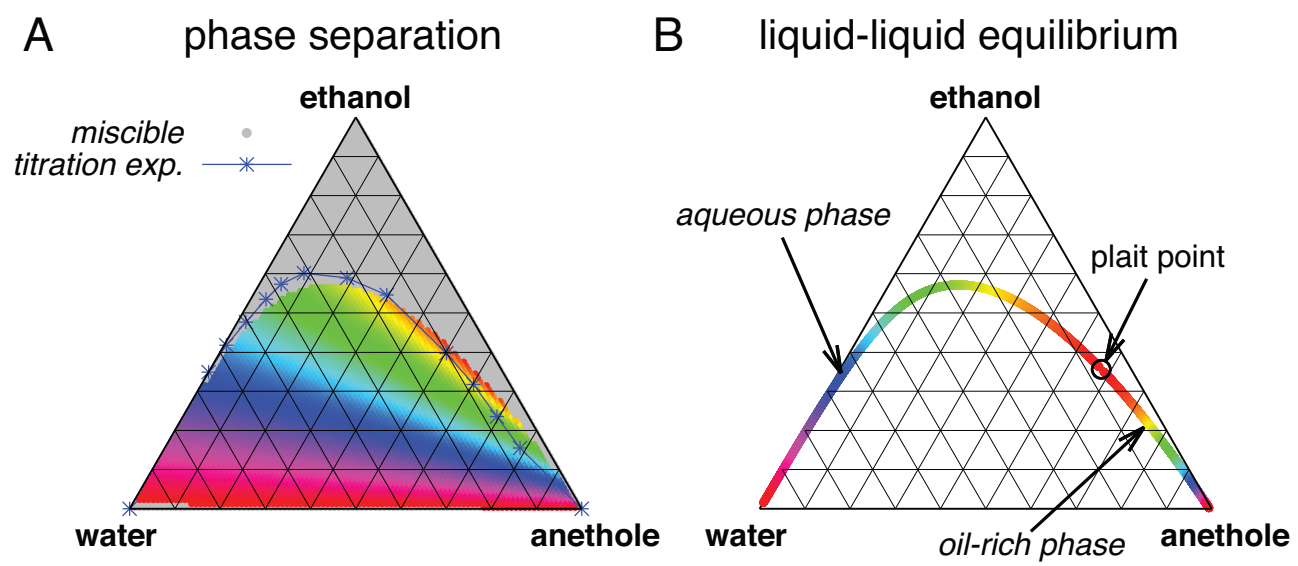

Figure 4.6: Phase separation predicted by UNIFAC. (A) gray regions indicated homogenoeus mixing, whereas phase separation is expected in the coloured region. The blue line indicates the good agreement with the titration experiments of Tan et al. [136]. If the liquid is undergoing phase separation, the composition of the resulting two phases can be read off from the binodal in (B) by the color code.

Obviously, the mixture can only undergo phase separation if the mixture is nonideal, i.e. $\gamma_{\alpha} \neq 1$. Thus, the activity coefficients are fundamental to accurately describe the liquid-liquid equilibrium. Unfortunately, we have neither found sufficiently detailed experimental data for the present mixture, nor parameters for the UNIQUAC model, which was used by Chu and Prosperetti [17]. Therefore, we employed the UNIFAC model [141], which is more general than the UNIQUAC model, to perform the splitting of the molecules into functional subgroups. For detailed information about this model, we refer to the reference [142]. Due to the better agreement with the titration experiments by Tan et al. [136], we took the recent modified UNIFAC (Dortmund) parametrization [143].

In order to find the liquid-liquid equilibrium curve and the regions of phase separation, we followed the method propsed by Zuend et al. [144]: if equations 4.15 can only be solved trivially, i.e. $x_{\alpha}^{\mathrm{d}}=x_{\alpha}^{\mathrm{h}}$, or if the Gibbs free energy at the trivial solution is below the Gibbs free energy of all non-trivial solutions, the mixture remains in a well-mixed single phase configuration. On the contrary, if there is a non-trivial solution with a lower Gibbs free energy than the trivial solution, phase separation is expected and the system will take on the solution with the globally minimal Gibbs free energy.

The data obtained by this procedure is depicted in Figure 4.6. In Figure 4.6. A, the region of phase separation is shown in a ternary diagram in terms of mass fractions $m_{\alpha}$. In gray regions, the liquid at the specific composition remains perfectly mixed, whereas in the coloured regions, phase sepearation 
occurs. The composition of the two resulting phases can be read off from Figure $4.6 \mathrm{~B}$ by the color code: The system splits into an aqueous phase and an oil-rich phase which compositions are indicated by the same color. Although the UNIFAC model is in general not perfect due to its dependence on the parameter table, the comparison of the phase separation region with the titration data of Tan et al. [101] in Figure 4.6A show good agreement.

\subsubsection{Diffusion coefficients}

In the model, there are in total four different diffusion coefficients, namely in each of the two different regions one coefficient for one of the two species: water diffusivity in the drop medium $D_{\mathrm{w}}^{\mathrm{d}}$, anethole oil diffusivity in the drop medium $D_{\mathrm{o}}^{\mathrm{d}}$, water diffusivity in the host liquid medium $D_{\mathrm{w}}^{\mathrm{h}}$, and anethole oil diffusivity in the host liquid medium $D_{\mathrm{o}}^{\mathrm{h}}$.

To acquire the diffusivities, we were confined to model predictions, since the direct measurement is complicated. In the drop medium at the early stage, the trans-anethole content is negligible, so that the coefficient $D_{\mathrm{w}}^{\mathrm{d}}$ is assumed to be given by the mutual diffusivity in a binary water-ethanol mixture. The corresponding values were obtained by fitting the experimental data in literature [69]. The coefficient $D_{\mathrm{o}}^{\mathrm{d}}$, i.e. dilute anethole in a water/ethanol mixture, is calculated in the limit of infinite dilution based on the model of Perkins and Geankoplis [145].

Both $D_{\mathrm{w}}^{\mathrm{d}}$ and $D_{\mathrm{o}}^{\mathrm{d}}$ depend on the initial ethanol content in drop. The model of Hayduk and Minhas [146] was used to calculate the coefficients of dilute water in host anethole oil $D_{\mathrm{w}}^{\mathrm{h}}$. The corresponding diffusivity constant $D_{\mathrm{o}}^{\mathrm{h}}$ is estimated according to the Stokes-Einstein equation, which states diffusion coefficient is inversely proportional to the size of molecules at constant viscosity. Since both ethanol and water content in the host liquid are negligible, we take $D_{\mathrm{w}}^{\mathrm{h}}=1.2 \times 10^{-9} \mathrm{~m}^{2} / \mathrm{s}$ and $D_{\mathrm{o}}^{\mathrm{h}}=0.66 \times 10^{-9} \mathrm{~m}^{2} / \mathrm{s}$, both of which are assumed to be constant.

\subsection{Model predictions}

\subsubsection{Concentration profiles}

The mass concentration distribution of each constituent as function of time is obtained from the model described above. Figure 4.7 ABC and corresponding close-up images, Figure 4.7DE, show the development of the concentration profiles for different cases of binary drops with different initial water-ethanol ratios $(70: 30 \mathrm{v} / \mathrm{v}$ in $\mathrm{A}, 50: 50 \mathrm{v} / \mathrm{v}$ in $\mathrm{B}$, and $30: 70 \mathrm{v} / \mathrm{v}$ in $\mathrm{C})$. Anethole oil, water, and ethanol are labeled with yellow, blue, and red colours, respectively. 

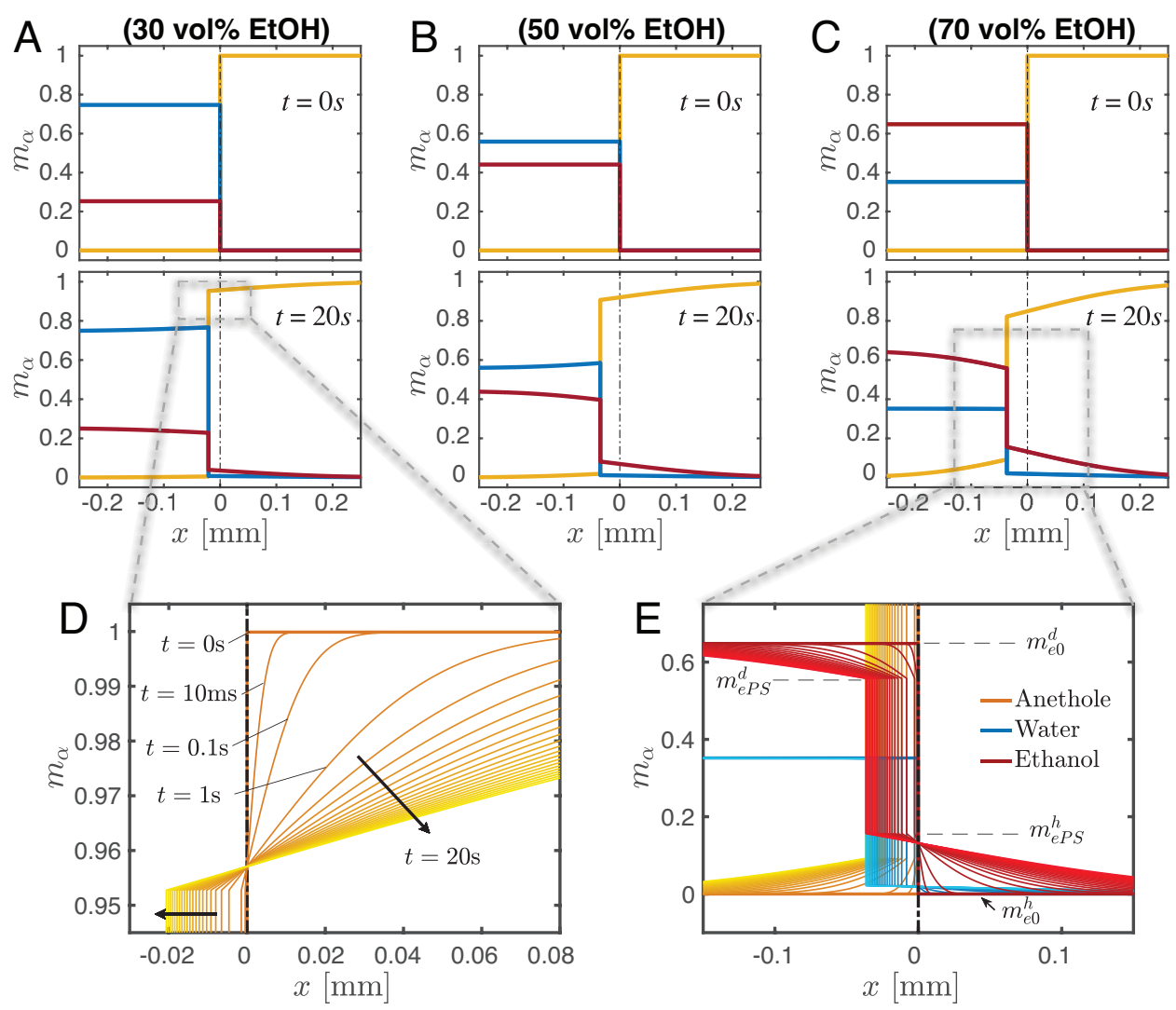

Figure 4.7: Calculated results of the diffusion model using the parameters described in the text. (A-C) Snapshots of the mass fraction profile at 0s (first row) and 20s (second row) for different cases. The vertical black dash-dotted line indicates the initial position of the interface, which separates the drop solution on the left side of figures and the host liquid on the right side. Panels D and E are zoom-in pictures of panels A and C, showing the profile evolution.

The vertical black dashed line stands for the initial position of the interface between the aqueous water-ethanol region (left side) and the anethole oil-rich region (right side). Initially, the concentration profile of each species is a step function (first row of Fig. 4.7ABC), which is consistent with the initial condition $(4.7 \mathrm{hb})$ of the model. Different initial ethanol content in the drop accounts for the difference of the step heights in the figures.

In the close-up Figure 4.7D, once the diffusion starts, the discontinuity in the profile at $t=0 \mathrm{~s}$ is immediately smoothed with a new turning point emerging. The dynamics of the concentration profiles is apparent from the temporal curves at $t=10 \mathrm{~ms}, t=1 \mathrm{~s}, t=2 \mathrm{~s}$ and so on.

The concentration gradient at the interface induces a diffusive flux for each constituent towards or from infinity (anethole in Fig. 4.7D, ethanol and wa- 
ter in Fig. 4.7E). The step height at infinity remains unchanged due to the Dirichlet boundary condition $(4.7 \mathrm{kd})$. Thanks to the introduction of mathematical aspect of the Stefan problem, the interface has the freedom to shift towards the aqueous region (left side), for all the constituents in the system. After $20 \mathrm{~s}$, the developed concentration profiles in different cases are displayed in the second row of Figure 4.7 $\mathrm{ABC}$. The height of the new turning point, corresponding to the interfacial concentration $m_{\alpha s}^{\mathrm{d}}$ and $m_{\alpha s}^{\mathrm{h}}$ (ethanol, $\alpha=\mathrm{e}$, in Fig. 4.7 E), is fixed during the movement of the interface, which reflects the assumption of the stability of liquid-liquid equilibrium at interface.

\subsubsection{Diffusion paths theory and calculated results}

The diffusion path method was proposed by Kirkaldy and Brown [138, who mapped the composition of the ternary solution on its phase diagram. The values of the composition along the domains are mapped in the diagram as a line, known as diffusion path or composition path. It provides an effective way of representing the relationship between kinetic and thermodynamic aspects for a multiphase and multicomponent system.

In the spirit of the pioneering work by Ruschak and Miller [130, we predict the diffusion-induced spontaneous emulsification by examining the geometrical relationship between diffusion paths and binodal curve. If the diffusion path crosses the binodal curve between start and end point, supersaturation in the media is present, which induces spontaneous emulsification. It is noteworthy that the intersections of all considered diffusion paths with the binodal curve are far away from the plait point and the intersections are located at the edge of the ouzo effect region.

In ternary system, the path is determined by any two independent constituents. Since we know the evolution of the distribution of all species, we can mathematically express their relationship. We know that the coefficients $4.13 \mathrm{~b}$ ) are constant for a certain case, which implies that equation (4.9 a) is well-determined. Therefore, equation $(4.9 \mathrm{a})$ gives functional relationships $m_{\mathrm{w}}^{\mathrm{d}}=f_{1}\left(\frac{x}{\sqrt{t}}\right)$ and $m_{\mathrm{o}}^{\mathrm{d}}=f_{2}\left(\frac{x}{\sqrt{t}}\right)$. Upon substituting them into equation $\left.4.6 \mathrm{a}\right)$, we obtain the mathematical expression of the diffusion path in drop region,

$$
m_{\mathrm{e}}^{\mathrm{d}}=1-\mathcal{F}\left(m_{\mathrm{o}}^{\mathrm{d}}\right)-m_{\mathrm{o}}^{\mathrm{d}},
$$

where $\mathcal{F}=f_{1} \cdot f_{2}^{-1}$. By the same way, we obtain the expression of the diffusion path in the host liquid medium,

$$
m_{\mathrm{e}}^{\mathrm{h}}=1-m_{\mathrm{w}}^{\mathrm{h}}-\mathcal{G}\left(m_{\mathrm{w}}^{\mathrm{h}}\right),
$$

where $\mathcal{G}=g_{2} \cdot g_{1}^{-1}$, given $m_{\mathrm{w}}^{\mathrm{h}}=g_{1}\left(\frac{x}{\sqrt{t}}\right)$ and $m_{\mathrm{o}}^{\mathrm{h}}=g_{2}\left(\frac{x}{\sqrt{t}}\right)$. Notably, the mathematical expressions reveal that the diffusion path is independent of time and 

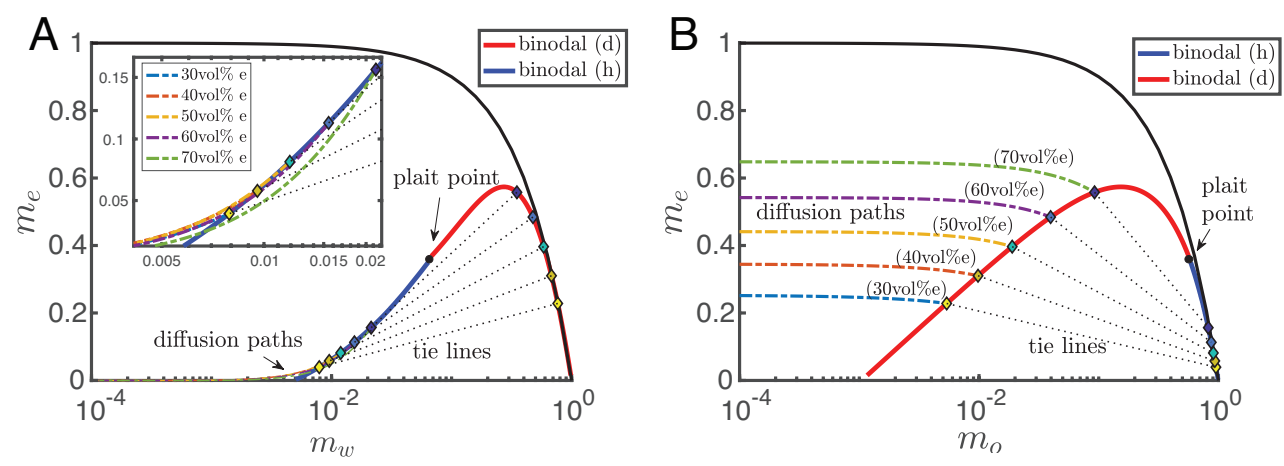

Figure 4.8: Phase diagrams showing the calculated diffusion paths in the water-ethanol phase diagram (A) and in the anethole-ethanol phase diagram (B). The oil-rich part of binodal curve is labeled in red and the water-rich part in blue. The black dotted lines are the tie lines. (A) The close-up figure (inserted) shows that the diffusion path in the host liquid passes through the binodal for the cases with high ethanol content ( $\geq 50 \mathrm{vol} \%$ ). (B) In contrast, in the aqueous phase, in all cases, there is no diffusion path crossing the binodal curve.

space. In other words, the set of the compositions at a certain position for different moments and the set for a certain moment among different positions share the same diffusion path. The composition at the end point of diffusion path lies on the binodal curve and corresponds to the interfacial composition, whereas the composition at the start point of the diffusion path indicates the composition at infinity, which is the same as the initial composition in the domain.

Figure 4.8 shows the calculated results, displayed in mass fraction phase diagrams of two independent constituents. Figure 4.8A shows the water-ethanol phase diagram, enhancing the oil-rich regime with w-in-o emulsions, whereas Figure $4.8 \mathrm{~B}$ shows the anethole-ethanol phase diagram, highlighting the waterrich regime with o-in-w emulsions. The binodal curve is divided by the plait point into two different colours, blue for oil-rich phase and red for water-rich phase. The tie lines, connecting the composition points belonging to both sides of the liquid-liquid equilibrium, were calculated by the method described in Section 4.5.2. Dot-dashed lines with different colours are the diffusion paths for different cases (same colour labeling for Fig. 4.8 $\mathrm{AB}$ ). The inserted closeup image in Figure 4.8 A reveals that, when the ethanol volume fraction in the aqueous phase is higher than 51.98 vol\%, corresponding to a transition mass fraction $m_{\mathrm{e} *}^{\mathrm{d}}$ calculated by the model, the diffusion path has to pass through the binodal curve to meet the equilibrium points (diamond dots) at the binodal. The segment of diffusion path that is below the binodal is in supersaturation condition, which indicates the appearance of w-in-o emulsions in oil-rich side (host liquid region). Whereas, in the water-rich side (Fig. 4.8 B), 

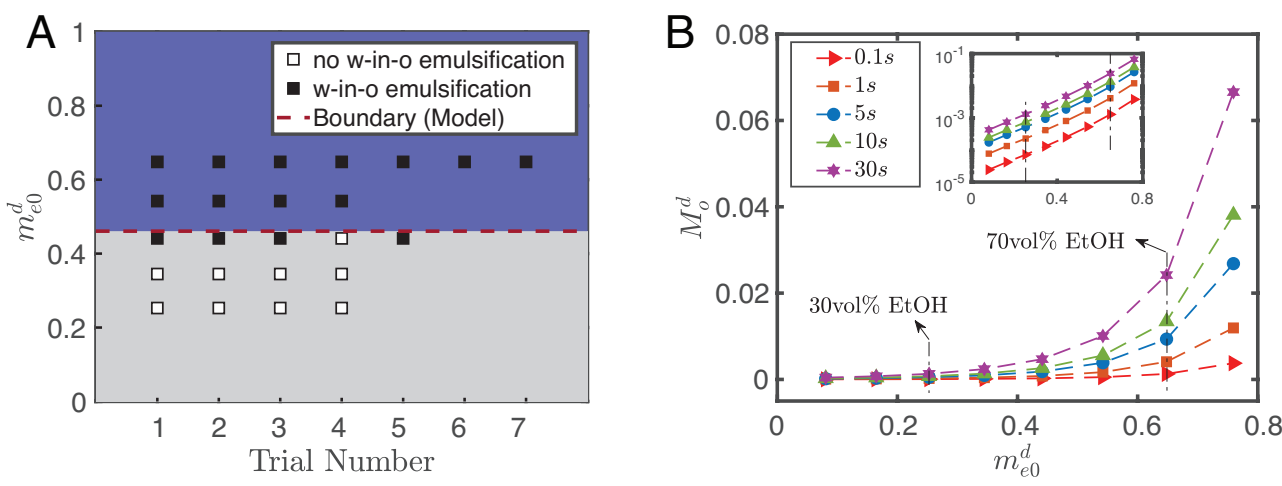

Figure 4.9: (A) Comparison between the model prediction and the experimental observation for the appearance of w-in-o emulsions outside the drop. The ordinate is the initial mass fraction of ethanol in the drop and the abscissa gives the trial number. The black and white dots are experimental observation data from Table 4.1 and denote presence and absence of emulsification, respectively. The red dashed line is a transition value calculated by the model, above which w-in-o emulsification is predicted to happen. (B) Calculation results of the total amount of oil transported into the drop as function of the initial ethanol content of the drop using the one-dimensional diffusion model. Different symbols present the entered oil amount at different moments. The inserted figure gives the same data on a log-log plot.

all the diffusion paths meet equilibrium points without intersecting the binodal curve. Therefore, according to the model, there is no supersaturation region induced by pure diffusion processes, which predicts the absence of o-in$\mathrm{w}$ emulsions in drop region. The time independence implies self-emulsification immediately occurs once diffusion process starts.

\subsubsection{Self-emulsification: comparison between model predictions and experimental observations}

For w-in-o emulsion, the comparison between model prediction and experimental observation is presented in Figure 4.9A. The vertical ordinate denotes the initial ethanol mass fraction in the water/ethanol drop and the abscissa gives the trial number, i.e. the individual experiments at this particular initial composition. The data points are from Table 4.1 black symbols indicate the presence of w-in-o emulsion; white symbol means that the w-in-o emulsion is absent. The red dashed line is the calculated transition percentage $m_{\mathrm{e} *}^{\mathrm{d}}$, above which (blue region) the model predicts the presence of w-in-o emulsion in the host liquid, otherwise absence (grey region). The comparison reveals the good prediction of the model. In the blue region, all data points are black, whereas in the grey region far from transition line, all the data dots are white. Around the transition line, both black and white data points exist.

For the o-in-w emulsion in the drop medium, no diffusion-tiggered self- 
emulsification was observed in experiments within half a minute (Tab. 4.1), which is consistent with the model prediction. However, oil microdroplets must appear since the ethanol content in the drop is reducing over time, while the oil content is increasing. At some point, the composition in the drop region will be supersaturated and cause the ouzo effect inside the drop. However, due to the infinite domain in the model with Dirichlet boundary conditions, the model cannot account for the long time behavior. The onset time for the oil microdroplets is at least more than half a minute, and has a negative correlation with initial content of ethanol in the drop as recorded in Table4.1.

Although we are unable to obtain the overall composition of the threedimensional drop from the one-dimensional model, it is possible to gain more understanding about the transport of anethole oil during the early stage by calculating the total amount of anethole transported into drop $M_{\mathrm{o}}^{\mathrm{d}}$. Its definition is given by integrating mass fraction of anethole $m_{\mathrm{o}}^{\mathrm{d}}$ on the interval of aqueous region $(-\infty, s)$

$$
M_{\mathrm{o}}^{\mathrm{d}}=\int_{-\infty}^{s}\left(m_{\mathrm{o}}^{\mathrm{d}}-m_{\mathrm{o} 0}^{\mathrm{d}}\right) \mathrm{d} x .
$$

Then we have

$$
M_{\mathrm{o}}^{\mathrm{d}}=\left(m_{\mathrm{o} s}^{\mathrm{d}}-m_{\mathrm{o} 0}^{\mathrm{d}}\right) s+\frac{\left(s m_{\mathrm{o} s}^{\mathrm{d}}-m_{\mathrm{o} 0}^{\mathrm{d}}\right) s}{\sqrt{\pi} \mathcal{R}_{\mathrm{o}} \mathcal{K}_{\mathrm{o}}} \frac{e^{-\mathcal{R}_{\mathrm{o}}^{2} \mathcal{K}_{\mathrm{o}}^{2}}}{\operatorname{erf}\left(\mathcal{R}_{\mathrm{o}} \mathcal{K}_{\mathrm{o}}\right)+1} .
$$

Figure $4.9 \mathrm{~B}$ shows that a higher ethanol percentage in drop $m_{\mathrm{e} 0}^{\mathrm{d}}$ leads to a higher rate of transport of oil into the drop $M_{\mathrm{o}}^{\mathrm{d}}$. The inserted figure reveals that it is an exponential growth relationship. So the difference in the oil amount in the drop caused by the ethanol content increases over time (from red solid $\triangleright$ dot line, at $0.1 \mathrm{~s}$, to purple solid $\$$ dot line, at $30 \mathrm{~s}$ ). This gives a possible explanation for the experimental observation, as a higher ethanol concentration in the drop leads to more oil after the early stage of the diffusion process, which favours the occurrence of the ouzo effect inside the drop.

\subsubsection{Model evaluations}

Although the model provides information on the mass transport of the multiphase and multicomponent diffusion process, as well as a good prediction to the behaviour of spontaneous emulsification, it is subject to the following limitation: (i) The diffusion model is developed as a one-dimensional model without consideration of flow motion, i.e. a pure multiphase and multicomponent diffusion process; (ii) The dependence of diffusivity on the local species concentration is disregarded and off-diagonal diffusion terms, i.e. as in the Maxwell-Stefan theory, are not considered; (iii) We apply Dirichlet boundary 
conditions at infinity, which implies that the model is only applicable in the early stage of the diffusion process. In the long-time limit, the finite size of the regions, in particular of the drop, will become important, since there is not an infinite reservoir for the species. (iv) The detailed process of phase separation at interface is not considered, as we apply an instantaneous liquid-liquid equilibrium assumption;

The flow motion in system has a big impact on the position where emulsification takes place. A strong flow rate has the capacity to prevent emulsification by changing the local concentrations or by directly dissolving the nucleated emulsion. Therefore, it is necessary to have an investigation on the fluid dynamics of the system in Section 4.7

\subsection{Discussion on the fluid dynamics of the system}

\subsubsection{Scaling analysis}

In the studied system, diffusion-induced advection, in the laminar flow regime, can in turn play a significant role for the diffusion process, i.e. diffusion and advection are highly coupled in the system. The diffusion causes non-uniformities in the species distribution in both drop and host liquid media, which drives bulk flow motions (advection). The flow reorganises the concentration field and consequently, in turn, affects the diffusion. The generated advection mainly encompasses solutal Marangoni flow and buoyancy-driven flow, i.e. solutal natural convection, whereas thermal effects are negligible compared to the two of them. As solutal Marangoni flow and buoyancy-driven flow have alternating dominance inside respective outside the drop their impacts on diffusion can be treated separately. To confirm that only one of the two respective flows is dominant in the respective domain, we perform the following scaling analysis.

The characteristic velocity of the solutal Marangoni flow $U_{\mathrm{M}}$ scales like

$$
U_{\mathrm{M}} \sim \Delta \gamma / \mu,
$$

where $\mu$ is the dynamic viscosity, and $\Delta \gamma$ is the interfacial surface tension difference caused by compositional variations. The characteristic velocity associated with the buoyancy-driven convection $U_{\mathrm{B}}$ is obtained by balancing the viscous dissipation rate within the convection roll with the gaining rate of potential energy due to gravity [147], i.e.

$$
\int \mu(\nabla u)^{2} \mathrm{~d} V \sim \Delta \rho g U_{\mathrm{B}} \ell^{3},
$$


where the left side scales as $\mu \ell\left(U_{\mathrm{B}}\right)^{2}$. Here $\ell$ is the characteristic length scale and $g$ is the gravitational constant. Hence, the characteristic velocity of the buoyancy-driven flow $U_{\mathrm{B}}$ scales as

$$
U_{\mathrm{B}} \sim \Delta \rho g \ell^{2} / \mu .
$$

With equations 4.22 and 4.21 , we can estimate the ratio of buoyancy-driven flow to Marangoni flow by

$$
\frac{U_{\mathrm{B}}}{U_{\mathrm{M}}} \sim \frac{\Delta \rho g \ell^{2}}{\Delta \gamma} .
$$

We can note the ratio is a Bond number, which measures the importance of gravitational forces compared to interfacial surface tension. The Bond number is proportional to $\ell^{2}$, which indicates that relative importance of the two flow mechanisms has a strong dependence on the spatial scale of the domain.

We can estimate the ratio inside the drop by taking the drop radius $R \sim$ $0.75 \mathrm{~mm}$ as the length scale $\ell$. Since the interfacial surface tension is compositiondependent and varies during drop dissolution, we estimated the difference $\Delta \gamma \sim 12.1 \mathrm{mNm}^{-1}$, as half of the interfacial surface tension between pure water and anethole oil [136]. The density difference is selected as the biggest density difference between water and ethanol, i.e. $\Delta \rho=\rho_{\mathrm{w}}-\rho_{\mathrm{e}}\left(\sim 211 \mathrm{~kg} / \mathrm{m}^{3}\right)$. The estimation shows that $U_{\mathrm{B}} \sim U_{\mathrm{M}} / 10$, which implies Marangoni flow inside the drop is prevailing. Outside the drop, the length scale $\ell$ is selected as the host liquid depth, $\ell=7.5 \mathrm{~mm}$. Then the estimate of the velocity ratio becomes $U_{\mathrm{B}} \sim 10 U_{\mathrm{M}}$, which indicates that buoyancy-driven flow is dominating outside the drop.

\subsubsection{Flow motions and its influence on spontaneous emulsification}

Taking the understanding of the flow motion in the system from the scaling analysis, we disregard the Marangoni flow in the following discussion about the hydrodynamics in the host liquid (large scale domain), whereas the buoyancy effect is neglected inside the drop (small scale domain). The hydrodynamics outside and inside drop are discussed successively.

As water-ethanol drop dissolves, ethanol diffuses into the surrounding anethole oil. Then, buoyancy starts to play a role, as ethanol is less dense than water and anethole oil, i.e. $\rho_{\mathrm{e}}<\rho_{\mathrm{o}}$ and $\rho_{\mathrm{e}}<\rho_{\mathrm{w}}\left(\rho_{\mathrm{w}}=998 \mathrm{~kg} / \mathrm{m}^{3}, \rho_{\mathrm{e}}=787 \mathrm{~kg} / \mathrm{m}^{3}\right.$, and $\rho_{\mathrm{o}}=988 \mathrm{~kg} / \mathrm{m}^{3}$ at $22^{\circ} \mathrm{C}$ ). The surrounding ethanol-rich oil floats up in a form of the solute plume, causing an upwelling flow. Simultaneously, fresh oil liquid far from the drop replenishes to achieve mass continuity, as sketched in Figure 4.1A. So a convection flow outside the drop forms as a consequence, which is clearly observed through PIV measurements (Fig. 4.1B). In the region next to the tropic of capricorn of the drop and not far from the drop 
surface, there is weak flow, enclosed by the yellow circle in the close-up image. It indicates a subtle influence of the convection on the diffusion process in this region, whereas in other regions, the refresh oil brought by the relatively strong flow prevents the formation of a local supersaturation. This is the reason that accounts for the appearance of w-in-o microdroplets in the certain position with the weak flow rate.

The generation of the buoyancy-driven convection affects the distribution of the diffusion rate along the drop surface. The convection flow brushes away the diffused ethanol next to drop surface and varies the concentration distribution of ethanol in the surrounding oil. Around the equator of the drop the concentration boundary layer is thin, due to the intense inflow of bulk oil without ethanol (Fig. 4.1B): the normal ethanol concentration gradient outside the liquid-liquid interface $\left(\partial_{r} c^{\mathrm{e}}\right)_{\text {side }}$ has a steep slope. At the top of the drop, the ethanol concentration gradient $\left(\partial_{r} c^{\mathrm{e}}\right)_{\text {top }}$ may also be increased, but only to a lower extent, as the replenishing oil is already contaminated with ethanol (and water) during its travel along the drop surface. Due to the boundary condition, the replenishment of fresh oil caused by the convection is suppressed near the corner of the drop, where the ethanol concentration gradient $\left(\partial_{r} c^{\mathrm{e}}\right)_{\text {C.L. }}$ is much less effected. Therefore, the buoyancy-driven convection predominantly enhances the diffusion flux near the drop equator rather than that above the drop or in the contact region and, as a consequence, the radial gradients of ethanol concentration along the drop surface obey the a relationships

$$
\begin{aligned}
& \left(\partial_{r} c^{\mathrm{e}}\right)_{\text {side }}>\left(\partial_{r} c^{\mathrm{e}}\right)_{\text {top }}, \\
& \left(\partial_{r} c^{\mathrm{e}}\right)_{\text {side }}>\left(\partial_{r} c^{\mathrm{e}}\right)_{\text {C.L. }} .
\end{aligned}
$$

The two different gradients of the ethanol concentration result in a inhomogeneneous ethanol concentration along the inside of the interface. The intense concentration gradient of ethanol around the equator $\left(\partial_{r} c^{\mathrm{e}}\right)_{\text {side }}$ generates a large ethanol diffusion flux, resulting in a locally higher water concentration inside the drop. As the interfacial surface tension between oil phase and aqueous phase has a positive correlation with the water concentration in aqueous phase, the interfacial surface tension has gradients from the south pole and north pole towards the equator. Thus, two solute Marangoni convections arise following the gradients, as sketched in Figure 4.1A. Meanwhile, the high water concentration around the equator of the drop accounts for the prefered emulsification there (Fig. 4.2 C). 


\begin{tabular}{c|l|c}
\hline Symbol & Description & unit \\
\hline index $\alpha$ & species: water (w); ethanol (e); anethole oil (o) & \\
superscript d & drop region & \\
superscript h & host liquid region & $\mathrm{m}$ \\
$s$ & position of drop\&host-liquid interface & \\
$m_{\alpha}^{\mathrm{d}}$ & mass fraction (drop region) & \\
$m_{\alpha}^{\mathrm{h}}$ & mass fraction (host liquid region) & \\
$m_{\alpha 0}^{\mathrm{d}}$ & initial mass fraction (drop region) & \\
$m_{\alpha 0}^{\mathrm{h}}$ & initial mass fraction (host liquid region) & \\
$m_{\alpha s}^{\mathrm{d}}$ & mass fraction at interface (drop region) & \\
$m_{\alpha s}^{\mathrm{h}}$ & mass fraction at interface (host liquid region) & \\
$M_{\alpha s}^{\mathrm{d}}$ & $M_{\mathrm{a}}^{\mathrm{d}}=\int_{-\infty}^{s} m_{\mathrm{a}}^{\mathrm{d}}-m_{\mathrm{a} 0}^{\mathrm{d}} \mathrm{d} x$ & $\mathrm{~m}$ \\
$D_{\alpha}^{\mathrm{d}}$ & diffusivity (drop region) & $\mathrm{m}^{2} / \mathrm{s}$ \\
$D_{\alpha}^{\mathrm{h}}$ & diffusivity (host liquid region) & $\mathrm{m}^{2} / \mathrm{s}$ \\
$\mathcal{B}_{\alpha}^{1}, \ldots, \mathcal{B}_{\alpha}^{4}$ & undetermined coefficients & \\
$\lambda$ & prefactor defined as $s / \sqrt{t}$ & $\mathrm{~m} / \mathrm{s}^{\frac{1}{2}}$ \\
$D_{s}$ & $\lambda^{2}$ & $\mathrm{~m}^{2} / \mathrm{s}$ \\
$\mathcal{K}_{\alpha}$ & $\lambda / \sqrt{D_{\alpha}^{\mathrm{h}}}$ & \\
$\mathcal{R}_{\alpha}$ & $\sqrt{D_{\alpha}^{\mathrm{h}} / D_{\alpha}^{\mathrm{d}}}$ & \\
$\chi_{\mathrm{o}}^{\mathrm{w}}$ & $\sqrt{D_{\mathrm{o}}^{\mathrm{h}} / D_{\mathrm{w}}^{\mathrm{h}}}$ & \\
$x_{\alpha}$ & mole fraction of species $\alpha$ & \\
$\gamma_{\alpha}$ & activity coefficient of species $\alpha$ & \\
\hline
\end{tabular}

Table 4.2: All quantities used in the model for the ternary liquid.

\subsection{Summary and conclusions}

We have experimentally presented the rich phenomena of water/ethanol drops dissolving in oil as host liquid, which encompass w-in-o emulsification outside the drop, o-in-w emulsification inside the drop, buoyancy-driven convection dominating outside the drop, and prevailing solutal Marangoni convection inside the drop. O-in-w emulsification occurs around half minute later than w-in-o emulsification and w-in-o emulsification does not occur when reducing the initial ethanol concentration of the drop.

A quantitive understanding and the predictions of the diffusion-induced emulsification were theoretically achieved by developing a one-dimensional multiphase and multicomponent diffusion model, which incorporates thermodynamical equilibrium theory and diffusion path theory. The prediction of the model agrees with experimental observations: diffusion-triggered w-in-o emul- 
sification occurs when the drops have a ethanol content higher than 51.98 vol\%; o-in-w emulsification cannot be induced by pure diffusion. Due to the infinite domain and the Dirichlet boundary conditions used in the model, the model is only applicable for the early stage of the multiphase diffusion process. In practice, the continuous reduction of ethanol and increasing of oil in the drop lead to the occurrence of the o-in-w emulsification in a long time, which is thereby independent of the initial ethanol content of the drop.

A scale analysis and the experimental investigation of the diffusion-induced flow motion were performed to gain insight into its influence on the emulsification process. By the scale analysis, we demonstrated that in drop domain, the solute Marangoni flow prevails over the buoyancy-driven flow, while in host liquid domain, the latter dominates. The buoyancy-driven convection enhances the ethanol diffusion rate around the equator of the drop, which gives rise to a reduction of the local ethanol concentration around the equator of the drop, arising of two Marangoni convection rolls inside the drop with opposite directions, and the generation of prioritized position for o-in-w emulsification. Due to the buoyancy-driven flow, fresh oil is replenished at the interface. Therefore w-in-o emulsification can only occur around a region next to the tropic of capricorn of the drop, where only weak flow and replenishment is present.

Although in this paper we provided a systematic study about the emulsification triggered by the dissolution of multicomponent drop in a host liquid, further investigations and discussions are expected. A more comprehensive model can be generated, either by considering the finite domain size and the appropriate boundary conditions, or by taking fluid motion into account and developing an axisymmetric model. Experimentally, the influence of temperature, different chemical systems, or different geometries by varying the contact angle of the drop are appealing open questions to explore. A better understanding of the dissolution of multiphase and multicomponent systems may provide valuable information for the investigation of multiphase systems and spontaneous emulsifications in general. We also hope our work may provide contribution to industrial application, such as modern liquid-liquid microextraction techniques. 


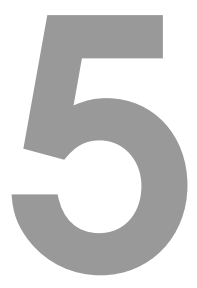

\section{Evaporating pure, binary \& ternary sessile drops f}

The Greek aperitif Ouzo is not only famous for its specific anise-flavored taste, but also for its ability to turn from a transparent miscible liquid to a milkywhite colored emulsion when water is added. Recently, it has been shown that this so-called Ouzo effect, i.e. the spontaneous emulsification of oil microdroplets, can also be triggered by the preferential evaporation of ethanol in an evaporating sessile Ouzo drop, leading to an amazingly rich drying process with multiple phase transitions [H. Tan et al., Proc. Natl. Acad. Sci. USA 113(31) (2016) 8642]. Due to the enhanced evaporation near the contact line, the nucleation of oil droplets starts at the rim which results in an oil ring encircling the drop. Furthermore, the oil droplets are advected through the Ouzo drop by a fast solutal Marangoni flow. In this article, we investigate the evaporation of mixture droplets in more detail, by successively increasing the mixture complexity from pure water over a binary water-ethanol mixture to the ternary Ouzo mixture (water, ethanol and anise oil). In particular, axisymmetric and full three-dimensional finite element method simulations have been performed on these droplets to discuss thermal effects and the complicated flow in the droplet driven by an interplay of preferential evaporation, evaporative cooling and so-

${ }^{*}$ Based on: C. Diddens, H. Tan, P. Lv, M. Versluis, J.G.M. Kuerten, X. Zhang, and D. Lohse, Evaporating pure, binary and ternary droplets: thermal effects and axial symmetry breaking, Journal of fluid mechanics 823, 470-497 (2017).

${ }^{\dagger}$ Numerical work performed by C. Diddens. 
lutal and thermal Marangoni flow. By using image analysis techniques and micro-PIV measurements, we are able to compare the numerically predicted volume evolutions and velocity fields with experimental data.

\subsection{Introduction}

Evaporation of sessile droplets is a ubiquitous phenomenon which is utilized in a wide-spread range of applications, e.g. inkjet printing, coating and spray cooling. The pioneering work of Deegan et al. 38, explaining the so-called coffee-stain effect, incented the scientific investigation of evaporating droplets in general.

While the evaporation process of droplets consisting of a pure liquid is mainly understood [35], multi-component droplets show in general far more complex evolutions during evaporation. This is due to the complicated coupling of multi-component evaporation, flow of the mixture and possibly also thermal effects. Even for binary mixtures, the presence of the second component can result in non-monotonic contact angle evolutions [51, 148 151, initial condensation of the less volatile component [152] or entrapped residuals of the more volatile component at later times [56, 57, 110, 111]. Furthermore, the flow in the droplet is primarily driven by the solutal Marangoni effect. In particular, it has been shown that water-ethanol droplets exhibit initially chaotic and highly non-axisymmetric flows, followed by a fast transition to nearly axisymmetric flow and outward radial flow towards the end of the lifetime [59, 100, 153]. By adding surfactants and surface-absorbed polymers, the Marangoni flow can be controlled, leading to homogeneous deposition patterns [72]. Neighboring binary mixture droplets can also interact through the vapor phase which allows for the assembly of intriguing autonomous fluidic machines [154. Also the dissolution of a binary droplet in a third liquid shows highly nontrivial and unexpected behavior [17, 127.

Recently, it has been shown that ternary mixture droplets, as the next more general liquid, can show an even richer evolution process: By investigating the evaporation of an Ouzo drop (ethanol, water and anise oil), we have revealed multiple phase transitions, where the drop temporarily changes its appearance from an initially transparent liquid to a milky-white colored emulsion [101]. The reason lies in the so-called Ouzo effect [41, i.e. the spontaneous emulsification of oil microdroplets once the local ethanol concentration has reduced by preferential evaporation below a specific threshold. Since the evaporation rate for droplets with contact angles below $90^{\circ}$ is highest at the contact line, the onset of the Ouzo effect starts near the rim which additionally results in an oil ring encircling the drop. While most of the oil droplets are quickly trans- 
ported by solutal Marangoni flow through the entire droplet, also sessile oil droplets immersed in the surrounding drop were observed - so-called surface nanodroplets [36, 37, 63]. If the contact angle of an Ouzo droplet exceeds $90^{\circ}$, the Ouzo effect initially occurs close to the apex, i.e. where the evaporation is pronounced in this case, and no persistent oil ring formation can be observed [136]. Instead, a continuous oil phase wraps up the drop due to Marangoni forces.

Although the numerical results for the volume evolution of an Ouzo droplet are in good agreement with the experimental data, there are some drawbacks in the study of Tan et al. [101]: On the one hand, the applied lubrication theory is only valid for very flat droplets, but the experimentally found contact angle of the Ouzo droplet temporarily exceeds $80^{\circ}$, which causes a considerable inaccuracy of the predicted flow velocity [110, 111]. On the other hand, it is well known from the afore-mentioned experiments that the flow in ethanolwater droplets is initially highly non-axisymmetric, which cannot be covered by the used axisymmetric model. Finally, the influence of the latent heat of evaporation has been neglected and the relative humidity had to be adjusted in order to match the experimental volume evolution. In particular on a thin substrate, as used in the experiment, thermal effects can play a crucial role 111, 136.

In this study, we take advantage of a finite element method model to investigate the flow velocity inside evaporating multi-component droplets in more detail, i.e. without being subject to the mentioned limitations of our previous study. The model comprises multicomponent evaporation, evaporative cooling, thermal and solutal Marangoni flow as well as compositionand temperature-dependent fluid properties. We start our investigation with a pure water droplet as the simplest case and successively generalize it to a binary water-ethanol droplet and to the ternary Ouzo droplet. This procedure allows us to discuss possible disagreements between simulations and experiments for complicated mixtures in the light of the agreement for simpler mixtures. In the next step, the previously axisymmetric model is generalized to a full three-dimensional variant. Thereby, we are able to investigate the aforementioned axial symmetry breaking of the flow and compare it to micro-particle-image-velocimetry (micro-PIV) measurements and to undertake a qualitative analysis by confocal microscopy.

Details about the performed experiments are described in section 5.2. In section 5.3 , the axisymmetric finite element method model is outlined and its results are compared with the corresponding experimental data. The model is generalized to three dimensions in section 5.4 to account for non-axisymmetric flow and the numerical results are compared with experimental micro-PIV 
measurements.

\subsection{Experimental setup}

\subsubsection{Droplet composition}

In total, we have experimentally investigated the evaporation of three different types of droplets, namely a pure water droplet, a binary water-ethanol droplet and a ternary Ouzo droplet. The liquids used for these droplets were pure Milli-Q water [produced by a Reference A+ system (Merck Millipore) at $18.2 \mathrm{M} \Omega \cdot \mathrm{cm}\left(\right.$ at $25^{\circ} \mathrm{C}$ )], a mixture of $37.88 \%$ (wt/wt) Milli-Q water and $62.12 \%$ (wt/wt) ethanol [Sigma-Aldrich; $\geq 99.8 \%$ ], and a mixture of $37.24 \%$ (wt/wt) Milli-Q water, $61.06 \%$ (wt/wt) ethanol and $1.70 \%$ anise oil [SigmaAldrich], respectively. Furthermore, for the experimental micro-PIV measurements described later on in section 5.2.3, tracer particles have been added to the binary water-ethanol droplet.

\subsubsection{Setup and image analysis}

The droplets were deposited through a teflonized needle [Hamilton; 8646-02] by a motorized syringe pump [Harvard; PHD 2000] on a flat hydrophobic octadecyltrichlorosilane (OTS)-glass surface with a thickness of $d_{\mathrm{s}}=0.17 \mathrm{~mm}$. The advancing and receding contact angles of water on the surface are $112^{\circ}$ and $98^{\circ}$, respectively. The entire evaporation process of the droplets was recorded by a CCD camera [Ximea; MD061MU-SY, 3 frames per second (fps) at $1372 \times 1100$ pixel resolution] equipped with a long-distance microscope system [Infinity; Model K2 DistaMax] for side-view recordings and a digital SLR camera [Nikon D750] equipped with a CMOS sensor [24 frames per seconds (fps) at $1920 \times 1080$ pixels resolution] attached to a high-magnification zoom lens system [Thorlabs; MVL12X3Z] for top-view recordings. The side-view illumination was provided by a homemade collimated LED source. A universal hand-held probe [Omega; HH-USD-RP1, accuracy relative humidity is $\pm 2 \%$ over 10 to $90 \% @ 25{ }^{\circ} \mathrm{C}$ and a temperature accuracy of $\left.\pm 0.3 \mathrm{~K} @ 25{ }^{\circ} \mathrm{C}\right]$ was used to measure the relative humidity and temperature in the laboratory at a sampling rate of one per second. A similar sketch of the setup is described in detail in Tan et al. [101].

The image analysis was performed by a custom-made MATLAB program, through which all of the geometric parameters at every frame were successfully determined. For the millimetric droplets in this study, the characteristic lengths of the droplets are smaller than the capillary length scale, which is equal to $2.7 \mathrm{~mm}$ for water and $1.7 \mathrm{~mm}$ for ethanol. Thus, gravity effects influ- 


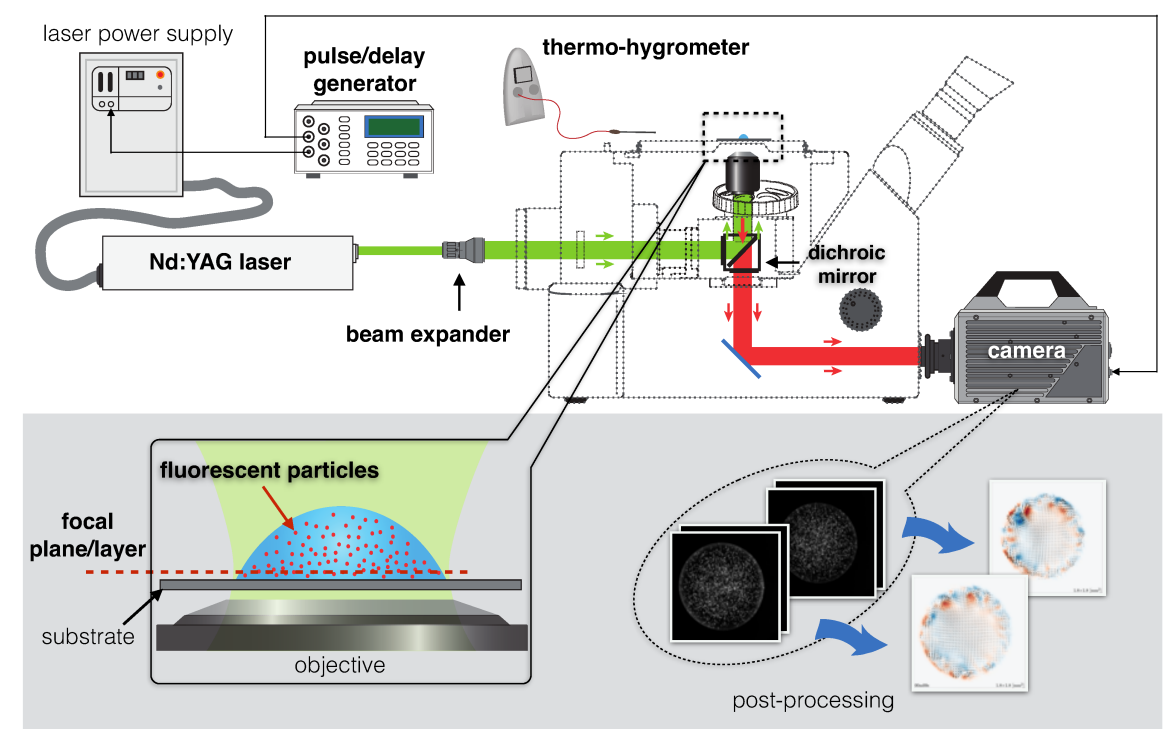

Figure 5.1: Experimental setup of the micro-PIV system. The dual-cavity laser and the highspeed camera are synchronized to record consecutive image pairs. To apply fluorescence techniques, the inverted microscope is equipped with a dichroic cube and the droplet is seeded with fluorescent particles. The focal plane of the microscope is placed at the bottom of the droplet, i.e. close and parallel to the substrate.

encing the shape of the droplets can be disregarded. For pure water droplets and binary water-ethanol droplets, a spherical-cap shape assumption was used over the whole evaporation process. The program fits a circle to the contour of the droplet silhouette in side view. The contact angle $\theta$ was calculated based on the position of the intersection of the base line and the fitted circles. Since Ouzo droplets temporarily show a very characteristic deviation from the spherical cap shape due to the appearance of an oil ring at the rim, only the top part of the contour above the oil ring was fitted by a circle. A polynomial fit was applied to the profile of the oil ring and gave the contact angle $\theta$. The contact angle $\theta^{*}$ of the spherical cap shaped top part was calculated based on the position of the intersection point where the fitted line of the oil ring profile and the fitted circle of the top contour cross. The droplet volume $V$ was calculated by integrating the volumes of horizontal disk layers and assuming rotational symmetry of each layer with respect to the vertical axis.

\subsubsection{Micro particle image velocimetry (micro-PIV)}

To determine the velocity in the binary water-ethanol droplet, Micro Particle Image Velocimetry (micro-PIV) measurements were performed. To that end, the binary water-ethanol mixture was seeded with fluorescent particles. Per 
$1 \mathrm{~mL}$ of the water-ethanol solution, $30 \mu \mathrm{L}$ of aqueous tracer particle solution [microParticles GmbH; PS-FluoRed-5.0: Ex/Em 530nm/607nm] was used. The diameter of these tracer particles is $d_{\mathrm{p}}=5 \mu \mathrm{m}$.

An overview of the experimental micro-PIV setup is given in Figure 5.1. It consists of a dual-cavity Nd:YAG laser [Litron; NANO S 65-15PIV], a highspeed camera [Photron; Fastcam SA-X2 $64 \mathrm{~GB}$ ] and an inverted microscope [Olympus; GX-51] which is equipped with a dichroic cube. The laser and the high-speed camera are synchronized by a pulse/delay generator [BNC; Model-575]. The laser beam illuminates the droplet from below through a $10 \times$ magnification objective lens with a numerical aperture (NA) of 0.30 (zoom-in of Figure 5.1). The objective focal plane was placed just above the surface at approximately $13 \mu \mathrm{m}$ to match the thickness of the focal plane, or the depth of field (DOF), which was calculated to be $13.4 \mu \mathrm{m}$. Thus, we had a sharp image of the tracer particles closest to the substrate.

In this way, 10 consecutive image pairs with an interframing time of $4 \mathrm{~ms}$ were taken per second. To calculate the velocity field, the obtained images were first post-processed with a custom MATLAB code to enhance the contrast. Then, the image pairs were analyzed with PIVlab [132, 133, using an interrogation window of $64 \times 64$ pixels, corresponding to $128 \mu \mathrm{m} \times 128 \mu \mathrm{m}$. An interrogation window overlap of $75 \%$ leads to a $32 \mu \mathrm{m}$ vector spacing.

To qualify the degree to which the tracer particles exactly follow the flow, we analyzed the Stokes number and the ratio of Stokes number to a buoyancycorrected Froude number [134]. The Stokes number is defined as $S t \equiv t_{0} u_{\max } / r_{\mathrm{c}}$, where $t_{0} \equiv \frac{\rho_{\mathrm{p}} d_{\mathrm{p}}^{2}}{18 \mu_{\mathrm{f}}}\left(1+\rho_{\mathrm{f}} /\left(2 \rho_{\mathrm{p}}\right)\right)$ is the characteristic time, $u_{\max }$ the maximum fluid velocity $\left(\sim 10 \mathrm{mms}^{-1}\right), \rho_{\mathrm{p}}$ the density of the tracer particles $\left(1.04 \mathrm{~g} \mathrm{~cm}^{-3}\right)$, and $\mu_{\mathrm{f}}$ the dynamic viscosity of the droplet liquid $\left(\sim 10^{-3} \mathrm{Pas}\right)$. The factor $\left(1+\rho_{\mathrm{f}} /\left(2 \rho_{\mathrm{p}}\right)\right)$ accounts for the added mass force [135]. The buoyancy-corrected Froude number is defined as $F r \equiv \frac{u_{\max }^{2} / r_{\mathrm{c}}}{\left(1-\rho_{\mathrm{f}} / \rho_{\mathrm{p}}\right) g}$, where $\rho_{\mathrm{f}}$ is the density of the droplet liquid $\left(0.977 \mathrm{~g} \mathrm{~cm}^{-3}\right.$ for water and $0.789 \mathrm{~g} \mathrm{~cm}^{-3}$ for ethanol) and $g$ is the gravitational acceleration. The calculated values for $S t$ and $S t / F r$ are $\sim 10^{-5}$ and $\sim 10^{-2}$ respectively, which indicate that the tracer particles are truly tracers for the liquid flow inside the evaporating droplets.

The side-view recording setup (section 5.2.2) was coupled to the micro-PIV setup to monitor the geometric shape evolution of the evaporating droplet synchronized with the flow inside. 


\subsection{Axisymmetric investigation}

In a first step in the model used in the numerical simulations, the drop is assumed to be axisymmetric. Although the flow velocity investigation later on in section 5.4 shows that this symmetry is broken, the assumption of axisymmetry drastically reduces the computation time. As shown by Diddens et al. [110, the exact details of the flow in a droplet are not relevant for macroscopic quantities as e.g. the volume evolution $V(t)$, whenever the flow is driven by an intense Marangoni flow. This fact allows to discuss e.g. the influence of the latent heat of evaporation on the droplet evolution within an axisymmetric model.

\subsubsection{Axisymmetric finite element method}

In the following an outline of the axisymmetric finite element method is given. A detailed description of the model can be found in the work of Diddens et al. [111. As depicted in Figure 5.2, the space, represented by axisymmetric cylindrical coordinates $(r, z)$, is separated into individual subdomains, namely the gas phase $\Omega_{\mathrm{g}}$, the droplet liquid $\Omega_{\mathrm{l}}$, the substrate $\Omega_{\mathrm{s}}$ with a finite thickness $d_{\mathrm{s}}$ and the air below the substrate $\Omega_{\mathrm{b}}$. The droplet shape, defined by the liquidgas interface $\Gamma_{\mathrm{lg}}$, is assumed to be always in a spherical-cap shape. According to the ellipticity measurements of Tan et al. [101, this is a good approximation, at least as long as the oil ring in case of the Ouzo droplet is not taken into account.

The following derivation of the model considers the Ouzo droplet, but it can easily be simplified to the pure water droplet and the binary water-ethanol droplet. The composition-dependence of the physical fluid properties, i.e. viscosity $\mu$, diffusivity $D$, mass density $\rho$, surface tension $\sigma$ and thermodynamic activities, are locally considered by fitting experimental data for water-ethanol mixtures [67-69] and using thermodynamic models [70, 77]. Due to the small initial concentration of anise oil in the Ouzo mixture, its influence on the liquid properties is not considered. The temperature-dependence of the surface tension is also considered based on the experimental data of Vazquez et al. [68] to account for thermal Marangoni flow. Plots of all these relations can be found in the work of Tan et al. [101] and Diddens et al. [110].

In the gas phase, the diffusion equation for the vapor concentration $c_{\alpha}$ with $\alpha=\mathrm{e}$ and $\alpha=\mathrm{w}$ for ethanol and water, respectively, has to be solved. With the vapor diffusivity $D_{\alpha}^{\mathrm{g}}$ of $\alpha$ in air, this reads

$$
\partial_{t} c_{\alpha}=D_{\alpha}^{\mathrm{g}} \nabla^{2} c_{\alpha}
$$

The anise oil is assumed to be non-volatile due to the low vapor pressure of 

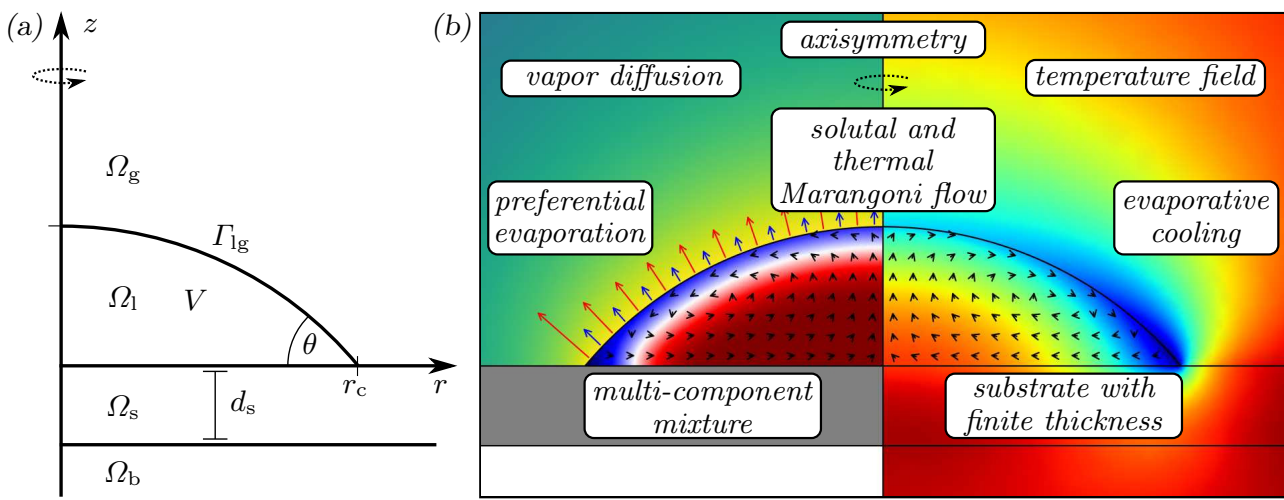

Figure 5.2: (a) The problem is expressed by axisymmetric cylindrical coordinates. The individual domains $\Omega_{\mathrm{g}}, \Omega_{\mathrm{l}}, \Omega_{\mathrm{s}}$ and $\Omega_{\mathrm{b}}$ are the gas phase, the liquid droplet, the substrate with finite thickness $d_{\mathrm{s}}$ and the air below the substrate, respectively. (b) The model solves the coupled processes of vapor-diffusion-limited mixture evaporation, multi-component flow with composition-dependent quantities and driven by solutal and thermal Marangoni flow, as well the temperature field.

trans-anethole. As boundary condition of (5.1) for $(r, z) \rightarrow \infty$, the ambient vapor concentrations $c_{\alpha, \infty}$ have to be imposed. While there is no ethanol vapor present far away from the droplet, i.e. $c_{\mathrm{e}, \infty}=0$, the water vapor concentration can be expressed by the ideal gas law:

$$
c_{\mathrm{w}, \infty}=\phi \frac{M_{\mathrm{w}} p_{\mathrm{w}, \mathrm{sat}}\left(T_{\infty}\right)}{R T_{\infty}} .
$$

Here, $\phi$ is the relative humidity, $R$ is the ideal gas constant, $T_{\infty}$ is the room temperature and $p_{\mathrm{w}, \mathrm{sat}}$ is the saturation pressure, which temperaturedependence is given by the Antoine equation. At the liquid-gas interface $\Gamma_{\lg }$, the vapor-liquid equilibrium concentration $c_{\alpha, \mathrm{VLE}}$ is imposed, which can be calculated via Raoult's law, i.e. by

$$
c_{\alpha, \mathrm{VLE}}=\gamma_{\alpha} x_{\alpha} \frac{M_{\alpha} p_{\alpha, \mathrm{sat}}(T)}{R T}
$$

The vapor-liquid equilibrium couples the vapor concentration at the droplet interface to the liquid mole fraction $x_{\alpha}$, where non-idealities are considered by the activity coefficient $\gamma_{\alpha}$. Since (5.3) has to be evaluated at the local temperature, the evaporation rates are also coupled to the temperature field $T$. From the solution of (5.1), one can directly calculate the diffusive vapor flux at the interface:

$$
\boldsymbol{J}_{\alpha}^{\mathrm{g}}=-D_{\alpha}^{\mathrm{g}} \nabla c_{\alpha} \cdot \boldsymbol{n}_{\lg } \cdot
$$


The mass transfer rates $j_{\mathrm{w}}^{\mathrm{lg}}$ and $j_{\mathrm{e}}^{\mathrm{lg}}$ can be determined from the diffusive vapor fluxes via the coupled mass transfer jump conditions

$$
j_{\alpha}^{\mathrm{lg}}-\boldsymbol{J}_{\alpha}^{\mathrm{g}} \cdot \boldsymbol{n}_{\mathrm{lg}}-y_{\alpha}^{\mathrm{g}}\left(j_{\mathrm{w}}^{\mathrm{lg}}+j_{\mathrm{e}}^{\mathrm{lg}}\right)=0,
$$

where $y_{\alpha}^{\mathrm{g}}$ is the mass fraction of $\alpha$-vapor in the gas phase. By assuming constant gas density $\rho^{\mathrm{g}}$, one can approximate $y_{\alpha}^{\mathrm{g}}=c_{\alpha, \mathrm{VLE}} / \rho^{\mathrm{g}}$.

In the droplet, the spatio-temporal liquid composition is governed by the convection-diffusion equations for the liquid mass fractions $y_{\alpha}$, i.e.

$$
\rho\left(\partial_{t} y_{\alpha}+\boldsymbol{u} \cdot \nabla y_{\alpha}\right)=\nabla \cdot\left(\rho D \nabla y_{\alpha}\right)-\boldsymbol{J}_{\alpha} \cdot \boldsymbol{n}_{\lg } \delta_{\lg } .
$$

For simplicity, the composition-dependence of the mass density $\rho$ is approximated by the spatially averaged composition. This assumption has been validated in Diddens et al. [111] and simplifies Eq. (5.6) as well as the momentum equation. At the liquid-gas interface, a source/sink term is imposed via the interface delta function $\delta_{\mathrm{lg}}$. The corresponding flux $\boldsymbol{J}_{\alpha}$ is obtained by the counterpart of Eq. (5.5) for the liquid phase, i.e.

$$
j_{\alpha}^{\lg }-\boldsymbol{J}_{\alpha} \cdot \boldsymbol{n}_{\mathrm{lg}}-y_{\alpha}\left(j_{\mathrm{w}}^{\mathrm{lg}}+j_{\mathrm{e}}^{\mathrm{lg}}\right)=0 .
$$

The mass fraction of the non-volatile anise oil is not solved explicitly, but calculated via $y_{\mathrm{a}}=1-y_{\mathrm{e}}-y_{\mathrm{w}}$.

Due to the latent heat of evaporation $\Lambda_{\alpha}$, the interface is cooled down by the evaporative mass fluxes $j_{\alpha}^{\mathrm{lg}}$. This effect is taken into account by considering the convection-diffusion equation for the temperature $T$, i.e.

$$
\rho c_{p}\left(\partial_{t} T+\boldsymbol{u} \cdot \nabla T\right)=\nabla \cdot(\lambda \nabla T)-\delta_{\lg }\left(j_{\mathrm{w}}^{\lg } \Lambda_{\mathrm{w}}+j_{\mathrm{e}}^{\lg } \Lambda_{\mathrm{e}}\right) .
$$

The mass density $\rho$, the specific heat capacity $c_{p}$ and the thermal conductivity $\lambda$ are different on the individual subdomains. In the droplet, the values furthermore depend on the local mixture composition according to the experimental data of Grolier\&Wilhelm [155] and Yanoet al. [156].

Finally, the velocity $\boldsymbol{u}$ has to be solved. For the moment, the flow in the gas phase is disregarded. The influence of convective mass and thermal transport in the gas phase is discussed later on in section 5.3.4. Due to the micrometer-sized droplet, the Bond number $\mathrm{Bo}$ and the capillary number $\mathrm{Ca}$ are small, i.e. $\ll 1\left(B o<0.08\right.$ and $C a<3 \cdot 10^{-4}$ holds for all droplets discussed in this article). While the former allows to disregard gravitational effects, the latter ensures that the flow in the droplet is dictated by the surface tension. Thus, it is assumed that the droplet is always in an axisymmetric spherical cap shape. Thereby, it is possible to calculate the normal interface velocity 
$\boldsymbol{u}_{\lg } \cdot \boldsymbol{n}_{\lg }$ along the entire interface directly from the evolution of the volume $V(t)$ and the contact angle $\theta(t)$. The volume loss $\dot{V}(t)$ can easily be calculated by integrating the evaporation rates over the liquid-gas interface and by taking the spatially averaged composition-dependence of the mass density into account. The contact angle $\theta$ can be imposed from fitted experimental data for $\theta(t)$ or $\theta(V)$ or, alternatively, obtained from an appropriate model for the composition-dependent contact angle evolution (cf. e.g. Diddens et al. [111]).

The normal interface velocity defines via the kinematic boundary condition the normal flow velocity $\boldsymbol{n}_{\lg } \cdot \boldsymbol{u}$ in the droplet, i.e.

$$
\boldsymbol{n}_{\lg } \cdot \boldsymbol{u}=\boldsymbol{n}_{\lg } \cdot \boldsymbol{u}_{\lg }+\frac{1}{\rho}\left(j_{\mathrm{w}}^{\lg }+j_{\mathrm{e}}^{\mathrm{lg}}\right)=: u_{n} .
$$

With the stress tensor

$$
\mathbf{T}(\mathbf{u})=-\mathbf{p I}+\mu\left(\nabla \mathbf{u}+(\nabla \mathbf{u})^{\mathrm{t}}\right),
$$

the tangential velocity component is subject to the shear stress boundary condition

$$
\boldsymbol{n}_{\mathrm{lg}} \cdot \mathbf{T} \cdot \mathbf{t}_{\mathrm{lg}}-\mathbf{n}_{\mathrm{lg}} \cdot \mathbf{T}^{\mathrm{g}} \cdot \mathbf{t}_{\mathrm{lg}}=\mathbf{t}_{\mathrm{lg}} \cdot \nabla_{\mathrm{lg}} \sigma .
$$

Due to the small viscosity ratio between gas and liquid phase, i.e. $\mu^{\mathrm{g}} \ll \mu$, and the continuous tangential velocity at the interface, the contribution of the shear stress in the gas phase, i.e. the term $\boldsymbol{n}_{\lg } \cdot \mathbf{T}^{\mathrm{g}} \cdot \mathbf{t}_{\mathrm{lg}}$, can be neglected. This assumption is validated later on in section 5.3.4. Since the surface tension $\sigma$ is a function of the composition and the temperature, solutally and thermally driven Marangoni flow has to be expected.

Inside the droplet, a slightly modified Stokes flow is solved:

$$
-\nabla \cdot \mathbf{T}(\mathbf{u})=\mathbf{0} \quad \text { and } \quad \nabla \cdot \mathbf{u}=-\frac{\partial_{\mathbf{t}} \rho}{\rho}
$$

along with the no-slip boundary condition $\boldsymbol{u}=0$ at the substrate $z=0$.

For the implementation of the finite element method, the governing equations are converted to corresponding weak forms [111] and solved with the help of the finite element package FENICS [157].

\subsubsection{Pure water droplet}

To validate the model on the basis of the simplest case, the experimental setup was used to measure the evolution of a $0.63 \mu \mathrm{L}$ pure water droplet at ambient temperature $T_{\infty}=20.4^{\circ} \mathrm{C}$ and relative humidity $\phi=54 \%$. The case of pure water can easily be simulated by setting $y_{\mathrm{w}}=1$ in the model equations. The experimental data is depicted along with the corresponding simulation data in 

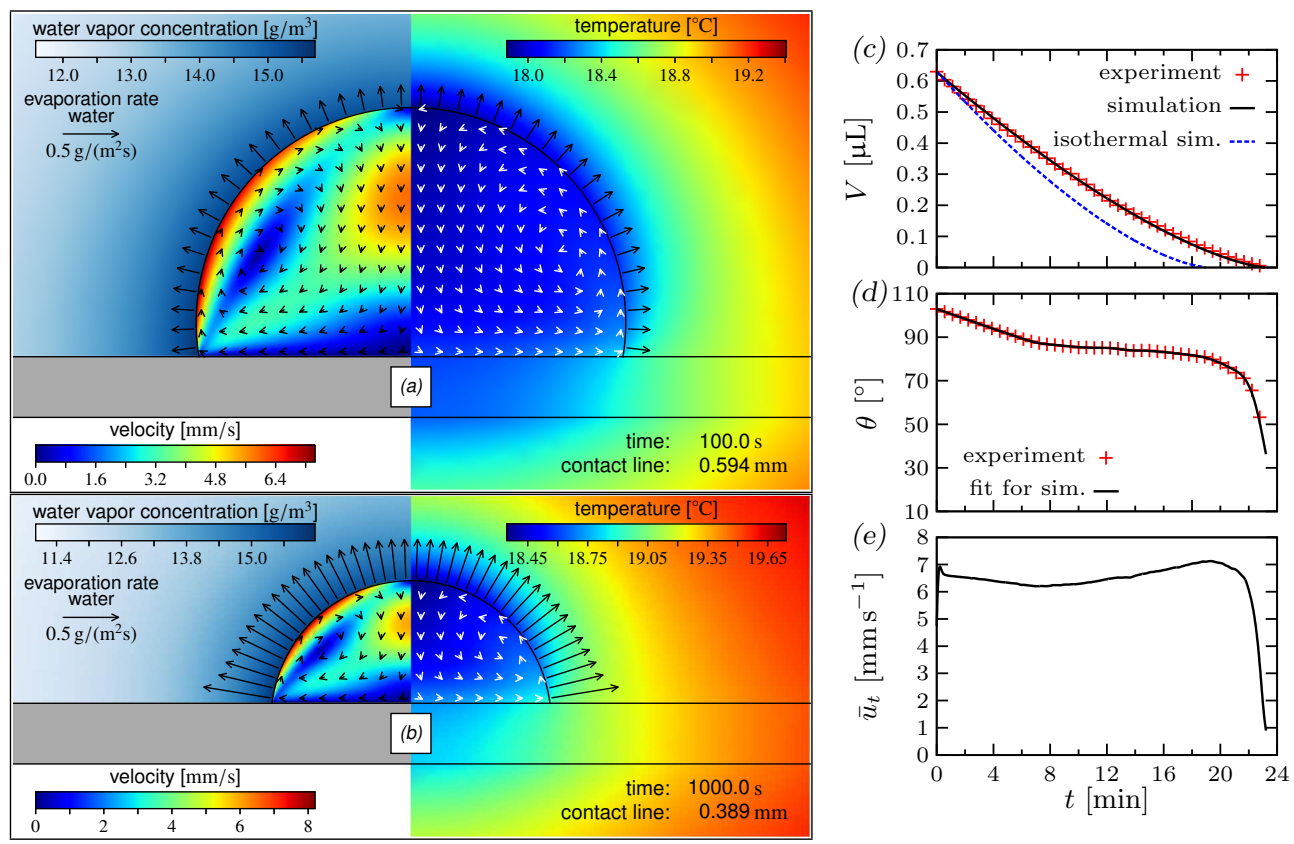

Figure 5.3: (a-b) Snapshots of a pure water droplet with velocity field in the droplet and vapor concentration in the gas phase (left) and temperature field (right) at two different times $t=100 \mathrm{~s}$ (a) and $t=1000 \mathrm{~s}$ (b). A corresponding movie is available as supplementary material. (c) Volume evolution of the experiment and according to simulations with and without considering thermal effects. (d) Contact angle of the experiment and corresponding fit, which is used as input parameter for the simulation. (e) Averaged tangential fluid velocity at the interface.

Figure 5.3. The right side of the snapshots (a) and (b) show the temperature field of the droplet and its surrounding. Due to the thin substrate, the latent heat of evaporation leads to an intense cooling, which can be more than $3 \mathrm{~K}$. From the volume evolution $V(t)$ depicted in (c), it is apparent how the cooling influences the evaporation rate: If the latent heat and the corresponding reduction of the vapor pressure are considered, the numerical simulation perfectly agrees with the experimental data. For comparison, also the corresponding isothermal simulation, i.e. without considering thermal effects, is indicated. This simulation, which resembles the result of the model equation by Popov [32, predicts a noticeably faster drying than the experiment. Thus, even for pure droplets evaporating at room temperature on a glass substrate, thermal effects cannot be disregarded, provided the substrate is thin, i.e. comparable with the droplet size, with air below.

The contact angle evolution in Figure 5.3(d) shows the typical stick-slip behavior, i.e. an initial decrease of $\theta(t)$ at constant contact line radius $r_{\mathrm{c}}$, followed by a constant contact angle with a receding contact line $r_{\mathrm{c}}(t)$. While 
this behavior could be incorporated into the simulation by introducing a receding contact angle, the contact line dynamics of mixture droplets are more rich and complicated to account for with an accurate model. To that end, we have simply fitted the contact angle measured experimentally and used it as an input parameter throughout the simulation.

As can be seen from the left side of the snapshots (a) and (b), the simulation predicts a strong thermal Marangoni flow from the contact line along the liquid-gas interface towards the apex. The corresponding temporal evolution can be inferred from Figure 5.3. (e), where the average tangential fluid velocity at the interface

$$
\bar{u}_{t}(t)=\frac{1}{\int_{\Gamma_{\mathrm{lg}}} r \mathrm{~d} l} \int_{\Gamma_{\lg }} \boldsymbol{u} \cdot \boldsymbol{t}_{\mathrm{lg}} r \mathrm{~d} l
$$

is plotted. The tangent $\boldsymbol{t}_{\mathrm{lg}}$ is defined to point along the interface towards the apex. One can infer that the simulation predicts a persistent thermal Marangoni flow throughout the entire lifetime of the droplet. In experiments, the observed thermal Marangoni flow in water droplets is usually much slower than the theoretical predictions [94]. We discuss this issue later on in section 5.4 .2 ,

\subsubsection{Binary water-ethanol droplet}

As a next step, the model is validated by a binary water-ethanol droplet at ambient temperature $T_{\infty}=21.4^{\circ} \mathrm{C}$ and relative humidity $\phi=55 \%$. Due to practical reasons in the experiment, it took some seconds between the deposition of the droplet and the first snapshot at $t=0 \mathrm{~s}$. Since the average composition changes within this offset time, the initial composition in the simulation has a slightly lower ethanol content $(57.7 \mathrm{wt} \%)$ than specified in section 5.2.1. This particular correction was determined by extrapolating the numerically obtained initial compositional rate of change over the offset time.

Representative snapshots of the simulation are shown in Figure 5.4(a-c). Initially, the droplet is rather flat and both components evaporate with the typical singularity near the contact line. In combination with the fact that water (evaporation rate by arrows on the left side) has a lower volatility than ethanol (arrows on the right side), this leads to an enhanced water concentration near the contact line. The resulting surface tension gradient drives an intense solutal Marangoni flow, which breaks up into chaos due the Marangoni instability of water-ethanol mixtures [158].

In the initial regime, thermal Marangoni flow is almost irrelevant, but the incorporation of the latent heat of evaporation is still important, as it affects again the volume evolution, which is depicted in Figure 5.4(d). As for the case of pure water, the volume $V(t)$ of the simulation agrees perfectly with 

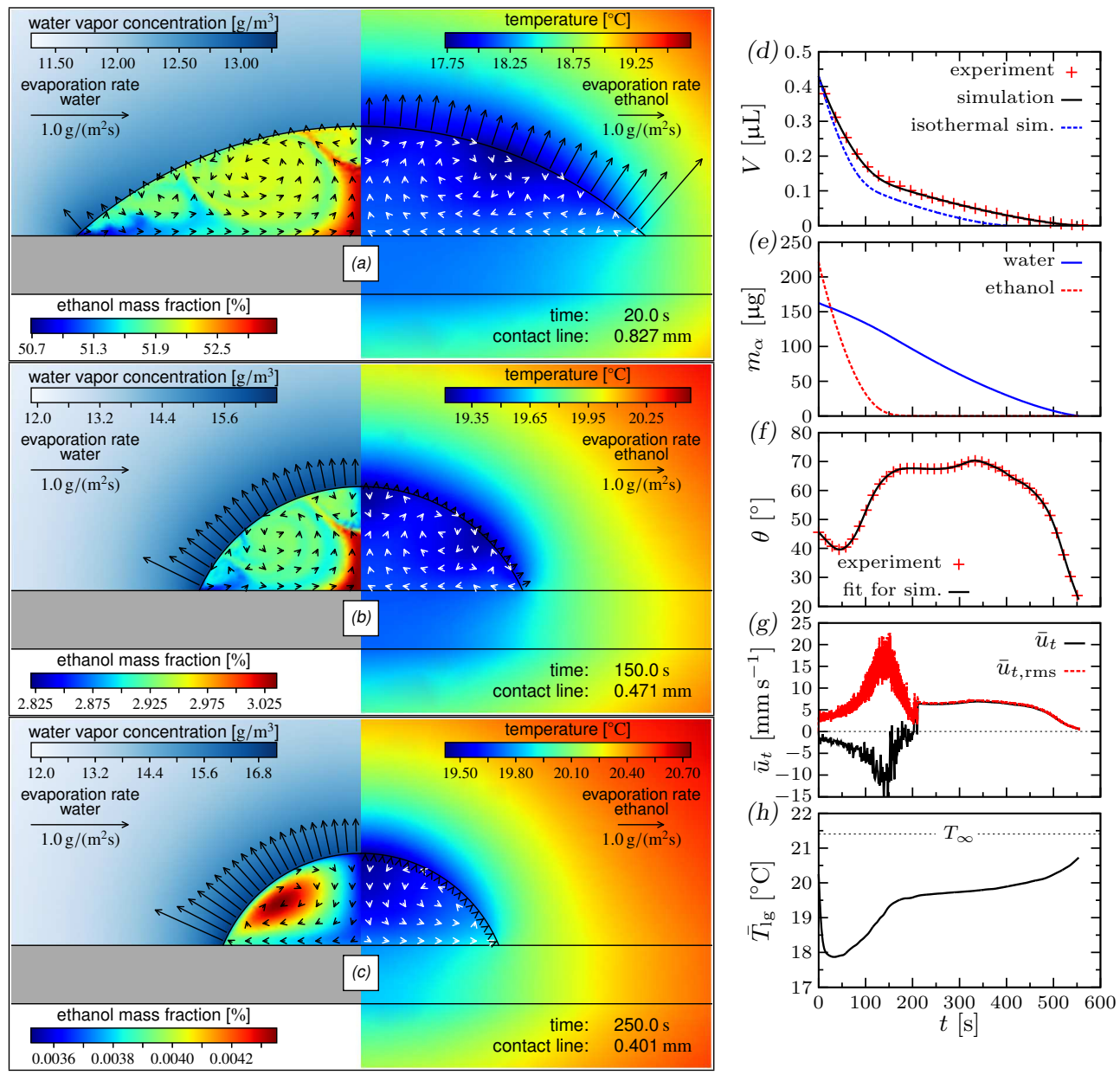

Figure 5.4: (a-c) Snapshots of a binary water-ethanol droplet with ethanol mass fraction $y_{\mathrm{e}}$ in the liquid phase and water vapor concentration in the gas phase (left) and temperature field (right) at three different times $t=20 \mathrm{~s} \mathrm{(a),} t=150 \mathrm{~s} \mathrm{(b)}$ and $t=250 \mathrm{~s} \mathrm{(c)}$. The arrows at the interface indicate the evaporation rates of water (left) and ethanol (right). A corresponding movie is available as supplementary material. (d) Volume evolution according to experiment, full simulation and isothermal simulation. (e) Partial masses of both components in the drop. (f) Experimentally obtained contact angle and corresponding fit for the model. (g) Mean tangential fluid velocity at the interface and its rms-average. (h) Average interface temperature.

the experimental data, but only if the evaporative cooling is considered. In the case of a binary mixture, the evaporative cooling has another effect, namely the suppression of the water evaporation at initial stages: Due to the highly volatile ethanol, the interface is cooled down to a temperature, at which the vapor-liquid equilibrium for the water vapor concentration at the interface almost reaches the level of the vapor concentration in the ambient. At a 
higher relative humidity, this coupling between the evaporation rates and the temperature would even induce an initial condensation of water [111].

The two distinct slopes in the volume curve $V(t)$, which we already observed and explained in Tan et al. [101], result from the preferential evaporation of ethanol in the initial regime followed by the slower evaporation of the remaining water residual at later times. This interpretation is validated by resolving the evolution into the partial masses of water and ethanol as shown in Figure 5.4 (e). It is apparent that the evaporation rate of water, i.e. the slope of the partial mass $\dot{m}_{\mathrm{w}}(t)$, is slightly reduced as long as ethanol is present. This is due to Raoult's law (5.3), but is also caused by the reduction of the vapor pressure due to the additional cooling stemming from the evaporation of ethanol.

The contact angle was again fitted from experimental data and used as input parameter for the simulation. The data for $\theta(t)$ depicted in Figure 5.4(f) reveals an initial decrease due to a pinned contact line followed by an intense increase, which can be understood by considering the composition-dependence of the surface tension in the Young-Laplace equation.

At later times, when virtually all ethanol has evaporated, a flow transition occurs (cf. Figure 5.4(c)): The chaotic solutal Marangoni flow suddenly switches over to regular thermal Marangoni flow as for the pure water droplet of Figure 5.3. The abrupt transition can also be inferred from the plot of the average tangential velocity $\bar{u}_{t}(t)$ in Figure $5.4(\mathrm{~g})$. While the chaotic solutal flow induces a net flow towards the contact line, $\bar{u}_{t}<0$, it suddenly converges to the regular thermal Marangoni flow towards the apex, i.e. $\bar{u}_{t}>0$. Additionally, to estimate the average flow speed at the interface without respecting the local direction, the rms-averaged tangential fluid velocity

$$
\bar{u}_{t, \mathrm{rms}}(t)=\sqrt{\frac{1}{\int_{\Gamma_{\mathrm{lg}}} r \mathrm{~d} l} \int_{\Gamma_{\mathrm{lg}}}\left(\boldsymbol{u} \cdot \boldsymbol{t}_{\mathrm{lg}}\right)^{2} r \mathrm{~d} l}
$$

is plotted in Figure 5.4 (g). It is apparent that the flow is most intense in the limit of rather dilute ethanol. In this region, the compositional surface tension gradient, i.e. $\partial \sigma / \partial y_{\mathrm{e}}$, has its maximum and hence drives the most intense solutal Marangoni flow.

Finally, in Figure 5.4(h), the average temperature of the liquid-gas interface $\bar{T}_{\mathrm{lg}}$ is depicted. Again, cooling can be up to $3.5 \mathrm{~K}$ and is most pronounced during the initial regime, where both components evaporate. Although water has a 2.7 times higher specific latent heat than ethanol, most of the initial cooling is induced by the up to 9 times faster evaporation rate of ethanol. 


\subsubsection{Influence of convective transport in the gas phase}

Before addressing the ternary Ouzo droplet, the flow in the gas phase is investigated. Until now, this flow has been disregarded, but since it generates convective vapor and thermal transport, it can influence the evaporation rates and the temperature field. The flow in the gas phase can most generally be driven by four mechanisms, namely forced convection, natural convection, Marangoni convection and Stefan flow. While forced convection can be ruled out due to the geometry of the experimental setup, the mass density gradient required for natural convection can be caused by a thermal gradient as well as by vapor concentration gradients. Since the temperature at the droplet is lower than the ambient temperature, thermally driven natural convection can be ruled out. To estimate the influence of solutally induced natural convection, we define the solutal Rayleigh number

$$
R a_{\alpha}=\frac{g \beta_{\alpha}\left(c_{\alpha, \mathrm{VLE}}-c_{\alpha, \infty}\right) r_{\mathrm{c}}^{3}}{\nu D_{\alpha}^{\mathrm{g}}},
$$

where $g$ is the acceleration due to gravity, $\nu$ the kinematic viscosity of the gas phase (assumed to be independent of the vapor concentration) and $\beta_{\alpha}=$ $\left(\partial \rho^{\mathrm{g}} / \partial c_{\alpha}\right) / \rho^{\mathrm{g}}$ is the solutal expansion coefficient, which can be calculated by the ideal gas law. The solutal Rayleigh numbers with the largest modulus of the simulations discussed so far read $R a_{\mathrm{w}} \sim 1$ and $R a_{\mathrm{e}} \sim-10$ for water vapor and ethanol vapor, respectively. Note that the different signs originate from the fact that water vapor is less dense than dry air, whereas ethanol vapor is more dense. However, according to Dietrich et al. [127, an influence of solutally driven natural convection on the evaporation rate can be ruled out at these Rayleigh numbers.

Since the tangential velocity is continuous at the interface, the fast Marangoni flow will be also present in the gas phase. Furthermore, the density difference of liquid and vapor leads to a discontinuous jump in the normal velocity component, which constitutes the so-called Stefan flow. To investigate the influence of these effects, the Stokes flow is solved in the gas phase as well, where the normal component of the gas velocity is imposed analogously to $(5.9)$ and the tangential component is imposed to be continuous with its counterpart in the liquid. Furthermore, the convection-diffusion equations (5.1) and (5.8) are augmented with the corresponding convective term in the gas phase and the additional convective vapor transport at the interface, i.e. $c_{\alpha, \mathrm{VLE}}\left(\boldsymbol{u}_{\mathrm{g}}-\boldsymbol{u}_{\mathrm{lg}}\right) \cdot \boldsymbol{n}_{\mathrm{lg}}$, is added to the evaporative mass fluxes.

In Figure 5.5 (a,b), the simulation of the binary water-ethanol droplet of Figure 5.4 with consideration of Marangoni and Stefan flow in the gas phase is depicted. From the velocity field on the right side of the snapshots, it is 
apparent how the Marangoni flow creates vortices in the gas phase, which are initially chaotic and become regular once the ethanol has evaporated. The contribution of the Stefan flow, i.e. the normal velocity jump, is barely visible, since the typical Stefan flow velocity is only about $1 \mathrm{mms}^{-1}$. As can be inferred from Figure 5.5 (c-e), the additional convective transport of vapor and energy has virtually no influence on the volume evolution, the fluid velocity and the interfacial temperature. Hence, disregarding the flow in the gas phase is justified for the discussed cases.

Furthermore, the simulations with flow in the gas phase allow to validate the assumption of omitting the shear stress term $\boldsymbol{n}_{\mathrm{lg}} \cdot \mathbf{T}^{\mathrm{g}} \cdot \mathbf{t}_{\mathrm{lg}}$ in (5.11): The ratio of the shear stresses in the gas phase and in the liquid phase is indeed below $2 \%$.

\subsubsection{Ternary ouzo droplet}

Now that the model has been validated based on a pure water and a binary water-ethanol droplet, we focus on a ternary Ouzo droplet at $T_{\infty}=22.5{ }^{\circ} \mathrm{C}$ and $\phi=40 \%$ in the following. To that end, we took the experimental data of our previous article [101] and performed corresponding simulations with the finite element method described in section 5.3.1. Different from the lubrication theory model used in our previous article [101], the present model takes thermal effects into account and is not subject to the limitation of the lubrication approximation where the contact angle has to be small. In the previous article, we furthermore had to adjust the relative humidity to reproduce the experimental data with the isothermal lubrication theory model in Tan et al. [101].

In Figure 5.6(a-c), the numerically determined onset of the Ouzo effect is depicted (white regions on the left side). As in the experiment, the nucleation of oil microdroplets starts at about $t=20 \mathrm{~s}$ at the contact line. Different from the predictions of the isothermal lubrication theory model [101], the flow in the droplet is chaotic, which leads to a chaotic propagation of the phase separation front through the droplet. At $t \approx 30 \mathrm{~s}$, nucleation can occur everywhere in the droplet.

As it is apparent from the volume evolution curve in Figure 5.6(d), the initial slope matches the experimental results perfectly, but shows deviations at later times. Since the volume evolution of the pure water droplet and the binary water-ethanol droplet perfectly matches the experiments, the disagreement in the ternary case can definitely be attributed to the presence of the third component, namely the anise oil. While it is initially miscible, which is an assumption of the model, the simulation shows perfect agreement in the first regime. At later times, however, the oil ring starts to form, which changes the 

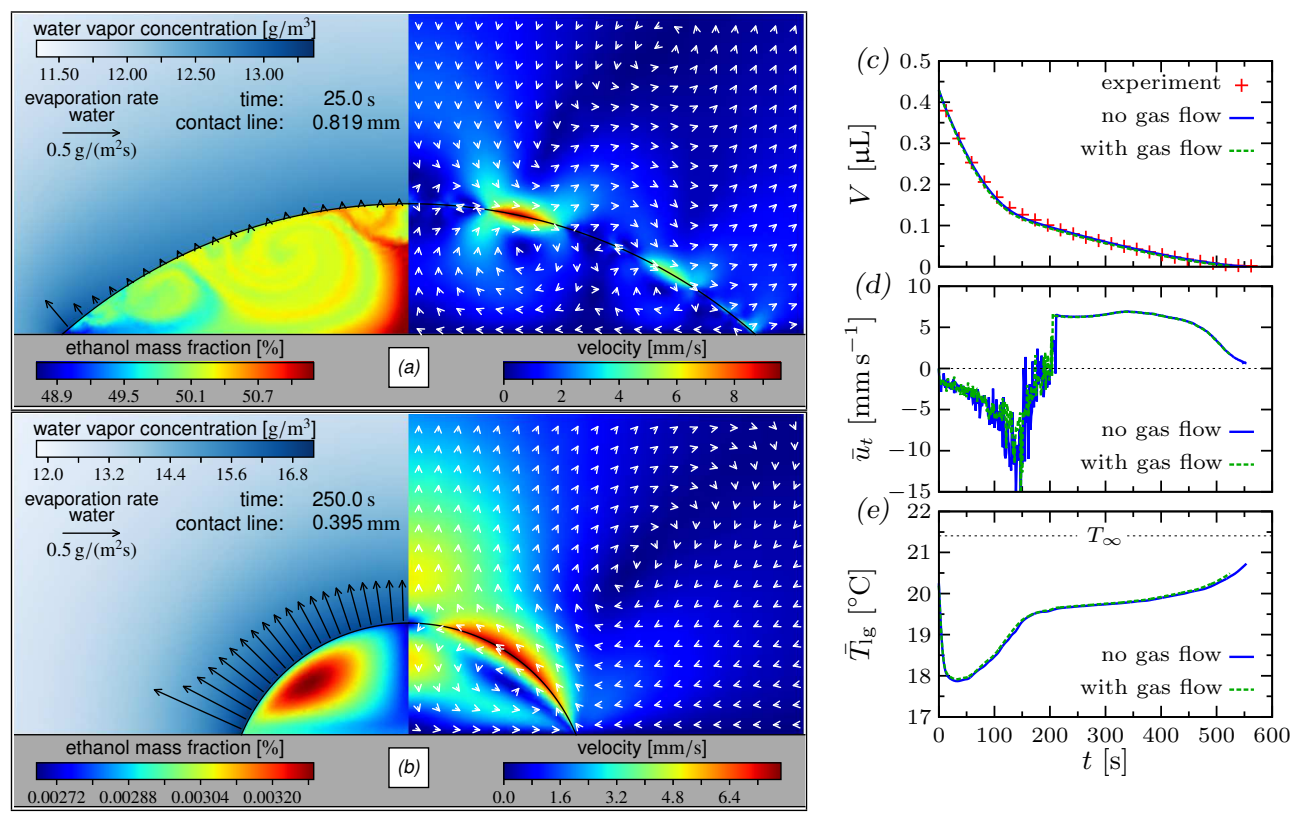

Figure 5.5: (a-b) Same as in Figure 5.4 at two different times $t=25 \mathrm{~s}$ (a) and $t=250 \mathrm{~s}$ (b), but now considering in the calculation and showing in the picture the flow in the gas phase (depicted on the right side). (c-e) Comparison of relevant quantities obtained by simulations with and without flow in the gas phase, revealing hardly any difference.

geometry from the typical spherical cap shape to a more complicated shape, i.e. a water droplet sitting on an oil ring. This leads to a reduction of the water-air interface area and thereby reduces the evaporation rate. The model cannot account for this change in geometry since it always assumes a perfect spherical cap shape and hence predicts a faster evaporation rate. Another possible factor that could contribute to the disagreement is the fact that the anise oil used is not a pure grade of trans-anethole and consists of about $10 \%$ of unknown components [159]. These could also influence the evaporation rate, e.g. by forming a shielding monolayer suppressing evaporation [160].

The other quantities depicted in Figure 5.6(e-h), e.g. the fluid velocity and temperature, are qualitatively similar to the case of the binary water-ethanol droplet of Figure 5.4. While there are temporarily two contact angles in the experiment, i.e. the contact angle $\theta$ between the oil ring and the substrate and the contact angle $\theta^{*}$ of the spherical cap-shaped upper part of the droplet, the present simulation can only take a single contact angle. Here, $\theta^{*}$ was imposed as the contact angle in the simulation during the time the oil ring was present in the experiment. 


\subsection{AXISYMMETRIC INVESTIGATION}
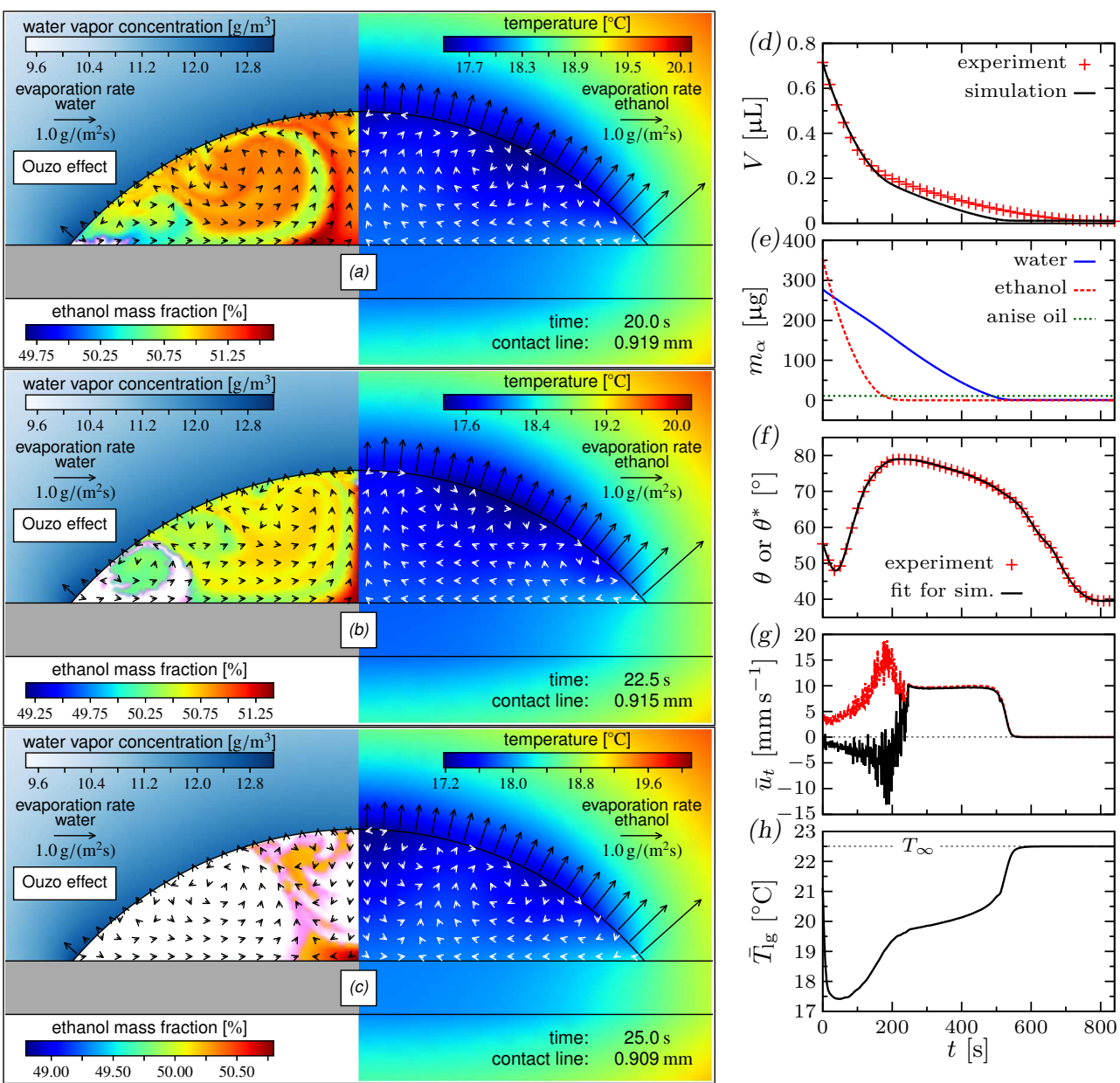

Figure 5.6: (a-c) Snapshots of a ternary Ouzo drop with ethanol mass fraction $y_{\mathrm{e}}$ in the liquid phase and water vapor concentration in the gas phase (left) and temperature field (right) at three different times $t=20 \mathrm{~s}(\mathrm{a}), t=22.5 \mathrm{~s}$ (b) and $t=25 \mathrm{~s}(\mathrm{c})$. Regions where the Ouzo effect occurs are shaded white on the left side. The arrows at the interface indicate the evaporation rates of water (left) and ethanol (right). A corresponding movie is available as supplementary material. (d) Volume evolution according to experiment and the full simulation. (e) Partial masses of all components in the drop. (f) During the evolution, the droplet shape temporarily deviates from a spherical-cap shape due to the presence of the oil ring. During this period, the contact angle in the model was set to the contact angle $\theta^{*}$ of the spherical cap-shaped upper part. Otherwise, the usual contact angle $\theta$ was imposed. (g) Mean tangential fluid velocity at the interface and its rms-average. (h) Average interface temperature. 


\subsection{Axial symmetry breaking}

While the volume evolution predicted by the model has already been compared to the experimental data, a quantitative comparison of the flow velocity remains to be made. However, for the investigation of the initially chaotic flow in the binary water-ethanol and in the ternary Ouzo droplet, an axisymmetric model clearly falls short, since the Marangoni instability will also induce compositional perturbations around the circumference of the droplet and drive Marangoni flow in this direction. This breaking of the axial symmetry for binary water-ethanol droplets has already been observed by Christy et al. [59], Bennacer \& Sefiane [100] and Zhong \& Duan [153]; however, to our knowledge, it has not yet been studied by numerical simulations. In the following, we investigate this aspect of multi-component droplets by comparing experimental micro-PIV measurements with the results from the three-dimensional finite element method. Here, we focus on the binary water-ethanol droplet as depicted in Figure 5.4 for two reasons: On the one hand, the volume evolution shows perfect agreement for this droplet and, on the other hand, the presence of oil microdroplets in the Ouzo droplet hinders the use of the micro-PIV technique as described in section 5.2.3.

\subsubsection{Three-dimensional model}

To investigate the axial symmetry breaking within the framework of the model, it has to be generalized to three dimensions. Mathematically, this generalization from the two-dimensional axisymmetric model to a full three-dimensional variant is an easy step. One just has to transfer the weak forms [cf. 111] to their corresponding three-dimensional equivalents. However, from the perspective of implementation, one faces the challenge of having to treat a free boundary problem. In the axisymmetric model, the movement of the sharp liquid-gas interface was made possible by shifting interfacial mesh nodes and performing a local reconstruction of the mesh whenever necessary to prevent the elements from collapsing. For the interpolation from the previous mesh to the reconstructed mesh, the supermesh method introduced by Farrell et al. [161] was utilized. Both the local mesh reconstruction and the supermesh method constitute a fundamental challenge to be generalized to three dimensions. To circumvent these problems, we focus on a static three-dimensional mesh instead. This approach does not allow to perform a single simulation over the entire lifetime of the droplet, but can still capture the three-dimensional flow statistics for a specific droplet composition. The use of a static mesh is possible, since the typical flow velocity in the droplet is several orders of magnitude larger than the movement of the interface. 


\subsection{AXIAL SYMMETRY BREAKING}
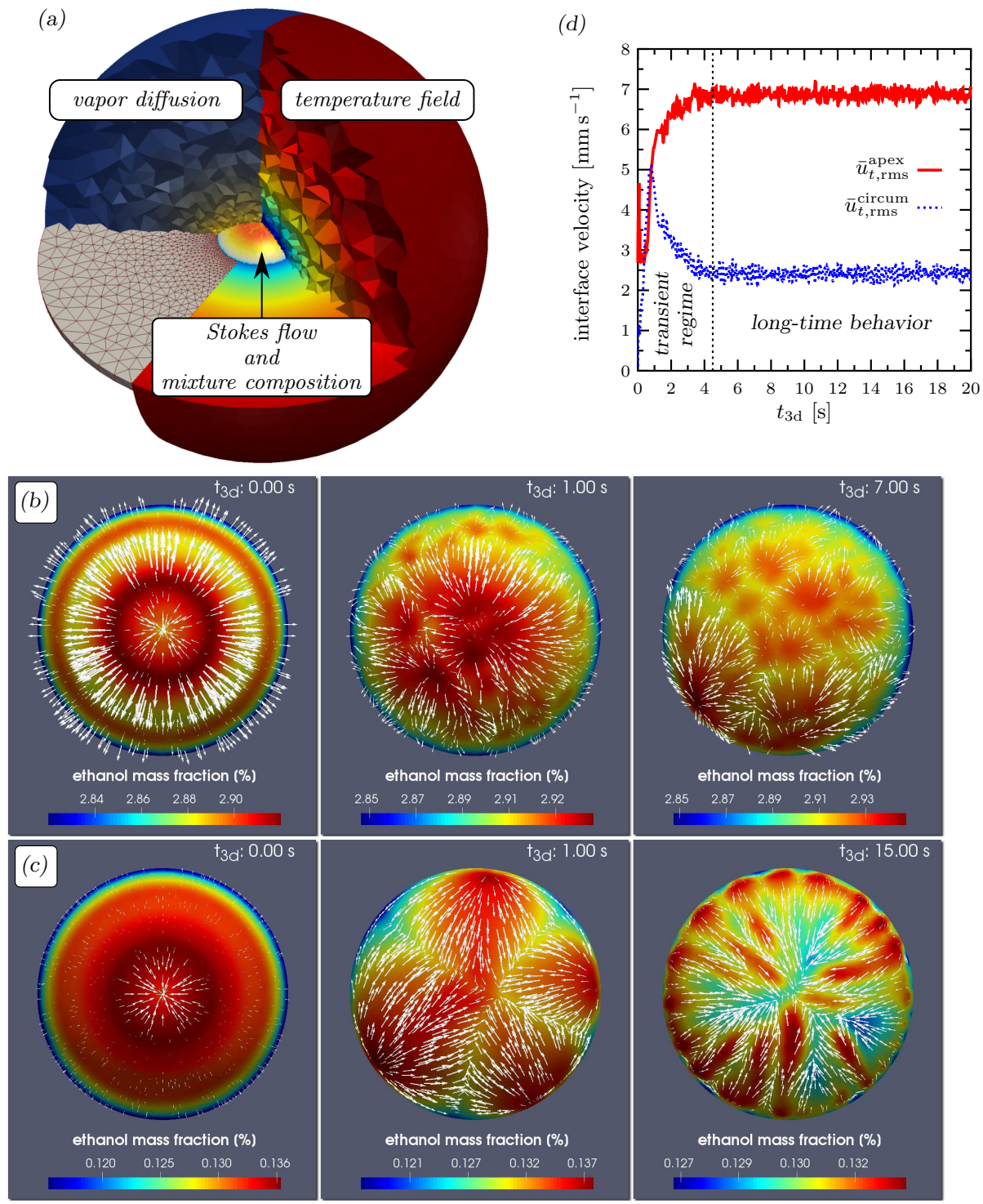

Figure 5.7: (a) View into a mesh for the three-dimensional model. (b) Axial symmetry breaking for snapshot time $t=150 \mathrm{~s}$ at different three-dimensional simulation times $t_{3 \mathrm{~d}}$. The droplet is depicted in top view showing the ethanol mass fraction at the interface. The composition and the tangential fluid velocity at the interface (white arrows) quickly break up into a chaotic, highly non-axisymmetric behavior with a cellular structure. (c) Same as (b), but for the snapshot time $t=200 \mathrm{~s}$. Due to the low ethanol concentration, the break-up is slower. At later times, a cellular pattern can be observed along the rim, which is pulled in filaments towards the apex by thermal Marangoni flow. (d) Evolution of the rms-averaged tangential fluid velocity components $\bar{u}_{t, \mathrm{rms}}^{\text {apex }}$ and $\bar{u}_{t, \mathrm{rms}}^{\mathrm{circum}}$ for the simulation of (c). After a transient regime, the dynamics end up in a chaotic long-time regime with converged mean values and standard deviations. 
The procedure is as follows: For selected individual times $t$ of the axisymmetric simulation depicted in Figure 5.4, we project the axisymmetric data on a corresponding three-dimensional mesh (cf. Figure 5.7(a)). This is used as initial condition for the generalized three-dimensional model, which is integrated over another time $t_{3 \mathrm{~d}}$ to obtain the characteristic three-dimensional flow at time instant $t$. Again, the vapor diffusion equations for the volatile components are solved in the gas phase, the convection-diffusion equations for the composition and the Stokes flow are solved in the droplet, and the temperature field is solved in all domains, including the substrate and the air below it. Since the mesh is static, the kinetic boundary condition has to be modified to $\boldsymbol{n}_{\mathrm{lg}} \cdot \boldsymbol{u}=0$. Furthermore, to capture the characteristic three-dimensional flow at time instant $t$, one has to ensure that the average composition is not changing within the simulation time $t_{3 \mathrm{~d}}$ of the three-dimensional model. This can be achieved by augmenting the convection-diffusion equation 5.6. by a spatially uniform correction term which compensates for the evaporation, i.e.

$$
\rho\left(\partial_{t_{3 \mathrm{~d}}} y_{\alpha}+\boldsymbol{u} \cdot \nabla y_{\alpha}\right)=\nabla \cdot\left(\rho D \nabla y_{\alpha}\right)-\boldsymbol{J}_{\alpha} \cdot \boldsymbol{n}_{\lg } \delta_{\lg }+\frac{1}{V} \int_{\Gamma_{\lg }} \boldsymbol{J}_{\alpha} \cdot \mathrm{d} \boldsymbol{A}
$$

The axisymmetry of the initial condition from the axisymmetric model can break up by the Marangoni instability. Azimuthal perturbations of the composition can arise, yielding to non-axisymmetric solutal Marangoni flow which in turn feeds back to the coupled dynamics of multi-component flow, evaporation and thermal effects. A representative example is shown in Figure 5.7(b) for the snapshot time $t=150 \mathrm{~s}$, which corresponds to the axisymmetric initial condition of Figure 5.4(b). Within a very short three-dimensional simulation time $t_{3 \mathrm{~d}}$, the axial symmetry of mixture composition and Marangoni flow is broken and the system exhibits highly non-axisymmetric dynamics with a cellular structure.

When the ethanol concentration is lower and thermal Marangoni flow becomes relevant, the symmetry break-up is slower. This can be seen from the simulation at snapshot time $t=200 \mathrm{~s}$ depicted in Figure 5.7(c). In an initial transient regime, perturbations arise at the apex and near the contact line, which coalesce to four large cells. With ongoing simulation time, however, new perturbations arise at the contact line, which form small spots with enhanced ethanol concentration. Due to thermal Marangoni flow, filaments of enhanced ethanol concentration are pulled out of these spots towards the apex. The spots near the contact line have a remarkable similarity to the hydrothermal waves in ethanol droplets on heated substrates as reported by Sobac \& Brutin [162]. The fundamental difference is, however, that the hydrothermal waves are a result of an intense thermal Marangoni effect alone, whereas here a combination of thermal and solutal Marangoni flow induces these structures. 
For even lower ethanol concentrations, i.e. for snapshot times $t>225 \mathrm{~s}$, the initial axisymmetry persists, i.e. a breaking of the axial symmetry cannot be observed.

To determine the typical three-dimensional flow characteristics, the simulation time $t_{3 \mathrm{~d}}$ has to be sufficiently long. In particular, we are interested in the long-time regime only, i.e. not in the transient regime as e.g. depicted in the central picture of Figure 5.7 (c). To determine whether the simulation has already reached its long-time behavior, the rms-averaged tangential fluid velocity at the interface is investigated. In order to distinguish between axisymmetric behavior and broken symmetry later on, the rms is split into its two components, namely

$$
\begin{aligned}
\bar{u}_{t, \mathrm{rms}}^{\text {apex }}(t) & =\sqrt{\frac{1}{\int_{\Gamma_{\mathrm{lg}}} \mathrm{d} A} \int_{\Gamma_{\mathrm{lg}}}\left(\boldsymbol{u} \cdot \mathrm{t}_{\mathrm{lg}}^{\mathrm{apex}}\right)^{2} \mathrm{~d} A} \\
\bar{u}_{t, \mathrm{rms}}^{\text {circum }}(t) & =\sqrt{\frac{1}{\int_{\Gamma_{\mathrm{lg}}} \mathrm{d} A} \int_{\Gamma_{\mathrm{lg}}}\left(\boldsymbol{u} \cdot \boldsymbol{t}_{\mathrm{lg}}^{\text {circum }}\right)^{2} \mathrm{~d} A},
\end{aligned}
$$

where $\boldsymbol{t}_{\mathrm{lg}}^{\mathrm{apex}}$ is the interface tangent pointing towards the apex and $\boldsymbol{t}_{\mathrm{lg}}^{\text {circum }}$ is the tangent pointing along the circumference of the droplet. Hence, a nonvanishing value of $\bar{u}_{t, \mathrm{rms}}^{\text {circum }}$ with respect to $\bar{u}_{t, \mathrm{rms}}^{\mathrm{apex}}$ indicates the absence of axial symmetry. A typical evolution of these quantities is depicted in Figure 5.7(d). Initially, the Marangoni instability breaks the axial symmetry, which results in an increasing value of $\bar{u}_{t, \mathrm{rms}}^{\mathrm{circum}}$. After that, it can take several seconds of simulation time $t_{3 \mathrm{~d}}$ until the system enters its long-time behavior. The longtime behavior regime can be identified when both $\bar{u}_{t, \mathrm{rms}}^{\mathrm{apex}}$ and $\bar{u}_{t, \mathrm{rms}}^{\text {circum }}$ show a converged mean value and variance.

The characteristic flow statistics were finally extracted based on at least $t_{3 \mathrm{~d}}=30 \mathrm{~s}$ of long-time behavior for different snapshot times $t$. The resulting data for the tangential fluid velocity components is depicted in Figure 5.8 Initially, the droplet is highly non-axisymmetric with $\bar{u}_{t, \mathrm{rms}}^{\mathrm{apex}} \approx \bar{u}_{t, \mathrm{rms}}^{\text {circum }}$. With ongoing time, ethanol evaporates and since the composition-dependence of the surface tension has its steepest gradient in the limit of dilute ethanol, the flow becomes faster. After a maximum at about $t \approx 130 \mathrm{~s}$, the average velocity decreases again. Until $t=175 \mathrm{~s}$, the characteristic flow pattern is predominantly driven by solutal Marangoni flow. The ethanol concentration at the interface exhibits spatio-temporally chaotic cellular patterns. Moreover, it is possible that a net flow from one side of the rim to the opposing side can build up (e.g. from right to left in the inset at $t=175 \mathrm{~s}$ ). After $t=175 \mathrm{~s}$, i.e. after the local minimum of $\bar{u}_{t, \mathrm{rms}}^{\mathrm{apex}}$, a transition can be observed. The flow is mainly directed towards the apex due to the thermal Marangoni effect, but the dilute ethanol still causes symmetry-breaking perturbations (cf. inset 


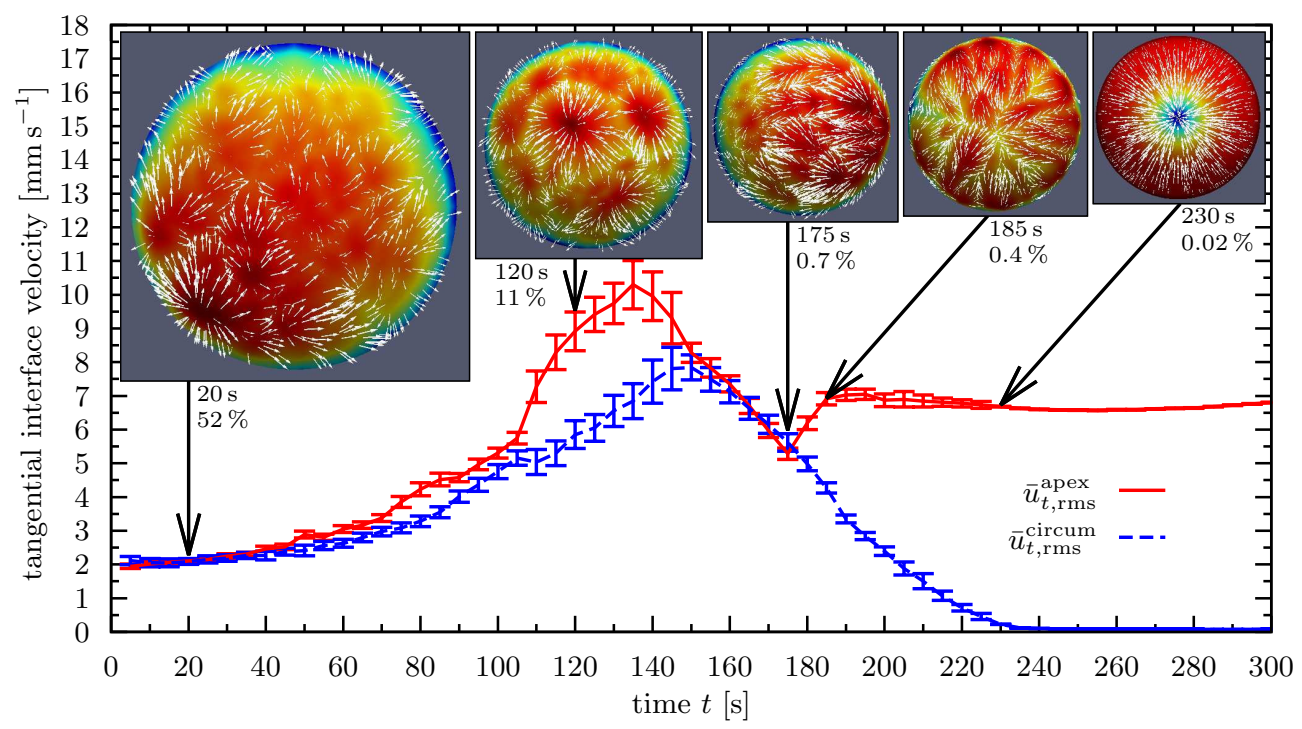

Figure 5.8: Evolution of the rms-averaged tangential velocity components based on the longtime behavior of individual 3d-simulations corresponding to the axisymmetric simulation of Figure 5.4 The error bars indicate the standard deviation and the insets show typical snapshots of the $3 \mathrm{~d}$-simulations at the indicated times. The numbers below the snapshot are the time and the averaged ethanol mass fraction.

at $t=185 \mathrm{~s})$. This characteristic flow pattern has already been discussed in Figure 5.7(c). With ongoing time and vanishing ethanol, the flow gets more and more axisymmetric and regular, which can be inferred by the vanishing value of $\bar{u}_{t, \mathrm{rms}}^{\text {circum }}$.

\subsubsection{Comparison with experiment}

As described in section 5.2.3 micro-PIV measurements were performed during the evaporation of the binary water-ethanol droplet. Thereby, experimental data for the flow in the focal plane, i.e. close to the substrate became available. Since the simulation solves the entire flow field in the bulk, it is possible to extract the corresponding velocity by slicing the three-dimensional mesh at the focal plane and projecting the local velocity vectors onto it. This allows for a direct comparison of experimentally and numerically determined velocity.

Representative snapshots of the experimental micro-PIV measurement are depicted in Figure 5.9(a-f). It is apparent that the axisymmetry is initially broken by the presence of multiple vortices near the liquid-air interface $(a, b)$. With ongoing time, the flow gets more intense and more chaotic up to a maximum at about $t=100 \mathrm{~s}(\mathrm{c}, \mathrm{d})$. Directly after that, a net flow towards the center of the focal plane is building up, which is not perfectly axisymmetric 
but globally it is directed radially inward (e). The flow is slowing down until it completely stops at about $t=140 \mathrm{~s}$ (f).

The data extracted from the numerical simulation is depicted in Figure 5.9 (g1) for comparison. It is apparent how the typical flow is very similar at early times $(\mathrm{g}, \mathrm{h})$, but in the numerics the maximum averaged flow intensity can only be found at $t \approx 150 \mathrm{~s}(\mathrm{i}, \mathrm{j})$, i.e. when the flow has already ceased in the experiments. After this maximum, the flow does not turn inward, but outward instead, which is caused by the thermal Marangoni effect. As long ethanol resides in the droplet, deviations from perfect axisymmetric thermal Marangoni flow can be seen $(\mathrm{k})$. These eventually decrease over time until the flow finally converges to a perfectly axisymmetric intense thermal Marangoni flow. The initial agreement of experiment and simulation can also be inferred from Figure $5.10(\mathrm{a})$, where the averaged velocities in the focal plane are plotted following both methods. At later times, however, the disagreement is obvious.

Since the disagreement is most pronounced at later times, it cannot be attributed to the presence of a binary mixture. In fact, for $t \gtrsim 250 \mathrm{~s}$ the droplet is virtually a pure water droplet, which should show an intense thermal Marangoni flow according to the simulation, which is not observed in experiment. This disagreement between prediction and observation of thermal Marangoni flow in water droplets is well-known 94. At $t=250 \mathrm{~s}$, the numerically obtained temperature difference between contact line and apex reads $\Delta T \approx 0.57 \mathrm{~K}$, which corresponds to a surface tension difference of $\Delta \sigma \approx$ $-0.084 \mathrm{mNm}^{-1}$, i.e. $0.1 \%$ of the averaged surface tension. As already discussed by $\mathrm{Hu} \&$ Larson [94], this small thermally induced difference can be counteracted by a very small amount of surface-active contaminants, which are unavoidable during the experiment.

Therefore, the present scenario, i.e. good agreement at initial stages and large disagreement in the limit of pure water, can presumably be attributed to the presence of contaminants. Since the initial solutal Marangoni instability is intense and chaotic, the contaminants are unable to arrange to a counteracting distribution along the interface. With ongoing time, three mechanisms can contribute to an increase of the overall contaminant concentration at the interface. These are the fact that the droplet and thus the interface are shrinking, a possible ongoing adsorption of contaminants from the gas phase during the evaporation process, and a possibly increasing interface affinity of the contaminants with decreasing ethanol concentration. Once the contaminant concentration is sufficiently high, they start to stabilize the solutal Marangoni flow leading to the almost symmetric radially inward flow as seen in Figure 5.9 (e). Eventually, the thermal Marangoni flow is completely suppressed by the contaminants (cf. Figure 5.9(f)). However, to substantiate 


\section{CHAPTER 5. EVAPORATING PURE, BINARY \& TERNARY DROPS}

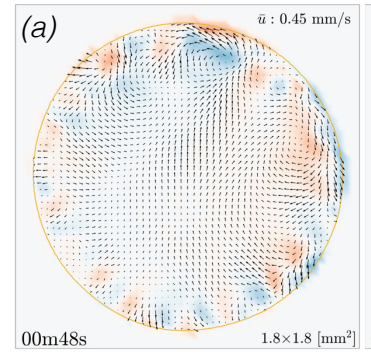

(d) (b)

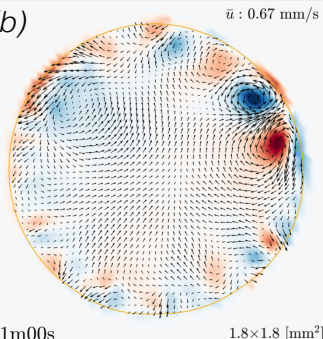

(e) (c)

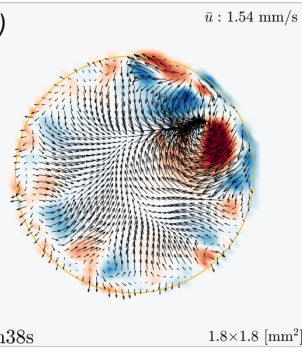

(f) $\bar{u}: 0.04 \mathrm{~mm} / \mathrm{s}$
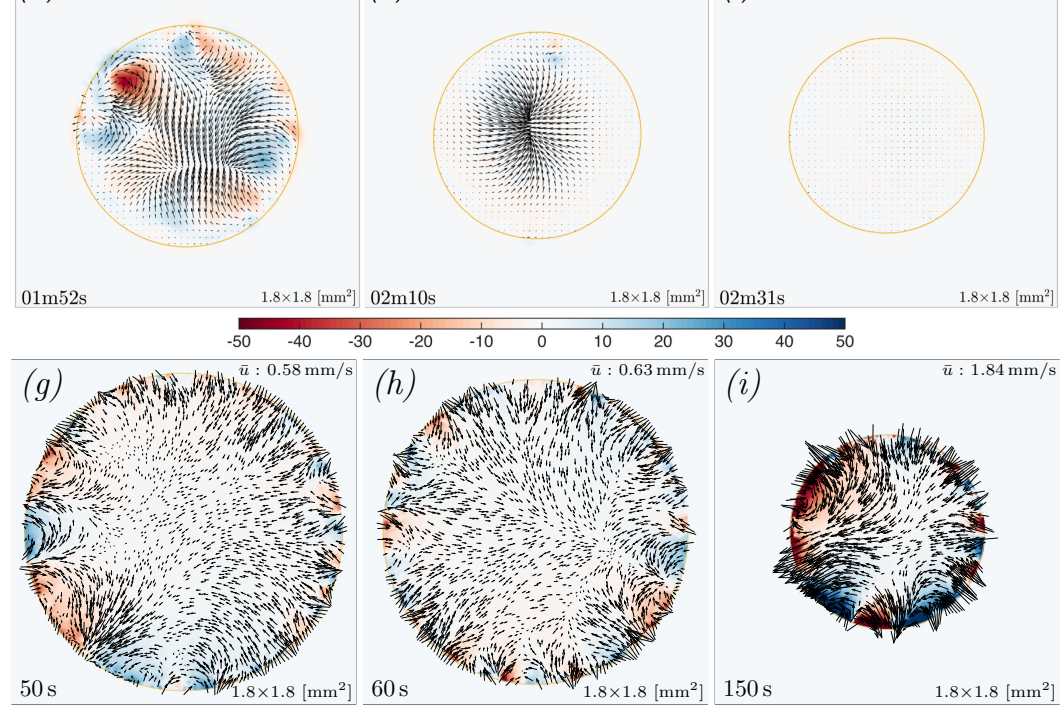

(i) $\bar{u}: 1.84 \mathrm{~mm} / \mathrm{s}$

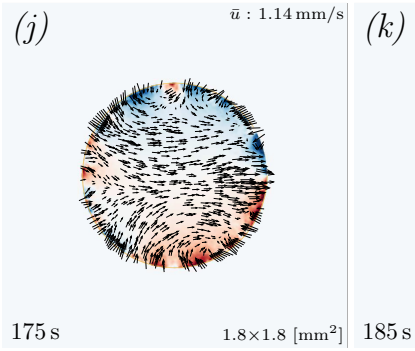

(k)
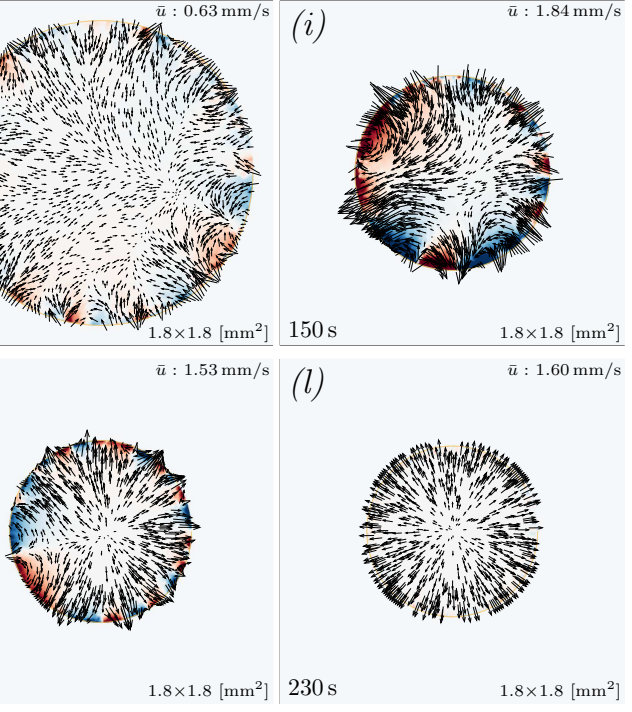

$\bar{u}: 1.60 \mathrm{~mm} / \mathrm{s}$

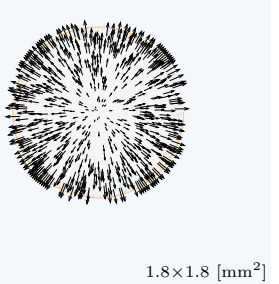

Figure 5.9: (a-f) Experimental snapshots of the velocity field in the focal plane at different instants. The arrows display the local velocity and the vorticity is color-coded. The yellow circle indicates the intersection of the liquid-air interface and the focal plane. (a) Small vortices are present close to the liquid-air interface, breaking the axial-symmetry in the entire droplet. (b) Small vortices can coalesce into a pairs of bigger vortices. (c,d) The overall flow velocity and vorticity increase. (e,f) After a while, the flow has an inward flow pattern (but not perfectly axisymmetric) and finally calms down. (g-l) Velocity in the focal plane as extracted from the numerics. While the simulations shows initially good agreement with the experiment, deviations are present at later times. Note that the color code is the same for both experiment (a-f) and numerics (g-l). Corresponding movies are available as supplementary material. 

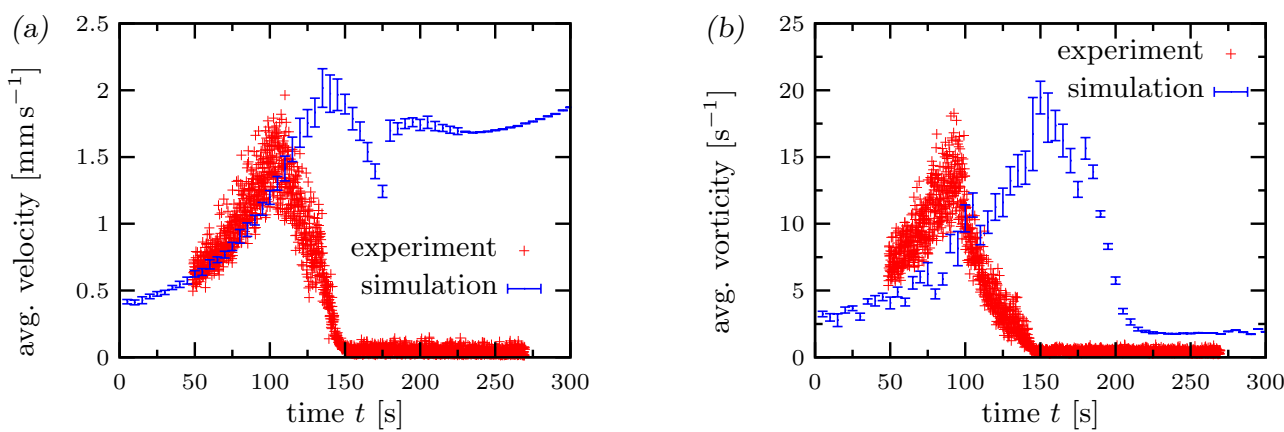

Figure 5.10: Averaged velocity (a) and vorticity (b) in the focal plane based on the experiment and the numerical simulation. The error bars indicate the standard deviation of the numerically obtained quantities.

this scenario, more elaborate experiments, in particular measurements of the contaminant concentration at the interface, or a generalization of the model considering surface-active contaminants would be necessary. It can also not be ruled out that the hydrophobic polystyrene tracer particles influence the interface dynamics.

\subsection{Conclusion}

In conclusion, we have investigated the evaporation of multi-component droplets with numerical and experimental methods. By comparing the results of these methods, we are able to draw the following main conclusions:

Due to the good agreement of the numerical predictions and the experimental data for a pure water droplet and a binary water-ethanol droplet, we have validated the axisymmetric finite element method model of Diddens et $a l$. 111. It has been shown that the quality of the agreement decisively depends on the consideration of the interplay of multi-component evaporation and thermal effects. In particular, it has been shown that even for a pure water droplet the accuracy of the isothermal model of Popov [32] is limited if the substrate is thin. Any noticeable influence of Marangoni flow and Stefan flow in the gas phase on the evaporation has been ruled out for these droplets. While the pure water droplet and the binary water-ethanol droplet are in perfect agreement with the experiment, the simulation of the ternary Ouzo droplet initially shows good agreement, including the onset of oil nucleation, but exhibits a faster evaporation of the remaining water residual than in experiment. This issue can presumably be attributed to the presence of the oil ring, i.e. a geometric deviation from the typical spherical cap shape, and which was not included in the present model. 
To experimentally investigate the flow in the binary water-ethanol droplet, micro-PIV measurements have been performed. Since the flow is clearly nonaxisymmetric as long as ethanol is present, the model had to be generalized to a full three-dimensional version. While the data is initially in good quantitative agreement, deviations between experiment and simulation can be found at later times. In particular, the simulation shows an intense thermal Marangoni flow once pure water remains, whereas the micro-PIV measurement shows no flow at all. Since all other aspects, i.e. composition-dependent liquid properties and thermal influences, are considered in the model, the mismatch can only be attributed to the presence of surface-active contaminations. This observation encourages the development of even more elaborate models which will take the role of surfactants into account and the conduction of novel experiments which try to completely exclude any contamination. 



\section{Part II}

Colloidal ouzo drops 



\section{6}

Porous supraparticle assembly in colloidal ouzo drops*

The assembly of colloidal particles from evaporating suspension drops is seen as a versatile route for the fabrication of supraparticles for various applications in catalysis, environmental pollution management, diagnostics, and material science. However, the assembly is hindered from the interaction of the particles with the substrate, as pinning effects lead to uncontrolled shapes of the emerging supraparticles. Liquid-repellent surfaces minimize line pinning, however, they are known to be high-cost and fragile, and their practical application is limited by the difficulty of the supraparticle collection. Here we demonstrate how the pinning problem can be overcome by self-lubrication. The colloidal particles are dispersed in ternary drops, here consisting of water, ethanol, and aniseoil. As the ethanol evaporates, oil microdroplets form ("ouzo effect"), leaving behind the colloidal particles in the aqueous phase. Later the oil microdroplets become jammed, eventually, coalesce and form an oil ring. The water drop with the dispersed colloidal particles and dispersed oil microdroplets is sitting on the oil ring ("self-lubrication"). Then the water evaporates, leaving behind a porous supraparticle, which easily detaches from the surface. The dispersed oil microdroplets act as templates, leading to multi-scale, fractal-like structures inside the supraparticle. Employing this method, we could produce a large number of supraparticles with tunable shapes and high porosity on hydrophobic

\footnotetext{
${ }^{*}$ Based on: H. Tan, S. Wooh, H.J. Butt, X. Zhang and D. Lohse, Porous supraparticle assembly through self-lubricating evaporating colloidal ouzo drops, Submitted.
} 
surfaces. Combing our new method with piezoacoustic inkjet technology opens the way to mass production of controlled porous colloidal supraparticles.

\subsection{Introduction}

Supraparticles (SPs) refer to three-dimensional macroscopic structures by selfassembly of colloidal (micro-) nanoparticles [163 166]. Such particles have been identified as prominent candidates for a wide variety of modern applications like catalysis [167, 168, catalytically active particles [169, adsorbents in environmental pollution management [170, 171], diagnostics [172], chromatography [173], photonics [163, 174], barcodes [175], biomedical delivery [172], and sensing [176, 177]. Supraparticle fabrication by drying microlitre colloidal dispersion drops on surfaces has been extensively investigated in last few decades because of its versatility, operability, energy-efficiency, and potential scalability [163, 165, 178]. Through controlling evaporation rates [166], adding electric or magnetic fields [165, 179, 180], adjusting $\mathrm{pH}$ or ionic strength of dispersion [181, 182], or regulating the surface properties of (multi-) particles and substrate [84, 168, 183 185], the generated SPs can acquire many impressive features, including high surface-to-volume ratio, long-range order, and periodicity at the mesoscale [163, 167, 174, 186, 188].

However, the strong adhesion between the colloidal dispersion drops and the surface hinders this promising technique. Since evaporating colloidal drops normally suffer from a pinned contact line, a capillary flows inside the drop arise and carry the colloidal particles to the edge, leading to a ring deposition, i.e., the so-called "coffee ring effect" [38]. Up to now, the feasible way to suppress the coffee ring effect for supraparticle synthesis is to employ super liquid-repellant surfaces by reducing the initial contact area of the colloidal drops [167, 183. These special surfaces, however, are high-cost and fragile. Besides, it is difficult to achieve a complete detachment of the supraparticles from the surfaces. Another potential way to overcome the pinning effect is to partially submerge the colloidal drops on a lubricated oil layer on the substrate or use a lubricant-impregnate surface, but the mutual attraction of the floating drops through the so-called "Cheerios effect" hinders controllability of this method [180, 189].

In this paper, we will employ the so-called "ouzo effect" to prevent pinning of the evaporating drops by what we call self-lubrication. "Ouzo" is the Greek anise-flavored aperitif, mainly consisting of water, ethanol, and anise-oil. As recently found [101, in evaporating ouzo drops phase separation occurs due to the preferred evaporation of ethanol and the resulting lower oil solubility ("ouzo effect"), preferentially at the contact line, where oil droplets first form. 


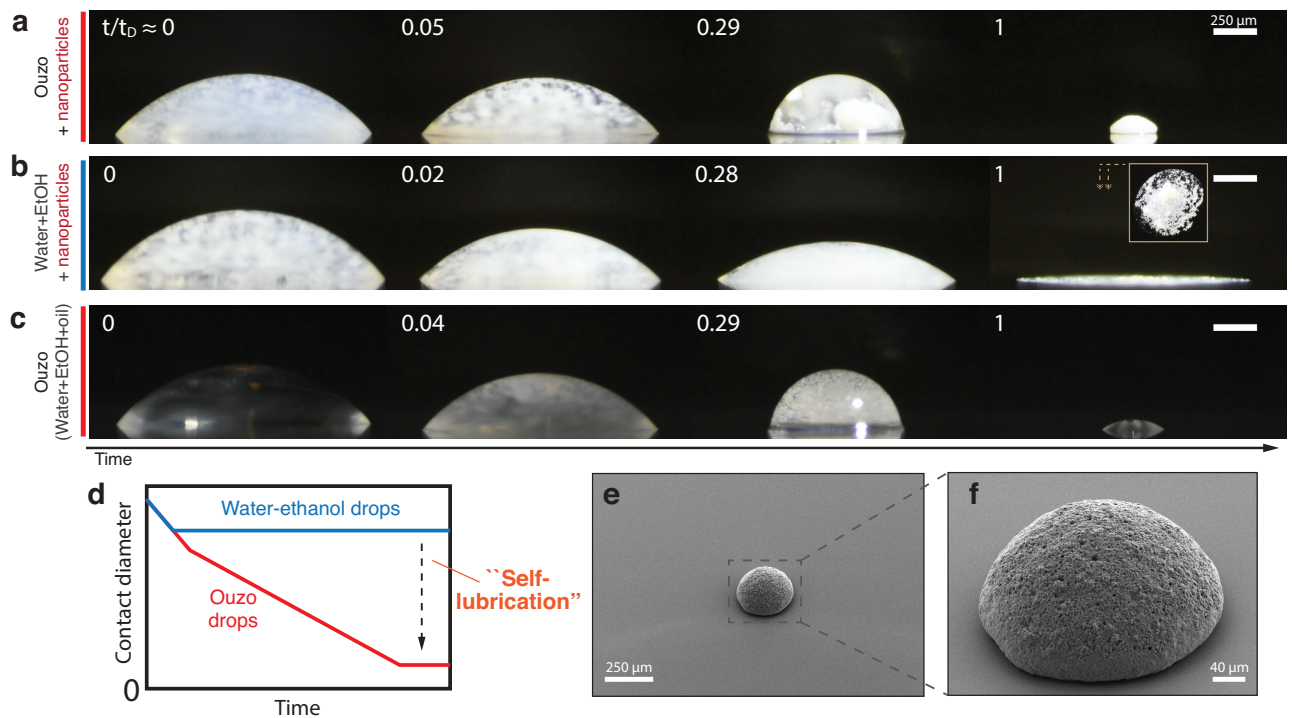

Figure 6.1: Supraparticles self-assembly by drying drops of ouzo suspensions on hydrophobic surfaces. a, Snapshots of the evaporation of a drop of ouzo suspensions. The contact diameter of the drop smoothly decreased during the whole process, and ultimately with a supraparticle generated, as displayed in SEM photographs $(\mathbf{e}, \mathbf{f})$; $\mathbf{b}$, The evaporation of a sessile drop of water-ethanol suspensions with the same water-ethanol-nanoparticle proportion (without anethole). The shrinkage of the contact diameter stopped soon and no supraparticle formed in the end; c, The evaporation of an ouzo drop with the same water-ethanol-anethole proportion (without nanoparticles), which displays the same dynamical evolution as panel (a); d, A schematic illustration of the variation of contact diameter. In cases (a) and (c), drops achieve a much smaller final contact diameter (red line) than case (b) (blue line) as a consequence of the addition of the little anethole, which we call "self-lubrication." The time $t$ is nondimensionalized by the depletion time $t_{D}$. Scale bars (a-c), $250 \mu \mathrm{m}$.

Inspired by this phenomenon, we propose a reliable, robust and cost-efficient method for supraparticle fabrication by employing a ternary liquid with appropriately chosen mutual solubilities ("ouzo" solution) in evaporation-driven particle assembly, which enables us to produce highly porous supraparticles with a tunable shape on commonly-used hydrophobic surfaces.

\subsection{Methods and results}

The method is achieved by using the ternary liquid, here consisting of milli-Q water (39.75 vol\%), ethanol ( $59.00 \mathrm{vol} \%)$, and a slight amount of trans-anethole $(1.20 \mathrm{vol} \%)$ ("ouzo" solution), as the suspension medium of $\mathrm{TiO}_{2}$ nanoparticles $(0.05 \mathrm{vol} \%)$. We deposited a drop of $0.5 \mu \mathrm{L}$ ouzo suspension on a hydrophobic trimethoxy(octadecyl)silane (OTMS)-glass surface. A camera recorded the drop evaporation from the side (Fig. 6.1 $)$. During the drying, an oil ring 
emerged under the colloidal drop [101]. After that, the drop shrank on the surface without pinning. After complete evaporation of first the ethanol and then the water, a supraparticle emerged (Fig.6.1p, f).

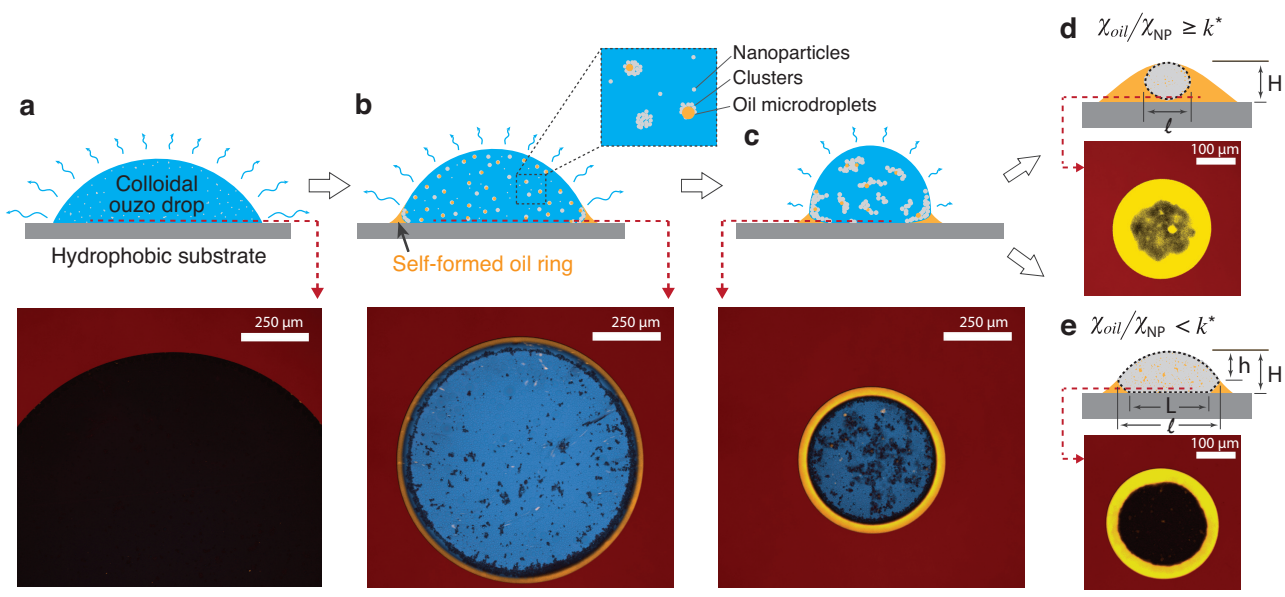

Figure 6.2: Schematic illustrations of supraparticles formation by self-lubrication effect, and the corresponding confocal photographs. Colour indications under a confocal microscope: yellow, oil; blue, water/ethanol; black, clusters of nanoparticles; red, substrate. a, Initial state of evaporating drops of ouzo solution with well-dispersed nanoparticles. High nanoparticle concentration causes the black appearance of the drop under confocal; $\mathbf{b}$, Prevention of nanoparticle deposition at the contact line. The self-formed oil ring thanks to the ouzo effect prevents a pinned contact line and equips colloidal drops with high mobility and low hysteresis. Nanoparticles aggregate and oil microdroplets nucleate on them; c, The shrinkage of the oil ring, which sweeps nanoparticles/clusters from the substrate, preventing sedimentation. After the evaporation of ethanol and water, the generated supraparticles either float on the residual oil (panel $\mathbf{d}$ ) or sit on the substrate (panel $\mathbf{e}$ ), depending on the relation of volume between the supraparticle and residual oil. All the confocal photographs were from a horizontal scanning just above the substrate.

We performed a control experiment (Fig.6.1p) by evaporating a waterethanol-nanoparticle drop (no oil contained, i.e., a binary drop), with the same proportion of water, ethanol, and nanoparticle on the same substrate. In this case, no self-lubricating oil ring could form, and the nanoparticles deposited on the surface with various deposition patterns [153, 190]. In a second control experiment, we evaporated an ouzo drop without dispersed nanoparticles (Fig.6.1 k). It had the same characteristics during evaporation as the case with all ingredients in Figure 6.1 a. The comparison of these three cases demonstrates that the self-formed oil ring plays a crucial role for the characteristics of the decrease of the contact diameter (illustration Fig.6.1 d). The oil ring lubricated the colloidal drop during the self-assembly of supraparticles. Therefore we call this process "self-lubrication" (Fig.6.2. 
We further studied the dynamics of the self-lubrication process and the supraparticle self-assembly with laser scanning confocal microscopy. The formation of the oil ring is followed by conducting a series of horizontal scans $\approx 10 \mu \mathrm{m}$ above the substrate. Perylene (for oil) and rhodamine $6 \mathrm{G}$ (for aqueous) were added into the solution to distinguish different phases: Blue, yellow, black, and red in the confocal images of Figure 6.2 represent the aqueous solution, the phase-separated oil, the nanoparticles (clusters), and the substrate, respectively. Initially, the colloidal ouzo drop appeared black due to the dispersion of high concentration nanoparticles (Fig.6.2 a). The blue color of the solution became visible once nanoparticles started aggregating (inset Fig.6.2 b). The nucleated oil microdroplets attached to nanoparticles (clusters) due to the preference of heterogeneous nucleation as compared to homogeneous nucleation. Next, after the microdroplet nucleation, further nanoparticles will attach to the oil-aqueous interface [191]. Thanks to the presence of the oil ring from the coalescence of surface oil microdroplets, nanoparticles (clusters) were restrained from accumulating at the air-oil-substrate contact line (redyellow boundary line in Fig.6.2p). Driven by evaporation, the colloidal drop contracted radially and forced the oil ring to slide inwards (Fig.6.2 ). The drop contraction led to the nanoparticles assembly forming three-dimensional structures. Here, surface tension prevailed over gravity, as the small drops have small Bond number $B o=\rho g L^{2} / \sigma \sim 10^{-1} \ll 1 . \rho$ is the density of the drop solution $\left(\sim 1000 \mathrm{~kg} / \mathrm{m}^{3}\right), g$ the gravitational acceleration, $L$ the characteristic size of the drop $(\sim 0.5 \mathrm{~mm})$, and $\sigma$ the surface tension (water/trans-anethole interfacial tension [136] 24.2 $\left.\mathrm{mN} \mathrm{m}^{-1}\right)$.

The shrinkage of oil ring causes the levitation of the colloidal drop, and the final geometry of the supraparticle is affected. The ridge of the oil ring arched the colloidal drop (Fig.6.2 ). The inner ridge of the oil ring acted as the lower half of the dynamical mold for supraparticle self-assembly, while the liquid-air interface formed the upper half. Consequently, the oil wetting ridge affects the shape of the formed supraparticles. By changing the geometry of the oil wetting ridge, we could obtain different configurations of the mold and thus the different morphologies of the generated supraparticles (Fig.6.2 de).

We controlled the shape of the generated supraparticles by varying the ratio of the oil volume fraction $\chi_{\mathrm{oil}}$ to the nanoparticles volume fraction $\chi_{\mathrm{NP}}$ in the initial colloidal solution. The full parameter space is shown in Figure 6.3 , giving quantitative information on the final geometry (Fig.6.3 b) and the porosity (Fig.6.3 ) of the supraparticles. The ethanol-to-water volume ratio is 6:4 and the black dashed lines in the parameter space represent different oil-to-nanoparticle ratios $\chi_{\mathrm{oil}} / \chi_{\mathrm{NP}}$. For each data point with a prepared solution composition, the initial profiles of the drops and the final profiles of 

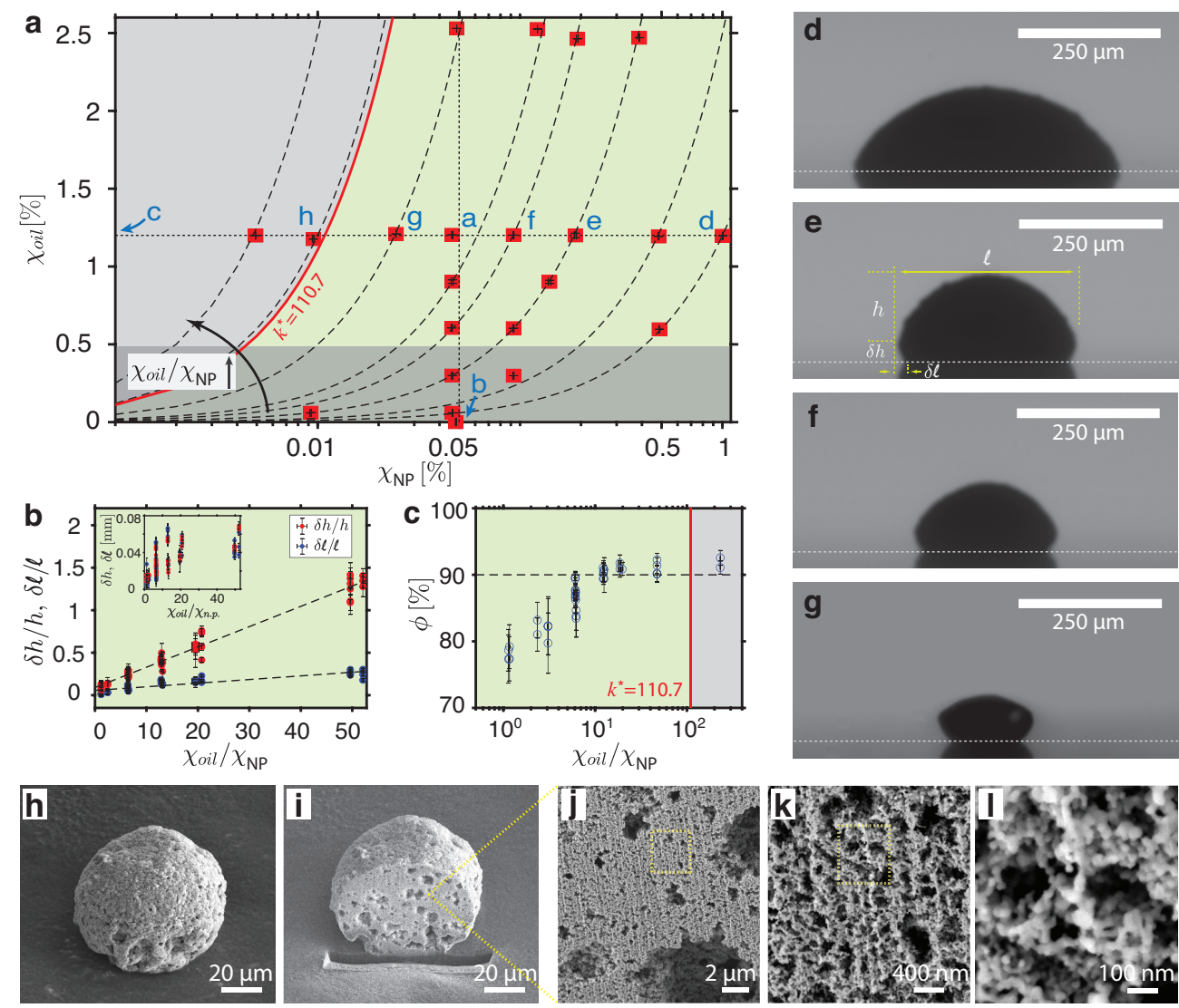

Figure 6.3: Supraparticles with tunable shape and high porosity. a, Parameter space showing the initial oil volume fraction $\chi_{\mathrm{oil}}$ and nanoparticles volume fraction $\chi_{\mathrm{NP}}$ of colloidal drops in different cases with a same ethanol-to-water ratio (3:2). The calculated red solid line $\left(\chi_{\mathrm{oil}} / \chi_{\mathrm{NP}}=110.7\right)$ divides the space into a high (grey) and a low (green) oil-to-nanoparticle ratio regions. b, Both height and depth of the shrunken region (lower part) of supraparticle, nondimensionalized by the height and depth of the upper part, are proportional to the oilto-nanoparticle ratio in the green region. Profile photographs show the diverse shapes, from a spherical-cap shape (d), to a mushroom-like one (e,f), and to a cupcake-like one (g), as the increase of the ratio. In the high ratio region, supraparticles have a ball-like shape (SEM image $\mathbf{h}$ ). c, the calculated porosity $\phi$ of supraparticles ranges from $78 \%$ to $92 \%$. i, Cross-section of the supraparticle in panel $\mathbf{h}$ obtained by FIB cutting illustrates the highly porous structure inside. Panels $(\mathbf{j}-\mathbf{l})$ are a sequence of 3 zooms into the inner structure. The horizontal white dotted lines in panels $(\mathbf{d}-\mathbf{g})$ indicate the substrate position. The shadows below the lines are reflections. The temperature and relative humidity during experiments was $20-23{ }^{\circ} \mathrm{C}$ and $35-50 \%$, respectively. 
the supraparticles (after the depletion of oil) were captured by a grey-scale camera from the side, see Figure6.3 $\mathrm{d}-\mathrm{g}$. These images reveal that the oilto-nanoparticle ratio determines the supraparticle shape. When the volume fraction of oil greatly exceeded the volume fraction of nanoparticles, more spherical supraparticles formed (Fig.6.3h). For less oil, the supraparticles assumed a more flat, oblate shape (Fig.6.3 d-g). Data points a, b $\left(\chi_{\text {oil }}=0\right)$, and $\mathrm{c}\left(\chi_{\mathrm{NP}}=0\right)$ represent the oil and nanoparticle concentrations in the three cases displayed in Figure6.1 a, b, and c, respectively.

We defined the geometric characteristics of the non-ball-like shape by the height and the depth of the dent of the oil ridge, i.e., $\delta h=H-h$ and $\delta l=l-L$ (annotations in Fig.6.3e). This geometrical information was extracted through imaging analyses with a self-made MATLAB program, assuming axial-symmetry. The data in Figure 6.3 b show that both dimensionless height $\delta h / h$ and the dimensionless depth $\delta l / l$ increase monotonously with increasing oil-to-nanoparticle ratio. The inset shows the dimensional data. The monotonic dependence reflects the fact that the oil wetting ridge sculpts the supraparticles. High oil ratios lead to a prominent oil wetting ridge, which causes a noticeable dent in the formed supraparticles.

Ball-like supraparticles are achievable when the oil to nanoparticle ratio is high enough to have developing supraparticles submerged in the oil phase. A cohesive force of the interface layer between the surrounding oil and the submerged drop pulled the developing supraparticle into spherical shapes. Thus ball-like supraparticles are generated, as displayed in the SEM image of Figure 6.3 h. The critical oil-to-nanoparticle ratio $k^{*}$ to have ball-like supraparticles can be estimated by assuming a spherical-cap oil drop with a developing supraparticle submerged inside. The developing supraparticle is in its upper limit size, which is equal to the height $H$ of the oil drop. The residual water fills the porous structure. With these assumptions, we obtained (see supplementary material) $k^{*}=\left(3 \cot ^{2} \frac{\theta_{\text {oil }}}{2}\right) /(1-\phi)$, where $\phi$ is the porosity of the supraparticle, and $\theta_{\text {oil }}$ the contact angle of oil on the surface. Given $90 \%$ porosity and an advancing contact angle of $55^{\circ}$ as found in our measurements, the calculated value is 110.7 (solid red lines in Fig.6.3 ac). This line divides the parameter space into the grey region of ball-like supraparticles and the green region of supraparticles in diverse shapes, being consistent with our observations.

The observed very high porosity of $90 \%$ and beyond is another prominent feature of the supraparticles. We calculated this porosity based on the initial volume of the colloidal drops, with the known nanoparticles concentrations and the final size of the supraparticles. The calculated porosity data shown in Figure 6.3p range from $77 \%$ to $92 \%$ and monotonically increase with the 
oil-to-nanoparticle ratio. The nucleated oil microdroplets in bulk provided a significant contribution to the porosity of the supraparticles. Due to capillary forces, particle network formed among the microdroplets [191], which was also observed in our confocal image Figure 6.2k. Consequently, empty cells were left behind after all the liquids diffused out, dramatically increasing the porosity of the generated supraparticles. Increasing the oil-to-nanoparticle ratio increases the volume of the empty cells, so the porosity of the supraparticles increases (Fig.6.3 ). The limitation of the porosity (92\%) is that, during the contraction of the developing supraparticle, the oil microdroplets merged up gradually and part of them absorbed into the oil ring [101].

The inner structure of the supraparticles reflects the explanation above to the high porosity feature. To reveal this high porosity on all length scales in the interior of the supraparticle, we used the focused ion beam cutting technique to analyze the supraparticle: In Figure 6.3h, slide-by-slide cuts reveal the inner structure. Figure 6.3 displays an exemplary cross-section of the supraparticle. It reveals a multi-scale, fractal-like interior structure, and clearly shows that around half of the particle volume are micron-size holes (Fig.6.3j). The rest contains many smaller holes of sub-micron size (Fig.6.3k). Nanoparticles joint together forming nanoparticle branches and mesopores (nanometer size) (Fig.66.3.). These holes of (sub-) micron size originate from the nucleated oil microdroplets in the colloidal ouzo drop, as the nucleating oil microdroplets act as cells devoid of (clusters of) nanoparticles during the supraparticle aggregation.

An engineering benefit of this method is the ease of scalability of the supraparticle fabrication. To give a demonstration to this advantage, we built up a simple system in our laboratory (Fig.6.4 a), which enables the automatic production of drops of similar size on trichloro(octadecyl)silane (OTS) or OTMS surfaces at rates of 20 drops per minute. Few minutes after the drop deposited, the supraparticles synthesis achieved. The supraparticles harvest was carried out by merely immersing the supraparticle-attached surface inside ethanol, and shaking them off with ease. As a result, we had the supraparticles stored in liquid for future usage, and the surface was clean and ready for the next fabrication process. After several cycles, the supraparticle suspension is available. The self-lubricant layer and the complete detachment of the supraparticles increase the flexibility of supraparticle fabrication. Masses of supraparticles without controlled sizes can be fabricated through spraying the colloidal ouzo solution on the surface.

By applying different types of nanoparticles or multiple types of nanoparticles, we produced different kinds of supraparticles for demonstration. Figures $6.4 \mathrm{~b}$-f are the SEM photographs of a large quantity of the generated supra- 

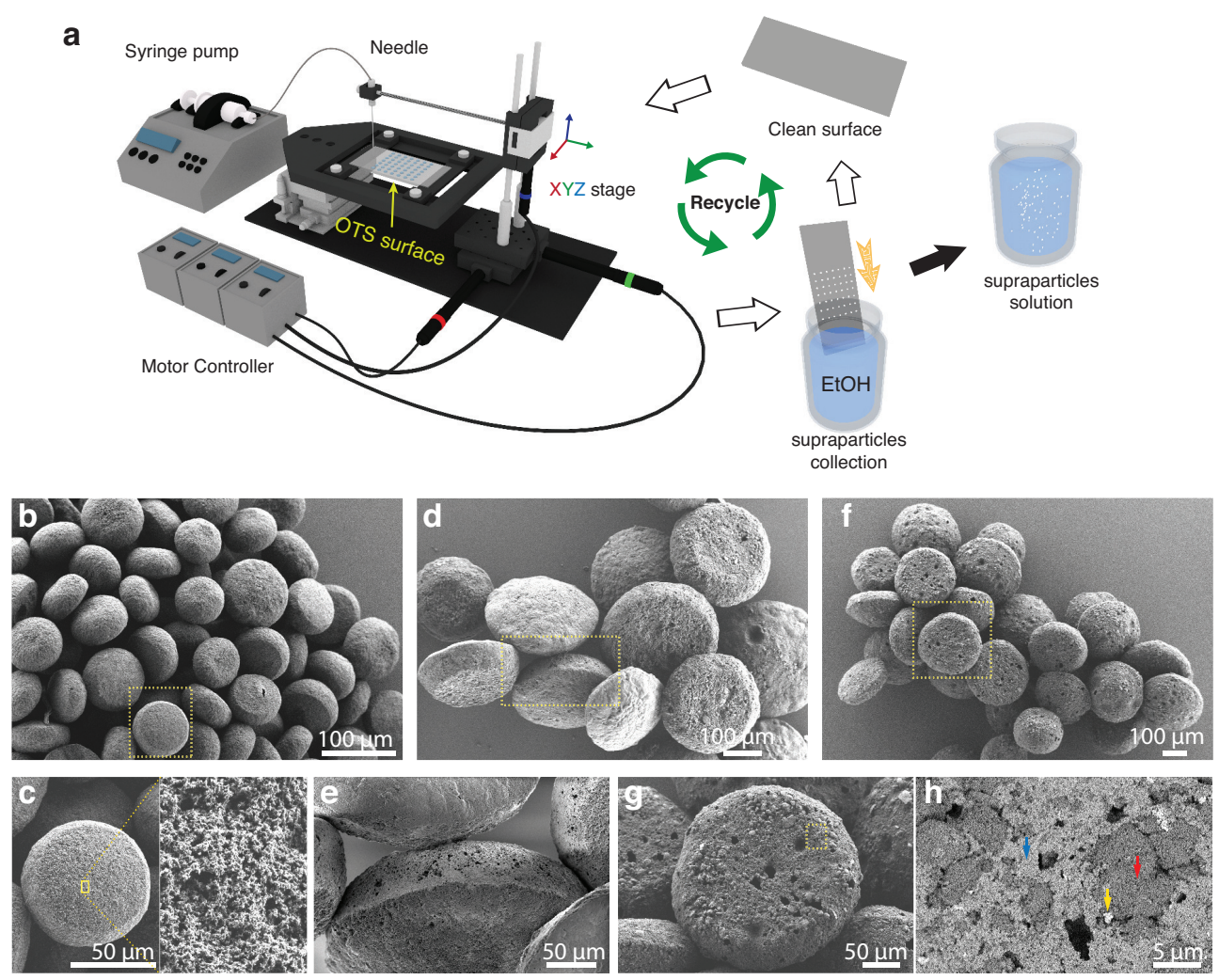

Figure 6.4: Scalability of the process with different and multiple types of nanoparticles. a, Demonstration of the flexible and handy scalability of supraparticle fabrication on OTMS/OTS surface. As self-lubrication and the robust surfaces allow for a simple harvesting process and to recycle the surfaces. b-h are SEM images of the generated supraparticles. b, Large quantities of generated porous $\mathrm{TiO}_{2}$ supraparticles. Panel (c) is the zoom into the porous surface of the particle. d, Bunches of porous supraparticles made by $\mathrm{TiO}_{2}(0.05 \mathrm{vol} \%)$ and $\mathrm{SiO}_{2}(0.05$ vol\%) nanoparticles. Panel $(\mathbf{e})$ is a zoom into the side of the particles. $\mathbf{f}$, Bunches of porous supraparticles with three different nanoparticles, $\mathrm{TiO}_{2}$ (0.06 vol\%), $\mathrm{SiO}_{2}$ (0.03 vol\%), and $\mathrm{Fe}_{3} \mathrm{O}_{4}(0.01$ vol\%). Panels $\mathbf{g}$ and $\mathbf{h}$ are a sequence of two zooms into the panel $\mathbf{f}$. In panel $\mathbf{h}$, the supraparticle surface was imaged with energy-selective backscatter (EsB) detector to present different materials in different grey levels: $\mathrm{Fe}_{3} \mathrm{O}_{4}$ (bright spots pointed by the yellow arrow), $\mathrm{TiO}_{2}$ (light grey regions by the blue arrow), $\mathrm{SiO}_{2}$ (dark grey regions by the red arrow). Darkness indicates holes.

particles by the self-assembly of $\mathrm{TiO}_{2}$ nanoparticles (Fig.6.4 $), \mathrm{TiO}_{2} \& \mathrm{SiO}_{2}$ nanoparticles (Fig. 6.4 d), and $\mathrm{TiO}_{2} \& \mathrm{SiO}_{2} \& \mathrm{Fe}_{3} \mathrm{O}_{4}$ nanoparticles (Fig. 6.4 ). Table 6.1 in the Supplementary Material lists the composition of the ouzo solutions. Figure 6.4 displays the porous surface of the $\mathrm{TiO}_{2}$ supraparticles. For the $\mathrm{TiO}_{2} \& \mathrm{SiO}_{2}$ supraparticles, different roughnesses are observed at the top surface and the bottom surface (Fig.6.4 ). The calculated porosity is 
Table 6.1: Composition list of the colloidal solutions for Figure 6.4

\begin{tabular}{|c|c|c|c|c|c|c|}
\hline & \multicolumn{3}{|c|}{ Nanoparticles } & \multicolumn{3}{|c|}{ Ouzo solution } \\
\hline & $\mathrm{TiO}_{2}$ & $\mathrm{SiO}_{2}$ & $\mathrm{Fe}_{3} \mathrm{O}_{4}$ & Oil & Ethanol & Water \\
\hline Fig. 6.4b & $0.005 \mathrm{v} \%$ & - & - & $1.2 \mathrm{v} \%^{\mathrm{a}}$ & $58.8 \mathrm{v} \%$ & $\sim 40 \mathrm{v} \%$ \\
\hline Fig. $\overline{6.4} \mathrm{~d}$ & $0.05 \mathrm{v} \%$ & $0.05 \mathrm{v} \%$ & - & $1.8 \mathrm{v} \%^{\mathrm{b}}$ & $58.2 \mathrm{v} \%$ & $\sim 40 \mathrm{v} \%$ \\
\hline Fig. $\overline{\overline{6.4}} f$ & $0.06 \mathrm{v} \%$ & $0.03 \mathrm{v} \%$ & $0.01 \mathrm{v} \%$ & $1.7 \mathrm{v} \%^{\mathrm{b}}$ & $58.3 \mathrm{v} \%$ & $\sim 40 \mathrm{v} \%$ \\
\hline
\end{tabular}

${ }^{a}$ Anise oil (Sigma-Aldrich; Anise oil);

b Trans-anethole oil (Sigma-Aldrich; trans-anethole, $\geq 99.8 \%$ ).

around $93 \%$. Figures $6.4 \mathrm{gh}$ are a sequence of zooms into the surface of the $\mathrm{TiO}_{2} \& \mathrm{SiO}_{2} \& \mathrm{Fe}_{3} \mathrm{O}_{4}$ supraparticle. The calculated porosity is around $91 \%$. In Figure 6.4h, different materials are distinguishable on the surface through energy-selective backscatter detector (EsB): The bright spots pointed at by the yellow arrow are $\mathrm{Fe}_{3} \mathrm{O}_{4}$ nanoparticles; the light grey regions (the blue arrow) are $\mathrm{TiO}_{2}$ nanoparticles; the dark grey regions (the red arrow) are $\mathrm{SiO}_{2}$ nanoparticles. The darkness indicates holes on the surface.

\subsection{Conclusion and outlook}

In conclusion, our new method of mass production of self-lubricating, selfassembled supraparticles is a dramatic improvement over evaporation-driven supraparticle self-assembly on super liquid-repellent surfaces and lubricantimpregnate surfaces. With our technique, commonly used hydrophobic surfaces are sufficient for the supraparticle fabrication, which improves flexibility, operability, and cost-efficiency of the fabrication. Moreover, the shape of the generated supraparticle is tunable by varying the oil-to-nanoparticle ratio of colloidal solutions. Combing our method with piezoacoustic inkjet technology can dramatically scale up fabrication, as there is no limitation on supraparticle collection. The generated highly porous supraparticles with multi-scale, fractal-like inner structures are suitable for many practical applications, such as catalysis, photonics, chromatography, environmental pollution management, and material science [163, 167-[171, 173, 174, 176, 177, 192]. The self-lubrication effect, in conjunction with easy detachments of the threedimensional particle aggregates after the particle-laden drop evaporation, implies potential applications in surface self-cleaning as well. We also note that the nucleated oil microdroplets in the colloidal drop can act as a carrier phase for different purposes. Additionally, by controlling the composition and temperature of the ouzo solution, different morphological features of the nucleated 
oil microdroplets - size, number, distribution - are tuneable [193]. Therefore, we expect more complex and exciting supraparticles created through this route.

\subsection{Method details and supplementary information}

\subsubsection{Preparation of colloidal ouzo solution}

The purchased nanoparticles, titanium (IV) oxide (Aldrich, nanopowder $21 \mathrm{~nm}$, $\geq 99.5 \%$ ), silicon dioxide (Aldrich, nanopowder $10-20 \mathrm{~nm}, \geq 99.5 \%$ ), iron (II,III) oxide (Aldrich, nanopowder $50-100 \mathrm{~nm}, 97 \%$ ) were burned at $400{ }^{\circ} \mathrm{C}$ for one hour to remove surfactants or contaminants attached on the particles before use. After that, the cleaned particles were added to specific amounts of Milli-Q water (produced by a Reference A+ system (Merck Millipore) at $18.2 \mathrm{M} \Omega \cdot \mathrm{cm}$ at $25^{\circ} \mathrm{C}$ ) to make nanoparticle suspensions. Trans-anethole oil (Aldrich, 99\%) and ethanol (Boom BV, $100 \%$ (v/v), technical grade) were used as received. Ethanol-oil (anethole) solutions were prepared separately beforehand and then mixed with the nanoparticle suspensions to make the final colloidal ouzo solutions with the required compositions for the experiments. We performed each mixing step in an ultrasonic bath for around $20 \mathrm{~min}$.

\subsubsection{Preparation of hydrophobic substrates}

The chemicals used for the hydrophobic substrate preparation, trimethoxy(octadecyl)silane (Aldrich, 90\%), toluene (Aldrich, 99.8\%), tetrahydrofuran (Aldrich, $\geq 99.9 \%$ ), and ethanol (Boom BV, $100 \%$ (v/v), technical grade) were used as received as well. In our experiments, the microscope glass slides (Thermo Scientific) were used as solid substrates for the OTMS layer coating. We first carefully wiped the glass slides with ethanol wetted tissue for mechanically removing contaminants from the surfaces. Then the slides were successively sonicated in fresh acetone, ethanol, and Milli-Q water, each for $15 \mathrm{~min}$, to remove organic contaminants from the surfaces. We repeated this step once and dried the slides by nitrogen flow. Then the slides were cleaned by plasma cleaner for $10 \mathrm{~min}$. After that, the cleaned glass slides were immersed into the coating mixture of $1 \mathrm{vol} \%$ octadecyltrimethoxysilane and $99 \mathrm{vol} \%$ toluene for $3 \mathrm{~h}$. After that, the coated slides were removed and then put into fresh toluene and tetrahydrofuran successively to dissolve the unlinked octadecyltrimethoxysilane above the surfaces. Finally, we dried the slides by nitrogen flow and put them in a clean petri dish for temporary storage. The preparation of octadecylsilanes(OTS)-treated substrate follows the same process. 


\subsubsection{Imaging analyses}

We used self-developed Matlab scripts to extract the profiles of the generated supraparticles in the side-view pictures. The details of the method refer to our previous work [101]. Through the top-view images, we confirmed the analyzed supraparticles are axisymmetric.

\subsubsection{Initial oil-to-nanoparticle ratio to have spherical supraparticles}

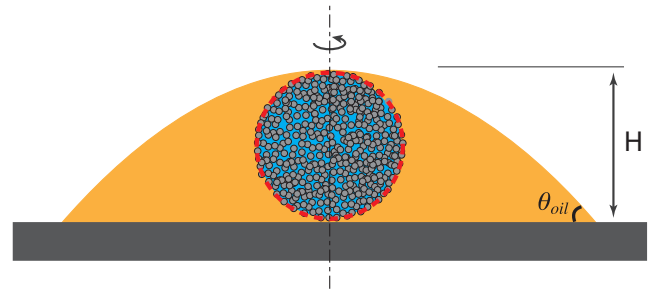

Figure 6.5: Schematic illustration of the model

Illustration Figure 6.5 shows a spherical supraparticle (SP) submerged in a spherical-cap oil drop. The maximum size of the supraparticle is the height $H$ of the oil drop. From simple geometrical consideration, we obtain the volume of the spherical supraparticle

$$
V_{\mathrm{SP}}=\frac{1}{6} \pi H^{3}
$$

and the volume of the oil droplet

$$
V_{\mathrm{OD}}=\frac{1}{6} \pi H^{3}\left[1+3 \cot ^{2}\left(\frac{\theta_{\mathrm{oil}}}{2}\right)\right],
$$

with the oil contact angle $\theta_{\text {oil }}$. The volume of the oil is estimated as

$$
V_{\mathrm{oil}}=V_{\mathrm{OD}}-V_{\mathrm{SP}}
$$

while the total volume of nanoparticles is given by

$$
V_{\mathrm{NP}}=V_{\mathrm{SP}}(1-\phi),
$$

where $\phi$ is the porosity of the supraparticle. Thus, we obtain an estimate of the initial oil-to-nanoparticles ratio $k^{*}$ to have spherical supraparticles, namely

$$
k^{*}=\frac{3}{1-\phi} \cot ^{2} \frac{\theta_{\mathrm{oil}}}{2},
$$

which only depends on the oil contact angle $\theta_{\text {oil }}$ and the supraparticle porosity $\phi$ and is independent of the drop size. In equation 6.3 , we use $V_{\mathrm{SP}}$ instead of $V_{\mathrm{NP}}$, because we assume that the residual water fills the porous structure. 


\section{Part III}

\section{Surface nanodroplets}





\section{7}

\section{Surface nanodroplets formation}

Solvent exchange is a generally-used approach for producing many nanoscale droplets on an immersed substrate. In this process, a good solvent is displaced by a poor solvent of oil, leading to oil nanodroplets nucleation and subsequent growth on the substrate. This work is the first attempt to quantitatively understand the relationship between the droplet size and the flow conditions during the solvent exchange, and to pave the way for the droplet size control. The experimental results show that the droplet volume increases with increasing Peclet number of the flow as $\propto P e^{3 / 4}$, in good agreement with our theoretical analysis. We also reveal that the buoyancy effects contribute to the formation of bigger and less homogeneously distributed droplets in tall channels.

\subsection{Introduction}

Nanodroplets on a solid surface (i.e. surface nanodroplets) have practical implications for high-throughput chemical and biological analysis, lubrications, lab-on-chip devices, and near-field imaging techniques. Oil nanodroplets can be produced on a solid-liquid interface in a simple step of solvent exchange in which a good solvent of oil is displaced by a poor solvent. In this work, we

*Based on: X. Zhang, Z. Lu, H. Tan, L. Bao, Y. He, C. Chao, D. Lohse, Formation of surface nanodroplets under controlled flow conditions, Proceedings of the National Academy of Sciences 112, 9253-9257 (2015).

${ }^{\dagger}$ Imaging analysis, convection effects analysis (in part), data processing (in part) and figure preparation by $\mathrm{H}$. Tan. 
experimentally and theoretically investigate the formation of nanodroplets by the solvent exchange process under well-controlled flow conditions. We find that the contact angle of the nanodroplets is independent of the flow condition. However, there are significant effects from the flow rate and the flow geometry on the droplet size. We develop a theoretical framework to account for these effects. The main idea is that the droplet nuclei are exposed to an oil oversaturation pulse during the exchange process. The analysis gives that the volume of the nanodroplets increases with the Peclet number $\mathrm{Pe}$ of the flow as $\propto P e^{3 / 4}$, which is in good agreement with our experimental results. In addition, at fixed flow rate and thus fixed Peclet number, larger and less homogeneously distributed droplets formed at less narrow channels, due to convection effects originating from the density difference between the two solutions of the solvent exchange. The understanding from this work provides valuable guidelines for producing surface nanodroplets with desired sizes by controlling the flow conditions.

Nanoscale droplets on a substrate are an essential element for a wide range of applications, namely lab-on-chip devices, simple and highly-efficient miniaturised reactors for concentrating products, high-throughput single-bacteria or single-biomolecular analysis, and high-resolution imaging techniques, amongst others [194-197]. Quite some effort has been devoted to produce a large amount of nanodroplets in a controlled way. The current techniques include trapping by microcavities, emulsion direct adsorption, microprinting and others [198. The solvent exchange process is a simple and generic approach for producing droplets or bubbles at solid-liquid interfaces that are only several tens to hundreds nanometers in height, or a few femtoliter in volume [63, 173, 199 201]. It has attractive advantages, such as its capability of producing a large number of nanodroplets in one simple step, and its generality in chemical composition of the droplet liquid, and flexibility in aspect ratio of the droplets and spatial structure or size of the substrate [73, 202].

For the formation of surface nanodroplets by solvent exchange, a hydrophobic substrate is exposed sequentially to two miscible solutions of oil, where the second solvent has a lower solubility of oil than the first. Such solubility difference leads to supersaturation of the liquid with oil during the solvent exchange and consequently to the nucleation of nanodroplets on the substrate. The analogue technique in a bulk system is called solvent shifting or nanoprecipitaion through the Ouzo effect [41, which has been increasingly applied to obtain nanodroplets in a surfactant-free emulsion [203], monodispersed polymeric nanoparticles with precisely-controlled sizes [202, 204 206], or assemble colloidal particles on a microscale [207.

Although the chemical composition of the solutions has been used to adjust 
A
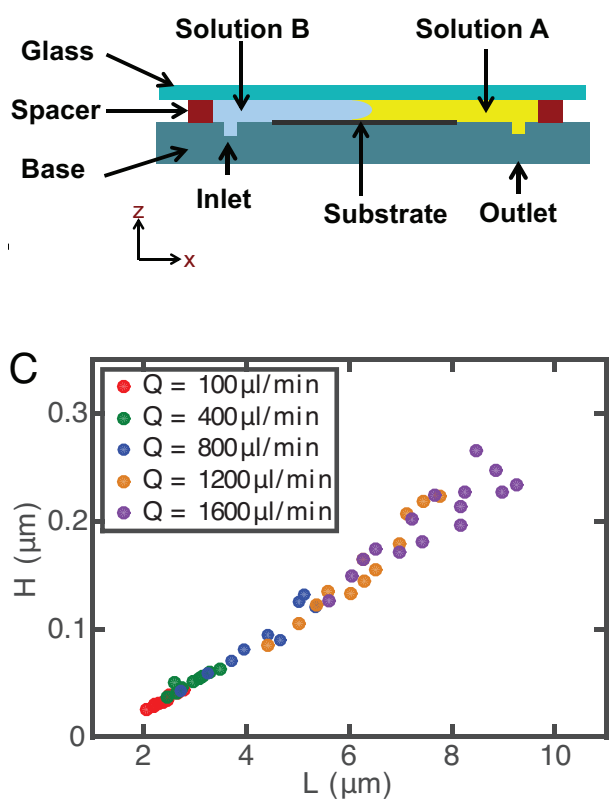

B
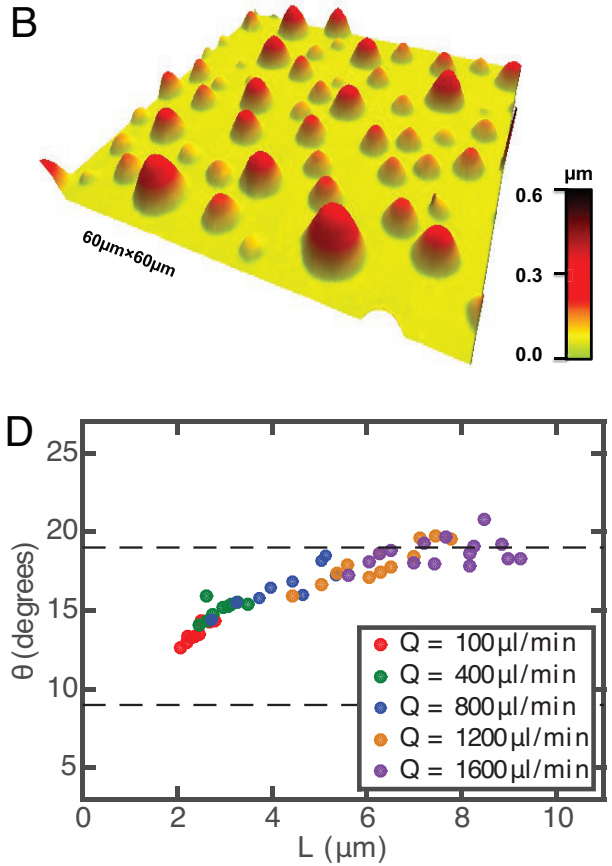

Figure 7.1: Solvent exchange process and morphologic features of surface droplets formed at different flow rates for the narrow channel $(h=0.33 \mathrm{~mm})$. (a) Schematic drawings of a fluid channel. The channel consists of a glass top window, spacer and a base. The hydrophobic substrate is placed inside the cell, facing to the transparent glass window. The distance between the substrate and the glass bottom surface can be adjusted by the thickness of the spacer. The flow direction is in $\mathrm{x}$ direction. (b) shows a representative AFM image of the polymerized droplets. (c) Droplet height, $\mathrm{H}$, and (d) the contact angle, $\theta$, versus the lateral diameter, $\mathrm{L}$, of the nanodroplets. The macroscopic advancing and receding angles are labelled for comparison.

the average size of the droplets [202], the flow properties dramatically complicate the formation of nanodroplets by the solvent exchange. The reason is that the mixing of the two liquids strongly depends on the flow conditions [208, 209]. Inspired by the pattern formation of mineral aggregates from liquid displacement under a quasi-2D flow conditions [210, 211, we may be able to control the droplet nucleation and growth by the flow conditions in a well-defined flow system. In this work, we theoretically and experimentally investigate the effects of the flow conditions on the formation of surface nanodroplets. We find that the averaged volume of surface nanodroplets increases with the Peclet number as $\propto P e^{3 / 4}$, in good agreement with the experiments. As far as we know, this work is the first attempt to quantitatively understand the effects of the flow conditions during the solvent exchange on the formation of surface nanodroplets. 


\subsection{Results and discussion}

\subsubsection{Droplet volume dependence on the flow rate}

The geometry of the solvent exchange channel and process is shown in schematic drawings in Figure 7.1(a). Three fluid channels with different heights were used and their dimensions are listed in Table 7.1. During the solvent exchange process, solution A (50\% ethanol aqueous solution saturated with polymerisable oil, with high oil solubility) was displaced by solution B (oil-saturated water, with low oil solubility). The injection of solution B was performed at a constant flow rate $Q$ controlled by a syringe pump. Once the solvent exchange was completed, the nanodroplets on the substrate were cured by photopolymerization.

Figure 7.1(b) show a representative AFM image of the polymerised nanodroplets. The polymerised droplets are spherical caps with a certain size distribution. The plot in Figure 7.1(c) shows that the droplet height increases monotonically from $10 \mathrm{~nm}$ to $300 \mathrm{~nm}$ as the lateral size increases from $2 \mu \mathrm{m}$ up to $10 \mu \mathrm{m}$, in consistency with the previous reports [73]. The contact angles of those polymerised droplets lie mainly between the macroscopic receding $\left(8^{\circ}\right)$ and advancing $\left(19^{\circ}\right)$ contact angles of the system Clearly, the flow rate does not influence the contact angle of the surface nanodroplets.

The droplet volume is calculated from its lateral diameter in the optical images of the polymerised droplets in Figure 7.2 , and the corresponding contact angle in Figure 7.1. Figure 7.2(d) shows the probability distribution function (PDF) of the droplet volume at different flow rates in the narrowest channel, where the distribution of the droplet volume became wider at a faster flow rate. To further examine effects of the flow rate on the droplet size, we measured the lateral diameter of droplets that were produced from different flow rates at all three channels. The averaged lateral diameter of the droplets versus the flow rate is plotted in Figure 7.2 (e), which shows a fast increase of the droplet size with an increase in the flow rate for all three channel heights. For instance, in the narrow channel the spatially averaged droplet diameter increased from $2 \mu \mathrm{m}$ to $10 \mu \mathrm{m}$ (20 nm to $300 \mathrm{~nm}$ in height) as the flow rate increased from $100 \mu \mathrm{l} / \mathrm{min}$ to $2400 \mu \mathrm{l} / \mathrm{min}$. It shows the same trend for the two less narrow channels, but the absolute values are larger on average.

We analysed all droplet volumes over a surface area of $0.35 \mathrm{~mm}^{2}$ and obtained the averaged droplet volume per unit area of $\mu \mathrm{m}^{2}$. The plot in Figure 7.2 (f) shows a sharp increase of the averaged droplet volume with increasing flow rate. The same data are shown in Figure 7.2 (g) (in a log-log plot)

\footnotetext{
${ }^{\ddagger}$ Strictly speaking, the contact angle of a liquid droplet before the polymerisation is about $1-2^{o}$ larger than that of its polymerised counterpart due to curing shrinkage.
} 


\section{A $\quad \mathrm{h}=0.33 \mathrm{~mm}$}

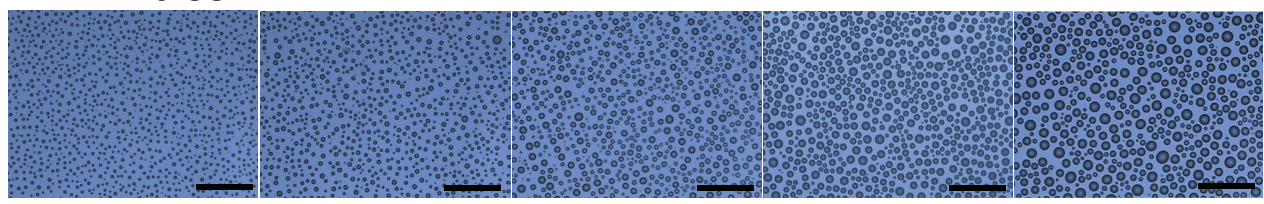

B $\quad \mathrm{h}=0.68 \mathrm{~mm}$

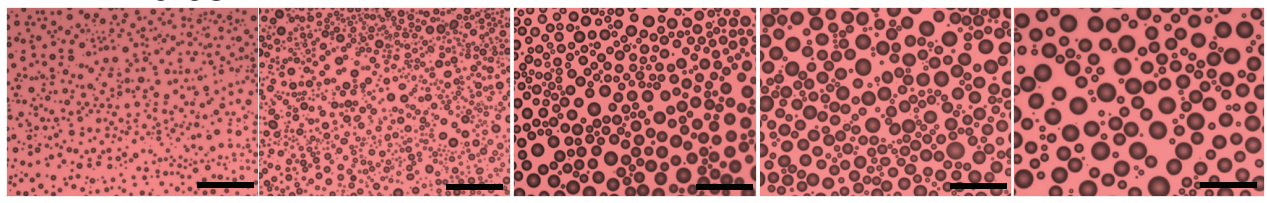

\section{C $\quad \mathrm{h}=2.21 \mathrm{~mm}$}
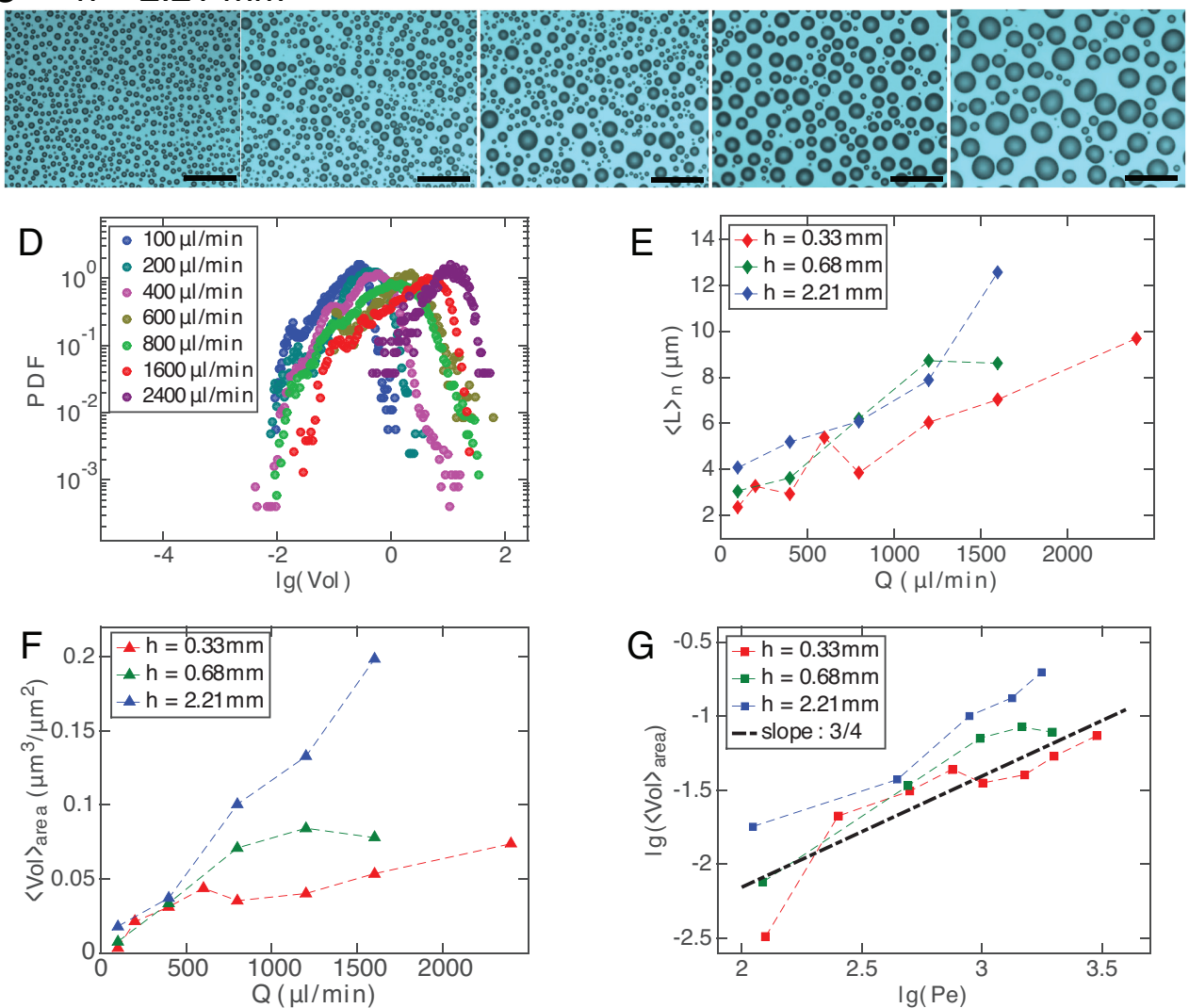

Figure 7.2: Optical images and size of surface droplets formed at different flow rates in three channels. (a-c) shows reflection-mode optical images of the polymerised droplets at different flow rates. Length of scale bars: $50 \mu \mathrm{m}$. The flow rates were $100 \mu \mathrm{l} / \mathrm{min}, 400 \mu \mathrm{l} / \mathrm{min}, 800$ $\mu \mathrm{l} / \mathrm{min}, 1200 \mu \mathrm{l} / \mathrm{min}$, and $1600 \mu \mathrm{l} / \mathrm{min}$. The plots show the PDF of the droplet volume produced in the narrowest channel $(d)$, the averaged lateral diameter $(e)$ and the averaged volume $(f)$ of the surface droplets at different flow rates. $(g)$ shows the averaged volume of droplets per $\mu \mathrm{m}^{2}$ as function of the Peclet number on a log-log plot. The dashed line shows the scaling law $\langle\mathrm{Vol}\rangle_{\text {area }} \propto P e^{3 / 4}$. 
versus the Peclet number

$$
P e=\frac{\bar{U} h}{D}=\frac{Q}{w D}
$$

of the flow, where $D$ is the diffusion constant. The data can be described with the scaling law $\propto P e^{3 / 4}$. Later we will show that this scaling law between the droplet volume and the $P e$ number is in a good agreement with the theoretical prediction.

A

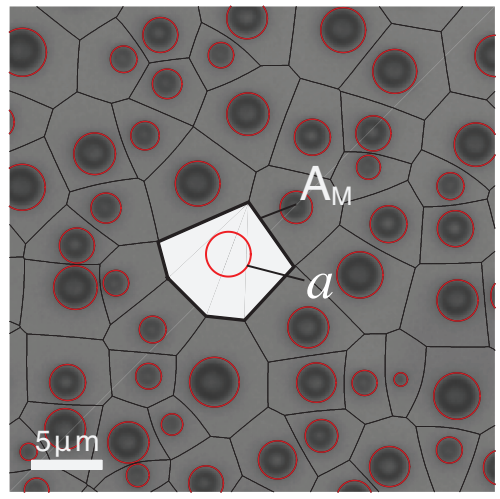

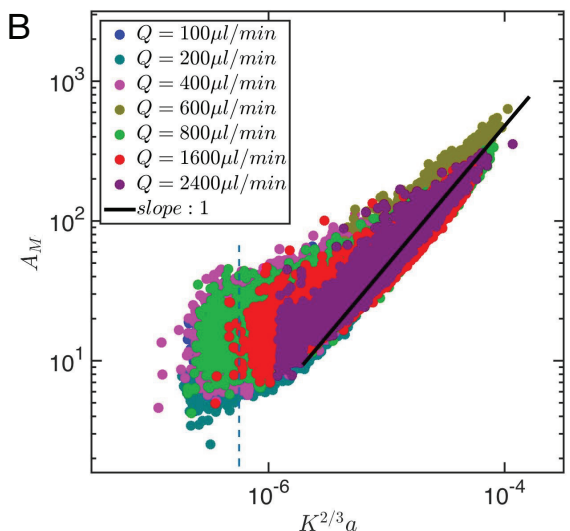

Figure 7.3: (a) Modified Voronoi tessellation of a typical image of the droplets. Red circles indicate the detected footprints of droplets, and black lines are the edges of Modified Voronoi cells. (b) The correlation between the footprint areas $a$ and the Modified Voronoi cell areas $A_{M}$

It is crucial to identify the formation mechanism of the droplets before we develop a theoretical model to understand the effect of the flow rate. In our tertiary system of ethanol-oil-water, surface nanodroplets may nucleate on the surface, or through the standard "ouzo effect", namely nucleate in the bulk liquid [41, 206, 212 214, and only later adsorb onto the surface. We analysed the droplet volume and the surrounding area of droplet-depleted zone, following the modified Voronoï tessellation method in our previous work [215]. In Figure 7.3, we show the relation between the depleted area and the area of the corresponding footprint of nanodroplets produced at different flow rates. For the relatively large nanodroplets, the depleted area is proportional to the droplet footprint area. This correlation clearly suggests that surface nanodroplets are not from random adsorption of emulsion droplets, but from heterogenous nucleation and subsequent diffusion-driven growth. In this process, the oil dissolved in the bulk is consumed by the growth of the droplets, leading to the droplet-depleted area in the surrounding region. The same diffusion-driven mechanism and the same correlation between the depleted area and the footprint area was also observed (and derived) for the spatial 

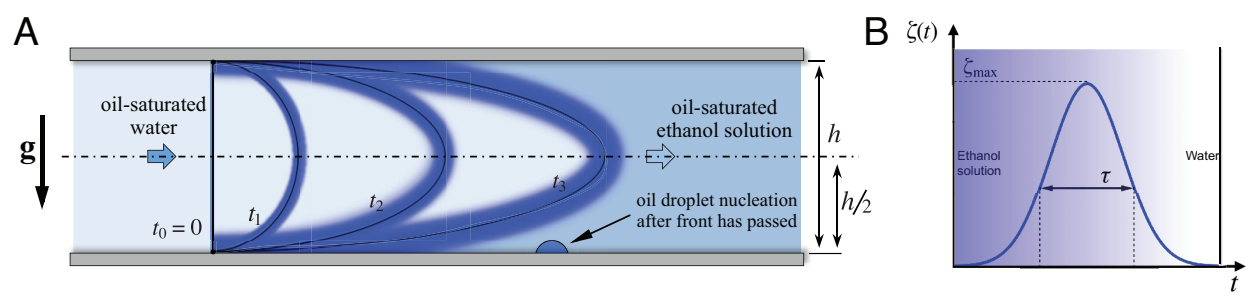

Figure 7.4: (a) Parabolic flow profiles for various times $t \geq 0$. Note the no-slip boundary condition for the flow. The originally sharp interface will broaden with time. (b) Approximate temporal evolution of the oversaturation $\zeta(t)$ at fixed position downstream. The width $\tau$ of the pulse is defined through $\int_{-\infty}^{\infty} \zeta(t) d t=\zeta_{\max } \tau$.

arrangement of surface nanobubbles formed by the solvent exchange method [215].

\subsection{A simple theoretical model}

\section{Mathematical description of the solvent exchange process}

As shown in the schematic drawings of Figure 7.1, the flow cell consists of a channel with height $h$ and channel width $w$. The maximal flow velocity is $U$, and mean flow velocity $\bar{U}$. The resulting flow rate is $Q=h w \bar{U}$, its Reynolds number $R e=\bar{U} h / \nu=Q /(w \nu)$. For the theory we will focus on laminar flow, i.e. $R e \lesssim 1$, which, as seen from Table 7.1 is justified for all channels. Furthermore, we will neglect the density contrast between the ethanol solution and the water (factor about 0.9), which, as we will see below, strictly speaking is only justified for the narrow channel case $h=0.33 \mathrm{~mm}$.

In principle, the solvent exchange process is described by the advection diffusion equations for the ethanol solution and water and the oil dissolved in it, with the no-slip boundary conditions on all walls. The initial conditions are such that in the left part of the channel there is no ethanol dissolved in the water, but oil up to its saturation concentration $c_{s, \text { wat }}$ in water. In the right part of the channel we have ethanol solution with dissolved oil at saturation concentration $c_{s, \text { eth }}>c_{s, w a t}$. At time $t=0$ the interface between the two parts of the flow is assumed to be sharp. From $t=0$ on, the flow is driven by a pump such that the flow rate $Q$ is constant. The interface will then develop a parabolic shape, corresponding to the laminar flow situation. It will smoothen out with advancing time, see the sketch Figure 7.4(a). Note that downstream in the region which was initially filled with ethanol solution the front will hit the surface nearly parallel to the surface, due to the no-slip boundary condition. The width of the front is given by the diffusion process of oil (and ethanol) towards the water and water towards the ethanol. The 
boundary conditions for the oil are no flux boundary conditions at the top and bottom wall. Once there is oil droplet nucleation, the oil concentration equals $c_{s, w a t}$ at the droplet-water interface.

For nucleating and growing oil droplets the most relevant quantity is the oil oversaturation

$$
\zeta(t)=\frac{c_{\infty}(t)}{c_{s}}-1 .
$$

As the liquid is saturated in the ethanol phase, there we have $c_{\infty}=c_{s, \text { eth }}$ and thus $\zeta=0$. The same holds in the water oil phase, $c_{\infty}=c_{s, w a t}$ and thus also $\zeta=0$. However, in the broadening front around the interface we have oversaturation $\zeta>0$, as oil diffuses from the ethanol-rich phase towards the water-rich phase, in which it is less soluble. The maximum oversaturation is

$$
\zeta_{\text {max }}=\frac{c_{s, e t h}}{c_{s, w a t}}-1>0 .
$$

At fixed position downstream (what initially is the ethanol phase) we first have no oversaturation, $\zeta=0$. Then the front is passing by during which $\zeta(t)>0$ so that oil droplets can nucleate and grow. In the end the ethanol is basically fully replaced by the oil-saturated water and then again $\zeta=0$. This front is characterised by the maximum $\zeta_{\max }$ and by some temporal width $\tau$, see Figure 7.4(b). One may be tempted to argue that this temporal width $\tau$ depends on the flow velocity. However, note that independent of the flow velocity the nucleating droplets on the surface are horizontally hit by the mixing front, given a no-slip boundary condition. We therefore argue that in our laminar flow cell $\tau$ is independent of $U$ and purely given by the diffusion process, suggesting $\tau \sim \frac{h^{2}}{D}$. For $h=0.33 \mathrm{~mm}$ as in the narrow channel experiments and $D=1.6 \times 10^{-9} \mathrm{~m}^{2} / \mathrm{s}$ we get $\tau \approx 60 \mathrm{~s}$.

\section{Growth of a nucleated droplet}

Once the oversaturation front passes, droplets nucleate and grow. Here we focus on an individual droplet. We assume that there is no pinning and that the contact angle $\theta$ is thus constant. The size of the droplet is characterised by its lateral extension $L$. Alternatively, we can use the radius of curvature $R$ of the oil-water interface as a characterisation of the droplet size, which we will do here. Then $\operatorname{Vol} \sim R^{3}$ for the droplet volume, as $\theta$ is constant. Assuming different growth modes as stick-slip or stick-jump [36] would only change prefactors, but not the essence of below derivation.

Strictly speaking, for the droplet growth no symmetry holds: The axial symmetry, which is obeyed for a drop diffusively growing in still liquid, is broken by the flow direction. Nonetheless, to obtain the scaling relations we 
can still assume the diffusive growth equation even in spherical symmetry,

$$
\dot{m}=4 \pi \rho_{\text {oil }} R^{2} \dot{R}=\left.4 \pi D R^{2} \partial_{r} c\right|_{R} .
$$

In this laminar flow situation the concentration gradient at the interface $\left.\partial_{r} c\right|_{R}$ is given by the oil concentration difference between the oil concentration in the flow $c_{\infty}$ and at the interface $c_{s, w a t}$ and by the thickness $\lambda$ of the concentration boundary layer, for which we assume a Prandtl-Blasius Pohlhausen's type behavior [216, 217] as appropriate for laminar flow. Then $\lambda \sim \frac{R}{\sqrt{P e}}$. I.e., we have

$$
\left.\partial_{r} c\right|_{R} \sim \frac{c_{\infty}(t)-c_{s, w a t}}{\lambda} \sim c_{s, w a t} \frac{\zeta(t)}{\lambda} \sim c_{s, w a t} \sqrt{P e} R^{-1} \zeta(t) .
$$

Plugging this into equation (7.4) we obtain a simple ODE for $R(t)$, namely

$$
R \dot{R} \sim \frac{D c_{s, w a t}}{\rho_{\text {oil }}} \sqrt{P e} \zeta(t),
$$

which can easily be integrated respectively from 0 to the final radius $R_{f}$ or from 0 to $t=\infty$, giving

$$
R_{f} \sim\left(\frac{D c_{s, w a t}}{\rho_{\text {oil }}} \zeta_{\text {max }} \tau P e^{1 / 2}\right)^{1 / 2} .
$$

Here we have used $\int_{0}^{\infty} \zeta(t) d t=\zeta_{\max } \tau$. Using our prior assumptions $\tau \sim \frac{h^{2}}{D}$ (in particular that it is flow rate independent) and equation $(7.3)$ on $\zeta_{\max }$ we obtain

$$
V o l_{f} \sim R_{f}^{3} \sim h^{3}\left(\frac{c_{s, w a t}}{\rho_{\text {oil }}}\right)^{3 / 2}\left(\frac{c_{s, \text { eth }}}{c_{s, w a t}}-1\right)^{3 / 2} P e^{3 / 4}
$$

for the final volume of the droplet after the solvent exchange. The scaling $V_{o l} \sim \mathrm{Pe}^{3 / 4}$ of our theoretical model is in good agreement with the experimental data shown in Figure 7.2 (g), given that the droplet number density is flow rate independent.

\subsection{Buoyancy driven convection effects}

We now examine the effect of the channel height on the droplet formation. First of all we characterised the flow in the channels by using fluorescent microscopy, while water was dyed green to assist the visualisation. Top-view videos of the entire exchange process are provided in the Supporting Videos. The snapshots in Figure 7.5(a) show that in the narrowest channel the water displaced the ethanol solution in a smooth and continuous manner. The fluorescent intensity on a specific location increased with time smoothly, as the 


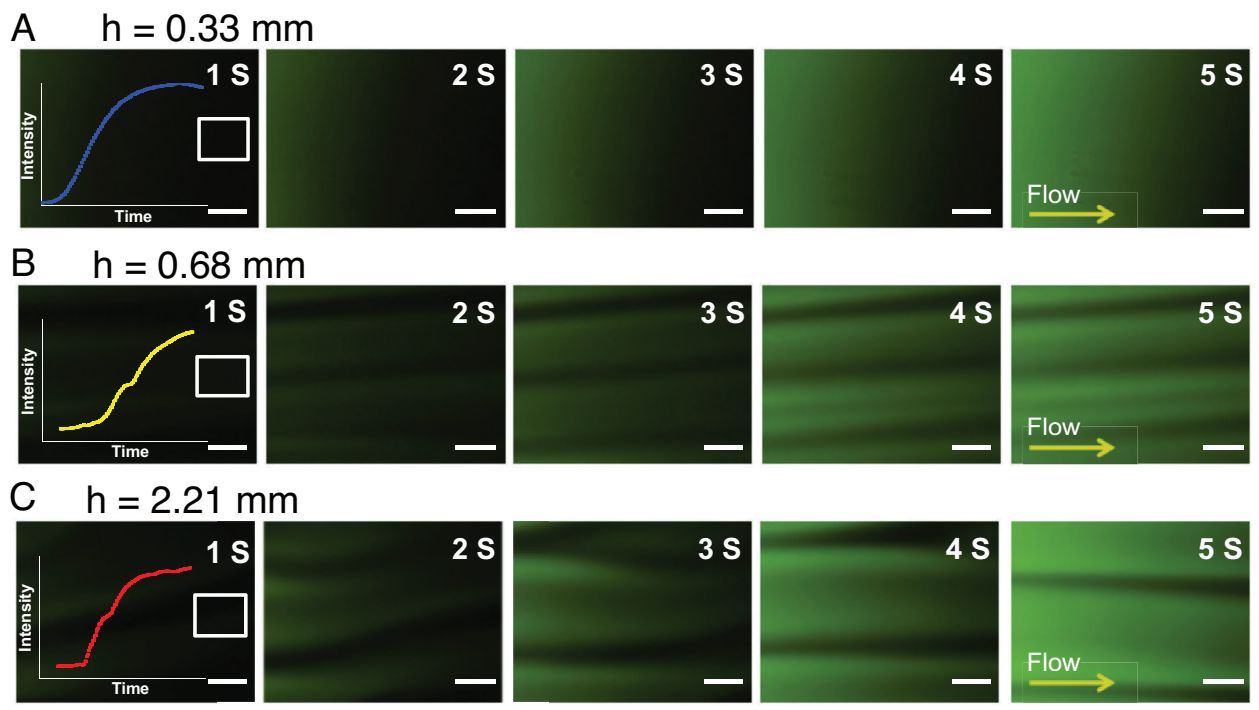

Figure 7.5: Top-view snapshots of the flow during the solvent exchange in three channel heights. The direction of the water (dyed green) in the fluorescent images was from left to right. Length of the scale bar: $200 \mu \mathrm{m}$. Insert is the curve of integrated optical density of selected area as function of time as water was pushed through the channel. The mean flow velocity $\bar{U}=0.36 \mathrm{~mm} / \mathrm{s}$ in all three channels.

concentration of water increased in the liquid phase. The top-view snapshots in Figure 7.5(b) and Figure 7.5(c) show the flow patterns for the two less narrow channels at the same mean flow velocity of $\bar{U}$ of $0.36 \mathrm{~mm} / \mathrm{s}$. Straight and regular fingers were clearly visible in the channel with $h=0.68 \mathrm{~mm}$, while the flow already developed whirling patterns for $h=2.21 \mathrm{~mm}$. The time evolution of the fluorescent intensity of the dye in water shows some deflections and jumps in the intensity, indicating the abrupt change in water contents due to the non-uniform mixing. Such flow features are in contrast to the smooth flow in the narrow channel at $h=0.33 \mathrm{~mm}$. Note that in all three cases the flow is still laminar, see Table 7.1 .

The reason for the different flow patterns in the less narrow channels is that for them we must consider the density difference between the two miscible liquids [218, 219]. The density of water is $1 \mathrm{~g} / \mathrm{ml}$ while the density of the ethanol aqueous solution is $\sim 0.90 \mathrm{~g} / \mathrm{ml}$. At solvent exchange, at the bottom side of the channel above the plate, the lighter ethanol will be pushed below the entraining heavier water, potentially leading to some buoyancy driven convection rolls. To estimate when these convection rolls set in, we can define a "Rayleigh number"

$$
R a=\frac{\Delta \rho g(h / 2)^{3}}{\mu D_{e, w}}
$$




\section{A}
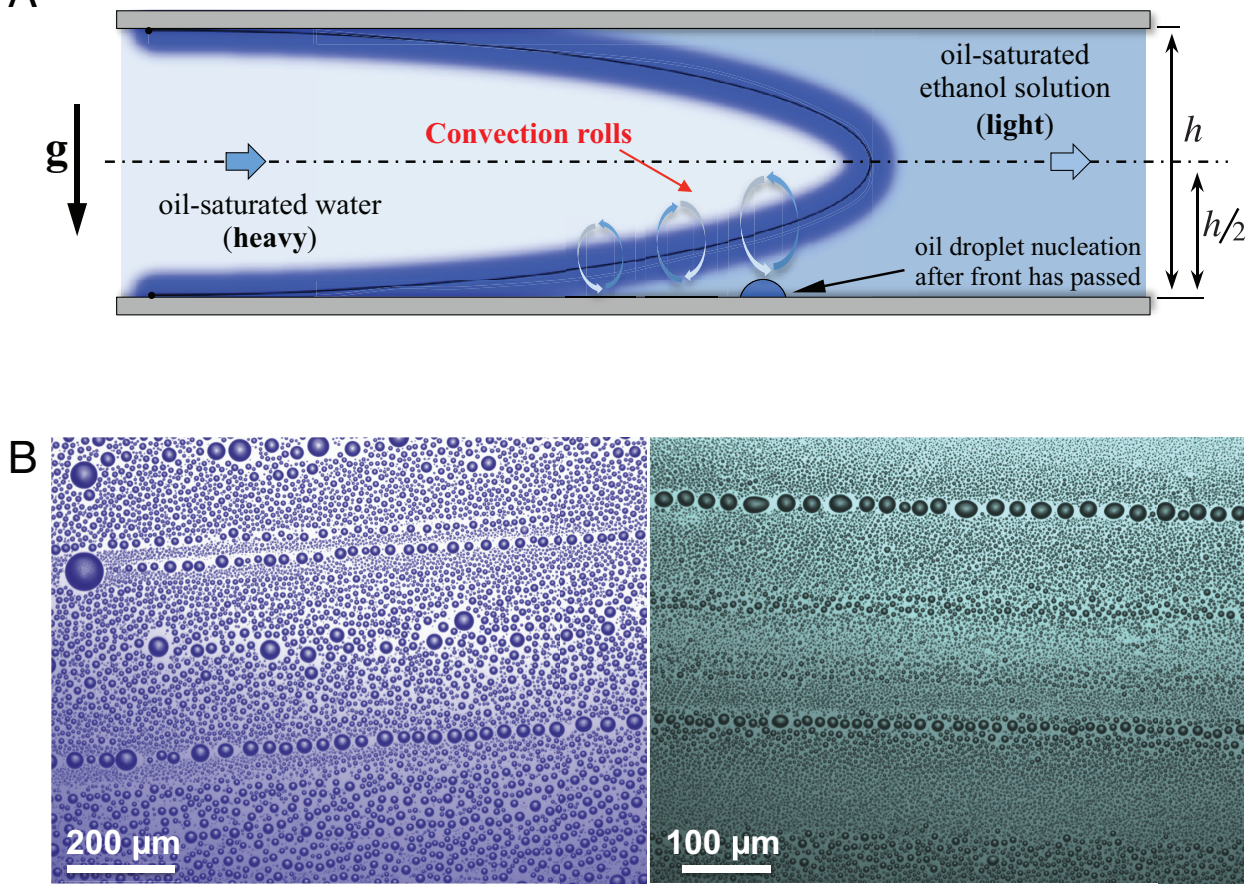

Figure 7.6: Solvent exchange and nanodroplet lines in the less narrow channels. (a) The illustration shows convection rolls in the bottom part of interface between two solutions during the solvent exchange, where the heavy oil-saturated water is above the light oilsaturated ethanol aqueous solution. (b) Two representative images of the nanodroplet lines formed in two less narrow channels. Left panel: $Q=1600 \mu \mathrm{l} / \mathrm{min}$ and $h=0.68 \mathrm{~mm}$. Right panel: $Q=400 \mu \mathrm{l} / \mathrm{min}$ and $h=2.21 \mathrm{~mm}$. The droplets organise in rows, reflecting the convection rolls with axes in flow direction. These rolls enhance mixing, leading to larger droplets where the rolls hit the substrate.

where the density difference $\Delta \rho$ is $0.1 \mathrm{~g} / \mathrm{ml}$, the gravitational acceleration $g$ is $9.8 \mathrm{~m} / \mathrm{s}^{2}, \mu$ is the dynamic viscosity of ethanol solution, and the mass diffusion coefficient of ethanol and water $D_{e, w}$ at $300 \mathrm{~K}$ is $1.6 \times 10^{-9} \mathrm{~m}^{2} / \mathrm{s}$. Convection only occurs at the lower half of the channel where heavy liquid (water) is above light liquid (ethanol), see Figure 7.6(a). Therefore we take $h / 2$ as vertical length-scale in equation (7.9). The resulting estimated Rayleigh numbers are $\approx 1.1 \times 10^{3}, 1.0 \times 10^{4}$, and $3.5 \times 10^{5}$ for the channel heights of $0.33 \mathrm{~mm}, 0.68$ $\mathrm{mm}$ and $2.21 \mathrm{~mm}$, respectively. So the convection rolls (in the top view seen as stripes) only occur in the two less narrow channels, where the Rayleigh number is larger than the critical Rayleigh number 1708 [220]. The existence of convection rolls for the two less narrow channels also explain why for fixed flow rate and thus fixed Peclet number, the average droplet size depends on the channel height, in particular for high flow rates, as seen in Figure 7.2(a)(b)(c). 
The convection rolls lead to a better mixing between ethanol and water, and thus better transport of oil towards the substrate and consequently to larger oil droplets.

The development of the secondary flow at a high flow rate may lead to some patterns of the droplets. Indeed, we observed that some of the droplets produced in the least narrow channel line up along the flow direction as shown in Figure 7.6(b). Such lines of droplets also formed at larger flow rate in the channel with $h=0.68 \mathrm{~mm}$. They form when the convection rolls hit the surface, bringing down oil rich liquid. All these features do not develop when we turn the channel by $90^{\circ}$ (see Supporting Figure 2), through which we eliminate buoyancy effects.

Table 7.1: Experimental cases and parameters for different fluid channel heights $h$. For all cases the width of fluid channels $w$ and the length $l$ are $14 \mathrm{~mm}$ and $56 \mathrm{~mm}$, respectively. $Q$ is the flow rate, $\bar{U}=Q /(w h)$ the mean flow velocity, $R e=\bar{U} h / \nu=Q /(w \nu)$ the Reynolds number, and $P e=\bar{U} h / D=Q /(w D)$ the Peclet number.

\begin{tabular}{cccccc}
$Q(\mu \mathrm{l} / \mathrm{min})$ & $R e$ & $P e$ & \multicolumn{3}{c}{$(\mathrm{mm} / \mathrm{s})$} \\
\hline 100 & 0.04 & 119 & 0.36 & 0.18 & 0.05 \\
200 & 0.09 & 238 & 0.72 & 0.35 & 0.11 \\
400 & 0.18 & 476 & 1.44 & 0.70 & 0.22 \\
600 & 0.27 & 714 & 2.16 & 1.05 & 0.32 \\
800 & 0.35 & 952 & 2.88 & 1.40 & 0.43 \\
1200 & 0.53 & 1429 & 4.32 & 2.10 & 0.65 \\
1600 & 0.71 & 1905 & 5.77 & 2.80 & 0.86 \\
2400 & 1.06 & 2857 & 8.66 & 4.20 & 1.29 \\
\hline
\end{tabular}

\subsection{Experimental method}

\subsubsection{Substrate and solutions}

A stock solution containing monomer and initiator was prepared by mixing 1, 6-hexanediol di-acrylate (HDODA) (80\%, Sigma-Aldrich) with 2-hydroxy2-methylpropiophenone ( $97 \%$, Sigma-Aldrich) in the ratio of 10:1. This solution of monomer precursors served as oil phase. $4 \mathrm{ml}$ of the monomer solution was added into $100 \mathrm{ml}$ ethanol/water (50 v\%: $50 \mathrm{v} \%)$ solution, and the bottom phase of the liquid was the solution A. Solution B was water saturated with HDODA. The hydrophobic substrate of OTS-Si was prepared and cleaned by following the protocol reported in previous work [73]. Before use, the OTS-Si was cleaned with chloroform, sonicated in ethanol, and dried 
with nitrogen. The advancing and receding contact angles of water were $112^{\circ}$ and $98^{\circ}$, respectively.

\subsubsection{Preparation, polymerisation and characterisation of nanodroplets}

The experimental setup is shown in the schematic drawing Figure 7.1(a). A flow cell was constructed by assembling a glass plate, a spacer and a base together, forming a channel where the OTS-Si was put in. The channel height between the OTS-Si substrate and glass plate was adjusted by the thickness of the spacer. $5 \mathrm{ml}$ solution A was first injected into the flow cell, followed by the injection of $10 \mathrm{ml}$ solution $\mathrm{B}$ with a constant flow rate controlled by a syringe pump. After the formation of the nanodroplets, the flow cell was illuminated under an UV lamp (20 W, $365 \mathrm{~nm}$ ) for $15 \mathrm{~min}$, allowing the polymerization of the monomer droplets. The substrate was then washed with ethanol and dried by a gentle stream of nitrogen. Images of the polymerised microdroplets were acquired using a reflection-mode optical microscopy. High resolution images of the polymerised microdroplets were also obtained from normal contact mode AFM imaging in air (Asylum Research, Santa Barbara, CA).

\subsubsection{Data analyses}

As shown in Figure $7.2 \mathrm{lbc}$, there are mass of surface nanodroplets in a wide range of sizes. To automatically extract the geometric information of these nanodroplets, we developed a MATLAB program, which integrate the circular Hough transform method [221] for the nanodroplet detection (red circles in Fig. 7.3 a). We assumed the nanodroplets have spherical-cap shapes, since the interfacial surface tension dominates the gravity here. Given the contact angle of the droplet liquid in the surrounding liquid, we can calculate the volume of the detected nanodroplets. To gain insight into dissolution process, we measured the effective area of each droplet by applying a Modified Voronoi analysis. The Modified Voronoi cells, defined as the distance to the footprint edge of the droplets [215], were calculated from the optical images by a custommade MATLAB function. Figure 7.3 a shows the Modified Voronoi (MVor) tessellation (black lines) on an optical image of surface nanodroplets. Each red circle gives the footprint of a surface nanodroplet with area of $a$, while its M-Voronoi cells with area of $A_{M}$ are displayed in white colour. Figure $7.3 \mathrm{~b}$ shows the correlation between the footprint areas $a$ and the M-Voronoi cell areas $A_{M}$, i.e. $A_{M} \propto a$, which suggests that surface nanodroplets form through heterogeneous nucleation and diffusive growth [215]. The dashed green vertical line indicates the optimal optical resolution for the lateral boundary detection, and $K^{2 / 3}$ is a prefactor. 


\subsection{Conclusions}

In summary, we theoretically and experimentally investigate the formation of surface nanodroplets by solvent exchange under well-controlled flow conditions. We found that although the contact angle of surface nanodroplets is independent of flow conditions, the flow rate and flow geometry have significant effects on the droplet size. We developed a theoretical framework for the solvent exchange process, whose result is in good agreement with the experimental results, namely that the droplet volume increases with $P e^{3 / 4}$. Increasing the channel height (for given flow rate and thus given $P e$ ) can induce convection driven by the density difference between water and ethanol, leading to larger droplets and an inhomogeneous droplet nucleation pattern, which reflects the convection rolls. The results presented in this work provides valuable guideline for the device design to generate surface nanodroplets with some desired sizes. 


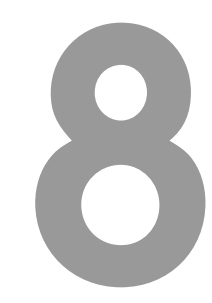

\section{D fitting procedure for mass of nanodroplets on flat surface or micro-caps* $*$}

In the study of nanobubbles, nanodroplets or nanolenses immobilised on a substrate, a cross-section of a spherical-cap is widely applied to extract geometrical information from atomic force microscopy (AFM) topographic images. In this paper, we have developed a comprehensive $3 D$ spherical cap fitting procedure (3D-SCFP) to extract morphologic characteristics of complete or truncated spherical caps from AFM images. Our procedure integrates several advanced digital image analysis techniques to construct a $3 D$ spherical cap model, from which the geometrical parameters of the nanostructures are extracted automatically by a simple program. The procedure takes into account all valid data points in the construction of $3 D$ spherical cap model to achieve high fidelity in morphology analysis. We compare our $3 D$ fitting procedure with the current $2 D$ cross-sectional profile fitting method to determine the contact angle of a complete spherical cap and a truncated spherical cap. The results from $3 D$ SCFP are consistent and accurate, while $2 D$ fitting is arbitrary in selection of a cross-section with large variation due to limited data points taken for the

${ }^{*}$ Based on: H. Tan, S. Peng, C. Sun, X. Zhang, and D. Lohse, 3D spherical-cap fitting procedure for (truncated) sessile nano- and micro-droplets \& -bubbles, The Europen Physical Journal E 39 (11), 106 (2016).

${ }^{\dagger}$ AFM images provided by S. Peng. 
fitting. We expect the developed $3 D$ spherical-cap fitting procedure will find many applications in imaging analysis.

\subsection{Introduction}

Atomic force microscopic (AFM) imaging has been one of the most popular techniques in the study of surface nanobubbles, nanodroplets and many other spherical cap nanostructures [37, 63, 199, 222]. Thanks to the high spatial resolution of AFM measurements, the size of nanobubbles and nanodroplets immobilised on the substrate can be characterised accurately in all three dimensions. Geometrical features of the spherical cap structures, such as lateral extension of the footprint, height and contact angle, can be compared on a quantitative level [37, 223 225]. Although most of those parameters are directly read out from the images, the contact angle of nanobubbles and nanodroplets requires further imaging analysis and data processing. As surface nanobubbles or nanodroplets are usually spherical caps due to dominance of capillarity on such small dimension, the data analysis requires the construction of an ideal spherical cap to fit the AFM data.

Up to now, fitting a two-dimensional (2D) cross-sectional profile is the most common approach for determining the contact angle of nanobubbles and nanodroplets [225 233]. With an AFM off-line software, the cross sectional profile through the centre of bubbles or droplets can be conveniently read out from the image. For instance, Simonsen et al. [228] and Wang et al. [230] calculated the contact angle of nanobubble, based on the measured height and footprint size in the cross-section. Wang et al. 2225] determined the droplet size by simply averaging the values calculated from several different crosssection profiles. In a slightly improved method, the cross-sectional profile is fitted with a portion of an ideal circle (i.e. an arc). The angle subtended by the arc and the flat baseline is the contact angle of the measured nanostructures. This method has been applied by Zhang et al. 226, 227, 232, 233] and Yang et al. 234] to obtain the contact angle of nanobubbles and nanodroplets on several flat substrates. However, the drawbacks from 2D fitting are the arbitrary selection of the cross-section, which leads to large variation in the contact angle measured along different directions in the image [225, 229].

To obtain accurate a contact angle, the contribution of all valid the data points on the nanostructure must be taken into account in the reconstruction of the spherical cap model. This requires the development of a comprehensive 3D fitting [235, 236]. Although Song et al. [236] applied a 3D spherical-cap fitting to analyse the morphology of nanobubbles, no 3D fitting procedure has been reported for the analysis of a truncated spherical cap on a substrate 
with physical structures. A simple example of truncated droplets is that the droplets form at the rim of a microcap [233. The shape of those truncated droplet resembles an apple slice with a part bitten off from the flat face. Furthermore, sensible exclusion of unreliable data points is an important aspect in 3D fitting which has not been considered so far. Particularly for highly curved bubbles or droplets, the data points close to the three-phase contact line should be excluded (i.e. contact angle larger than $90^{\circ}$ ), due to the tipsample convolution [37, 237]. In the analysis of an image of very small bubbles and droplets, there may be also potential effects from the disjoining pressure [238, 239], and hence only the data points above a certain threshold are valid for the fitting [235, 236].

In this paper, we have developed a 3D spherical-cap fitting procedure (3D$\mathrm{SCFP}$ ). The procedure employs the techniques of digital image processing to recognise the features from a spherical cap. It performs well for an isolated nanodroplet sitting on a flat substrate, but also for a truncated nanodroplet on the rim of a microcap [233, 240]. In the latter case, a feature extraction method, Circle Hough Transform, is performed to distinguish the AFM data points from the part of the underlying microcaps and from the truncated nanodroplets [221, 241, 242].

With our proposed 3D-SCFP, we will analyse polymerised nanodroplets (i.e. nanolenses) in AFM images collected from our previous work [240], as represented in Figure 8.1 a. The nanodroplets (indicated by blue solid arrows) to be analysed include both complete spherical caps on a flat area and truncated droplets by the underlying microstructures (indicated by red dashed arrow). 3D-SCFP will translate AFM data points to ideal spherical caps that nicely match the nanodroplet morphology, as displayed in Figure 8.1p. For those truncated nanodroplets, the procedure recognises the data points from a part of the spherical cap and reconstruct a whole spherical cap model.

The following sections of the paper are organised as following: Section 2 details the algorithm of 3D-SCFP, and section 3 discusses the influence of an important parameter, the threshold of height cut-off, on 3D fitted result. After that, the paper gives two examples in section 4, showing the comparison between 3D-SCFP and 2D cross-sectional profile fitting method. The results reveal the $3 \mathrm{D}-\mathrm{SCFP}$ is robust, compared to the $2 \mathrm{D}$ fit method. The Matlab codes of $3 \mathrm{D}-\mathrm{SCFP}$ are provided in the supplementary material, free access to the audience. 

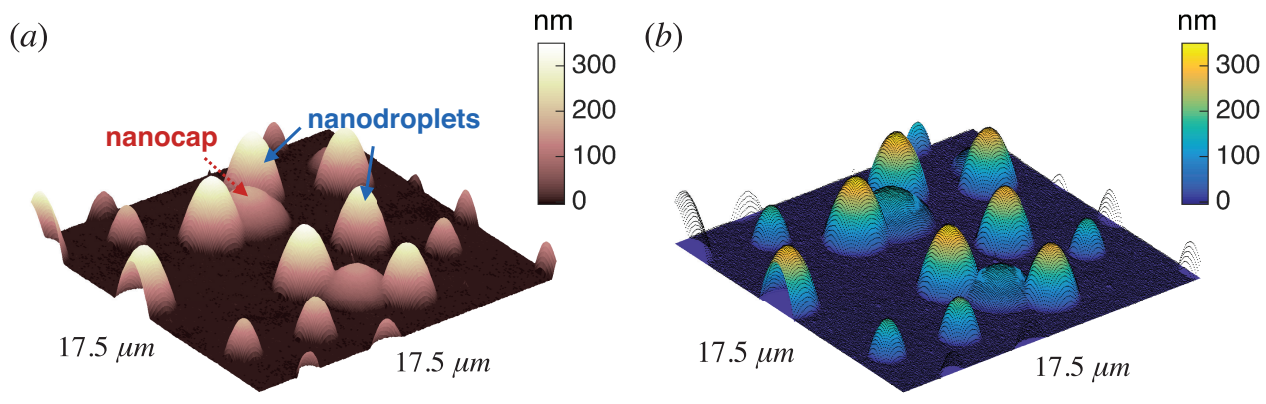

Figure 8.1: Three-dimensional representation of the nanodroplets and overlapped microcaps (the arrows). (a) AFM image of nanodroplets sitting on the flat substrate and on the rim of microcaps. Image size: $17.5 \mu \mathrm{m} \times 17.5 \mu \mathrm{m}$. (b) Reconstructed three-dimensional image from the 3D spherical-cap fitting procedure. The AFM data points are displayed as the black dots, showing a nearly perfect agreement with the fitting surface.

\subsection{Detailed 3D fitting procedure}

The algorithm consists of four parts: raw image preprocessing, objective detection, objective identification, and cut-off of vertical data points near the rim.

\subsubsection{Raw image pre-processing}

The AFM images pretreated by second-order flattening by AFM off-line software are used in this step. A representative image is shown in $I$ (Fig. 8.2 a). We create a gamma encoded image $I^{\prime}$ (Fig. $8.2 \mathrm{p}$ ) from the raw image $I$. Gamma encoding is defined as $V_{\text {out }}=A V_{\text {in }}^{\gamma}$ with $1>\gamma>0$, where $V_{\text {out }}$ and $V_{\text {in }}$ present pixel intensity of images $I^{\prime}$ and $I$ respectively, and $A$ is a constant. After the operation, the pixel intensity of the whole image $I^{\prime}$ is nonlinearised, resulting in a sharper contact line with the flat substrate. The obtained image is shown in Figure $8.2 \mathrm{~b}$. The sharp boundary in the image increase the reliability of the feature detection in next step 243 .

\subsubsection{Objective detection}

This step determines the circular footprint of an complete nanodroplet on the flat substrate, but also the footprints of multiple truncated nanodroplets and the underlying microcaps (blue circles in Fig $8.2 \mathrm{~b}$ ). An edge detection technique, Circle Hough Transform [221, 241, 242], is applied to the gamma encoded image $I^{\prime}$. In order to have all the data points of a complete droplet included in the circle of the footprint, we set a parameter $p a_{0}$ to adjust the radii of the fitting. As demonstrated in the zoom-in in Figure $8.2 \mathrm{k}$, the initially detected circle (blue) doesn't envelop all the data points of a nanodroplet. By 

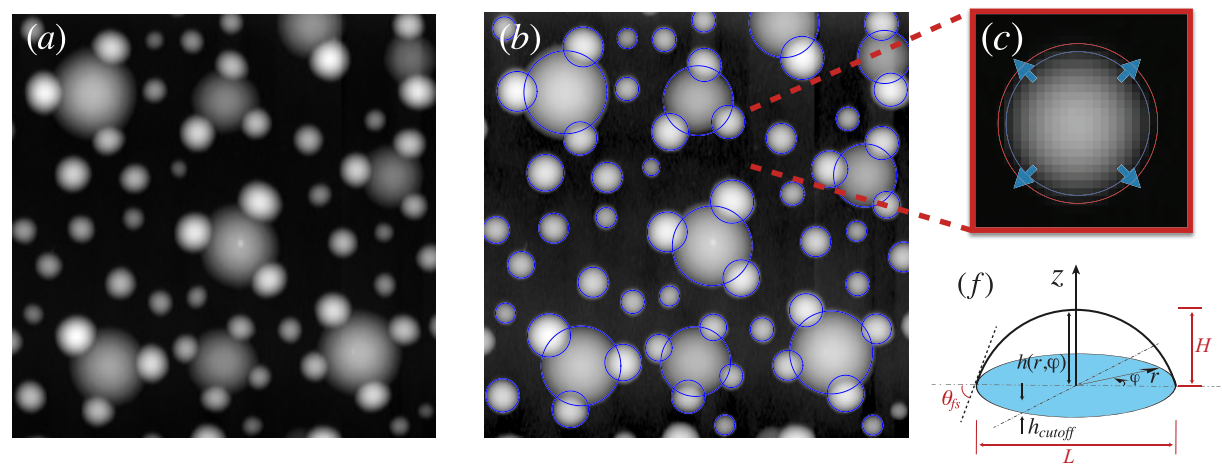

$(d)$
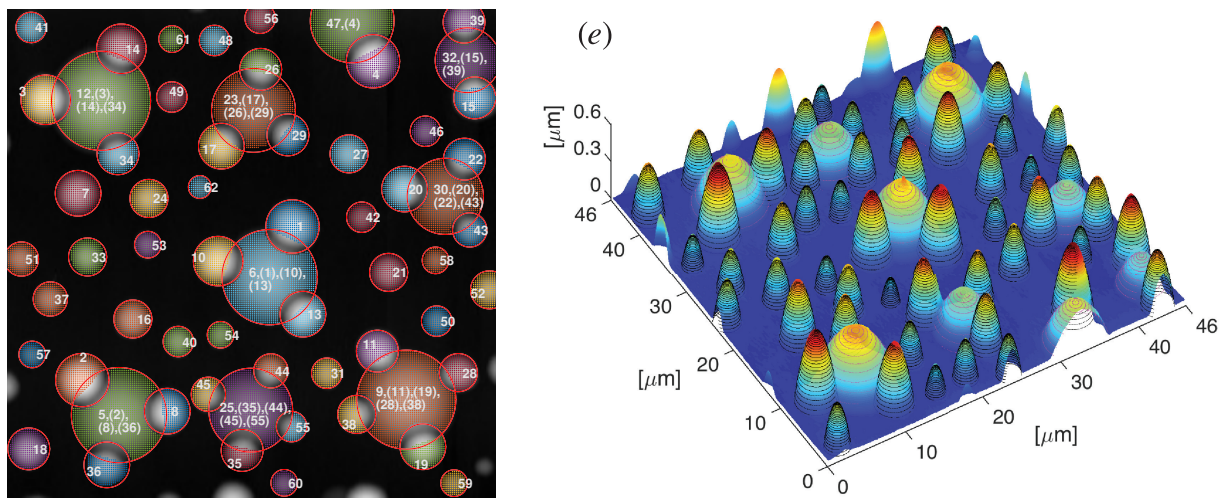

Figure 8.2: Screen snapshot series of the $3 \mathrm{D}$ spherical-cap fitting procedure (a-e). (a) $2 \mathrm{D}$ representation of an AFM sample image. The pixel intensities of the graphics correspond to the sample height. (b) In the second step of the procedure, the footprints of the objectives are detected as blue circles. In order to surround all the data points (pixels) of each objective, the radii of the circles are designed to be adjustable as shown in (c). (d) shows the identification of the objective with coloured data points for 3D fit in the final step. The relation between all the nanodroplets and the overlapped microcaps is also recognised. (f) The final fitted ideal geometries (the dot lines), showing a very good agreement with corresponding AFM sample image in 3D display (the coloured surface). (d) The sketch of an isolated nanodroplet with annotations. The $h_{\text {cutof } f}$ is defined as the height respect to the flat substrate.

increasing the radius, a new circle (red) is generated, now wrapping up all data points (pixels) in the image. The rim of the truncated nanodroplet on the flat substrate is part of circle, which together with the rim of the underlying microcap can be detected through advanced feature extraction method, Circle Hough Transform. As shown in Figure $8.2 \mathrm{~b}$, those blue connected rings fit the boundaries of microcap and the truncated droplets nicely.

\subsubsection{Objective identification}

If there are only isolated nanodroplets in the AFM image, the step discussed in this subsection is not essential. For the case with nanodroplets sitting on the 
rim of a microcap, it is crucial to automatically identify weather the detected objective is a nanodroplet or a microcap. The identification is based on the characteristics from experiments: (i) Nanodroplets can sit above microcaps, while the reverse does not apply; (ii) Serval nanodroplets can nucleate on the rim of an identical microcap; (iii) The truncated nanodroplets are higher or lower than the microcaps, depending on their materials (details refer to Ref. [240]).

Based on those information, we perform some conditional statements to determine the nanodroplet and the microcap. Then the data points in the overlap region are attributed to the recognised nanodroplets according to characteristics (i) (see appendix A for the details). In Figure 8.2 d, the identification numbers are displayed. Behind the label number of each microcap, the label numbers of its overlapping nanodroplets are also listed in the parentheses.

\subsubsection{Cut-off of vertical data points near the rim}

As already mentioned in Introduction, the data points near the rim are subject to the influence of tip convulsion and the disjoining pressure. Here we take those data points above a threshold height for 3D spherical cap fitting (details are discussed in section 8.3). The threshold is a new parameter, $h_{\text {cutoff }}$ (sketch in Fig 8.2f). The valid data points are shaded in different colours in Figure $8.2 \mathrm{~d}$, and fitted with a spherical cap by the program. The fitting is generated by minizing the cost function, which is the summed distance from the data points to the ideal spherical surface. The system of the nonlinear equation for the optimisation is directly solved by MATLAB. As shown in Figure 8.2e, the whole topographic AFM image (the coloured surface) is perfectly matched by the corresponding ideal spheres (the black dot lines for nanodroplets and red for microcap). The fitting results are saved as ideal spheres' radii and their centre-point coordinates with respect to the flat substrate surface. Based on these parameters, the calculations of footprint lateral extension $L$, height $H$, contact angle on flat substrate $\theta_{f s}($ Fig. $8.2 \mathrm{f})$, contact angle on micro-cap $\theta_{m c}$, and other relevant geometrical parameters are determined mathematically.

\subsection{Evaluation of the effects from cut-off threshold}

The selected threshold value $h_{\text {cutoff }}$ is import for the consequent contact angle obtained by 3D-SCFP. In this section, we show the effect of the threshold by analysing the droplet in Fig. 8.4 a. We apply 3D-SCFP to this nanodroplet with 31 different $h_{\text {cutoff }}$ in the range from $1 \mathrm{~nm}$ to $30 \mathrm{~nm}$ (around $10 \%$ height of the nanodroplet), while all the other fitting parameters are fixed. The result displayed in Figure 8.3 a shows that when the threshold $h_{\text {cutoff }}$ increases, 
(a)

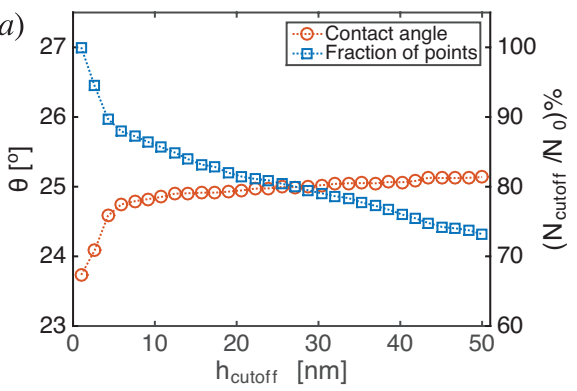

(b)

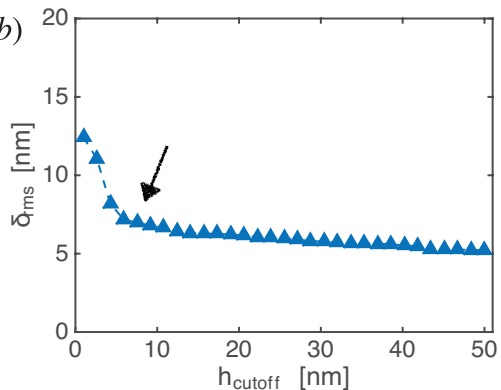

Figure 8.3: (a) The contact angle and the fraction of the corresponding fitted data points versus the cut-off height $h_{\text {cutoff }}$ in $3 \mathrm{D}$ spherical-cap fit. When $h_{\text {cutoff }}$ is smaller than a certain value (around $10 \mathrm{~nm}$ ), the fitted contact angle has a relatively large variation. (b) The fit error $\delta_{r m s}$ versus the cut-off height $h_{\text {cutoff }}$ in 3D spherical-cap fit. The fit error $\delta_{r m s}$ has a sudden increase when $h_{\text {cutoff }}<10 \mathrm{~nm}$, indicating that the data points below 10 $\mathrm{nm}$ are questionable for $3 \mathrm{D}$ spherical-cap fit. The $3 \mathrm{D}$ representation of the nanodroplet is shown in Figure 8.4(a).

both the fraction of data points above it (with respect to the number of the data points above $1 \mathrm{~nm}$ ) and the 3D fitted contact angle vary. The fraction decreases as expected while the contact angles $\theta$ shows a strong dependence on $h_{\text {cutoff }}$ for $h_{\text {cutoff }}<10 \mathrm{~nm}$. In Figure $8.3 \mathrm{~b}$, we define the fit error $\delta_{\text {rms }}$ as root mean square of the radial distance between the data points and the ideal sphere, showing the great contribution to fit error from the data points close to the substrate.

According to evaluations of the contact angle and the fit error, an appropriate cut-off must be determined slightly after the turning point. Too small $h_{\text {cutoff }}$ causes large 3D spherical-cap fitting error. On the other hand, too larger $h_{\text {cutoff }}$ leads to less contributions from valid data points.

\subsection{D-SCFP versus 2D fitting}

We provide in this section the comparison between the results of 3D-SCFP and 2D cross-sectional profile fitting method in two cases: an isolated nanodroplet and two truncated nanodroplets.

\subsubsection{Case1: An isolated nanodroplet on the flat substrate}

The selected data points used to be fitted are above $10 \mathrm{~nm}$ in height, which has been properly determined as described in section 8.3 (Fig. 8.3 $\mathrm{a}$ ). In figure 8.4 a, the 3D fitting result (the coloured spherical surface) shows an excellent agreement to the AFM data points (the black dots). The contact angle $\theta_{f s}$ calculated from fit is listed in Table 8.1 
(a)

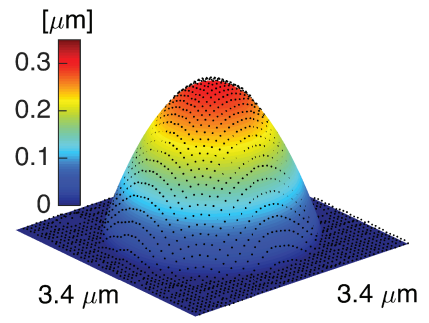

(b)

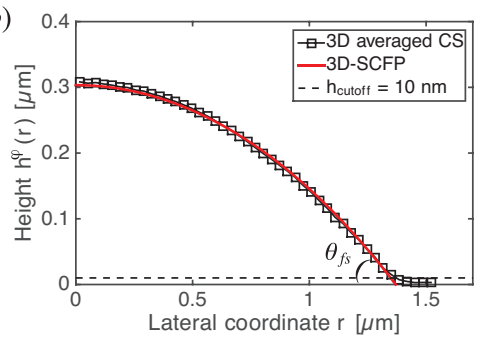

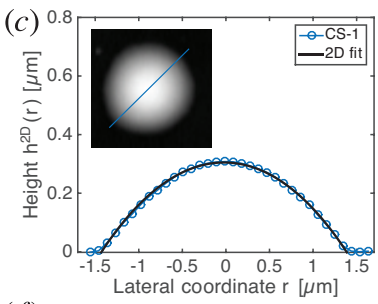

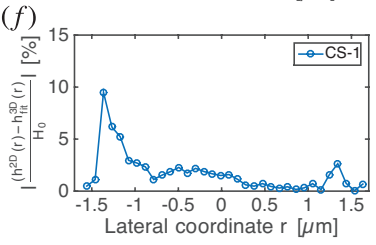

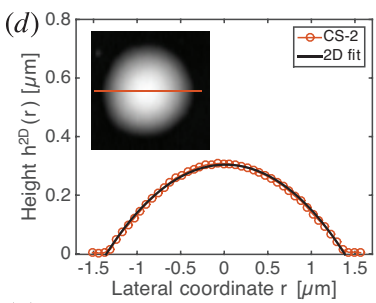

$(g)$

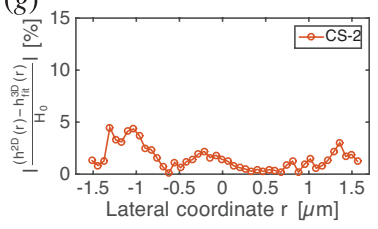

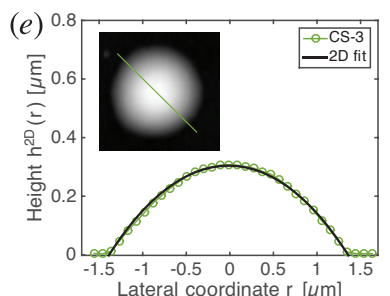

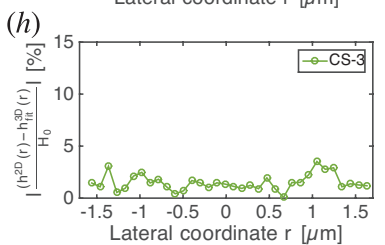

Figure 8.4: (a) Reconstructed 3D image of an AFM sample image, in which an isolated nanodroplet sitting on the flat substrate. The AFM data points displayed as the black dots fit well with the coloured ideal spherical surface calculated from 3D-SCFP. Image size: $3.4 \mu \mathrm{m} \times 3.4 \mu \mathrm{m}$. Droplet height: $0.3 \mu \mathrm{m}$. (b) Defined averaged cross-sectional profile $h^{\varphi}(r)$ (square), showing an excellent agreement to the 3D fitting result (red curve). (c-e) Three cross-sectional profiles CS-1, CS-2 and CS-3 of the same nanodroplet extracted along different directions. The cross section cutting lines are shown in the inserted AFM images, respectively. Based on the spherical-cap assumption, the fitting shapes (the black curves) match the three different cross-sectional profile (circle) very well, but give different fitted contact angles (Tab. 8.1). Corresponding to (c-e), (f-h) show the deviation of each profile $h^{2 D}(r)$ from its ideal spherical-cap fitting result $h_{f i t}^{3 D}(r)$, which considers the contributions of all the data points.

We introduce an averaged cross-sectional profile $h^{\varphi}(r)$ defined as:

$$
h^{\varphi}(r)=\frac{1}{2 \pi} \int_{0}^{2 \pi} h(r, \varphi) d \varphi,
$$

where $h(r, \varphi)$ is the height of the data point in the cylindrical coordinates (sketch Fig. 8.2F). The origin of the coordinate system is the centre point of the circular footprint of the ideal sphere on the flat substate. As displayed in Figure 8.4 , the 3D spherical-cap fitting result (the red line) and the averaged cross-sectional (the black square line) profile perfectly match with each other as expected. 
In 2D fitting, the nanodroplet is assumed to be an ideal spherical cap. Part of three circles are used to fit three different cross-sectional profiles of the same nanodroplet: CS-1 (along $45^{\circ}$ direction), CS-2 $\left(0^{\circ}\right)$ and CS-3 $\left(135^{\circ}\right)$, as shown in Figures 8.4 , d and e, respectively. It is notable that the profile in CS-2 has more data points (pixels) than others, because CS-2 is along horizontal direction of pixel matrix in the AFM image. The fitting results show that the 2D fitting circle (the black line) perfectly matches the profile data (the circular dots) in each cross section. Theoretically, the fitted contact angles $\theta_{f s}$ of different cross-sectional profiles should be the same. But the contact angles fitted from those three profiles data are different, as listed in Table 8.1. $\theta_{f s}$ obtained from CS-1 is smaller than the fitted results of other two cross-sectional profiles, due to a variation among the profiles in different cross sections. To reveal where the difference comes from, we introduce a local deviation defined as $\left|\left(h^{2 D}(r)-h_{f i t}^{3 D}(r)\right) / H_{0}\right|$. It is the difference between the extracted cross-sectional profile data $h^{2 D}(r)$ and the corresponding ideal 3D profile $h_{f i t}^{3 D}(r)$, normalised by the height of the nanodroplet $H_{0}$. The introduced definition displayed in Figure 8.4 -h clearly indicates that the departure comes from the profile part that near the contact line. The largest departure in CS-1 results in the greatest deviation of the fitted contact angle from others $\theta_{f s}$ (Tab. 8.1), while the CS-2 and CS-3 with the small departure along the whole profile (Fig. $8.4 \mathrm{~g}$ and $\mathrm{h}$ ) give the similar $\theta_{f s}$ value as the one calculated from the 3D fit. It is also found that the departures are asymmetry and its magnitude varies in different cross sections. This may be due to a typical issue associated with long time scanning required to collect an image. The draft from the previous scanned area may introduce inaccuracy in the lateral size. Another possibility is that the droplet is never an ideal spherical cap, due to the unavoidable chemical heterogeneities on the surface [20, 244]. Overall, the variation of the extracted cross-sectional profile jeopardise the reliability of $2 \mathrm{D}$ cross-sectional profile fitting method.

\subsubsection{Case2: Truncated nanodroplets sitting on the rim of a microcap}

In the second example, we test a complex case with two truncated nanodroplets $\mathrm{A}$ and $\mathrm{B}$ sitting on the rim of a microcap C (Fig. 8.5 $\mathrm{a}$ ). The parameter $h_{\text {cutoff }}$ is determined as $10 \mathrm{~nm}$ beforehand. 3D-SCFP programmatically distinguishes the data points of the two truncated nanodroplets A, B and the overlapped microcap C. Then it successfully fits valid data points of each them to an ideal sphere. In the Figure 8.5 $\mathrm{a}$, an excellent agreement between the fitting result (coloured spherical surface) and AFM data points (black dots) is obviously displayed. Based on the definition in Equation 8.1, we also build their averaged cross-sectional profiles. Three averaged cross-sectional profiles (black square) 
Table 8.1: Comparison of the calculated contact angles through 3D-SCFP and 2D crosssectional profile fitting method. The cross section labels correspond to those in Figures 8.4 and 8.5. Two kinds of contact angle are compared: $\theta_{f s}$, contact angle of nanodroplets or microcap on flat substrate, and $\theta_{m c}$, contact angle of the nanodroplet on a microcap. $\mathrm{d}$ is short for nanodroplet and $\mathrm{c}$ is short for microcap.

\begin{tabular}{cccccccc}
\hline & CS-1 & CS-2 & CS-3 & CS-4 & CS-5 & CS-6a,b,c & 3D fit \\
\hline$\theta_{f s}$ & $24.48^{\circ}$ & $25.10^{\circ}$ & $25.02^{\circ}$ & & & & $24.98^{\circ}$ \\
a: $\theta_{f s}(\mathrm{~d})$ & & & & $29.08^{\circ}$ & & $26.80^{\circ}$ & $26.99^{\circ}$ \\
a: $\theta_{m c}(\mathrm{~d})$ & & & & $30.73^{\circ}$ & & & $28.87^{\circ}$ \\
b: $\theta_{f s}(\mathrm{~d})$ & & & & & $27.45^{\circ}$ & $27.64^{\circ}$ & $27.82^{\circ}$ \\
b: $\theta_{m c}(\mathrm{~d})$ & & & & & $29.35^{\circ}$ & & $30.28^{\circ}$ \\
c: $\theta_{f s}(\mathrm{c})$ & & & & $7.16^{\circ}$ & $7.52^{\circ}$ & $7.81^{\circ}$ & $7.65^{\circ}$ \\
\hline
\end{tabular}

are integrated in a height-radial coordinate with the origin defined as the footprint centre of the microcap $\mathrm{C}$ (Fig. 8.5b). The 3D fitting results are displayed as the red lines, showing perfect agreement to the averaged profiles. Here, we introduce the contact angle of the nanodroplet on the overlapped microcap, labeled as $\theta_{m c}$. Then both $\theta_{f s}$ and $\theta_{m c}$ are calculated from 3D fitting results and are listed in Table 8.1 .

For this case, it is no longer convenient to apply $2 \mathrm{D}$ cross-sectional profile analysis any more. To obtain the correct contact angle $\theta_{m c}$, we must pick up the cross section that passes both the centre point of the nanodroplet and that of the microcap. However, there are unavoidable artificial errors of locating the centra positions of the nanodroplet and the microcap. When the cutting line of the selected cross section is not in the column direction or row direction of the image pixel matrix, the profile of an selected cross section can not be precisely depicted, because the cutting line may not pass pixel centres (as sketched in Fig. 8.7 in appendix B). In low pixel resolution image, this problem is even more crucial. In order to reduce those influence as much as possible in this section, firstly we choose an AFM image with a relatively high resolution. The lateral dimension of a nanodroplet is depicted by more than 40 pixels (Fig. 8.5 a). Secondly, the extraction of the cross-sectional profile in a particular direction is improved by applying a linear fitting, as described in appendix B.

In Figures 8.5;, d and e, two cross sections (CS-4 and CS-5) that pass two centre points of the objectives and three complete cross-sectional profiles of each objective (CS-6a, b and c) are displayed. Notably, the profile of microcap $\mathrm{C}$ in CS-4 is not only overlapped by nanodroplet $\mathrm{A}$, but also slightly by the edge of nanodroplet $\mathrm{B}$, which is not conspicuous. The overlap reduces the number of the valid data points for $2 \mathrm{D}$ fit. This situation tends to be common when 
(a)

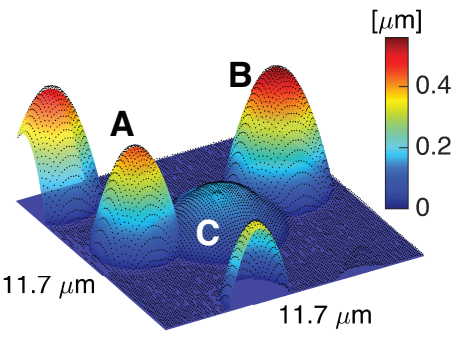

(b)

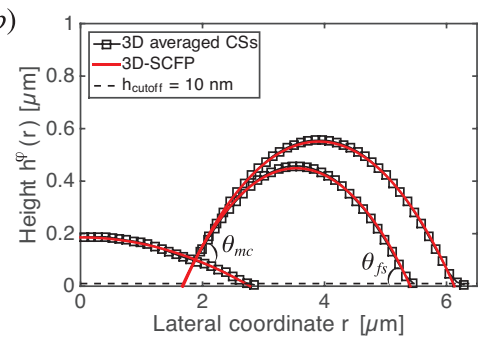

(c)
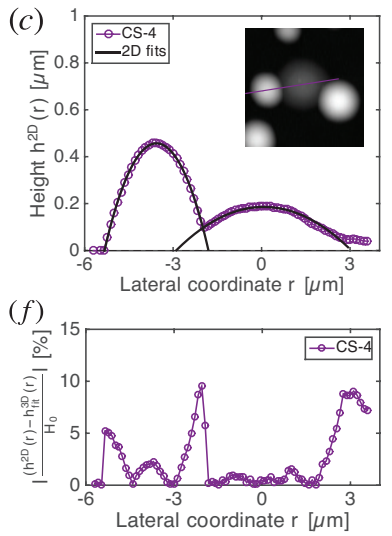
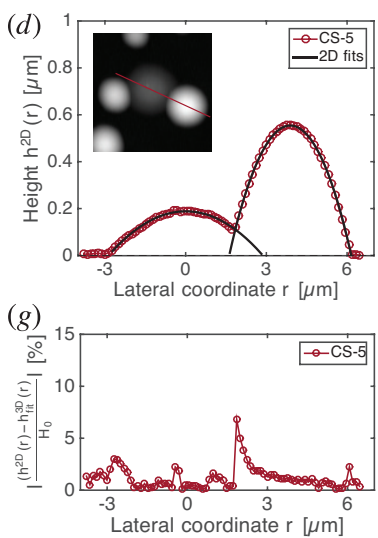

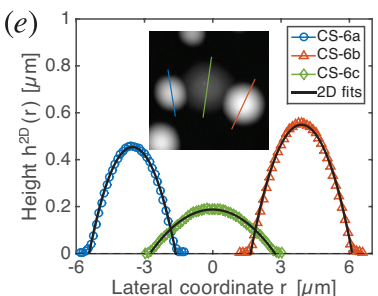

(h)

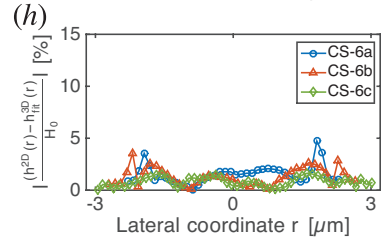

Figure 8.5: (a) Reconstructed 3D image of an AFM sample image, in which two nanodroplets $\mathrm{A}$ and $\mathrm{B}$ sitting on the rim of a microcap $\mathrm{C}$. The AFM data points displayed as the black dots fit well with the coloured ideal spherical surface calculated from 3D-SCFP. (b) Defined averaged cross-sectional profiles $h^{\varphi}(r)$ (square), showing an excellent agreement to the 3D fitting results (red curves). The positions of the nanodroplets and the microcap are based on the centre distances. (c-h) hold the same definitions as in Figure 8.4 (c-h) (the calculated contact angles refer to Tab. 8.1.

there are more nanodroplets sitting on a same microcap. We only apply $2 \mathrm{D}$ fits to the valid segment of the extracted profiles. As shown in Figures. 8.5-e, the 2D fitting results (the black circles) have a good agreement to the corresponding profile data. Based on the 2D fitting results, $\theta_{f s}$ and $\theta_{m c}$ of the nanodroplets and the $\theta_{f s}$ of the microcap are calculated and are listed in Table 8.1. The normalised deviation of $h^{2 D}(r)$ from $h_{f i t}^{3 D}(r)$ is calculated and is displayed in Figures $8.5 \mathrm{f}$-h. It shows that the deviation increases in the profile part nearby the contact point/line. Especially for nanodroplet A in CS-4, its profile severely departs from its 3D fitting profile (Fig. 8.5f), leading to $2^{o}$ difference for both $\theta_{f s}$ and $\theta_{m c}$ (Tab. 8.1). However, with the partial profile of nanodroplet B in CS-5, we obtain a similar 2D fitted $\theta_{f s}$ and $\theta_{m c}$ with the 3D-SCFP results. We can also acquire similar fitted result $\theta_{f s}$ when apply 2D fit to the complete extracted profiles CS-6a, b and c. But the contact angle $\theta_{m c}$ is unaccessible from these complete profiles. In a conclusion, the 
$2 \mathrm{D}$ cross-sectional profile fitting method loses its convenience features and is more sensitive to image pixel resolution. The inconsistency of the $2 \mathrm{D}$ fitting results is becoming more obvious. In other words, 3D-SCFP demonstrates its incomparable superiority in this complex case.

\subsection{Conclusion}

In this paper, we provide a 3D spherical-cap fitting procedure (3D-SCFP) with further advantages and capability. The procedure integrates powerful feature extraction method - Circle Hough Transform. Through this method, the data points of the truncated nanodroplets and the overlapped microcaps can be accurately separated. Then the procedure applies 3D fit to the nanodroplet and the microcap separately. The details of the procedure and the MATLAB program are provided. We also provide comparison between the result of 3D spherical-cap fitting procedure and that of the classical 2D cross-sectional profile fitting method in two AFM images: one with an isolated nanodroplet and another complex one with two truncated nanodroplets sitting on a microcap. The result shows that the drawback of $2 \mathrm{D}$ fit lies in the small amount of fitted data points, the artificial error of the cross section selection and the possible distortion of the extracted profile nearby the contact line. All those lead the inconsistent results of $2 \mathrm{D}$ cross-sectional profile fitting method. When an required cross section is not along the pixel array in the second case, it is difficult to directly extract an accurate cross-sectional profile. However, the 3D-SCFP takes consideration of all the valid data points and $2 \mathrm{D}$ profile extraction is not necessary any more. It performs well for both cases and gives consistent and accurate 3D fitting results. Therefore the 3D-SCFP is strongly preferable. The method and codes created in this paper are readily implemented for imaging analysis of similar nanostructures, and are accessible to all users through the supplementary materials online.

\subsection{Appendixes}

\subsubsection{Appendix A. Data points division}

The projector of the contour of the truncated nanodroplet on the top surface of microcap (the black solid line) is a part of an ellipse. It is within the overlapping region of two detected circles. In "Objective detection" step of 3D-SCFP, we prepare data points inside the microcap-circle and outside the nanodropletcircle (Data group 1) for 3D fit of the microcap, while the data points inside 
the nanodroplet-circle and outside the microcap-circle (Data group 2-1) for $3 \mathrm{D}$ fit of the nanodroplet.

In order to also consider contributions from the data points in the overlapping region, we divide the region into two parts with a straight line (the red dash-line). The line passes through two intersection points of two circles. Once the nanodroplet is recognised in "Objective identification" step, we can determine the part that all belongs to nanodroplet. The part (Data group 2-2) is labeled with red-line pattern as displayed in Figure 8.6. Hence, both the data group 2-1 and the data group 2-2 are used in 3D fit of the overlapping nanodroplet in the final step of 3D-SCFP.

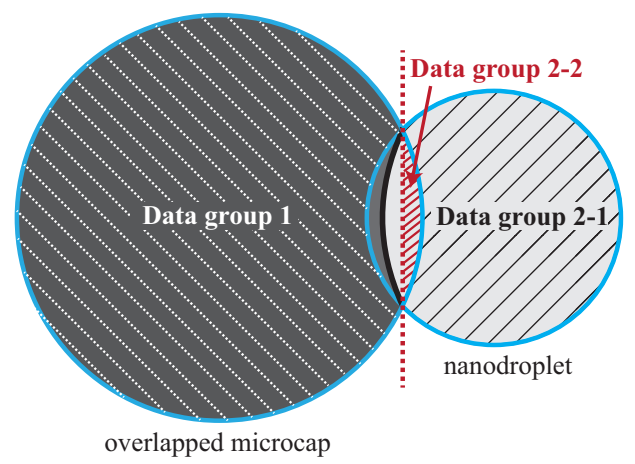

Figure 8.6: Schematic diagram of dividing the joint data points of a nanodroplet (the light grey geometry) and the overlapped microcap (the dark grey geometry). Two blue circles, detected by Circle Hough Transform, provide two regions: Data group 1 (with the pattern of white diagonal dot-line) and Data group 2-1 (with black-diagonal-line pattern). The red dash-line passes the intersection points and defines the region of Data group 2-2 (with red-line pattern).

\subsubsection{Appendix B. Cross-sectional profile extraction}

AFM image is a raster image. Each pixel has a relative $\mathrm{x}-\mathrm{y}$ position in the image. The pixel intensity correspond to the sample height (or cantilever deflection) at that location. When we extract a cross-sectional profile along any direction except $0^{\circ}, 45^{\circ}$ or $90^{\circ}$, it is common to come across the situation shown in Figure. 8.7. The cutting line of the cross section does not pass through any pixel centres in a pixel column (row). In order to obtain the height value $I_{f}$ at position $\left(x_{0}, y\right)$, we assume there existing a linear variation between the height values at positions $\left(x_{0}, y_{i}\right)$ and $\left(x_{0}, y_{i+1}\right)$. Hence, $I_{f}$ is calculated as below,

$$
I_{f}=\frac{l_{B} I_{A}+l_{A} I_{B}}{l_{A}+l_{B}},
$$


where $I_{A}$ and $I_{B}$ are the distances from the investigating point to the neighbour pixel centres. The AFM image used in Figures 8.5 have high resolution, so the linear fit is precise enough. For low resolution AFM image, it is required to apply high order fit which considers contributions from more neighbour pixels.

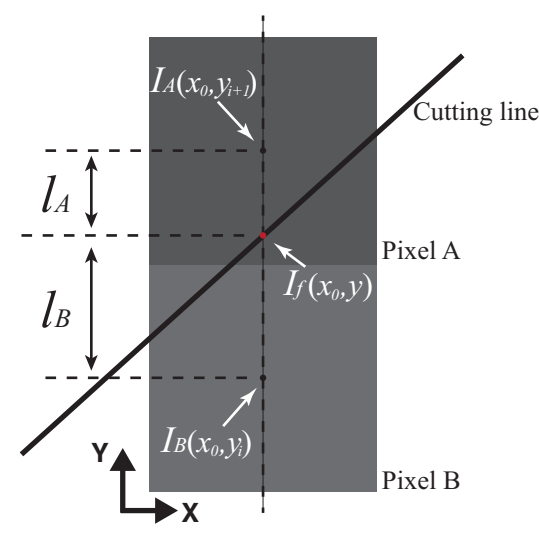

Figure 8.7: Schematic diagram of extracting an arbitrary line from a pixel-array/data-matrix. Two big solid squares represent two pixel points $\mathrm{A}$ and $\mathrm{B}$ with corresponding recorded signal (sample height in the pixel centre) $I_{A}$ and $I_{B}$. When a line doesn't pass through the centre of the pixel, the grey values is $I_{f}$. 


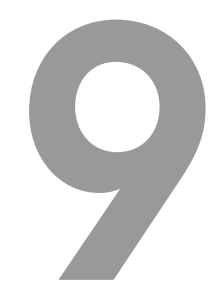

\section{Conclusions and outlook}

In this thesis, we have studied the dynamical behaviors induced by the "ouzo effect" in evaporating and dissolving multicomponent droplets through a combined experimental-numerical-theoretical approach. During this study, we not only discovered and further explained many amazing and unexpected phenomena, which might drawn interests from a variety of fields, but also applied one of these phenomena in supraparticle fabrication. In the end the surface nanodroplets were discussed.

In $\S 2$, we studied the evaporation of the sessile ouzo droplet on an octadecyltrichlorosilane (OTS)-glass surface through a variety of experimental technologies and numerical simulations. In this study, we revealed and theoretically explained the four different phases of the droplet life: In phase I, the ouzo droplet appears transparent due to a large fraction of ethanol in the ouzo solution. The high volatility of ethanol causes its concentration reduction in the droplet, preferentially at rim of the droplet due to the singularity there. Thus the phase separation initially takes place at the contact line. This is the beginning of phase II, in which oil microdroplets quickly nucleate in the whole drop, leading to its milky color which typifies the so-called "Ouzo-effect." During the evaporation, the nucleated oil surface droplets merge up into an oil ring, which consequently cause a characteristic non-spherical-cap shape. In the first two phases, there are intense solutal Marangoni flows inside the droplet due to concentration gradients. The phase III starts when the ethanol has evaporated out. A water drop sits on the oil-ring and the solutal Marangoni flows stop. In phase III, the droplet has a distinctly lower evaporation rate 
than in the first two phases due to the absence of the ethanol evaporation, which is clearly proved in our simulations. Finally, in phase IV, also all water has evaporated out, leaving behind a tiny spherical cap-shaped oil drop. Since the divergence of the local evaporation rate at the contact line is essential to the preferred evaporation-triggered "ouzo effect" at the droplet rim, we were curious about what would happen to an evaporating ouzo droplet without the divergence. This triggered the work in $\S 3$, where we used a superamphiphobic surface, which is both superhydrophobic and superoleophobic, to obtain a spherical ouzo droplet. Based on the evaporation theory, the divergence of the local evaporation rate disappears for the droplet with a Young's contact angle higher than $90^{\circ}$. The work in this chapter experimentally and numerically proved the absence of the preferred evaporation-triggered "ouzo effect" at the droplet contact line for a spherical ouzo droplet. Now, the preferred position moves to the top of the droplet because of the maximum evaporation rate there. The comprehensive simulations with the Finite Element Method helped us to notice the importance of the evaporative cooling effect for a droplet with high contact angles. Taking advantage of the existence of the intense solutal Marangoni flows, we assumed an isothermal field inside the droplet and corrected the evaporation model with an overall reduced temperature of the droplet. Meanwhile, Raoult's law was applied to express the interactions between different species at the vapor-liquid interface. With these assumptions, the generalized diffusion model successfully reproduced the experimental data. We also discovered an interesting phenomenon, in which the separated-out oil phase wraps up the droplet in the end, and explained it by calculating the spreading coefficient.

In $\S 5$, we have experimentally presented the rich phenomena of waterethanol drops dissolving in oil as host liquid. In this system, we observed w-in-o emulsification outside the drop, o-in-w emulsification inside the drop, buoyancy-driven convection dominating outside the drop, and prevailing solutal Marangoni convection inside the drop. To understand the diffusion-induced emulsifications, we developed a one-dimensional multi-diffusion model, which incorporates thermodynamical equilibrium theory and diffusion path theory, and successfully predict the experimental observations: diffusion-triggered win-o emulsification occurs when the drops have a ethanol content higher than 51.98 vol\%; o-in-w emulsification cannot be induced by pure diffusion. The model is only applicable for the early stage of the multiphase diffusion process due to the infinite domain and the Dirichlet boundary conditions used in this model. In practice, the continuous reduction of ethanol and increasing of oil in the drop lead to the occurrence of the o-in-w emulsification in a long time, which is thereby independent of the initial ethanol content of the drop. We 
also performed a scale analysis and PIV measurements to gain insight into its influence on the emulsification process by the diffusion-induced flow motions. Through the scale analysis, we demonstrated that in drop domain, the solute Marangoni flow prevails over the buoyancy-driven flow, while in host liquid domain, the latter dominates. This work provided a systematic investigation on the emulsification triggered by the dissolution of multicomponent drop in a host liquid. We expect that the proposed multi-diffusion model will have an important role in the study of the locomotion of dissolving multi-component droplets.

In the last chapter of Part] we provided a detailed investigation on the evaporation of mixture droplets with numerical and experimental methods. We first validated the axisymmetric finite element method model by Diddens et al. [111] by providing the good agreement of numerical predictions and the experimental data for a pure water droplet and a binary water-ethanol droplet. It has been shown that the quality of the agreement decisively depends on the consideration of the interplay of multi-component evaporation and thermal effects. While the pure water droplet and the binary waterethanol droplet are in perfect agreement with the experiment, the simulation of the ternary Ouzo droplet initially shows good agreement, including the onset of oil nucleation, but exhibits a faster evaporation of the remaining water residual than in experiment. This issue can presumably be attributed to the presence of the oil ring, i.e. a geometric deviation from the typical spherical cap shape, which was not included in the present model. To experimentally investigate the flow in the binary water-ethanol droplet, micro-PIV measurements have been performed. Since the flow is clearly non-axisymmetric as long as ethanol is present, the model was generalized to a full three-dimensional version. While the data is initially in good quantitative agreement, deviations between experiment and simulation can be found at later times. In particular, the simulation shows an intense thermal Marangoni flow once pure water remains, whereas the micro-PIV measurement shows no flow at all. Since all other aspects, i.e. composition-dependent liquid properties and thermal influences, are considered in the model, the mismatch can only be attributed to the presence of surface-active contaminations. Although we were unable to perform micro-PIV measurements in the Ouzo droplet, the flow inside this droplet was qualitatively revealed by the use of confocal microscopy. By visualizing the trajectories of the oil microdroplets, similar flow transitions as in the micro-PIV results of the binary water-ethanol droplet were found.

Inspired by the evaporating phenomena observed in the study of $\S 2$ in $\S 6$, we proposed a new method for supraparticle synthesis by applying an "ouzo" solution in evaporation-driven particle assembly. In our method, the evapo- 
rating colloidal drop was lubricated by its self-formed oil ring during the selfassembly of supraparticles, and therefore we call it "self-lubrication." Through confocal microscope technique, we clearly observed and physically explained the dynamics of the whole self-lubrication process during the evaporation. The presence of the oil ring from the merging of surface oil microdroplets plays a significant role in preventing the formation of a sticky contact line during the particle assembly. By varying the ratio of oil volume fraction to nanoparticles volume fraction in the initial colloidal solution, we generated supraparticles in a variety of shapes with high porosity. We proposed a simple method to explain the transition between ball-like shape and non-ball-like shape of the generated supraparitcles. The high porosity of the generated supraparticles was confirmed through the investigation of the inside structure by cutting the supraparticle slide by slide. Our new method provides huge improvements on evaporation-driven supraparticle self-assembly thank to its advantages of scalability, improving its operability and cost-efficiency, and flexibility, allowing for different sizes \& porosity. We expect that combing our method with piezoacoustic inkjet technology can dramatically scale up fabrication, as there is no limitation on supraparticle collection. Through this route, we expect more complex and interesting supraparticles can be generated by tuning the properties of (multi-) particles, as we observed that the nucleated oil microdroplets in the colloidal drop have the potential to organise particles locally.

In the last part, we drew our attention to the nucleated surface nanodroplets by the non-standard "ouzo effect". We used the solvent exchange method to form the surface nanodroplets in a narrow channel with controlled flow conditions. In $\S 7$, we experimentally and theoretically investigated the influence of the flow conditions on the formation of these nanodroplets. From our experimental images, there are significant effects from the flow rate and the flow geometry on the droplet size, although the contact angle of the nanodroplets is independent of the flow condition. We developed a theoretical framework to account for these effects based on the main idea that the droplet nuclei are exposed to an oil oversaturation pulse during the exchange process. The theoretical model predicts that the volume of the nanodroplets increases with the Péclet number Pe of the flow as $\propto P e^{3 / 4}$, which is in good agreement with our experimental results. In addition, at fixed flow rate and thus fixed Péclet number, convections originating from the density difference between the two solutions of the solvent exchange at wider channels cause larger and less homogeneously distributed droplets to form. We believed that the understanding from this work will provide valuable contributions for producing surface nanodroplets with desired sizes by controlling the flow conditions.

In $\oint 8$, we focused on the accurate extraction of morphological features 
from atomic force microscopy topographic images, which plays a vital role in surface nanodroplets study. We have developed a comprehensive 3D spherical cap fitting procedure (3D-SCFP) to extract morphologic characteristics of complete or truncated spherical caps from AFM images. The procedure integrates powerful feature extraction method - Circle Hough Transform, through which the data points of the truncated nanodroplets and the overlapped microcaps can be accurately separated. Then the procedure applies 3D fitting to the nanodroplet and the microcap data separately. We compared our 3D fitting procedure with the currently common-used 2D cross-sectional profile fitting method by calculating the contact angles of some complete spherical caps and truncated spherical caps. The comparison showed that the results from 3D- SCFP are consistent and accurate, while 2D fitting method gives results with large variations due to its arbitrary selection of the cross-sections and the limited number of the data points for fittings. Therefore the 3D-SCFP is strongly preferable. The method and codes created in this paper are readily implemented for the imaging analysis of the nanostructures with similar morphological features.

In the context of this thesis, the beauty and richness of the involved physics, chemistry, and fluid dynamics in small droplet systems are demonstrated by studying the evaporation and dissolution of ouzo drops. The fundamental fluid dynamics of the evaporating/dissolving multicomponent droplets are illuminated. We expect our work to give a contribution to bridging the gap from fluid dynamics to chemical engineering and colloidal \& interfacial science.

Much more effort is required to enable high-precision chemical engineering for droplets. Regarding to the topics discussed in this thesis, here we list some of work which might be interesting to study in the future: (i) How to locally trigger the "ouzo effect" by applying an extra field; (ii) A comprehensive theoretical model for the multiphase diffusion process at the moving liquid-liquid interface with the consideration of phase separation; (iii) The competitions of the different types of flows that driven by different forces Marangoni force, buoyant force, viscous force - inside multicomponent and multiphase droplets; (iv) Applying different properties (micro-) nanoparticles in the supraparticle fabrication by evaporating / dissolving colloidal ouzo droplets; (v) Introducing ouzo droplets into other classical droplet system, like Leidenfrost droplets, droplet impacting into liquid, micro- or picoliter ouzo droplets in micro-channel. We believe many more interesting work will be followed. 



\section{References}

[1] H. Sirringhaus, T. Kawase, R. H. Friend, T. Shimoda, M. Inbasekaran, $\mathrm{W}$. Wu, and E. P. Woo, "High-resolution inkjet printing of all-polymer transistor circuits", Science 290, 2123-2126 (2000).

[2] d. G. B.J, P. C. Duineveld, and U. Schubert, "Ink jet printing of polymers: State of the art and future developments", Adv. Materials 16, 203-213 (2004).

[3] C. Williams, "Ink-jet printers go beyond paper", Phys. World 19, 24-29 (2006).

[4] J. F. Dijksman, P. C. Duineveld, M. J. J. Hack, A. Pierik, J. M. Rensen, J. E. Rubingh, I. Schram, and M. M. Vernhout, "Precision ink jet printing of polymer light emitting displays", Journal of Materials Chemistry 17, 511-5122 (2007).

[5] N. Anton, J.-P. Benoit, and P. Saulnier, "Design and production of nanoparticles formulated from nano-emulsion templates - a review", Journal of Controlled Release 128, 185-199 (2008).

[6] C. Solans, P. Izquierdo, J. Nolla, N. Azemar, and M. Garcia-Celma, "Nano-emulsions", Current Opinion in Colloid \& Interface Science 10, 102-110 (2005).

[7] H. Hu and R. G. Larson, "Evaporation of a sessile droplet on a substrate", The Journal of Physical Chemistry B 106, 1334 (2002).

[8] B. J. Hindson, K. D. Ness, D. A. Masquelier, P. Belgrader, N. J. Heredia, A. J. Makarewicz, I. J. Bright, M. Y. Lucero, A. L. Hiddessen, T. C. Legler, et al., "High-throughput droplet digital pcr system for absolute quantitation of dna copy number", Analytical Chemistry 83, 8604-8610 (2011).

[9] E. Z. Macosko, A. Basu, R. Satija, J. Nemesh, K. Shekhar, M. Goldman, I. Tirosh, A. R. Bialas, N. Kamitaki, E. M. Martersteck, et al., "Highly parallel genome-wide expression profiling of individual cells using nanoliter droplets", Cell 161, 1202-1214 (2015).

[10] M. G. Pollack, R. B. Fair, and A. D. Shenderov, "Electrowettingbased actuation of liquid droplets for microfluidic applications", Applied Physics Letters 77, 1725-1726 (2000). 
[11] J. D. Schwarzkopf, M. Sommerfeld, C. T. Crowe, and Y. Tsuji, Multiphase flows with droplets and particles (CRC press) (2011).

[12] S. Martin and R. G. Parton, "Opinion: Lipid droplets: a unified view of a dynamic organelle", Nature Reviews Molecular Cell Biology 7, 373 (2006).

[13] N. Liu, Z. Lu, J. Zhao, M. T. McDowell, H.-W. Lee, W. Zhao, and Y. Cui, "A pomegranate-inspired nanoscale design for large-volumechange lithium battery anodes", Nature Nanotechnology 9, 187 (2014).

[14] T. M. Squires and S. R. Quake, "Microfluidics: Fluid physics at the nanoliter scale", Reviews of Modern Physics 77, 977-1026 (2005).

[15] Z. Izri, M. N. van der Linden, S. Michelin, and O. Dauchot, "Selfpropulsion of pure water droplets by spontaneous marangoni-stressdriven motion", Physical Review Letters 113, 248302 (2014).

[16] J. Davies, "Rideal. ek in: Intcrfacial phenomenon", (1963).

[17] S. Chu and A. Prosperetti, "Dissolution and growth of a multicomponent drop in an immiscible liquid", Journal of Fluid Mechanics 798, 787-811 (2016).

[18] J. Crank, The mathematics of diffusion (Oxford university press) (1979).

[19] D. Quéré, "Wetting and roughness", Annual Review of Materials Research 38, 71-99 (2008).

[20] D. Bonn, J. Eggers, J. Indekeu, J. Meunier, and E. Rolley, "Wetting and spreading", Reviews of Modern Physics 81, 739-805 (2009).

[21] M. Rein, "Phenomena of liquid drop impact on solid and liquid surfaces", Fluid Dynamics Research 12, 61-93 (1993).

[22] A. L. Yarin, "Drop impact dynamics: splashing, spreading, receding, bouncing. ..", Annual Review of Fluid Mechanics 38, 159-192 (2006).

[23] R. Enright, N. Miljkovic, J. L. Alvarado, K. Kim, and J. W. Rose, "Dropwise condensation on micro-and nanostructured surfaces", Nanoscale and Microscale Thermophysical Engineering 18, 223-250 (2014).

[24] M. Geri, B. Keshavarz, G. H. McKinley, and J. W. Bush, "Thermal delay of drop coalescence", Journal of Fluid Mechanics 833 (2017).

[25] H. A. Stone, A. D. Stroock, and A. Ajdari, "Engineering flows in small devices: microfluidics toward a lab-on-a-chip", Annual Review of Fluid Mechanics 36, 381-411 (2004).

[26] S. L. Anna, N. Bontoux, and H. A. Stone, "Formation of dispersions using "flow focusing" in microchannels", Applied physics letters 82, 364366 (2003).

[27] M. Shirota, M. A. van Limbeek, C. Sun, A. Prosperetti, and D. Lohse, "Dynamic leidenfrost effect: relevant time and length scales", Physical Review Letters 116, 064501 (2016). 
[28] J. L. Anderson and D. C. Prieve, "Diffusiophoresis: migration of colloidal particles in gradients of solute concentration", Separation and Purification Methods 13, 67-103 (1984).

[29] N. Shi, R. Nery-Azevedo, A. I. Abdel-Fattah, and T. M. Squires, "Diffusiophoretic focusing of suspended colloids", Physical Review Letters 117, 258001 (2016).

[30] M. Z. Bazant and T. M. Squires, "Induced-charge electrokinetic phenomena: theory and microfluidic applications", Physical Review Letters 92, 066101 (2004).

[31] T. M. Squires and M. Z. Bazant, "Induced-charge electro-osmosis", Journal of Fluid Mechanics 509, 217-252 (2004).

[32] Y. O. Popov, "Evaporative deposition patterns: Spatial dimensions of the deposit", Physical Review E 71, 036313 (2005).

[33] H. Gelderblom, A. G. Marin, H. Nair, A. van Houselt, L. Lefferts, J. H. Snoeijer, and D. Lohse, "How water droplets evaporate on a superhydrophobic substrate", Physical Review E 83, 026306 (2011).

[34] S. Dash and S. V. Garimella, "Droplet evaporation dynamics on a superhydrophobic surface with negligible hysteresis", Langmuir 29, 1078510795 (2013).

[35] A.-M. Cazabat and G. Guéna, "Evaporation of macroscopic sessile droplets", Soft Matter 6, 2591-2612 (2010).

[36] X. Zhang, J. Wang, L. Bao, E. Dietrich, R. C. A. van der Veen, S. Peng, J. Friend, H. J. W. Zandvliet, L. Yeo, and D. Lohse, "Mixed mode of dissolving immersed microdroplets at a solid-water interface", Soft Matter 11, 1889-1900 (2015).

[37] D. Lohse and X. Zhang, "Surface nanobubbles and nanodroplets", Reviews of Modern Physics 87, 981-1035 (2015).

[38] R. D. Deegan, O. Bakajin, T. F. Dupont, G. Huber, S. R. Nagel, and T. A. Witten, "Capillary flow as the cause of ring stains from dried liquid drops", Nature 389, 827-829 (1997).

[39] R. D. Deegan, O. Bakajin, T. F. Dupont, G. Huber, S. R. Nagel, and T. A. Witten, "Contact line deposits in an evaporating drop", Physical Review E 62, 756-765 (2000).

[40] H. Y. Erbil, "Evaporation of pure liquid sessile and spherical suspended drops: A review", Advances in Colloid and Interface Science 170, 67-86 (2012).

[41] S. A. Vitale and J. L. Katz, "Liquid droplet dispersions formed by homogeneous liquid- liquid nucleation:"the ouzo effect"”, Langmuir 19, 4105-4110 (2003).

[42] F. Ganachaud and J. L. Katz, "Nanoparticles and nanocapsules created 
using the ouzo effect: Spontaneous emulsification as an alternative to ultrasonic and high-shear devices", ChemPhysChem 6, 209-216 (2005).

[43] R. Botet, "The "ouzo effect", recent developments and application to therapeutic drug carrying", Journal of Physics: Conference Series 352, 012047 (2012).

[44] D. Guzey and D. J. McClements, "Formation, stability and properties of multilayer emulsions for application in the food industry", Advances in Colloid and Interface Science 128, 227-248 (2006).

[45] J. Gutiérrez, C. González, A. Maestro, I. Solè, C. Pey, and J. Nolla, "Nano-emulsions: New applications and optimization of their preparation", Current Opinion in Colloid \& Interface Science 13, 245-251 (2008).

[46] A. Jain and K. K. Verma, "Recent advances in applications of singledrop microextraction: A review", Analytica Chimica Acta 706, 37 - 65 (2011).

[47] J. T. Davies, E. K. Rideal, and M. Bender, "Interfacial phenomena", Journal of The Electrochemical Society 109, 175C-175C (1962).

[48] N. L. Sitnikova, R. Sprik, G. Wegdam, and E. Eiser, "Spontaneously formed trans-anethol/water/alcohol emulsions: mechanism of formation and stability", Langmuir 21, 7083-7089 (2005).

[49] T. Ahmed, Equations of state and PVT analysis (Elsevier) (2013).

[50] R. Picknett and R. Bexon, "The evaporation of sessile or pendant drops in still air", Journal of Colloid and Interface Science 61, 336-350 (1977).

[51] K. Sefiane, L. Tadrist, and M. Douglas, "Experimental study of evaporating water-ethanol mixture sessile drop: influence of concentration", International Journal of Heat and Mass Transfer 46, 4527-4534 (2003).

[52] N. Shahidzadeh-Bonn, S. Rafai, A. Azouni, and D. Bonn, "Evaporating droplets", Journal of Fluid Mechanics 549, 307-313 (2006).

[53] W. D. Ristenpart, P. G. Kim, C. Domingues, J. Wan, and H. A. Stone, "Influence of substrate conductivity on circulation reversal in evaporating drops", Physical Review Letters 99, 234502 (2007).

[54] J. A. Lim, W. H. Lee, H. S. Lee, J. H. Lee, Y. D. Park, and K. Cho, "Self-organization of ink-jet-printed triisopropylsilylethynyl pentacene via evaporation-induced flows in a drying droplet", Advanced Functional Materials 18, 229-234 (2008).

[55] T. Ming, X. Kou, H. Chen, T. Wang, H.-L. Tam, K.-W. Cheah, J.-Y. Chen, and J. Wang, "Ordered gold nanostructure assemblies formed by droplet evaporation", Angewandte Chemie 120, 9831-9836 (2008).

[56] K. Sefiane, S. David, and M. E. R. Shanahan, "Wetting and evaporation of binary mixture drops", The Journal of Physical Chemistry B 112, 11317-11323 (2008). 
[57] C. Liu, E. Bonaccurso, and H.-J. Butt, "Evaporation of sessile water/ethanol drops in a controlled environment", Physical Chemistry Chemical Physics 10, 7150-7157 (2008).

[58] F. Schönfeld, K. H. Graf, S. Hardt, and H.-J. Butt, "Evaporation dynamics of sessile liquid drops in still air with constant contact radius", International Journal of Heat and Mass Transfer 51, 3696-3699 (2008).

[59] J. R. E. Christy, Y. Hamamoto, and K. Sefiane, "Flow transition within an evaporating binary mixture sessile drop", Physical Review Letters 106, 205701 (2011).

[60] A. G. Marin, H. Gelderblom, D. Lohse, and J. H. Snoeijer, "Orderto-disorder transition in ring-shaped colloidal stains", Physical Review Letters 107, 085502 (2011).

[61] D. Brutin, B. Sobac, B. Loquet, and J. Sampol, "Pattern formation in drying drops of blood", Journal of Fluid Mechanics 667, 85-95 (2011).

[62] R. Ledesma-Aguilar, D. Vella, and J. M. Yeomans, "Lattice-boltzmann simulations of droplet evaporation", Soft Matter 10, 8267-8275 (2014).

[63] X. Zhang and W. Ducker, "Formation of interfacial nanodroplets through changes in solvent quality", Langmuir 23, 12478-12480 (2007).

[64] D. R. Lide, CRC handbook of chemistry and physics (CRC press) (2004).

[65] S. Torza and S. Mason, "Three-phase interactions in shear and electrical fields", Journal of Colloid and Interface Science 33, 67-83 (1970).

[66] I. B. Bazhlekov and P. J. Shopov, "Numerical simulation of dynamic contact-line problems", Journal of Fluid Mechanics 352, 113-133 (1997).

[67] B. González, N. Calvar, E. Gómez, and Á. Domínguez, "Density, dynamic viscosity, and derived properties of binary mixtures of methanol or ethanol with water, ethyl acetate, and methyl acetate at $\mathrm{t}=(293.15$, 298.15, and 303.15) k", The Journal of Chemical Thermodynamics 39, 1578-1588 (2007).

[68] G. Vazquez, E. Alvarez, and J. M. Navaza, "Surface tension of alcohol water + water from 20 to 50 .degree.C", Journal of Chemical \& Engineering Data 40, 611-614 (1995).

[69] S. Pařez, G. Guevara-Carrion, H. Hasse, and J. Vrabec, "Mutual diffusion in the ternary mixture of water + methanol + ethanol and its binary subsystems", Physical Chemistry Chemical Physics 15, 3985 (2013).

[70] A. Zuend, C. Marcolli, B. P. Luo, and T. Peter, "A thermodynamic model of mixed organic-inorganic aerosols to predict activity coefficients", Atmospheric Chemistry and Physics 8, 4559-4593 (2008).

[71] A. Zuend, C. Marcolli, A. M. Booth, D. M. Lienhard, V. Soonsin, U. K. Krieger, D. O. Topping, G. McFiggans, T. Peter, and J. H. Seinfeld, "New and extended parameterization of the thermodynamic model 
AIOMFAC: calculation of activity coefficients for organic-inorganic mixtures containing carboxyl, hydroxyl, carbonyl, ether, ester, alkenyl, alkyl, and aromatic functional groups", Atmospheric Chemistry and Physics 11, 9155-9206 (2011).

[72] H. Kim, F. m. c. Boulogne, E. Um, I. Jacobi, E. Button, and H. A. Stone, "Controlled uniform coating from the interplay of marangoni flows and surface-adsorbed macromolecules", Physical Review Letters 116, 124501 (2016).

[73] X. H. Zhang, J. M. Ren, H. J. Yang, Y. H. He, J. F. Tan, and G. G. Qiao, "From transient nanodroplets to permanent nanolenses", Soft Matter 8, 4314-4317 (2012).

[74] T. R. Hughes, M. Mao, A. R. Jones, J. Burchard, M. J. Marton, K. W. Shannon, S. M. Lefkowitz, M. Ziman, J. M. Schelter, M. R. Meyer, et al., "Expression profiling using microarrays fabricated by an ink-jet oligonucleotide synthesizer", Nature Biotechnology 19, 342-347 (2001).

[75] B. Creran, X. Li, B. Duncan, C. S. Kim, D. F. Moyano, and V. M. Rotello, "Detection of bacteria using inkjet-printed enzymatic test strips", ACS Applied Materials \& Interfaces 6, 19525-19530 (2014).

[76] S. V. Murphy and A. Atala, "3d bioprinting of tissues and organs", Nature Biotechnology 32, 773-785 (2014).

[77] L. L. da Luz, R. Milani, J. F. Felix, I. R. B. Ribeiro, M. Talhavini, B. A. D. Neto, J. Chojnacki, M. O. Rodrigues, and S. A. Júnior, "Inkjet printing of lanthanide-organic frameworks for anti-counterfeiting applications", ACS Applied Materials \& Interfaces 7, 27115-27123 (2015).

[78] K. Yamada, T. G. Henares, K. Suzuki, and D. Citterio, "Paper-based inkjet-printed microfluidic analytical devices", Angewandte Chemie International Edition 54, 5294-5310 (2015).

[79] E. P. Kateri, S. W. William, E. R. Steven, and A. S. Robert, "Additive jet printing of polymer thin-film transistors", Applied Physics Letters 83, 2070 (2003).

[80] L. Bao, A. r. Rezk, L. Y. Yeo, and X. Zhang, "Highly ordered arrays of femtoliter surface droplets", Small 11, 4850-4855 (2015).

[81] J. C. Maxwell, ERRATA. VOL. II., volume 2 of Cambridge Library Collection - Physical Sciences, viii-viii (Cambridge University Press) (2011).

[82] S. Semenov, A. Trybala, H. Agogo, N. Kovalchuk, F. Ortega, R. G. Rubio, V. M. Starov, and M. G. Velarde, "Evaporation of droplets of surfactant solutions", Langmuir 29, 10028-10036 (2013).

[83] P. Calvert, "Inkjet printing for materials and devices", Chemistry of Materials 13, 3299-3305 (2001). 
[84] J. Park and J. Moon, "Control of colloidal particle deposit patterns within picoliter droplets ejected by ink-jet printing", Langmuir 22, 35063513 (2006).

[85] S. Kim, M. Misner, T. Xu, M. Kimura, and T. Russell, "Highly oriented and ordered arrays from block copolymers via solvent evaporation", Advanced Materials 16, 226-231 (2004).

[86] M. Schena, D. Shalon, R. W. Davis, and P. O. Brown, "Quantitative monitoring of gene expression patterns with a complementary dna microarray", Science 270, 467-470 (1995).

[87] D. Bensimon, A. J. Simon, V. Croquette, and A. Bensimon, "Stretching dna with a receding meniscus: Experiments and models", Physical Review Letters 74, 4754-4757 (1995).

[88] H. Y. Erbil, "Evaporation of pure liquid sessile and spherical suspended drops: A review", Advances in Colloid and Interface Science 170, 67 86 (2012).

[89] D. Brutin, Droplet wetting and evaporation: from pure to complex fluids (Academic Press) (2015).

[90] K. Birdi and D. Vu, "Wettability and the evaporation rates of fluids from solid surfaces", Journal of Adhesion Science and Technology 7, 485-493 (1993).

[91] S. Kulinich and M. Farzaneh, "Effect of contact angle hysteresis on water droplet evaporation from super-hydrophobic surfaces", Applied Surface Science 255, 4056 - 4060 (2009).

[92] N. Anantharaju, M. Panchagnula, and S. Neti, "Evaporating drops on patterned surfaces: Transition from pinned to moving triple line", Journal of Colloid and Interface Science 337, 176 - 182 (2009).

[93] E. Dietrich, E. S. Kooij, X. Zhang, H. J. W. Zandvliet, and D. Lohse, "Stick-jump mode in surface droplet dissolution", Langmuir 31, 46964703 (2015).

[94] H. Hu and R. G. Larson, "Analysis of the effects of Marangoni stresses on the microflow in an evaporating sessile droplet", Langmuir 21, 39723980 (2005).

[95] Z. Pan, S. Dash, J. A. Weibel, and S. V. Garimella, "Assessment of water droplet evaporation mechanisms on hydrophobic and superhydrophobic substrates", Langmuir 29, 15831-15841 (2013).

[96] P. J. Yunker, T. Still, M. A. Lohr, and A. G. Yodh, "Suppression of the coffee-ring effect by shape-dependent capillary interactions", Nature 476, 308-311 (2011).

[97] T. Still, P. J. Yunker, and A. G. Yodh, "Surfactant-induced marangoni eddies alter the coffee-rings of evaporating colloidal drops", Langmuir 
28, 4984-4988 (2012).

[98] W. Sempels, R. De Dier, H. Mizuno, J. Hofkens, and J. Vermant, "Autoproduction of biosurfactants reverses the coffee ring effect in a bacterial system", Nature Communications 4, 1757 EP - (2013).

[99] A. Marin, R. Liepelt, M. Rossi, and C. J. Kahler, "Surfactant-driven flow transitions in evaporating droplets", Soft Matter 12, 1593-1600 (2016).

[100] R. Bennacer and K. Sefiane, "Vortices, dissipation and flow transition in volatile binary drops", Journal of Fluid Mechanics 749, 649-665 (2014).

[101] H. Tan, C. Diddens, P. Lv, J. G. M. Kuerten, X. Zhang, and D. Lohse, "Evaporation-triggered microdroplet nucleation and the four life phases of an evaporating ouzo drop", Proceedings of the National Academy of Sciences 113, 8642-8647 (2016).

[102] J. M. Stauber, S. K. Wilson, B. R. Duffy, and K. Sefiane, "Evaporation of droplets on strongly hydrophobic substrates", Langmuir 31, 3653-3660 (2015).

[103] X. Deng, L. Mammen, H.-J. Butt, and D. Vollmer, "Candle soot as a template for a transparent robust superamphiphobic coating", Science 335, 67-70 (2012).

[104] M. Paven, P. Papadopoulos, L. Mammen, X. Deng, H. Sachdev, D. Vollmer, and H.-J. Butt, "Optimization of superamphiphobic layers based on candle soot", Pure and Applied Chemistry 86, 87-96 (2014).

[105] Y. Song, Z. Lu, H. Yang, S. Zhang, and X. Zhang, "Dissolution of sessile microdroplets of electrolyte and graphene oxide solutions in an ouzo system", Langmuir 32, 10296-10304 (2016).

[106] J. D. Smith, R. Dhiman, S. Anand, E. Reza-Garduno, R. E. Cohen, G. H. McKinley, and K. K. Varanasi, "Droplet mobility on lubricantimpregnated surfaces", Soft Matter 9, 1772-1780 (2013).

[107] F. Schellenberger, J. Xie, N. Encinas, A. Hardy, M. Klapper, P. Papadopoulos, H.-J. Butt, and D. Vollmer, "Direct observation of drops on slippery lubricant-infused surfaces", Soft Matter 11, 7617-7626 (2015).

[108] P.-G. De Gennes, F. Brochard-Wyart, and D. Quéré, Capillarity and wetting phenomena: drops, bubbles, pearls, waves (Springer New York) (2004).

[109] G. M. Kontogeorgis and S. Kiil, Introduction to applied colloid and surface chemistry (John Wiley \& Sons) (2016).

[110] C. Diddens, J. G. M. Kuerten, C. W. M. van der Geld, and H. M. A. Wijshoff, "Modeling the evaporation of sessile multi-component droplets", Journal of Colloid and Interface Science 487, 426-436 (2017).

[111] C. Diddens, "Detailed finite element method modeling of evaporating multi-component droplets", Journal of Computational Physics 340, 670- 
687 (2017).

[112] C. Diddens, H. Tan, P. Lv, M. Versluis, J. Kuerten, X. Zhang, and D. Lohse, "Evaporating pure, binary and ternary droplets: thermal effects and axial symmetry breaking", Journal of Fluid Mechanics 823, 470-497 (2017).

[113] R. Wittig, J. Lohmann, and J. Gmehling, "Vapor-liquid equilibria by unifac group contribution. 6. revision and extension", Industrial \& Engineering Chemistry Research 42, 183-188 (2003).

[114] M. Lapuerta, J. P. Hernández, and J. R. Agudelo, "An equation for the estimation of alcohol-air diffusion coefficients for modelling evaporation losses in fuel systems", Applied Thermal Engineering 73, 539 - 548 (2014).

[115] F. Girard, M. Antoni, S. Faure, and A. Steinchen, "Influence of heating temperature and relative humidity in the evaporation of pinned droplets", Colloids and Surfaces A: Physicochemical and Engineering Aspects 323, 36 - 49 (2008).

[116] S. Dash and S. V. Garimella, "Droplet evaporation on heated hydrophobic and superhydrophobic surfaces", Physical Review E 89, 042402 (2014).

[117] K. Gleason and S. A. Putnam, "Microdroplet evaporation with a forced pinned contact line", Langmuir 30, 10548-10555 (2014).

[118] M.-R. Kula, K. H. Kroner, and H. Hustedt, Purification of enzymes by liquid-liquid extraction, 73-118 (Springer Berlin Heidelberg, Berlin, Heidelberg) (1982).

[119] K. Fukumoto, M. Yoshizawa, and H. Ohno, "Room temperature ionic liquids from 20 natural amino acids", Journal of the American Chemical Society 127, 2398-2399 (2005).

[120] P. Chasanis, M. Brass, and E. Y. Kenig, "Investigation of multicomponent mass transfer in liquid-liquid extraction systems at microscale", International Journal of Heat and Mass Transfer 53, 3758-3763 (2010).

[121] S. Ahuja, ed., Handbook of Bioseparations, Volume 2 (Academic press) (2000).

[122] D. Lohse, "Towards controlled liquid-liquid microextraction", Journal of Fluid Mechanics 804, 1-4 (2016).

[123] P. S. Epstein and M. S. Plesset, "On the stability of gas bubbles in liquid-gas solutions", The Journal of Chemical Physics 18, 1505-1509 (1950).

[124] P. B. Duncan and D. Needham, "Microdroplet dissolution into a secondphase solvent using a micropipet technique: Test of the epstein- plesset model for an aniline- water system", Langmuir 22, 4190-4197 (2006). 
[125] J. T. Su and D. Needham, "Mass transfer in the dissolution of a multicomponent liquid droplet in an immiscible liquid environment", Langmuir 29, 13339-13345 (2013).

[126] S. Maheshwari, M. van der Hoef, A. Prosperetti, and D. Lohse, "Molecular dynamics study of multicomponent droplet dissolution in a sparingly miscible liquid", Journal of Fluid Mechanics 833, 54-69 (2017).

[127] E. Dietrich, S. Wildeman, C. W. Visser, K. Hofhuis, E. S. Kooij, H. J. W. Zandvliet, and D. Lohse, "Role of natural convection in the dissolution of sessile droplets", Journal of Fluid Mechanics 794, 45-67 (2016).

[128] E. Dietrich, M. Rump, P. Lv, E. S. Kooij, H. J. Zandvliet, and D. Lohse, "Segregation in dissolving binary-component sessile droplets", Journal of Fluid Mechanics 812, 349-369 (2017).

[129] C. Solans, D. Morales, and M. Homs, "Spontaneous emulsification", Current Opinion in Colloid \& Interface Science 22, 88-93 (2016).

[130] K. J. Ruschak and C. A. Miller, "Spontaneous emulsification in ternary systems with mass transfer", Industrial \& Engineering Chemistry Fundamentals 11, 534-540 (1972).

[131] J. Canny, "A computational approach to edge detection", IEEE Transactions on Pattern Analysis and Machine Intelligence PAMI-8, 679-698 (1986).

[132] W. Thielicke and E. Stamhuis, "Pivlab-towards user-friendly, affordable and accurate digital particle image velocimetry in matlab", Journal of Open Research Software 2, e30 (2014).

[133] W. Thielicke, "The flapping flight of birds: Analysis and application", Ph.D. thesis, Rijksuniversiteit Groningen (2014).

[134] V. Mathai, E. Calzavarini, J. Brons, C. Sun, and D. Lohse, "Microbubbles and microparticles are not faithful tracers of turbulent acceleration", Physical Review Letters 117, 024501 (2016).

[135] J. Oliveira, C. van der Geld, and J. Kuerten, "Lagrangian velocity and acceleration statistics of fluid and inertial particles measured in pipe flow with 3d particle tracking velocimetry", International Journal of Multiphase Flow 73, 97 - 107 (2015).

[136] H. Tan, C. Diddens, M. Versluis, H.-J. Butt, D. Lohse, and X. Zhang, "Self-wrapping of an ouzo drop induced by evaporation on a superamphiphobic surface", Soft Matter 13, 2749-2759 (2017).

[137] G. S. Hartley, "Diffusion and swelling of high polymers. part i.-the swelling and solution of a high polymer solid considered as a diffusion process", Transactions of the Faraday Society 42, B006-B011 (1946).

[138] J. S. Kirkaldy and L. Brown, "Diffusion behaviour in ternary, multiphase systems", Canadian Metallurgical Quarterly 2, 89-115 (1963). 
[139] S. I. Barry and J. Caunce, "Exact and numerical solutions to a stefan problem with two moving boundaries", Applied Mathematical Modelling 32, 83 - 98 (2008).

[140] J. Crank, Free And Moving Boundary Problems (Oxford Science Publications) (Oxford University Press New York) (1987).

[141] A. Fredenslund, R. L. Jones, and J. M. Prausnitz, "Group-contribution estimation of activity coefficients in nonideal liquid mixtures", AIChE Journal 21, 1086-1099 (1975).

[142] A. Fredenslund, J. Gmehling, and P. Rasmussen, eds., Vapor-liquid Equilibria Using Unifac, volume Fredenslund (Elsevier) (1977).

[143] D. Constantinescu and J. Gmehling, "Further development of modified unifac (dortmund): Revision and extension 6", Journal of Chemical \& Engineering Data 61, 2738-2748 (2016).

[144] A. Zuend, C. Marcolli, T. Peter, and J. H. Seinfeld, "Computation of liquid-liquid equilibria and phase stabilities: implications for rhdependent gas/particle partitioning of organic-inorganic aerosols", Atmospheric Chemistry and Physics 10, 7795-7820 (2010).

[145] L. Perkins and C. Geankoplis, "Molecular diffusion in a ternary liquid system with the diffusing component dilute", Chemical Engineering Science 24, 1035 - 1042 (1969).

[146] W. Hayduk and B. S. Minhas, "Correlations for prediction of molecular diffusivities in liquids", The Canadian Journal of Chemical Engineering 60, 295-299 (1982).

[147] D. Tam, V. von ARNIM, G. McKinley, and A. Hosoi, "Marangoni convection in droplets on superhydrophobic surfaces", Journal of Fluid Mechanics 624, 101-123 (2009).

[148] S. M. Rowan, M. I. Newton, F. W. Driewer, and G. McHale, "Evaporation of microdroplets of azeotropic liquids", The Journal of Physical Chemistry B 104, 8217-8220 (2000).

[149] A. K. H. Cheng, D. M. Soolaman, and H.-Z. Yu, "Evaporation of microdroplets of ethanol-water mixtures on gold surfaces modified with self-assembled monolayers", The Journal of Physical Chemistry B 110, 11267-11271 (2006).

[150] Z. Wang, X.-F. Peng, A. S. Mujumdar, A. Su, and D.-J. Lee, "Evaporation of ethanol-water mixture drop on horizontal substrate", Drying Technology 26, 806-810 (2008).

[151] L. Shi, P. Shen, D. Zhang, Q. Lin, and Q. Jiang, "Wetting and evaporation behaviors of water-ethanol sessile drops on PTFE surfaces", Surface and Interface Analysis 41, 951-955 (2009).

[152] P. Innocenzi, L. Malfatti, S. Costacurta, T. Kidchob, M. Piccinini, and 
A. Marcelli, "Evaporation of ethanol and ethanol-water mixtures studied by time-resolved infrared spectroscopy", The Journal of Physical Chemistry A 112, 6512-6516 (2008).

[153] X. Zhong and F. Duan, "Flow regime and deposition pattern of evaporating binary mixture droplet suspended with particles", The European Physical Journal E 39 (2016).

[154] N. J. Cira, A. Benusiglio, and M. Prakash, "Vapour-mediated sensing and motility in two-component droplets", Nature 519, 446-450 (2015).

[155] J.-P. E. Grolier and E. Wilhelm, "Excess volumes and excess heat capacities of water + ethanol at 298.15 K", Fluid Phase Equilibria 6, 283-287 (1981).

[156] R. Yano, Y. Fukuda, and T. Hashi, "Thermal conductivity measurement of water-ethanol solutions by the laser-induced transient grating method", Chem. Phys. 124, 315-319 (1988).

[157] A. Logg, Automated solution of differential equations by the finite element method the FEniCS book (Springer, Berlin New York) (2012).

[158] H. Machrafi, A. Rednikov, P. Colinet, and P. Dauby, "Bénard instabilities in a binary-liquid layer evaporating into an inert gas", Journal of Colloid and Interface Science 349, 331-353 (2010).

[159] V. M. Rodrigues, P. T. Rosa, M. O. Marques, A. J. Petenate, and M. A. A. Meireles, "Supercritical extraction of essential oil from aniseed (pimpinella anisum l) using co2: solubility, kinetics, and composition data", Journal of Agricultural and Food Chemistry 51, 1518-1523 (2003).

[160] I. Langmuir and V. J. Schaefer, "Rates of evaporation of water through compressed monolayers on water", Journal of the Franklin Institute 235, 119-162 (1943).

[161] P. E. Farrell, M. D. Piggott, C. C. Pain, G. J. Gorman, and C. R. Wilson, "Conservative interpolation between unstructured meshes via supermesh construction", Computer Methods in Applied Mechanics and Engineering 198, 2632-2642 (2009).

[162] B. Sobac and D. Brutin, "Thermocapillary instabilities in an evaporating drop deposited onto a heated substrate", Physics of Fluids 24, 032103 (2012).

[163] V. Rastogi, S. Melle, O. G. Calderon, A. A. García, M. Marquez, and O. D. Velev, "Synthesis of light-diffracting assemblies from microspheres and nanoparticles in droplets on a superhydrophobic surface", Advanced Materials 20, 4263-4268 (2008).

[164] M. Sperling, P. Papadopoulos, and M. Gradzielski, "Understanding the formation of anisometric supraparticles: A mechanistic look inside 
droplets drying on a superhydrophobic surface", Langmuir 32, 69026908 (2016).

[165] V. Rastogi, A. A. García, M. Marquez, and O. D. Velev, "Anisotropic particle synthesis inside droplet templates on superhydrophobic surfaces", Macromolecular Rapid Communications 31, 190-195 (2010).

[166] M. Sperling, V. J. Spiering, O. D. Velev, and M. Gradzielski, "Controlled formation of patchy anisometric fumed silica supraparticles in droplets on bent superhydrophobic surfaces", Particle \& Particle Systems Characterization $\mathbf{3 4}$ (2017).

[167] S. Wooh, H. Huesmann, M. N. Tahir, M. Paven, K. Wichmann, D. Vollmer, W. Tremel, P. Papadopoulos, and H.-J. Butt, "Synthesis of mesoporous supraparticles on superamphiphobic surfaces", Advanced Materials 27, 7338-7343 (2015).

[168] M. B. Gawande, A. Goswami, T. Asefa, H. Guo, A. V. Biradar, D.-L. Peng, R. Zboril, and R. S. Varma, "Core-shell nanoparticles: synthesis and applications in catalysis and electrocatalysis", Chemical Society Reviews 44, 7540-7590 (2015).

[169] M. Sperling, H.-J. Kim, O. D. Velev, and M. Gradzielski, "Active steerable catalytic supraparticles shuttling on preprogrammed vertical trajectories", Advanced Materials Interfaces 3, 1600095 (2016).

[170] D.-W. Lee, M.-H. Jin, C.-B. Lee, D. Oh, S.-K. Ryi, J.-S. Park, J.-S. Bae, Y.-J. Lee, S.-J. Park, and Y.-C. Choi, "Facile synthesis of mesoporous silica and titania supraparticles by a meniscus templating route on a superhydrophobic surface and their application to adsorbents", Nanoscale 6, 3483-3487 (2014).

[171] Z. Wu and D. Zhao, "Ordered mesoporous materials as adsorbents", Chemical Communications 47, 3332-3338 (2011).

[172] A. Choi, K. D. Seo, B. C. Kim, D. S. Kim, et al., "Recent advances in engineering microparticles and their nascent utilization in biomedical delivery and diagnostic applications", Lab on a Chip 17, 591-613 (2017).

[173] K. Unger, D. Kumar, M. Grün, G. Büchel, S. Lüdtke, T. Adam, K. Schumacher, and S. Renker, "Synthesis of spherical porous silicas in the micron and submicron size range: challenges and opportunities for miniaturized high-resolution chromatographic and electrokinetic separations", Journal of Chromatography A 892, 47-55 (2000).

[174] J. F. Galisteo-López, M. Ibisate, R. Sapienza, L. S. Froufe-Pérez, Á. Blanco, and C. López, "Self-assembled photonic structures", Advanced Materials 23, 30-69 (2011).

[175] Y. Zhao, Y. Cheng, L. Shang, J. Wang, Z. Xie, and Z. Gu, "Microfluidic synthesis of barcode particles for multiplex assays", Small 11, 151-174 
(2015)

[176] K. Burkert, T. Neumann, J. Wang, U. Jonas, W. Knoll, and H. Ottleben, "Automated preparation method for colloidal crystal arrays of monodisperse and binary colloid mixtures by contact printing with a pintool plotter", Langmuir 23, 3478-3484 (2007).

[177] V. Rastogi and O. D. Velev, "Development and evaluation of realistic microbioassays in freely suspended droplets on a chip", Biomicrofluidics 1, 014107 (2007).

[178] J. Zhou, J. Yang, Z. Gu, G. Zhang, Y. Wei, X. Yao, Y. Song, and L. Jiang, "Controllable fabrication of noniridescent microshaped photonic crystal assemblies by dynamic three-phase contact line behaviors on superhydrophobic substrates", ACS applied materials \& interfaces 7, 22644-22651 (2015).

[179] H. Eral, J. Oh, et al., "Contact angle hysteresis: a review of fundamentals and applications", Colloid and Polymer Science 291, 247-260 (2013).

[180] J. R. Millman, K. H. Bhatt, B. G. Prevo, and O. D. Velev, "Anisotropic particle synthesis in dielectrophoretically controlled microdroplet reactors", Nature Materials 4, 98 (2005).

[181] T. Sekido, S. Wooh, R. Fuchs, M. Kappl, Y. Nakamura, H.-J. Butt, and S. Fujii, "Controlling the structure of supraballs by ph-responsive particle assembly", Langmuir 33, 1995-2002 (2017).

[182] M. Sperling, O. D. Velev, and M. Gradzielski, "Controlling the shape of evaporating droplets by ionic strength: formation of highly anisometric silica supraparticles", Angewandte Chemie International Edition 53, 586-590 (2014).

[183] Á. G. Marín, H. Gelderblom, A. Susarrey-Arce, A. van Houselt, L. Lefferts, J. G. Gardeniers, D. Lohse, and J. H. Snoeijer, "Building microscopic soccer balls with evaporating colloidal fakir drops", Proceedings of the National Academy of Sciences 109, 16455-16458 (2012).

[184] P. Li, Y. Li, Z.-K. Zhou, S. Tang, X.-F. Yu, S. Xiao, Z. Wu, Q. Xiao, Y. Zhao, H. Wang, et al., "Evaporative self-assembly of gold nanorods into macroscopic 3d plasmonic superlattice arrays", Advanced Materials 28, 2511-2517 (2016).

[185] Z. Lu, A. Rezk, F. Jativa, L. Yeo, and X. Zhang, "Dissolution dynamics of a suspension droplet in a binary solution for controlled nanoparticle assembly", Nanoscale 9, 13441-13448 (2017).

[186] M. Grzelczak, J. Vermant, E. M. Furst, and L. M. Liz-Marzán, "Directed self-assembly of nanoparticles", ACS Nano 4, 3591-3605 (2010).

[187] J. Zhou, X. Man, Y. Jiang, et al., "Structure formation in soft-matter so- 
lutions induced by solvent evaporation", Advanced Materials 29 (2017). [188] N. Vogel, S. Utech, G. T. England, T. Shirman, K. R. Phillips, N. Koay, I. B. Burgess, M. Kolle, D. A. Weitz, and J. Aizenberg, "Color from hierarchy: Diverse optical properties of micron-sized spherical colloidal assemblies", Proceedings of the National Academy of Sciences 112, 1084510850 (2015).

[189] S. Karpitschka, A. Pandey, L. A. Lubbers, J. H. Weijs, L. Botto, S. Das, B. Andreotti, and J. H. Snoeijer, "Liquid drops attract or repel by the inverted cheerios effect", Proceedings of the National Academy of Sciences 113, 7403-7407 (2016).

[190] D. Mampallil and H. B. Eral, "A review on suppression and utilization of the coffee-ring effect", Advances in Colloid and Interface Science (2018).

[191] E. Koos and N. Willenbacher, "Capillary forces in suspension rheology", Science 331, 897-900 (2011).

[192] M. T. Gokmen and F. E. Du Prez, "Porous polymer particles - a comprehensive guide to synthesis, characterization, functionalization and applications", Progress in Polymer Science 37, 365-405 (2012).

[193] S. A. Vitale and J. L. Katz, "Liquid droplet dispersions formed by homogeneous liquid- liquid nucleation:"the ouzo effect"", Langmuir 19, 4105-4110 (2003).

[194] A. Mendez-Vilas, A. B. Jodar-Reyes, and M. L. Gonzalez-Martin, "Ultrasmall Liquid Droplets on Solid Surfaces: Production, Imaging, and Relevance for Current Wetting Research", Small 5, 1366-1390 (2009).

[195] D. T. Chiu and R. M. Lorenz, "Chemistry and biology in femtoliter and picoliter volume droplets", Accounts of Chemical Research 42, 649-658 (2009).

[196] J. Shemesh, T. Ben Arye, J. Avesar, J. H. Kang, A. Fine, M. Super, A. Meller, D. E. Ingber, and S. Levenberg, "Stationary nanoliter droplet array with a substrate of choice for single adherent/nonadherent cell incubation and analysis", Proceedings of the National Academy of Sciences 111, 11293-11298 (2014).

[197] R. U. Meckenstock, F. von Netzer, C. Stumpp, T. Lueders, A. M. Himmelberg, N. Hertkorn, P. Schmitt-Kopplin, M. Harir, R. Hosein, S. Haque, and D. Schulze-Makuch, "Water droplets in oil are microhabitats for microbial life", Science 345, 673-676 (2014).

[198] P. Day, A. Manz, and Y. Zhang, Microdroplet technology principles and emerging applications in biology and chemistry (Integrated analytical systems) (NY: Springer, New York) (2012).

[199] S.-T. Lou, Z.-Q. Ouyang, Y. Zhang, X.-J. Li, J. Hu, M.-Q. Li, and F.-J. Yang, "Nanobubbles on solid surface imaged by atomic force mi- 
croscopy", Journal of Vacuum Science \& Technology B: Microelectronics and Nanometer Structures Processing, Measurement, and Phenomena 18, 2573-2575 (2000).

[200] V. Belova, M. Krasowska, D. Wang, J. Ralston, D. G. Shchukin, and H. Moehwald, "Influence of adsorbed gas at liquid/solid interfaces on heterogeneous cavitation", Chem. Sci. 4, 248-256 (2013).

[201] V. S. J. Craig, "Very small bubbles at surfaces - the nanobubble puzzle", Soft Matter 7, 40-48 (2011).

[202] H. Yang, S. Peng, X. Hao, T. A. Smith, G. G. Qiao, and X. Zhang, "Surfactant-mediated formation of polymeric microlenses from interfacial microdroplets", Soft Matter 957-964 (2014).

[203] A. Ma, J. Xu, and H. Xu, "Impact of spontaneously adsorbed hydroxide ions on emulsification via solvent shifting", The Journal of Physical Chemistry C 118, 23175-23180 (2014).

[204] J. Aubry, F. Ganachaud, J.-P. C. Addad, and B. Cabane, "Nanoprecipitation of Polymethylmethacrylate by Solvent Shifting: 1. Boundaries", Langmuir 25, 1970-1979 (2009).

[205] E. Lepeltier, C. Bourgaux, and P. Couvreur, "Nanoprecipitation and the "ouzo effect": Application to drug delivery devices", Advanced Drug Delivery Reviews 71, 86 - 97 (2014).

[206] R. Stepanyan, J. Lebouille, J. Slot, R. Tuinier, and M. C. Stuart, "Controlled nanoparticle formation by diffusion limited coalescence", Physical Review Letters 109, 138301 (2012).

[207] M. Graužinytė, J. Forth, K. A. Rumble, and P. S. Clegg, "Particlestabilized water droplets that sprout millimeter-scale tubes", Angewandte Chemie International Edition 54, 1456-1460 (2015).

[208] A. D. Stroock, S. K. W. Dertinger, A. Ajdari, I. Mezi, H. A. Stone, and G. M. Whitesides, "Chaotic mixer for microchannels", Science 295, 647-651 (2002).

[209] T. T. Al-Housseiny, P. A. Tsai, and H. A. Stone, "Control of interfacial instabilities using flow geometry", Nature Physics 8, 747-750 (2012).

[210] F. Haudin, J. H. E. Cartwright, F. Brau, and A. De Wit, "Spiral precipitation patterns in confined chemical gardens", Proceedings of the National Academy of Sciences 111, 17363-17367 (2014).

[211] O. Steinbock, "Complexity from precipitation reactions", Proceedings of the National Academy of Sciences 111, 17346-17347 (2014).

[212] X. H. Zhang and W. Ducker, "Interfacial oil droplets", Langmuirr 24, 110-115 (2008).

[213] S. Schubert, J. T. Delaney, Jr, and U. S. Schubert, "Nanoprecipitation and nanoformulation of polymers: from history to powerful possibilities 
beyond poly(lactic acid)", Soft Matter 7, 1581-1588 (2011).

[214] Y. Xibo, D. Marco, F. Amy, A. Pierre, G. S. G., F. Etienne, K. J. L., G. François, and B. Julien, "Simple but precise engineering of functional nanocapsules through nanoprecipitation", Angewandte Chemie International Edition 53, 6910-6913 (2014).

[215] H. Lhuissier, D. Lohse, and X. Zhang, "Spatial organization of surface nanobubbles and its implications in their formation process", Soft Matter 10, 942-946 (2014).

[216] H. Schlichting and K. Gersten, Boundary layer theory, 8th edition (Springer Verlag, Berlin) (2000).

[217] S. Grossmann and D. Lohse, "Fluctuations in turbulent Rayleigh-Bénard convection: The role of plumes", Physics of Fluids 16, 4462-4472 (2004).

[218] E. Lajeunesse, J. Martin, N. Rakotomalala, and D. Salin, "3d instability of miscible displacements in a hele-shaw cell", Physical Review Letters 79, 5254-5257 (1997).

[219] I. Bischofberger, R. Ramachandran, and S. R. Nagel, "Fingering versus stability in the limit of zero interfacial tension", Nature Communications 5, 5265 (2014).

[220] T. E. Faber, Fluid dynamics for physicists (Cambridge University Press) (1995).

[221] V. N. Prakash, Y. Tagawa, E. Calzavarini, J. M. Mercado, F. Toschi, D. Lohse, and C. Sun, "How gravity and size affect the acceleration statistics of bubbles in turbulence", New Journal of Physics 14, 105017 (2012).

[222] N. Ishida, T. Inoue, M. Miyahara, and K. Higashitani, "Nano bubbles on a hydrophobic surface in water observed by tapping-mode atomic force microscopy", Langmuir 16, 6377-6380 (2000).

[223] D. Lohse and X. Zhang, "Pinning and gas oversaturation imply stable single surface nanobubble", Physical Review E 91, 031003(R) (2015).

[224] S. R. German, X. Wu, H. An, V. S. J. Craig, T. L. Mega, and X. Zhang, "Interfacial nanobubbles are leaky: Permeability of the gas/water interface", ACS Nano 8, 6193-6201 (2014).

[225] R. Wang, L. Cong, and M. Kido, "Evaluation of the wettability of metal surfaces by micro-pure water by means of atomic force microscopy", Applied Surface Science 191, 74-84 (2002).

[226] X. H. Zhang, N. Maeda, and V. S. J. Craig, "Physical properties of nanobubbles on hydrophobic surface in water and aqueous solutions", Langmuir 22, 5025-5035 (2006).

[227] X. H. Zhang, A. Quinn, and W. A. Ducker, "Nanobubbles at the interface between water and a hydrophobic solid", Langmuir 24, 4756-4764 
(2008).

[228] A. C. Simonsen, P. L. Hansen, and B. Klösgen, "Nanobubbles give evidence of incomplete wetting at a hydrophobic interface", Journal of Colloid and Interface Science 273, 291-299 (2004).

[229] M. H. Uddin, S. Y. Tan, and R. R. Dagastine, "Novel characterization of microdrops and microbubbles in emulsions and foams using atomic force microscopy", Langmuir 27, 2536-2544 (2011).

[230] X. Wang, B. Zhao, W. Ma, Y. Wang, X. Gao, R. Tai, X. Zhou, and L. Zhang, "Interfacial nanobubbles on atomically flat substrates with different hydrophobicities", ChemPhysChem 16, 1003-1007 (2015).

[231] L. Zhang, C. Wang, R. Tai, J. Hu, and H. Fang, "The morphology and stability of nanoscopic gas states at water/solid interfaces", ChemPhysChem 13, 2188-2195 (2012).

[232] C. Xu, S. Peng, G. O. Qiao, V. Gutowski, D. Lohse, and X. Zhang, "Nanobubbles on a warmer substrate", Soft Matter 10, 7857-7864 (2014).

[233] S. Peng, D. Lohse, and X. Zhang, "Spontaneous pattern formation of surface nanodroplets from competitive growth", ACS Nano 9, 1191611923 (2015).

[234] J. Yang, J. Duan, D. Fornasiero, and J. Ralston, "Very small bubble formation at the solid-water interface", The Journal of Physical Chemistry B 107, 6139-6147 (2003).

[235] B. M. Borkent, S. de Beer, F. Mugele, and D. Lohse, "On the shape of surface nanobubbles", Langmuir 26, 260-268 (2010).

[236] B. Song, W. Walczyk, and H. Schönherr, "Contact angles of surface nanobubbles on mixed self-assembled monolayers with systematically varied macroscopic wettability by atomic force microscopy", Langmuir 27, 8223-8232 (2011).

[237] R. Garcia and R. Perez, "Dynamic atomic force microscopy methods", Surface science reports 47, 197-301 (2002).

[238] A. Chengara, A. D. Nikolov, D. T. Wasan, A. Trokhymchuk, and D. Henderson, "Spreading of nanofluids driven by the structural disjoining pressure gradient", Journal of Colloid and Interface Science 280, 192 - 201 (2004).

[239] D. N. Sibley, A. Nold, N. Savva, and S. Kalliadasis, "A comparison of slip, disjoining pressure, and interface formation models for contact line motion through asymptotic analysis of thin two-dimensional droplet spreading”, Journal of Engineering Mathematics 94, 19-41 (2014).

[240] S. Peng, I. Devic, H. Tan, D. Lohse, and X. Zhang, "How a surface nanodroplet sits on the rim of a microcap", Langmuir 32, 5744-5754 
(2016).

[241] V. Mathai, V. N. Prakash, J. Brons, C. Sun, and D. Lohse, "Wakedriven dynamics of finite-sized buoyant spheres in turbulence", Physical Review Letters 115, 124501 (2015).

[242] D. H. Ballard, "Generalizing the hough transform to detect arbitrary shapes", Pattern Recognition 13, 111-122 (1981).

[243] M. J. E. Najafabadi and H. Pourghassem, "A novel method for improving edge detection using negative and gamma correction functions", in Intelligent Computation and Bio-Medical Instrumentation (ICBMI), 2011 International Conference on, 60-63 (IEEE) (2011).

[244] J. M. Stauber, S. K. Wilson, B. R. Duffy, and K. Sefiane, "On the lifetimes of evaporating droplets", Journal of Fluid Mechanics 744, R2 (2014). 



\section{Summary}

The evaporation and dissolution of droplets in the multi-component system are omnipresent in nature, science, and many advanced technologies. In the droplets, the two-way interactions between the fluid properties and hydrodynamics make the dynamical behaviors complicated. To have a quantitative understanding of these processes is not only a challenge but also significantly crucial for many applications, such as inkjet printing, liquid-liquid extraction, DNA mapping, emulsion formation, and so on. In this thesis, we studied the evaporation and dissolution of droplets in ternary systems with the "ouzo effect" through a combined experimental-numerical-theoretical approach.

In Part [1, we explored the evaporating and dissolving processes of "ouzo" (water, ethanol, and anise oil) droplets on surfaces. Through a series of studies, we revealed microdroplet nucleation processes triggered by the evaporation or dissolution of the droplets in ternary systems and consequently induced dynamical behaviors of the droplets. The local diffusion rates of different components determines the variation of the local composition and the consequent preference of the microdroplet nucleation: at the contact line of the evaporating flat "ouzo" droplet $(\S 2)$, at the top of the evaporating spherical "ouzo" droplet $(\S 3$, and at the middle of the dissolving spherical "ouzo" droplet (§4). To gain insight into the evaporation process of sessile mixture droplets, we numerically and experimentally performed a systematic investigation in $\S 5$, by successively increasing the mixture complexity from pure water over a binary water-ethanol mixture to the ternary "ouzo" mixture. We provided an in-depth discussion on several important factors to the hydrodynamics inside the evaporating droplets, including preferential evaporation, evaporative cooling effect, thermal and solutal Marangoni flows, and their interplays as well. Based on these understandings, we proposed a generalized diffusion model in $\S 3$ to predict the evaporative mass loss rate of the evaporating spherical ouzo droplets. To explore the multi-diffusion process at the interface of the dissolving multicomponent droplets, in $\S 4$ we proposed a one-dimensional model by integrating multi-diffusion process theory and the liquid-liquid equilibrium theory. Besides, there are many interesting phenomena discovered along with the investigation in Part [ including self-formed oil ring at the droplet con- 
tact line $(\S 2$, wrapping of nucleated oil $(\S 3)$, suspending microdroplets rings (§4). In this part, we fully revealed the complicated dynamical behaviors in the evaporating and dissolving multicomponent droplets.

In Part [II, we performed exploratory research on the application of the evaporating multicomponent droplets. Inspired by the interesting phenomenon observed in $\S 2$, we employed the self-formed oil ring in evaporation-driven particle fabrication to prevent the formation of the pinning contact line in $\S 6$. That is, the evaporating colloid ouzo droplets acquire a "self-lubrication" ability. Through our method, the evaporation-driven particles assembly is practicable by using the commonly used hydrophobic surfaces and the fabrication of high-porosity supraparticles with controllable shapes is feasible by changing the initial ratio of oil to nanoparticles in the colloidal drops. The appearance of self-formed lubricating oil layer conduces to the complete detachment of the generated supraparticles, which allows the repeated utilization of the surfaces. We believe that our approach of particle synthesis with many advantages including, scalability, flexibility, operability, and cost-efficiency, and chemical-consumption-efficiency, will give tremendous contributions to the mass production of the advanced particles.

In the last Part [II], our attention focused on the nucleated nanodroplets on the surface induced by the "ouzo effect." We used the solvent exchange method to form the surface nanodroplets in a narrow channel with controlled flow conditions. In $\S 7$, we used this method to form surface nanodroplets in different controlled conditions and statistically analyzed the volume of the nucleated nanodroplets. Based on the idea that the growths of surface nanodroplets result from the exposure of the droplet nuclei to an oil oversaturation pulse during the exchange process, we developed a theoretical framework and revealed a $3 / 4$ power law relationship between the volume of the nanodroplets and the Péclet number of the flow. The convection effects by density difference during the exchange process were also discussed. In $\S 8$, we have developed a comprehensive three-dimensional (3D) spherical cap fitting procedure for the accurate extraction of the morphologic characteristics of complete or truncated spherical caps from atomic force microscopy (AFM) images. Our method integrates several advanced digital image analysis techniques to construct a 3D spherical cap model. We expect the developed 3D spherical-cap fitting procedure will find many applications in imaging analysis. 


\section{Samenvatting}

Het verdampen en oplossen van druppels in multi-compomenten systemen zijn alomtegenwoordig in natuur, wetenschap en vele geavanceerde technologieën. De tweewegsinteractie tussen vloeistofeigenschappen en hydrodynamica maakt het dynamisch gedrag van druppels gecompliceerd. Een kwantitatief begrip van deze processen krijgen is niet alleen uitdagend maar ook cruciaal voor veel toepassingen, zoals inktjet printen, vloeistof-vloeistof extractie, DNA mapping, emulsie formatie, en zo verder. In deze thesis hebben we de verdamping en oplossing van druppels in ternaire systemem met het "ouzo effect" bestudeerd met van een gecombineerde experimentele-numerieke-theoretische aanpak.

In deel I, hebben we de verdamping en oplossing processen van "ouzo" (water, ethanol en anijsolie) druppels op oppervlakken verkent. Door een serie van studies hebben we microdroppel nucleatie processen onthuld die getriggerd worden door de verdamping of oplossing van de druppels in ternaire systemen en de daardoor geïnduceerde dynamische gedragingen van de druppels. De lokale diffusiesnelheden van verschillende componenten bepaalt de variatie van de lokale compositie en de bijgevolge voorkeur van microdruppel nucleatie: bij de contactlijn van de platte verdampende "ouzo" druppel $(\S, 2)$, bij de top van de verdampende sferische "ouzo" druppel ( $\S 3)$, en in het midden van de oplossende sferische "ouzo" druppel (§4). Om inzicht te krijgen in het verdampingsproces van sessiele druppels van mengsels hebben we een systematisch numeriek en experimenteel onderzoek gedaan in $\S 5$, door het opvoeren van de mengselcomplexiteit van puur water via een binair water-ethanol mengsel naar het ternaire "ouzo" mengsel. We hebben verschillende belangrijke factoren voor de hydrodynamica in de verdampende druppels diepgaand bediscussieerd, inclusief preferentiële verdamping, verdampingskoeling effect, thermische en oplossings Marangoni stromingen, en de interactie tussen deze factoren. Gebaseerd op deze begrippen, hebben we een gegeneraliseerd diffusie model voorgesteld in $\S, 3$ om de verdampingsmassaverliessnelheid van verdampende sferische ouzo druppels te voorspellen. Om de multi-diffusie processen op het oppervlak van oplossende multicomponent druppels te verkennen, in $\S 4$ hebben we een ééndimensionaal model voorgesteld, dat de multi-diffusie pro- 
cestheorie en de vloeistof-vloeistof evenwichtstheorie integreert. Daarnaast, er zijn veel interessante fenomenen ontdekt tijdens het onderzoek in deel I, onder andere zelf-geformeerde oliering bij de druppel contactlijn $(\S, 2)$, inpakken van genucleërde olie $(\S, 3)$, hangende microdruppel ringen $(\S, 4)$. In dit deel hebben we de ingewikkelde dynamische gedragingen in verdampende en oplossende multicomponent druppels volledig onthuld. In deel II hebben we verkennend onderzoek gedaan naar de toepassing van verdampende multicomponent druppels. Geïnspireerd door de interessante fenomenen geobserveerd in $\S 2$, hebben we de zelfvormende oiliering in verdampingsgedreven deeltjesproductie gebruikt om de formatie van een gepinde contactlijn te voorkomen in $\S 6$. Dat wil zeggen, de verdampende colloïde ouzo druppels verkrijgen een "zelfsmerend" vermogen. Door middel van onze methode is verdampingsgedreven deeltjes assemblage uitvoerbaar door algemeen gebruikte hydrofobe oppervlaken te gebruiken en de productie van hoog-poreuze supradeeltjes met te controleren vormen is mogelijk door de initiële ratio van olie en nanodeeltjes te veranderen in de colloïde droppels. Het verschijnen van zelf-vormende smerende olielaag leidt tot de complete ontkoppeling van de gegenereerde supradeeltjes, wat het hergebruik van de oppervlakken mogelijk maakt. Wij geloven dat onze aanpak van deeltjessynthese met vele voordelen zoals, schaalbaarheid, flexibiliteit, operabiliteit, en kosten-efficiëntie, een geweldige bijdrage zal leveren aan de massaproductie van geavanceerde deeltjes.

In het laatste deelIII, richtte onze aandacht zich op de genucleërde nanodruppels op het oppervlak, geïnduceerd door het "ouzo effect." We gebruikten de oplossing uitwisseling methode om oppervlakte nanodruppels te formeren in een smal kanaal met gecontroleerde stromingscondities. In $\S, 7$, gebruikten we deze methode om oppervlakte nanodruppels in verschillende gecontroleerde condities te maken en analyseerden statistisch het volume van de genucleërde nanodruppels. Gebaseerd op het idee dat de groei van oppervlakte nanodruppels een gevolg is van de blootstelling van druppel nucleatiepunten aan een olie oververzadigde puls gedurende het uitwisselingsproces, hebben we een theoretisch kader ontwikkeld en een $3 / 4$ powerlaw relatie tussen het volume van de nanodruppels en het Péclet nummer van de stroming onthuld. De convectie effecten bij dichtheidsverschillen gedurende het uitwisselingsproces zijn ook bediscussieerd. In $\S 8$, hebben we een veelomvattend driedimensionaal (3D) bolkap fitting procedure ontwikkeld om de morfologische karakteristieken van complete of afgeknotte bolkappen accuraat af te leiden uit atoomkrachtmicroscopie (AFM) afbeeldingen. Onze methode integreert verschillende geavanceerde digitale beeldanalyse technieken om een 3D bolkap model te construeren. Wij verwachten dat de ontwikkelde 3D bolkap fitprocedure veel toepassingen zal vinden in beeldanalyse. 


\section{Scientific output}

\section{Publications}

- H. Tan, Y.X. Huang, J. Meng, Hilbert statistics of vorticity scaling in two-dimensional turbulence, Physics of Fluids, 26(1), 015106 (2014).

- X. Zhang, Z. Lu, H. Tan, L. Bao, Y. He, C. Sun, D. Lohse, Formation of surface nanodroplets under controlled flow conditions, PNAS, 112, 9253-9257 (2015). (Chapter 7)

- S. Peng, I. Devic, H. Tan, D. Lohse, X. Zhang, How a surface nanodroplet sits on the rim of a microcap, Langmuir, 32, 5744-5754 (2016).

- H. Tan, S. Peng, C. Sun, X. Zhang, D. Lohse, 3D spherical-cap fitting procedure for (truncated) sessile nano- and microdroplets \& -bubbles,

EPJE, 39, 106 (2016). (Chapter 8)

- H. Tan, C. Diddens, P. Lv, J. Kuerten, X. Zhang, D. Lohse, Evaporation-triggered microdroplet nucleation and the four life phases of an evaporating ouzo drop, PNAS, 113, 8642-8647 (2016). (Chapter 2)

- C. Diddens, H. Tan, P. Lv, M. Versluis, J. Kuerten, X. Zhang, D. Lohse, Evaporating pure, binary and ternary droplets: thermal effects and axial symmetry breaking,

J. Fluid Mech., 823, 470-497 (2017). (Chapter 5)

- H. Tan, C. Diddens, M. Versluis, H. Butt, D. Lohse, X. Zhang, Self-wrapping of an ouzo drop induced by evaporation on a superamphiphobic surface, Soft Matter, 13, 2749 (2017). (Chapter 3)

- H. Tan, S. Wooh, H. Butt, X. Zhang, D. Lohse, Porous supraparticle assembly through self-lubricating evaporating colloidal ouzo drops,

Submitted. (Chapter 6) 
- H. Tan, A. Mohammed, C. Diddens, J. Li, M. Versluis, X. Zhang, D. Lohse, Microdroplets nucleation by dissolution of a multicomponent drop in a host liquid, Submitted. (Chapter 4)

- L. Mandemaker, M. Filez, G. Delen, H. Tan, X. Zhang, D. Lohse, and B. M. Weckhuysen,

Time-resolved in-situ liquid-phase atomic force microscopy and infrared nanospectroscopy on the formation of metal-organic framework thin-films, J. Phys. Chem. Lett., 9, 1838 (2018).

- Y. Li, P. Lv, C. Diddens, H. Tan, H. Wijshoff, M. Versluis, D. Lohse, Evaporation-triggered segregation of sessile binary droplets, Phys. Rev. Lett., 120 (22), 224501 (2018).

- X. Li, Y. Wang, B. Zeng, Y. Li, H. Tan, H. Zandvliet, X. Zhang, D. Lohse, Entrapment and dissolution of microbubbles inside microwells, Submitted.

- H. Tan, C. Diddens, M. Versluis, X. Zhang, D. Lohse, Oscillation of a dissolving multicomponent drop in a host liquid, In preparation.

\section{Teaching experience}

- Student supervision, UT

Ali Akash Mohammed, internship student from Paris Diderot University (April - July 2017) Junyi Li, internship student from University of Science and Technology of China (July - Sep 2017)

- Teaching assistant, UT

Experimental techniques in Physics of Fluids (graduate course) 2017

Course coordinator: Prof. Alvaro Marin

Physics of Fluids (undergraduate course) 2017

Course coordinator: Prof. Michel Versluis and Prof. Devaraj van der Meer

Experimental techniques in Physics of Fluids (graduate course) 2015

Course coordinator: Prof. Chao Sun 


\section{Invited and selected talks}

- 24th Dutch Soft Matter Meeting (Invited), Leiden, Netherlands (June 2018)

- American Physical Society, 70th Annual Meeting - DFD, Denver, USA (Nov. 2017)

- MESA+ Meeting 2017 (Invited), Enschede, Netherlands (Sep. 2017)

- Droplets 2017, UCLA, Los Angeles, USA (July 2017)

- Burgers Symposium 2017 (Invited), Lunteren, Netherlands (May 2017)

- Physics@Veldhoven 2017, Veldhoven, Netherlands (Jan. 2017)

- American Physical Society, 69th Annual Meeting - DFD, Portland, USA (Nov. 2016)

\section{Scientific workshops \& Schools}

- 8th Complex Motion in Fluids (1 week), University of Cambridge, UK (Sep. 2017)

- 7th Complex Motion in Fluids (1 week), University of Twente, Netherlands (June 2016)

- JM Burgerscentrum courses (national research school for fluid mechanics), Netherlands $(2015,2016,2017)$ 



\section{Acknowledgements}

Time flies! It is already five and half years ago since the first time I came to Physics of Fluids Group (PoF) for my three-month Master internship. I appreciate Prof. Quan Zhou, Prof. Chao Sun, and Assoc. Prof. Yongxiang Huang (Master supervisor) for offering this opportunity, which starts my story with PoF. During the internship, I had an enjoyable time in Twente Water Tunnel under the daily supervision of Dr. Vivek Prakash. It is natural for me to decide to come back for my Ph.D. study.

Dear Detlef, thank you for giving me the opportunities to carry on research in PoF, for providing continuous support throughout my Ph.D. pursuits. In the past four years, you not only taught me how to perform scientific research but also trained me to have own sense of smell on science. Numerous comments, suggestions, and questions from you continually pushed me to challenge myself to go higher. Your joy and enthusiasm for science are contagious and motivate me all the time. Your help and advice on my long-term scientific development make me feel humbled and very grateful. I also respect your ability to lead such a big group and your capability to teach students following their aptitudes. I am proud of being one of your Ph.D. students.

Dear Xuehua, thank you for guiding me towards the engineering chemistry field and providing valuable supports from chemistry views. I benefited from your critical comments, which are challenging and push me to learn more knowledge on chemistry. Considering that chemistry is not my fond course since middle school, this process is a breakthrough for me. Without you, it would have been impossible for me to finish this thesis.

Dear Michel, thank you for introducing to me many fantastic experimental techniques and providing helpful suggestions from an experimental point of view. The improvement of my experimental skills benefits from you. Your patience in our talk encouraged me to ask different kinds of scientific questions, and then you shared your experience with me, for which I am thankful too.

Dear Chao, thank you for arising my interests in the experiments of fluids physics, and for providing advice and help throughout my Ph.D. career. The enjoyable working experience with you during the internship was one of the reasons I decided to come back. During my Ph.D. study, you helped me many 
times when I was in trouble, which I appreciate from my heart.

I would like to gratefully acknowledge my collaborators for their assistance and contributions to this Ph.D. thesis. Dear Christian, it is a privilege to start our collaborations at the very early stage of my $\mathrm{Ph} . \mathrm{D}$. study, giving credit to Detlef and Hans. I benefit from numerous in-depth discussions with you. I learned a lot by working with you, particularly in writing and editing manuscripts. It made me feel warm when you offered help to correct my thesis. Dear Pengyu, thank you for teaching me the confocal microscope technique and sharing with me some lab skills. Dear Sanghyuk, thank you for offering critical suggestions from materials science views and introducing materials science knowledge. Many thanks to Prof. J. G. M. Kuerten, Prof. Hans-Jürgen Butt, who provided many important discussions and valuable contributions to my Ph.D. thesis research.

My grateful acknowledgment also goes to my students for their assistance and contributions to this Ph.D. thesis. Dear Ali, thank you for choosing me as your internship supervisor, and for your hard working during the internship. Your study enthusiasm is the best encouragements for my first supervision experience. I appreciate a lot of fun with you on the basketball court and batavierenrace 2017. Dear Junyi, thank you for being my second internship student, and for your hard working during your stay. It is enjoyable to learn something about your favorite street dance.

It is a pleasure to pay tribute to Joanita, Bas, Gert-Wim, Martin B., and Dennis $G$. for all of their supports these years. Your technical input and assistance improve the efficiency of work in the group. Joanita, thank you for taking care of the administrative work and many help to make my stay comfortable. Bas, thank you for helping me order different materials for my experiments, even though my requests usually came as urgent. I also would like to extend my tribute to the nano-lab technicians Mark and Henke for the supports on scanning electron microscope.

Dear Ivan, Mathijs, and Anupam, I am very grateful that four of us from four different countries, in the same autumn, stepped into this office, and then have this four-year Ph.D. studying journey together. Ivan, indeed, you are the reason behind the lively atmosphere of our office. You shared many interesting things with me, in particular, the old photo with your rock-style hair, which I like honestly. There is a lot of fun with you in our lovely basketball court. It is my pleasure that we have a collaboration work in this journey. Mathijs, thank you for sharing much interesting dutch news and information. The sounds by your different "toys" have become a contribution to the lively atmosphere of our office. The chess game with you in our office-outing drinking was enjoyable. I thank you for helping me translate the summary to Dutch. Anupam, you 
are the guy with equations in our office. I like your insights on the scientific discussions of our office. Unfortunately, it is still too hard to reach a single conclusion from our office discussion. I appreciate having a lot of fun with the three of you. I will miss you and look forward to our reunion many years later.

I would like to extend my gratitude to more people. To all the members of the committee, thank you for spending your time to come to Enschede for my defense. To Prof. Andrea Prosperetti, Prof. Jacco Snoeijer, Assi. Prof. Alvaro M., and Prof Yuliang Wang, thank you for the helpful discussions and excellent courses. To my current and previous labmates, Martin S, Mazi, Myethre, Richard, and Jiaming, thank you for the help and for together creating a pleasant working atmosphere in lab ME 208. To our Monday meeting group members, Richard, José, Mikhail, Pallav, Steven, Yanshen, Xiaolai, thank you for the inspiring discussions. To Claas, my labmates, my internship roommates, and my friend, thank you for providing help during my internship, for giving many valuable and critical suggestions both on science and life to me in the past five years. To Corentin, Michiel L, Alexander K, Varghese, Guillaume, Jun Luo, Tim, thank you for many detailed discussion on experimental techniques. To my colleagues and friends, Alexander B., Arjan, Álvaro S., Biljana, Carola, Dennis B., Luoqin, Maaike, Marie-Jean, Nakul, Peter, Pieter, Pim, Rianne, Rodolfo, Rodrigo, Ruben, Sander H, Sander W, Shuai, Utkarsh, Vamsi, Xiumei, Yujie, Zhenhua, thanks for the numerous office fun, laboratory fun, (outside) lunches, coffee corner discussions, summer school and conference experiences, basketball events, running trainings, batavierenrace, film-making projects, spontaneous parties, now and then, here and there.

Outside the PoF group, I am very grateful to have some family friends here with whom we have shared so much over the years, and in our language. Lovely little Hehe, Xiaojue, Qiulu, lovely little Isa, Lin Luo, Yue Li, Ji Lian, Yaxing, Xin Chen, Yantao, Yibo, Zhengchao, Jia Liang, Songchuan, Qixiu, Mengqi, Haitao, Pengu, Lijie, thanks for sharing so much fun with my family during the years. Making dumplings and cooking together in Chinese festivals, talking about family life and kids, playing Chinese card games after dinner, all of these are enjoyable and unforgettable memories to my family.

Last but not least, I would like to appreciate many Dutch people (doctors, drivers, midwives) who provides the big help to my family in past four years. All your help have made our family feel warm, and we are very grateful to you and this country.

Finally, I own my most profound love and gratitude to my family:

爸爸妈妈, 感谢您们的养育之恩和这些年支持。如今已为人父的我更加体 
会到了父爱母爱的伟大与厚重。您们仍然是我心底最最深处的那份依靠, 我 永远爱您们! 老婆, 从相识相爱到如今有了我们自己的温馨家庭和可爱的孩 子, 我们已经一起走过了十几年。感谢你对我和这个家的默默付出, 感谢有 你陪伴一起走过的每个日夜。我永远爱你! 谭博文, 你的出现给我们的生活 带来了无限的乐趣与幸福。你是我们一生中最美丽的礼物。爸爸妈妈永远爱 你!

Enschede, Summer 2018 